\title{
Channel Measurement and Modeling in Complex Environments
}

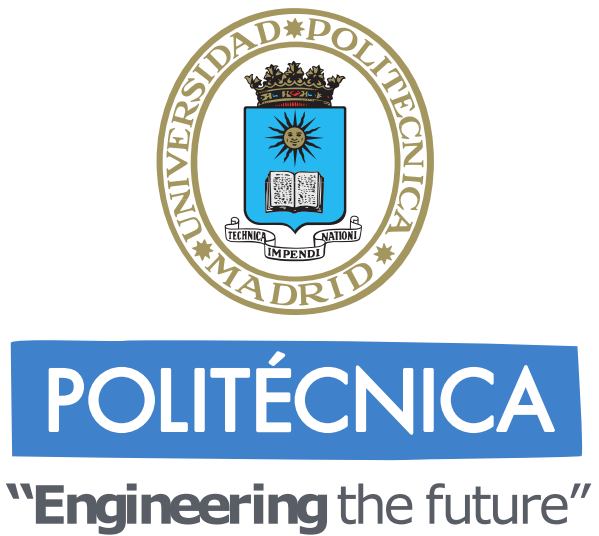

Lei Zhang

School of Telecommunications Systems and Engineering

Technical University of Madrid

ETSIST-UPM

A thesis submitted for the degree of

Doctor of Philosophy

Madrid, September 2016 
Doctorado en Ingeniería de Sistemas y Servicios para la Sociedad de la Información

\begin{tabular}{|c|c|c|}
\hline \multicolumn{3}{|c|}{ Tesis Doctoral } \\
\hline Título & \multicolumn{2}{|c|}{ Channel Measurement and Modeling in Complex Environments } \\
\hline Autor & \multicolumn{2}{|c|}{ Lei Zhang } \\
\hline Director & Dr. César Briso Rodríguez & $V^{\circ} B^{\circ}$ \\
\hline \multicolumn{3}{|c|}{ Tribunal } \\
\hline Presidente & \multicolumn{2}{|c|}{ Dr. José Ignacio Alonso Montes } \\
\hline Vocal & \multicolumn{2}{|c|}{ Dr. Luis Castedo Ribas } \\
\hline Vocal & \multicolumn{2}{|c|}{ Dr. Ke Guan } \\
\hline Vocal & \multicolumn{2}{|c|}{ Dr. Miguel Ángel Gómez Laso } \\
\hline Secretario & \multicolumn{2}{|c|}{ Dr. Juan Moreno García-Loygorri } \\
\hline \multicolumn{3}{|l|}{ Suplente } \\
\hline \multicolumn{3}{|l|}{ Suplente } \\
\hline $\begin{array}{c}\text { Lugar y } \\
\text { fecha de lectura }\end{array}$ & \multicolumn{2}{|c|}{$\begin{array}{l}\text { E.T.S.I y Sistemas de Telecomunicación } \\
\text { de Septiembre de } 2016\end{array}$} \\
\hline Calificación & & \\
\hline
\end{tabular}

El Presidente

La secretaria

Los vocales

Tesis Doctoral para la obtención del título de Doctor por la Universidad Politécnica de Madrid 
To my beloved family. 


\section{Acknowledgements}

This dissertation is not only a milestone of my life but also the result of many experiences I have encountered in Madrid from dozens of remarkable individuals who I also wish to acknowledge.

First and foremost, I would like to express my deepest gratitude to my supervisor, Prof. César Briso Rodríguez, who offered his continuous advice and encouragement throughout my Ph.D. study. I have no way to repay the systematic guidance and great effort he put into training me in the scientific field.

A very special thanks to Prof. David W. Matolak, who gave me lots of valuable suggestions during my research stay at the University of South Carolina. I really admired his meticulous attitude on research and enjoyed every discussion with him. Many thanks to our partners in the cooperation institutes (BJTU, UDC, TONGJI): Prof. Ke Guan, Qi Wang, Prof. José A. García-Naya, José Rodríguez-Piñeiro, and Xuesong Cai. Thanks to my colleagues in GRC: Dr. Jean Raphaël Olivier Fernandez, Sergio Pérez Jiménez, David Alonso Sáez, etc. It would have been a dreary journey without my folks in Campus Sur: Daniel Gómez Lobo Garca, Dr. Yuanjiang Huang, Shengjing Sun, Yongjun Pan, Yu Chen, Xin Li, etc.

Last, but certainly not least, I would like to give my most sincere thanks to my parents, for their many years of selfless love and patience support. Thanks to my wife Qin and our soon-to-be-born baby, who have given me boundless strength and great courage. I love you beyond words.

Lei Zhang

Madrid, July 2016 


\begin{abstract}
The fast development of new technologies such as high-speed rail (HSR) with high reliability, safety and capacity promote the evolution process of corresponding dedicated wireless communication system from narrowband to broadband. The main challenges for the communication system design and network planning in complex environments include: the enclosed space in underground systems; the rapidly time-varying channel in high mobility scenarios, a variety of complex structures in composite scenarios, such as stations, tunnels, cuttings, viaducts, etc.; and the extra losses caused by the vehicle's structure. Thus, Wireless propagation characteristics in complex scenarios are of importance for the design and implement reliable and efficient communications in modern communication systems.

This dissertation analyzes the key challenges for wireless channel characterization in the complex environments. Then a series of propagation measurements conducted in real complex scenarios are presented as the test cases. For the underground system, two typical types of curved tunnels are firstly investigated by the mean of narrowband measurements. The statistical characterization of the path loss exponent, shadow fading distribution, autocorrelation, and cross-correlation are parametrized and compared with different configurations. Furthermore, wideband measurement campaigns conducted in a modern subway system at $980 \mathrm{MHz}$ and $2450 \mathrm{MHz}$ are described in detail. The time dispersion is analyzed with the power delay profile in different regions. The principal parameters, such as mean power and root mean square (RMS) delay are extracted to establish region-based tapped delay line (TDL) models. Moreover, the electromagnetic reverberation in underground system is characterized by the reverberation time, absorption coefficient, and quality factor (Q) as the functions of distance for the first time. Also, the transition distance between station and tunnel is modeled based on the $\mathrm{Q}$ versus distance.
\end{abstract}


For high mobility scenarios, a series of wideband measurements carried out in an HSR composite scenario are described. The PDPs are also analyzed to generate the TDL channel models in different regions. Then the corresponding 3D ray-tracing simulation is employed to achieve a deterministic channel model for validating the measurement and also providing the frequency dispersion. The small-scale fading characteristics and the effect of Doppler shift are statistically analyzed. Propagation mechanism inside the mass transit system is another "hot" topic. The study of the outdoor-to-indoor and indoor-to-indoor radio propagation characteristics inside trains is also conducted in this dissertation. Based on the measurements of actual LTE coverage receiving by external and internal antennas, the extra loss caused by the train's physical structure is estimated. Also, the propagation measurements inside the high-speed train (HST) are presented. Results show the waveguiding effects inside the HST carriage. The log-distance path loss models are parameterized, and small-scale fading statistics for various propagation links are proposed and compared. The extensive analysis and discussions made in this dissertation are expected to reflect the propagation mechanism behind the observations in complex environments. The quantitative results and channel models are useful to realize the high-performance wireless communication system in complex scenarios. 


\section{Resumen}

El rápido desarrollo de la alta velocidad ferroviaria (HSR) con una alta fiabilidad, seguridad y capacidad está demandando actualmente la evolución de los sistemas de telecomunicaciones inalámbricas empleados en este entorno hacia sistemas banda ancha y alta capacidad. Por ello, las comunicaciones con los trenes de alta velocidad son uno de los principales casos de estudio para el desarrollo y diseño de sistemas de comunicaciones en entornos complejos. Este entorno incluye: espacios cerrados en los sistemas sub-terraneos; canal rápidamente variable en el tiempo en los escenarios de alta movilidad, variedad de estructuras complejas en escenarios compuestos como estaciones, túneles, trincheras, viaductos, etc., y las pérdidas adicionales causadas por la estructura del vehículo. Por lo tanto, el modelado de las características de propagación en estos escenarios es de gran importancia para el diseño e implementación de sistemas de comunicaciones fiables y requeridas en los sistemas de comunicación modernos.

Esta tesis analiza los principales retos para la caracterización de la propagación en entornos complejos principalmente de ferrocarriles de alta velocidad y metropolitanos. Para ello se han realizado multitud de medidas de propagación realizados en escenarios complejos reales, las cuales se han analizado y empleado para el modelado del canal. En los ferrocarriles metropolitanos se han estudiado en detalle distintos tipos de túneles curvos. El estudio se realiza primero analizando el comportamiento en banda estrecha, la caracterización estadística del exponente de perdidas de propagación, la distribución estadística y sus parámetros de autocorrelación y de correlación cruzada. Estos parámetros se comparan con diferentes configuraciones de antenas y en diferentes entornos. Por otra parte, se ha realizado también un modelado de la propagación en banda ancha basado en la realización de varias campañas de medida realizadas por medio de un sistema de sonda de canal empleado en entornos de metro a $980 \mathrm{MHz}$ y 
2.450 MHz. De estas medidas se obtiene el perfil y retardo de potencia en diferentes regiones. Los principales parámetros, como la potencia media y retardo (RMS) se extraen para establecer modelos de línea de retardo con tomas basada en regiones (TDL). Otro aspecto novedoso es la aplicación de la teoría de la reverberación electromagnética en radiocomunicaciones en entornos cerrados. En este caso se aplica a los sistema ferroviarios subterráneos donde el entorno se caracteriza en primer lugar con el tiempo de reverberación, coeficiente de absorción, y el factor de calidad (Q) . Ademas,esta teoría se ha aplicado también a la distancia de transición entre la estación y el túnel, modelando en base a el factor de calidad Q frente a la distancia. Para los escenarios de alta movilidad, se describen una serie de mediciones de banda ancha realizadas en un escenario compuesto HSR. Los resultados son empleados para modelar la propagación en diferentes regiones. Estos resultados se han aplicado a un modelo de simulación de trazado de rayos 3D para lograr un modelo de canal determinista para la validación de la medidas y proporcionando también la dispersión de frecuencia. Las características de desvanecimiento de pequeña escala y el efecto del desplazamiento Doppler, el cual se analizan estadísticamente. Otros novedosos puntos de estudio son el mecanismo de propagación en el interior de los sistema de transporte público y el estudio de la propagación vehículo a vehículo entre trenes. Para ello se realizan medidas de la cobertura de sistemas 4G LTE real la recepción por antenas externas e internas, la perdida adicional causada por la estructura física del tren se estima. Ademas, se presenta la medición de propagación en el interior del tren de alta velocidad (TAV). los resultados muestran los efectos de gua de ondas dentro del carro HST. el camino de la distancia de registro modelos de perdida son parametrizados, y se proponen y se comparan las estadísticas de desvanecimiento de pequeña escala para varios enlaces de propagación. Se espera que el extenso análisis y debates realizados en esta tesis para modelar el mecanismo de propagación dentro de las observaciones en entornos complejos. Los resultados cuantitativos y modelos de canal son útiles para realizar el sistema de comunicación inalámbrica de alto rendimiento en escenarios complejos principalmente túneles y grandes recintos cerrados. 


\section{Contents}

Acknowledgement

Abstract

Resumen vi

Contents viii

List of Figures $\quad$ xi

$\begin{array}{lll}\text { List of Tables } & \text { Xv }\end{array}$

1 Introduction 1

1.1 Motivation . . . . . . . . . . . . . . . . 2

1.2 Contributions . . . . . . . . . . . . . . . 5

1.3 Outline of thesis . . . . . . . . . . . . 7

2 Radio Propagation and Channel Modeling 9

2.1 Propagation Mechanism . . . . . . . . . . . . . . . 10

2.1.1 Path Loss and Fading . . . . . . . . . . . . . . . 11

2.1.2 Fading Classification ... . . . . . . . . . . . 13

2.2 Channel Modeling . . . . . . . . . . . . . . . . . . . . . . 16

2.2.1 Large-scale fading Models . . . . . . . . . . . . . . . . 18

2.2.2 Amplitude Statistics . . . . . . . . . . . . . . . . 21

2.2.3 Time Dispersion . . . . . . . . . . . . . . . 24

2.2.4 Tapped Delay Line Model . . . . . . . . . . . . . . . . . 30 
2.2.5 Angular Spread . . . . . . . . . . . . . . . . . 32

2.2.6 Doppler Spread . . . . . . . . . . . . . . . . 33

2.2.7 Deterministic Modeling: Ray Tracing . . . . . . . . . . . . 34

2.3 Discussion . . . . . . . . . . . . . . . . . . . . 36

3 Channel Measurement Systems 38

3.1 Channel Sounders . . . . . . . . . . . . . . . . . . . . . . 40

3.2 Channel Sounder Development . . . . . . . . . . . . . . 44

3.2.1 Requirements . . . . . . . . . . . . . . . . . 44

3.2.2 Self-developed Channel Sounders . . . . . . . . . . . . 46

3.2.3 SDR-based Channel Sounder . . . . . . . . . . . . . . . 52

3.2.4 Multi-antenna system . . . . . . . . . . . . . . 55

3.3 Discussions . . . . . . . . . . . . . . . . . . . 57

4 Propagation in Underground Environments $\quad 59$

4.1 Part I: Narrowband Measurements in Curved Tunnels . . . . . . . 61

4.1.1 Measurement Scenarios and Configurations . . . . . . . . . 62

4.1.2 Measurement Results Analysis . . . . . . . . . . . . . . . . 64

4.1.3 Large-Scale Fading Characteristics . . . . . . . . . . . . . 69

4.2 Part II: Broadband Measurements in Station and Tunnel . . . . . 77

4.2.1 Measurements Scenarios and Configurations . . . . . . . . 78

4.2.2 Measurement Results Analysis . . . . . . . . . . . . . . . . 81

4.2.3 Characterization and Modeling . . . . . . . . . . . . . 84

4.3 Discussion . . . . . . . . . . . . . . . . . 100

5 Propagation in High Mobility Scenarios 103

5.1 Hardware Systems . . . . . . . . . . . . . . . . . . 106

5.2 Case I: HSR Station . . . . . . . . . . . . . . . . . . 107

5.2.1 Measurement Scenarios and Configurations . . . . . . . . . 107

5.2.2 Measurement Results Analysis . . . . . . . . . . . . . . . . 109

5.3 Case II: HSR Composite Scenario . . . . . . . . . . . . . . . . . . 111

5.3.1 Measurement Scenarios and Configurations . . . . . . . . . 111

5.3.2 Measurement Results Analysis . . . . . . . . . . . . . . . . 113

5.3.3 Time Dispersion Analysis . . . . . . . . . . . . . . 116 
5.3.4 3D Ray Tracing . . . . . . . . . . . . . . . . . 117

5.4 Discussion . . . . . . . . . . . . . . . . . . . . . . . . . . 124

6 Propagation inside Mass Transit Systems 127

6.1 Layer I: Outdoor-to-Indoor Coverage . . . . . . . . . . . . . . . . 131

6.1.1 Measurement Scenarios and Configurations . . . . . . . . . 131

6.1.2 Results Analysis . . . . . . . . . . . . . . . . . 135

6.2 Layer II: Propagation inside Trains . . . . . . . . . . . . . . . . . 138

6.2.1 Measurement Scenarios and Configurations . . . . . . . . . 138

6.2.2 Result Analysis \& Channel Characterization . . . . . . . . 140

6.3 Discussion . . . . . . . . . . . . . . . . . . . . . 151

7 Conclusion \& Future Work 153

7.1 Conclusion . . . . . . . . . . . . . . . . . . 153

7.2 Future Work . . . . . . . . . . . . . . . . . . 155

$\begin{array}{ll}\text { References } & 158\end{array}$

$\begin{array}{ll}\text { Appendix: Antenna Patterns } & 185\end{array}$ 


\section{List of Figures}

1.1 Wireless communication systems and complex environments in ITS, via: etsi.org. . . . . . . . . . . . . . . . . . . 3

2.1 Multipath propagation scenario. . . . . . . . . . . . . . 11

2.2 Path loss, large-scale fading, and small-scale fading. . . . . . . . . 13

2.3 Fading classification. . . . . . . . . . . . . . . . . . . 14

2.4 Log distance path loss model. . . . . . . . . . . . . . . . . . 19

2.5 The delay spread conception, via: www.sharetechnote.com. . . . . 25

2.6 The SalehValenzuela model. . . . . . . . . . . . . . . . . . . 26

2.7 Tapped delay line model. . . . . . . . . . . . . . . . . . 31

2.8 A ray tracing example: (a) propagation scenario: UPM Campus Sur, Carretera de Valencia, km. 7, Madrid, Spain; (b) ray tracing illustration; (c) comparison with the measurement results. . . . . 35

2.9 Time and frequency dispersion. . . . . . . . . . . . . . 36

2.10 Flow chart of the channel model development. . . . . . . . . . . 37

3.1 Principle of a periodic pulse sounder. . . . . . . . . . . . . . . 42

3.2 Principle of a correlative channel sounder. . . . . . . . . . . . . . 44

3.3 High-ended channel sounder transmitter front-end. . . . . . . . . 46

3.4 elf-developed channel sounder receiver front-end. . . . . . . . . . . 47

3.5 Photograph of the channel sounder system. . . . . . . . . . . . . 48

3.6 Single pulse synchronization. . . . . . . . . . . . . . . . . . . . . 49

3.7 Block diagram of the portable channel sounder. . . . . . . . . . 51

3.8 Protograph of the portable channel sounder . . . . . . . . . . 52

3.9 Photograph of the hexagon multi-antenna system. . . . . . . . . 56 
3.10 Channel sounding with multi-antenna system. . . . . . . . . 56

4.1 Measurement setup and photographs in the arched and semicircular tunnels. . . . . . . . . . . . . . . . . . . 63

4.2 Measured received power (small-scale fading removed). Comparison with different polarizations in the traditional curved arched tunnel with $500 \mathrm{~m}$ RoC at: (a) $920 \mathrm{MHz}$, (b) $2400 \mathrm{MHz}$, and (c) $5705 \mathrm{MHz}$; Comparison with different radii of curvature in semicircular tunnels with horizontal polarization at (d) $920 \mathrm{MHz}$, (e) $2400 \mathrm{MHz}$, and (f) $5705 \mathrm{MHz}$. . . . . . . . . . . . 65

4.3 Path loss and probability density of shadow fading in the arched tunnel at $5705 \mathrm{MHz}$ with horizontal polarization. . . . . . . . . 67

4.4 Empirical autocorrelation coefficient of shadow fading fitting in the theoretical models by LS regression in the arched tunnel at 2400 $\mathrm{MHz}$ with horizontal polarization; $d_{c o r}(0.5)$ and $d_{c o r}\left(e^{-1}\right)$ represent the $d_{c o r}$ equal to 0.5 and $e^{-1}$, respectively. . . . . . . . . . . . 68

4.5 Diagrammatic sketch and photo of the measurement scenarios and setup of the 1 st Test. . . . . . . . . . . . . . . . . . 78

4.6 Diagrammatic sketch and photos of the measurement scenarios and setup of the 2 nd Test. . . . . . . . . . . . . . . . . . . 79

4.7 Simplified diagram of the first group testing. . . . . . . . . . . . . 79

4.8 Simplified diagram of the second group testing. . . . . . . . . . . 80

4.9 Empirical PDP samples in the subway station at $2450 \mathrm{MHz}$, when the train stopped at different positions. . . . . . . . . . . . . . 82

4.10 Empirical PDP samples in the subway station at $980 \mathrm{MHz}$, when the train stopped at different positions. . . . . . . . . . . . . 83

4.11 PDPs variation versus distance at $980 \mathrm{MHz}$. . . . . . . . . . . . 84

4.12 PDPs variation versus distance at $2450 \mathrm{MHz}$. . . . . . . . . . . . 85

4.13 The reverberation phenomenon for qualifying the acoustic environment, via: karagioza.com. . . . . . . . . . . . . . 86

4.14 The concept of delay power spectrum in reverberation model. . . 87

4.15 Definition of PDP's slope. . . . . . . . . . . . . . . . . . . . . 91

4.16 RMS delay of TEST II at $980 \mathrm{MHz}$ and $2450 \mathrm{MHz}$. . . . . . . . 93 
4.17 Region division for evaluating the different influences from the different zone in tunnel along track: (a) $2450 \mathrm{MHz}$; (b) $980 \mathrm{MHz}$. . 94

4.18 Q-factor variations and fittings versus distance at $980 \mathrm{MHz}$ and 2450 MHz. . . . . . . . . . . . . . . . . . . . . . . . . . . . . 98

4.19 Transition model base on the Q-factor variations at $980 \mathrm{MHz}$ and $2450 \mathrm{MHz}$. . . . . . . . . . . . . . . . . . . . . . . 99

5.1 Measurement scenarios and configurations in the HSR station. . . 108

5.2 The PDPs in LOS and NLOS regions at two frequencies. . . . . . 109

5.3 The received power versus time delay and test points at two frequencies. . . . . . . . . . . . . . . . . . . 110

5.4 The measurements in the HSR composite scenario: (a) sketch of the composite scenario; (b) photo of the test site; (c) aerial view of the HSR composite scenario from google earth; (d) photo of the tunnel portal. . . . . . . . . . . . . . . . . . . . . 112

5.5 Representative PDPs in different regions:(a) tunnel portal; (b) cutting 1; (c) the part of viaduct close to cutting 1 (Viaduct 1); (d) middle part of viaduct (Viaduct 2); (e) the part of viaduct close to cutting 2 (Viaduct 3); (f) cutting 2. . . . . . . . . . . 113

5.6 PDPs along the cutting and viaduct in the composite region at 950 $\mathrm{MHz}$ and $2150 \mathrm{MHz} \ldots \ldots$. . . . . . . . . . . . . . . 115

5.7 RMS delay spread at two frequencies . . . . . . . . . . . 116

5.8 Scenario model of the HSR composite scenario and a snapshot of ray tracing process. . . . . . . . . . . . . . . . . . . . . . . 118

5.9 Comparisons of the PDPs between the measurement and the proposed deterministic model at two frequencies . . . . . . . . . . . 121

5.10 PDP along scenario time at different frequencies . . . . . . . . . . 122

5.11 Small-scale fading along the scenario time at two frequencies. . . . 123

5.12 Doppler power spectrum along scenario time at two frequencies. . 124

5.13 Mean Doppler shift and RMS Doppler spread along scenario time at two frequencies. . . . . . . . . . . . . . . . . 125

6.1 Various wireless communication links onboard the train. . . . . . 130 
6.2 Outdoor test environments: (a) LTE BTS tower structure with the eNodeB antennas at $2.6 \mathrm{GHz}$ mounted at a height of $20 \mathrm{~m}$; (b) snapshot of the test train by Pablo Nieto (via: www.ferropedia.es);

(c) four external antennas on the roof of the train carriage. . . . . 132

6.3 Measurement site: (a) measurement site from Google map; (b)BTS with eNodeB 2.6 GHz located at Antequera station. Simulation of the LTE radio coverage along the test track. . . . . . . . . . . . . 133

6.4 Estimated SNR values by means of measurements corresponding to both BTS-Train and BTS-Mobile links. . . . . . . . . . . . 136

6.5 Estimated SNR difference between the B2T and B2M links. . . . 137

6.6 Indoor test environments: (a) interior of the commercial train car (via: www.talgo.com); (b) cross section of the test train. . . . . . 139

6.7 Photographs and Setups of the testbed transmitter and receiver. . 140

6.8 Received signal power level of Station-Mobile link at 2.4 GHz and 5.7 GHz. . . . . . . . . . . . . . . . . . . . . . . . 141

6.9 Received signal power level of the Intra-Carriage and Inter-Carriage links inside the HST. . . . . . . . . . . . . . . . . . . . . . 143

6.10 Received signal power level of the Intra-Carriage and Inter-Carriage links the metro train. . . . . . . . . . . . . . . . . . . . . . . 144

6.11 Path loss and least-square fittings for Intra-Carriage and InterCarriage links inside HST and Metro train. . . . . . . . . . . . . . 145

6.12 Path loss and least-square fittings for Station-Mobile links inside HST . . . . . . . . . . . . . . . . . . . 146

6.13 Nakagami distribution fitting with small-scale amplitude fading statistics of wireless links. . . . . . . . . . . . . . . . . . . . 149

6.14 Measurement setup for testing the influence of the AoA. . . . . . 150

6.15 Normalized path loss versus AoA. . . . . . . . . . . . . . . . 150

1 The photo of the HG2414P flat panel antennas and the radiation patterns at two frequencies. . . . . . . . . . . . . . 185

2 The photo of the R\&S HL025 log-periodic antenna and the radiation patterns at two frequencies. . . . . . . . . . . . . . 186

3 Antennas applied in the measurements in HSR, via: www.L-com.com.187 


\section{List of Tables}

2.1 Three cases for the cluster and multipath resolution. . . . . . . . 27

3.1 High-end channel sounder profiles. . . . . . . . . . . . . 50

3.2 Portable channel sounder profiles . . . . . . . . . . . . . . 52

4.1 Datasheet of the test systems for the narrowband measurements in subway tunnels. . . . . . . . . . . . . . . . . . 62

4.2 PLE and the standard deviations of shadow fading in the curved arched tunnel . . . . . . . . . . . . . . . . . . . . . . 69

4.3 PLE and the standard deviations of shadow fading in the curved semicircular tunnel . . . . . . . . . . . . . . . . . . . . 70

4.4 PLEs and standard deviations of shadow fading distribution among tunnels and various propagation traffic scenarios . . . . . . .

4.5 Comparison of large-scale fading characteristics between curved arched tunnel scenario and standard scenarios in WINNER . . . 74

4.6 Decorrelation distance statistic in the curved arched and semicircular tunnel . . . . . . . . . . . . . . . . . . . . 75

4.7 Statistic values of the ME, Std., and RMSE between measurements and the exponential model and the 802.16J model . . . . . . . . 76

4.8 Cross-correlation of the shadow fading between the $920 \mathrm{MHz}, 2400$ $\mathrm{MHz}$, and $5705 \mathrm{MHz}$ in curved subway tunnels . . . . . . . . 77

4.9 Channel sounder configurations. . . . . . . . . . . . . . . . . . . 81

4.10 Train's influences on the mean power, RMS delay and reverberation time in the subway station. . . . . . . . . . . . . . . 92

4.11 Key parameters for TDL models in different regions. . . . . . . . 95 
4.12 Key parameters for reverberation models in different regions. . . . 97

5.1 Channel sounder setups _. . . . . . . . . . . . . . 106

5.2 Antenna informations . . . . . . . . . . . . . . . . 107

5.3 Parameters of time dispersion at two frequencies . . . . . . . . . . 110

5.4 Statistical results for modeling the time dispersion at two frequencies 117

5.5 Analysis of normalized short-term fading . . . . . . . . . . . . . 123

6.1 LTE testbed configurations. . . . . . . . . . . . . . . . . 134

6.2 Parameters of the testbed transmitter. . . . . . . . . . . . . . . . 140

6.3 Comparison for path loss model factors in Intra-Carriage, InterCarriage, Station-Mobileand from the literature. . . . . . . . . . . 147

6.4 Akaike weights of four distribution candidates, and the fitted shape factors of Nakagami and Weibull distribution . . . . . . . . . . . . 147

6.5 Shape factors and K-factors for small-scale characterizations. . . . 148

6.6 PLE characterization as a function of the AoA . . . . . . . . . . . 151 


\section{Chapter 1}

\section{Introduction}

Since the famous assertion:

"The fundamental problem of communication is that of reproducing at one point, either exactly or approximately, a message selected at another point."

from Claude E. Shannon's stunning paper "A Mathematical Theory of Communication" is published in 1948, communication has been qualitatively and quantitatively modeled as a statistical process underlying information theory. It brings the practical result of Shannon-Hartley law for the channel capacity of a Gaussian channel and inspires the further research on the channel capacity of a noisy channel, including the promise of perfect loss-free communication [1].

As the name suggests, propagation channel refers to the structure or path along which the signal is propagated, such as wire pair, coaxial cable, waveguide, optical fiber, or radio path. For a wired transmission system, the signal is delivered in fixed transmission channel with stable propagation characteristics. Whereas the propagation path of a radio signal is uncertainty. The technology developing also bring many challenges as limited bandwidth, limited power and processing data in the dynamic environment. The transmitted signal with limited power suffers propagation loss along the distance due to the energy dissipation. In the meantime, radio propagation is vulnerable to the propagation environment. The reflection, diffraction, and scattering phenomenon in propagation environment impact the signal and lead to the time and frequency dispersions at the 
receiver side. The effects of multipath propagation include constructive and destructive interference and phase shifting of the signal result in the increasing of bit error rate and decreasing of channel capacity. Hence, theoretical analysis and channel modeling on the wireless propagation are always the hot topics and difficulties in wireless communication system design and development. This thesis is dedicated to the study of channel measurement and modeling in various complex environments.

\subsection{Motivation}

Wireless communication has undergone a rapid development in the past decades. After the $1 \mathrm{G}$ (first generation) mobile communication system released in the $1980 \mathrm{~s}$, and $2 \mathrm{G}$ (second generation) proposed in the 1990s, a large number of commercial communication systems, such as GSM (Global System for Mobile Communications), 3G (Third Generation), Wi-Fi (Wireless Fidelity), WiMAX (World Interoperability for Microwave Access), and 4G LTE (Fourth Generation Long Term Evolution), are gradually proposed and deployed in the past two decades. From the roots of analog based $1 \mathrm{G}$ networks to todays truly broadband-ready $4 \mathrm{G}$ technology in extensive deployment, the wireless communication industry paves the way for the worldwide mobile broadband access, and promises the great innovations in the future. Although the wireless communication systems have played an indispensable role in our daily lives, the communication technology itself is still continually upgrading. For instance, the requirements and KPI (Key Performance Indicators) of $5 \mathrm{G}$ have been established. The $5 \mathrm{G}$ is the fifth generation mobile communication technology as the extension of $4 \mathrm{G}$. The application scenarios of $5 \mathrm{G}$ are divided into two major categories: mobile Internet, and IoT (Internet of Things). Therefore, aside from Mobile Internet, $5 \mathrm{G}$ is expected to solve the requirement of massive machine wireless communication, thus, significantly promote the development of Internet of Vehicles and Industry of Internet.

The information and communication technologies (ICT) are widely implied in the road, railway, water, and air transport systems, as well as the navigation systems as illustrated in Fig. 1.1. The Intelligent Transport Systems (ITS) include telematics and all types of communication techniques for different appli- 
cation links, such as In-Vehicle (IN-V) communications, Vehicle-to-Vehicle (V2V) communications between adjacent vehicles, Vehicle-to-Infrastructure (V2I), and Vehicle-to-Cloud (V2C). In general, the various types of ITS communication system rely on specialized radio services and communication technologies.

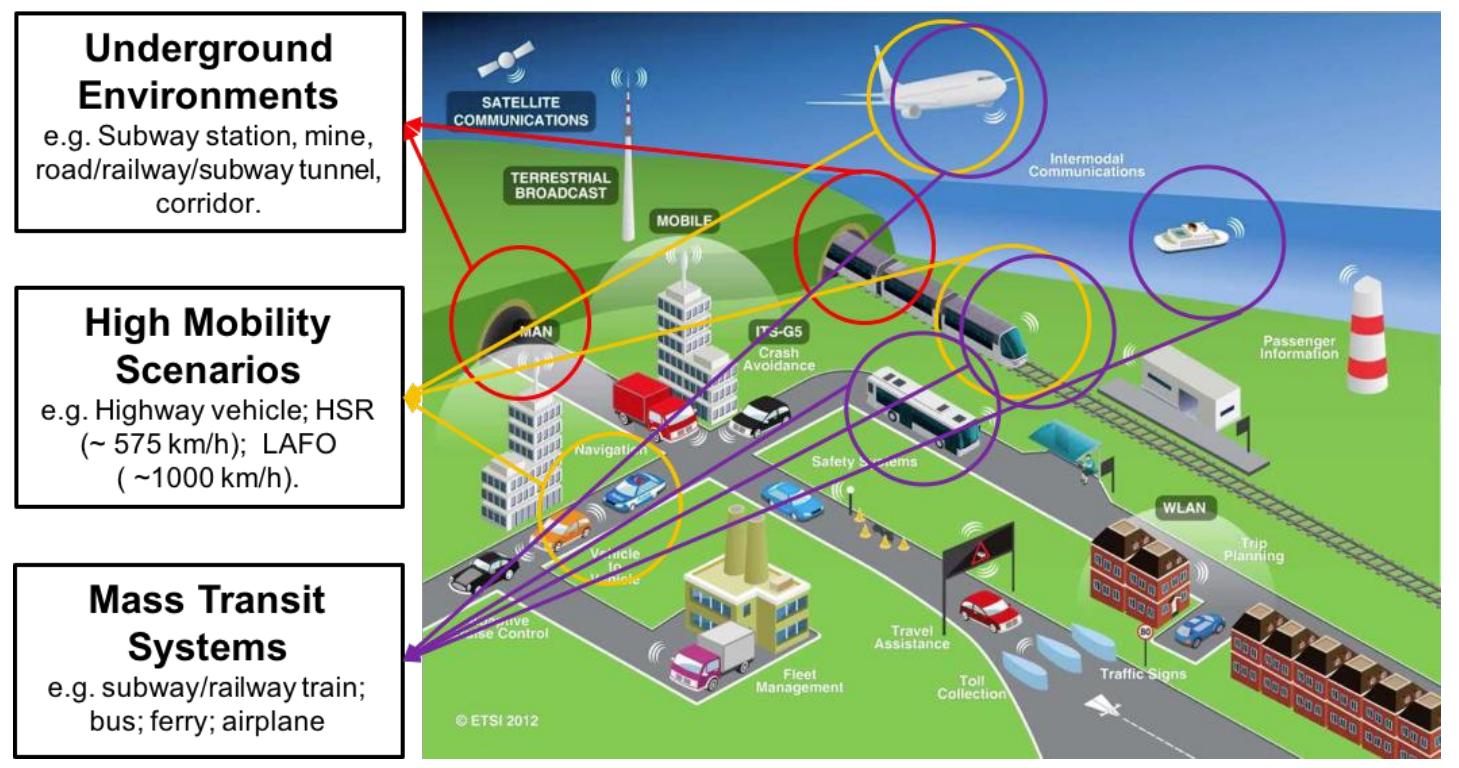

Figure 1.1. Wireless communication systems and complex environments in ITS, via: etsi.org.

The propagation channel is a factor that cannot be neglected for communication system design and deployment. A detailed understanding of the propagation channel is a mandatory premise of the communication system deployments in particular scenarios. A channel model represents the knowledge of how the propagation environment impairs and distorts the radio signals, in other words, what do the radio signal experienced in the propagation channel [2]. Thus, an accurate channel model contributes to the designpredicting, and comparison the performance of the new wireless communication systems in actual conditions. However, complex environments often mean rapidly changing terrains, irregular spatial structures, the richness of absorption surfaces, or unavoidable obstacles. Radio propagation in these complex environments usually faces particular challenges such as great attenuation, severe fading, or serious interface. These significant problems motivate the studies on the propagation in complex environments to achieve the dedicated channel models for characterizing the propagation channel. 
Then, the methods for mitigation the impairments and distortions on the wireless signals can be devised and evaluated based on the dedicated channel models. Eventually, the adverse factors of the reliable and efficient data transmission can be mitigated or eliminated in system design and network planning.

In Fig. 1.1, three representative complex environments have been highlighted. Channel measurement and modeling in these complex environments face additional challenges, due to:

- Underground environments: e.g., mine system, road tunnel, railway tunnel, subway tunnel, subway station, etc. These systems are built in the underground environments with confined spaces, and surrounding by the solid, rocks, or concretes. The confined space leads to unique propagation mechanisms, such as waveguiding effect, dense scatterers, time delay, energy storage, etc. These effects vary with the volumes of spaces, physical properties of the surfaces, and transformation of spatial structures.

- High Mobility scenarios: e.g., high-speed vehicle, high-speed railway (HSR), low altitude flying object (LAFO), etc. The speedy carriers or objects in these scenarios bring the time varying propagation channel in rapidly changed terrains. Therefore, the wireless communication system in high mobility scenarios always suffers failures of frequent handover and varying channel dispersions across different propagation regions. In the meantime, the difficult terrains in various regions are mutually affected the radio propagation.

- Mass Transit systems: e.g., railway/subway train, bus, ferry, airplane, etc. wireless communication systems for these mass transit systems are not only required to be responsible for the adequacy, stability, and safety of control signal transmission, but also expected to provide broadband network access for passenger's convenience and satisfaction. Nevertheless, the carriage structures of these vehicles can significantly impair the wireless propagation links for outdoor-to-indoor and indoor-to-indoor the vehicles. Hence, the evaluation of radio coverage for onboard mobile user and radio propagation inside the mass transit systems are of importance. 
In short, these three types of complex environments have their unique features that can affect the wireless channel and result in exceptional propagation characteristics. Therefore, the customized channel measurement and channel modeling based on the geometric information are worthy fields of study.

Notably, rail transport system including High-speed railway (HSR), intercity railway, light rail and subway as the key enabling factor for sustainable mobility have offered great simplicity for people's traveling and low-cost logistics. In the meantime, rail transport as an environment-friendly transport mode with less energy consumption and limited air pollution than other modes underpins economic growth. In Europe, the EU Transportation White Paper(European Commission 2011) has targeted a more sustainable, efficient, and competitive transport service that will shift from road to rail in the future [3]. Hence, channel measurement and modeling in complex railway environments can be representative. Propagation in railway environment is particularly complex: i) various geometric structures, such as the interior of the train, subway stations, tunnels, viaducts, cuttings, semi-closed stations, etc. ii) multiple communication standards coexisting. For instance, GSM, GSM-R, LTE, and TETRA work at $900 \mathrm{MHz}$ frequency band. At 2.4 GHz frequency band, there are Wi-Fi, UMTS (Universal Mobile Telecommunications System), LTE, CBTC, and pending LTE-R. Moreover, there are many wireless radio systems deployed for driverless underground systems work at 5.8 $\mathrm{GHz}$ [4]. iii) different kinds of reflection or scattering surface with various materials, such as steel, concrete, etc. These factors lead to the existing channel models can not match the propagation scenarios in railway. Therefore, this thesis adopts the channel measurement and modeling in complex railway environments as the exemplary cases for further analysis and investigations.

\subsection{Contributions}

The main contributions of this thesis can be summarized as follows:

1. The widely-used channel measurement systems are reviewed. Based on the periodic pulse sounding technique, two self-developed channel sounders are designed and deployed for both narrowband and wideband propagation measurements. Also, the promising software-defined radio (SDR) applied as new channel 
sounder is introduced and initially tested.

2. The existing studies on the issues of the radio propagation measurement and channel modeling in underground environments, high mobility scenarios, and mass transit systems are surveyed.

3. The first-hand practical measurement results are obtained and presented in this thesis, including the narrowband measurements in subway curved tunnels with various configurations; the wideband measurements in subway station and tunnel at $980 \mathrm{MHz}$ and $2150 \mathrm{MHz}$; the wideband measurements in HSR station and a composite regions contain tunnel, cutting, and viaduct; the radio coverage for outdoor and indoor high-speed train; propagation loss inside the trains.

4. By the mean of extensive measurements, the dedicated channel models are proposed for each complex environments. The log-distance path loss models are established in curved tunnels and inside trains with path loss exponents and shadowing fading standard deviations. These key parameters are compared and with other standard scenarios for further analysis. For wideband measurements, the time dispersions are characterized and compared with delay spread in different regions of the specific environments. Based on the RMS delay values, the regionbased Tapped Delay Line (TDL) models in subway and railway are presented and analyzed.

5. The decorrelation distance and cross-correlation of the shadow fading are investigated in the curved tunnels. Besides, the electromagnetic reverberation theory is firstly introduced in subway system for channel modeling. The absorption coefficients calculated by the Sabin's and Eyring's formulas are presented in different regions inside a subway tunnel. Also, the Q factor models based on the reverberation times and carrier frequencies can be helpful for defining the transition distances from the subway station into the tunnel with different configurations. Also, the ray-tracing approach is employed for evaluating the frequency dispersion in HSR composite scenarios. The Doppler spread at two frequencies in different regions are obtained and compared.

6. The measurement results and corresponding channel models can be helpful for radio frequency network planning and wireless communication system updating in both subway and railway. Besides, the extending study in other complex environments can reference the relative work in this thesis. 


\subsection{Outline of thesis}

The rest of this dissertation is organized as follows:

Chapter 2 introduces the background of the fundamental radio propagation theories and classical channel models. The state-of-the-art review of the researches in radio propagation measurements and modeling is also conducted in this chapter. This includes the detailed descriptions of the classification of fading in time and frequency domains; the widely used models in for large-scale and small-scale fading, such as log-distance path loss model, small-scale fading distributions, TDL model, and deterministic modeling.

Chapter 3 surveys the widely-used channel sounding techniques for time domain and frequency domain measurements, including the periodic pulse-based, the sliding correlation-based, sweeping frequency-based approaches. Also, the self-developed periodic pulse based channel sounder testbeds adopted in the following measurements are described in detail. Besides, the software defined radio (SDR)-based channel sounder and multi-antenna system are introduced for further testing.

Chapter 4 presents the channel measurement and modeling in underground systems. First, the narrowband channel sounding in the curved subway tunnel is described, the large-scale characterization and modeling based on the corresponding results are analyzed and compared with straight tunnel and other standard scenarios. Second, the influences on the radio propagation from the subway station and tunnel are evaluated individually by the wideband measurements. The measurements configurations and scenarios are carefully presented. The key factors, such as delay spread and reverberation time are extracted and parameterized. Finally, the region-based TDL models and Q factors along the distance in the subway station and tunnel are established and analyzed in this chapter.

Chapter 5 demonstrates the channel measurement and modeling in high mobility scenarios. The propagation measurements in HSR station and an HSR composite regions at $950 \mathrm{MHz}$ and $2150 \mathrm{MHz}$ are emphasized as the test cases. The time dispersion in the HSR station in LOS and NLOS scenarios are analyzed and characterized in Case I. The HSR composite scenario in Case II includes tunnel portal, cutting, and viaduct. The channel characterizations in these com- 
posite regions are separately analyzed and parameterized. The key factors such as RMS delay and the number of channel taps are calculated for forming regionbased TDL models. Furthermore, a 3D ray-tracing simulation is conducted and validated. The stochastic analysis and frequency dispersion are presented based on the simulation results.

Chapter 6 focuses on the propagation inside the mass transit systems. The LTE radio coverage testing by the mean of the power receiving by the external antenna and internal antenna. Moreover, the propagation losses inside the highspeed train and metro train are carefully described and separated into two links: Intra-Carriage and Inter-Carriage links. The large-scale and small-scale fading are characterized and compared within the similar scenarios such as airplane cabins and corridors. Besides, the propagation from the station to the mobile inside the train carriage at different frequencies and angle of arrivals are also presented.

Eventually, the obtained results are summarized as the conclusion of the thesis, and the topics for further research are also addressed in Chapter 7. 


\section{Chapter 2}

\section{Radio Propagation and Channel Modeling}

Scientists have been tantalized by the research on electromagnetism since James Clerk Maxwell (1831 - 1879) first formulated his famous four equations. His theory of electromagnetism has discovered that changing electric field induced a magnetic field and also vice-versa, and demonstrated that electricity, magnetism, and light are all manifestations of the same phenomenon: the electromagnetic (EM) field. Then, the electromagnetic wave has proved mathematically and confirmed by the German physicist Heinrich Hertz in 1886. His experiments not only detect the existence of electromagnetic waves but also verify that they propagate at the speed of light.

It is well known that electromagnetic wave propagation depends on the properties of the transmission medium in which they travel. The propagation medium or electromagnetic path connecting the transmitter and receiver is defined to the communication channel [5]. For the wireless communication channel, it used to work in the UHF $(0.3-3 \mathrm{GHz})$, and SHF $(3-30 \mathrm{GHz})$ frequency bands, and fundamentally dedicate to deliver sufficient signal power to the receiver at the end of the radio link to achieve some performance objective.

Knowledge of the propagation behavior of a radio channel is of vital importance to understand and design for a radio communication system. The properties of the propagation channel govern the level and quality of the received signal. 
Chapter 2. Radio Propagation and Channel Modeling

Moreover, like any other form of EM energy, reflection, refraction and diffraction may occur within the radio propagation. Thus the received signal may be a constructive or destructive combination of several signals that have traveled by different paths with different time delays, which lead to the distorting of the resultant signal. Unquestionably, propagation characteristics give insight into wireless communication system performance.

The background knowledge, such as the propagation manifestation and classification are introduced in this chapter. Also, the underlying principle of multipath propagation and widely accepted theory involved in the following chapters are explained in this chapter.

\subsection{Propagation Mechanism}

The wireless signal, like light waves and all other forms of EM radiation, typically travel in a direct path in the ideal free space, where usually infers that the signal attenuation versus distance. The so-called free space assumes the propagation environment between the transmitter and receiver is empty, and neither absorbing obstacles nor reflecting surfaces are considered to affect radio frequency energy. The atmosphere in this region is perfectly uniform and non-absorbing medium. Furthermore, the influence of the earth surface is assumed to be entirely absent. Since the separation $d$ between the transmitter and receiver is much larger than the far field distance $d_{f}\left(d_{f}=\frac{2 D^{2}}{\lambda}, \lambda\right.$ : wavelength; $D$ : the largest dimension of the antenna), the EM waves act like the plane waves that dominate all other components in the far field, i.e. the radiating antenna can be considered as a point source with negligible physical dimensions. The far field region is also termed as Fraunhofer region; then the Friis equation holds in this region to derive the free space path loss (FSPL) at distance $d$ as follows [6]:

$$
F S P L(d)=\frac{P_{t} G_{t} G_{r}}{P_{r}}=\frac{P_{t} G_{t} G_{r} \lambda^{2}}{(4 \pi)^{2} d^{2} L}
$$

where $P_{r}$ is the received power, $P_{t}$ is the transmitted power, $G_{r}$ is the receiver antenna gain, $G_{t}$ is the transmitter antenna gain, $d$ is the separation distance between transmitter and receiver in meters, $L$ is the system loss factor not re- 
lated to the propagation. $\lambda$ is the wavelength in meters. As Eq. 2.1 shows, the attenuation of RF energy between the transmitter and receiver behaves according to an inverse-square law.

\subsubsection{Path Loss and Fading}

However, when the signal travels through the wireless channel in the actual propagation environment, the presence of trees, people, buildings, mountains, and other obstructions result in the signal undergoing many kinds of propagation effects as shown in Fig. 2.1. Three of these physical effects are primary considered: reflection, diffraction, and scattering.

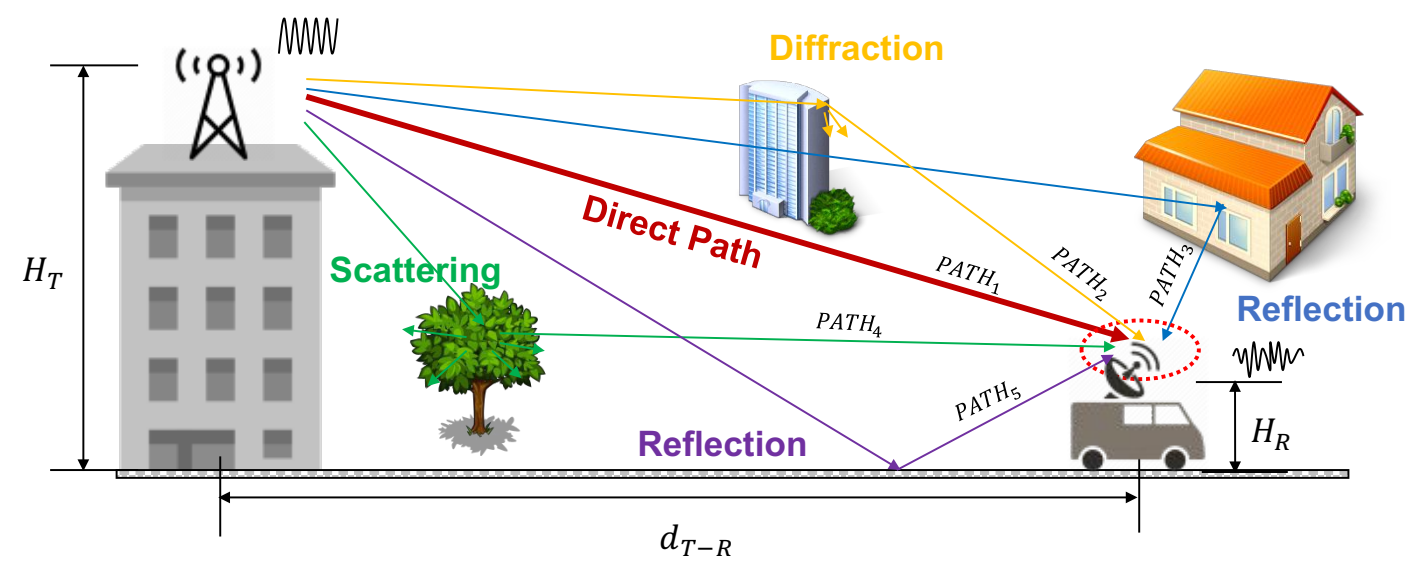

Figure 2.1. Multipath propagation scenario.

- Reflection occurs when the radio waves impinge on a smooth surface of the object which has much larger dimensions than the wavelength of the incident wave. In practice, the incident energy may be reflected partly or entirely, which is depends on the material properties, wave polarization, the angle of incidence, and the carrier frequency. Reflections could occur from the surface of the earth, the buildings, or major contributed by the walls, floors, ceilings, and items of furniture for indoor environments.

- Diffraction is a phenomenon occurring when the wave interacts with an obstacle that having sharp irregularities (edges, wedges, etc.). The radio 


\section{Chapter 2. Radio Propagation and Channel Modeling}

waves can propagate behind the obstruction with decreased field strength, which is the vector sum of the electric field components of all the secondary wavelets [6]. Diffraction is also known as shadowing.

- The scattering provides extra energy at the receiver, which is caused by scattering energy in all directions from the trees, the rough surfaces, small objects, or irregularities. Scattering occurs when the medium through the wave is traveling contains objects which are much smaller than the wavelength of the radio wave. Comparatively speaking, scattering is most difficult to predict [7].

The multipath propagation is defined as the effect that occurs when the radio signal reaches at the receiving antenna by two or more paths. This multipath denotes the radio signal is transmitted from the transmitter, then reflected, diffracted, and scattered by the interaction objects (also known as "scatterers") presented in the channel. The multiple copies of the transmitted signal travel from several different paths, then arrive and combine at the receiver. This combination can be either constructive or destructive that can cause random fluctuations in the received signal's amplitude, phase, and angle of arrival, which is termed as multipath fading.

The fading in mobile communication can be classified into two main groups: large-scale fading and small-scale fading [8]. Literally, the large-scale denotes a large distance (hundreds of wavelengths) that the radio signal traveled. Generally, the slow dissipation of energy due to the separation of transmitter and receiver within such a large distance is defined as path loss. In the meantime, the penetration or diffraction around large objects in the actual propagation channel result in the fluctuations on the local-mean power, which are the so-called large-scale fading (or "shadowing"). Small-scale fading is used to describe the self-interference of the arrived signals from different paths with different amplitudes, delays, and phases at the receiver over a short period (on the order of $10 \mathrm{~ns}$ depending on mobility) or a travel distance on the same order of the wavelength. Fig. 2.2 illustrates an example of the large-scale fading that superimposed with the small-scale fading [9].

As shown in Fig. 2.2, path loss is modeled as the distance-dependent power 


\section{Chapter 2. Radio Propagation and Channel Modeling}

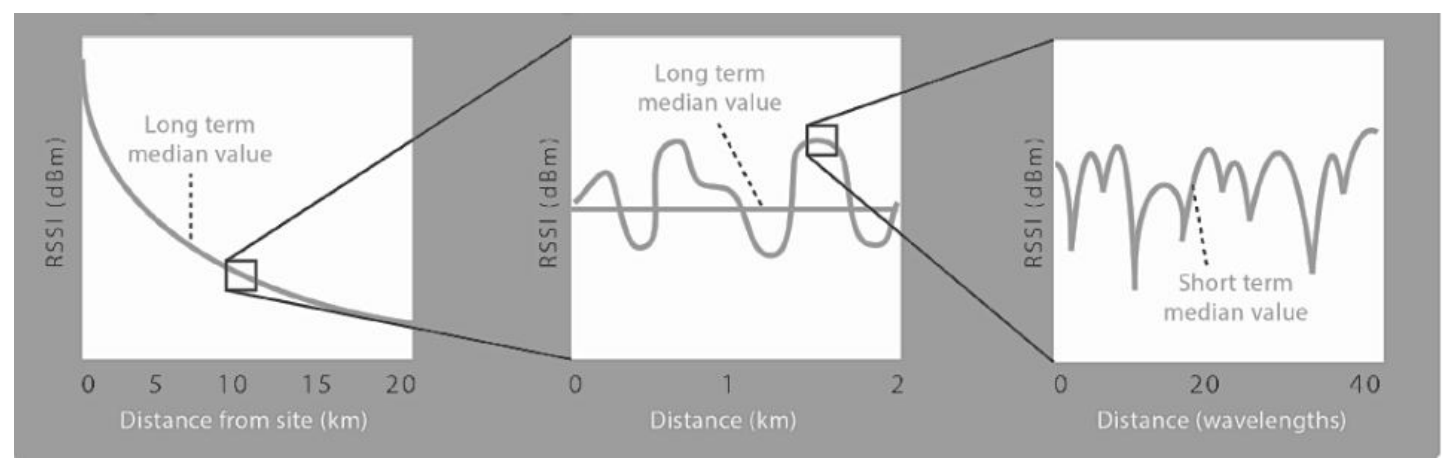

Figure 2.2. Path loss, large-scale fading, and small-scale fading.

decay of EM waves. Then, the large-scale fading is described as the local mean variation on the path loss. The small-scale fading is the rapid change in signal strength on a scale of the wavelength. As the time scale is on the order of a typical duration of physical layer communication, small-scale fading is more complex and challenging due to [10]:

- channel uncertainty: it is hard to track the channel gain precisely and require more signaling overhead;

- construction/destruction: with a certain probability, the channel gain may be deteriorated and results in an instantaneous channel capacity. But in some other momentsthe capacity may be increased when the channel gain is favorable because of the construction of multipath reception.

\subsubsection{Fading Classification}

As mentioned above, the fading can be primarily divided to the large-scale and small-scale fading based on the spatial or time scale. Whereas for practical mobile communication application, the small-scale fading depends on not only the properties of the transmitted signal (such as bandwidth $B_{s}$, symbol period $T_{s}$, etc.) but also the characteristics of the wireless channel. The small-scale fading is influenced by three foundational manifestations as follows:

Delay spread of the signal: a certain variation (spread) of the average received power as a function of the time delay. In other words, delay spread is the overall 


\section{Chapter 2. Radio Propagation and Channel Modeling}

span (i.e., from the earliest arrival time to the latest arrival time) of path delays, sometimes referred to as the excess delay spread [11].

Time variance of the channel: since the received multipath components (MPs) are highly dependent not only on the signal frequency but also on the position of the antenna [12], the significant time variation may occur due to: (i) the relative motion between the transmitter and the receiver, which results in the random frequency modulation because of the Doppler shift $f_{d}$. The Doppler shift can be positive or negative depends on the mobile receiver is moving towards or away from the transmitter; (ii) the movement of surrounding objects in the propagation environment. If the surrounding objects in the propagation area move at a greater rate than the mobile receiver, this effect dominates the small-scale fading.

Transmission bandwidth of channel: the bandwidth of a channel is used to be quantified by the coherence bandwidth $B_{c}$, which is a measure of the maximum frequency difference for which signals are slightly correlated in amplitude and as the figure of merit of system [13]. Thus, the received signal will be distorted if the transmission bandwidth is greater than the bandwidth of the multipath channel.

\section{Fading}

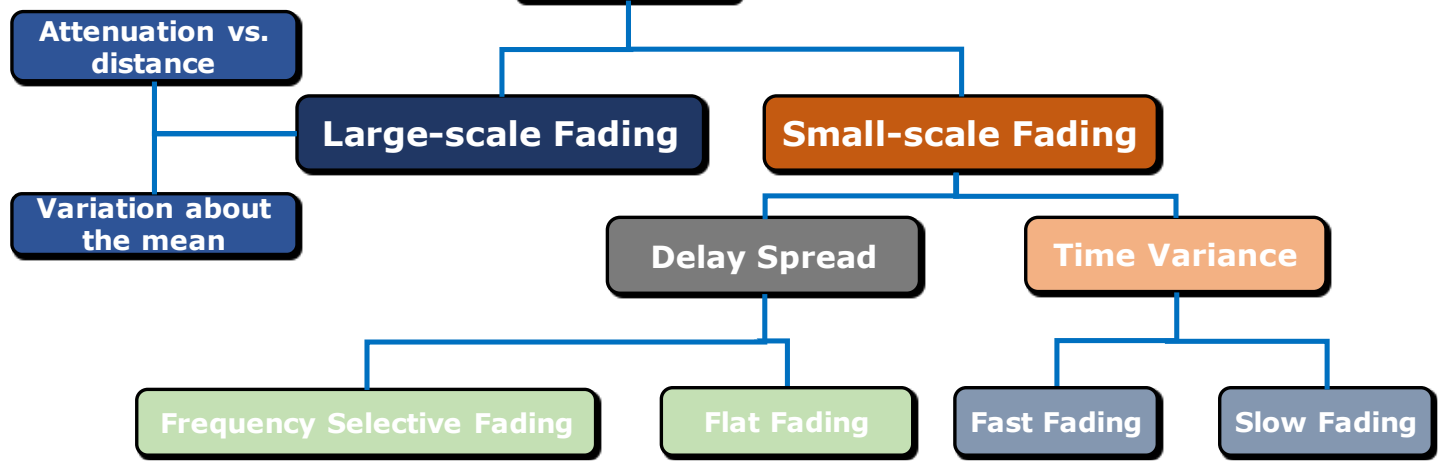

Figure 2.3. Fading classification.

In summary, depending on the nature of transmitted signal, the mobile channel, the velocity of relative motion, the delay spread of the signal and the time variation of the channel mechanisms in a mobile communication channel result in four possible distinct small-scale fading types:

- On the basis of delay spread: 


\section{Chapter 2. Radio Propagation and Channel Modeling}

- Flat fading: the bandwidth of the transmitted signal is narrower than the bandwidth of the multipath channel with a constant gain and linear phase response. The received signal strength changes with time due to fluctuations in the gain of the channel caused by multipath, but the spectrum of the transmission is preserved. Thus the flat fading channel is also known as amplitude varying channel and sometimes referred to the narrowband channel $\left(B_{s}<<B_{c}\right)$.

- Frequency selective fading: The channel induced intersymbol interference (ISI) will appear if the maximum excess delay spread $T_{m}$ exceeds the symbol time $T_{s}$. Contrary to the flat fading, the bandwidth of transmitted signal is greater than the coherence bandwidth of the channel in a frequency selective fading (i.e. $B_{s}>>B_{c}$ ). Frequency selective fading channels are also known as wideband channels and much harder to model than flat fading channels since each multipath component must be modeled and channel must be considered as a linear filter [13].

- On the basis of Doppler spread, the coherence time $T_{c}\left(T_{c}=1 / f_{d}\right)$ is a statistical measure describing the time frame over which the channel response can be considered as invariant [14]:

- Fast fading: coherence time $T_{c}$ of the channel is much smaller than symbol period of transmitted signal (i.e. $T_{c}<<T_{s}$ ), which implies the impulse response of channel changes at a fast rate during one symbol period.

- Slow fading: the coherence time is sufficiently long that the channel impulse response changes at a slower rate than the transmitted signal. In this case, the channel can be assumed invariant over any of bandwidth interval.

Eventually, the channel fading can be illustrated as the tree diagram in Fig.2.3. 
Chapter 2. Radio Propagation and Channel Modeling

\subsection{Channel Modeling}

A channel model is known as an abstract and simplified approach to mathematically demonstrates the main characters of an actual channel, and evaluates the influences on the performance of a specific wireless technology in this channel. Thus, establishing our knowledge and understanding of the behavior of how the propagation environment affects the wireless signals is of important significance, which is the primary objective of channel modeling. A logical channel modeling conduces to the design, implementation, testing, and deployment of the corresponding wireless communications system because a precise channel model can be quantitatively assessed the performance of wireless propagation under realistic conditions. Consequently, channel modeling is the premise of software simulation, channel emulation and RF planning, which can provide the supports to mitigate the impairments and distortions of radio signals in wireless communication [2].

Furthermore, as new wireless communication systems and technologies are developing in more and more specific environments and expected to provide more reliable and efficient links, existing channel models are also necessary to extend and update for new requirements, such as higher frequencies and particular propagation scenarios. For instance, radio propagations in railway environment are complex, due to the particular characteristics of the scenarios like tunnels, viaducts, and cuts. Therefore, new channel models benefit the design and development of wireless communication systems in railway.

Besides, it is extremely challenging to obtain the exact mathematical characterization of a realistic wireless channel due to the time variation and unpredictable nature. Solving Maxwells equations with proper boundary conditions that reflect all physical constraints of the propagation environment can be a possible, but the detailed knowledge of geometric and dielectric properties of the environment is considerably hard to provide [15]. Therefore, an efficient channel model should focus on describing the characters that touch the core effects on the channel propagation and ignore the rest to reduce complexity. As previously stated, the impairments and distortions of radio signals in the wireless channel can be divided into two groups: large-scale fading and small-scale fading. Consequently, a channel model that predict the mean signal strength versus the 


\section{Chapter 2. Radio Propagation and Channel Modeling}

transmitter-receiver separation distance is called large-scale propagation model, which can be used for calculating the link budget. In the meantime, the rapid fluctuations of the received signal strength over very short travel distances or short time durations are characterized in small-scale propagation models [16].

Besides, the modeling approach provides another line of thought for channel models classification. Fundamentally, the modeling approaches fall into two categories: physical models and analytical models. The physical models are established on the basis of electromagnetic wave propagation and independent of antenna configurations, such as antenna pattern, number, etc. It models the bidirection propagation between the transmitter and receiver based on the measurement or simulation in a specific scenario, which means it changes as the locations of transmitter and receiver changes. Whereas the analytical models mathematically/analytically reproduce the statistical properties of the MIMO matrix in the corresponding domain, whereas the physical propagation mechanisms are not considered [17]. In this thesis, the physical models are mainly focused by the mean of measurement campaigns. The physical channel models can be generally classified into two types [18]:

- Empirical/Statistical model: this kind of channel model is extracted from the large amounts of measurement data. The propagation characterization in the specific scenarios is modeled by the statistical analysis of the channel impulse response (CIR), and then extracting the key parameters, such as path loss, delay spread, angular spread, etc to parametrized the channel.

- Deterministic models: characterizing the physical propagation in a completely deterministic way, such as ray tracing/launching. The basic idea of these deterministic approaches is calculating the received signal from knowledge of geometry, the electrical properties of the medium of propagation, cross section of objects and antenna radiation pattern.

For measurement-based empirical channel modeling, the first step is the channel measurement (sounding) in the realistic environment. Specifically, sending a given training sequence from the transmitting system to the wireless channel, then capturing and analyzing the attenuated and distorted signal to obtain the CIR on the receiver side. The principle of channel sounding, the commonly used test 


\section{Chapter 2. Radio Propagation and Channel Modeling}

equipment, and the domestically developed channel sounders are introduced in the next chapter. Based on the measurement results, the channel can be characterized by a set of parameters: the path loss, shadowing, Rician $\mathrm{K}$ factor from the narrowband measurement; the channel transfer function in space/time/frequency domains, the resolvable angular/Doppler/delay spread for small-scale modeling. These concepts are explained in the following subsections. The ray-tracing technique as the classic deterministic modeling approach is introduced in the last subsection.

\subsubsection{Large-scale fading Models}

\section{Path Loss and Shadowing}

The Free space path loss model as described in Eq. 2.1 is the area-mean power that restricted to a clear and unobstructed direct path between the transmitter and the receiver. Whereas the long-term shadow fading is used to result in the variation in radio signal power due to the encountering with obstructions over long distance. Egli demonstrates that the received local-mean power of the radio signal fluctuates about the area-mean power with a log-normal distribution in [19]. Hence, Log-distance path loss model is a generic model and an extension to Friis Free space model and expressed as follows:

$$
P_{L}(d)[d B]=P_{\mathrm{Tx}}[d B m]-P_{\mathrm{Rx}}[d B m]=P_{0}+10 n \log _{10}\left(\frac{d}{d_{0}}\right)+X_{s}
$$

where $P_{0}$ is the path loss in $d B$ at the reference distance $d_{0}$, and $n$ is the path loss exponent, which describes the path loss trend versus the distance $d$ between transmitter and receiver. $X_{s}$ represents a zero-mean Gaussian distributed random variable in $\mathrm{dB}$ with a standard deviation $\sigma_{x}$.

In practice, $P_{0}$ can be obtained from measurements or free space assumption (friis) from the transmitter to $d$ assumption (friis) from the transmitter to $d_{0}$. The path loss exponent $n$ and standard deviation $\sigma_{x}$ can be calculated from measured 
data, using linear regression such that the difference between the measured and estimated path losses is minimized in a mean square error sense over a wide range of measurement locations and T-R separations. This model can be used in computer simulation to provide received power levels in arbitrary locations for radio coverage planning and network optimization of a communication system as shown in Fig. 2.4.

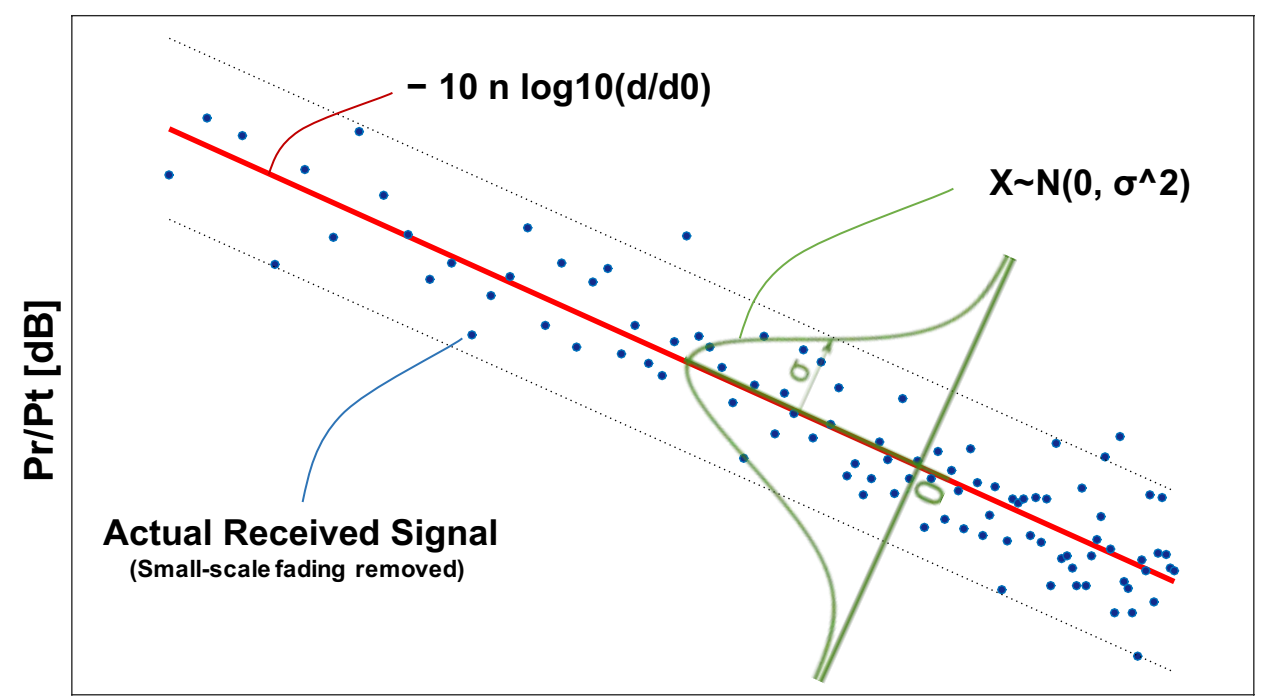

\section{Log Distance}

Figure 2.4. Log distance path loss model.

\section{Other Models}

For outdoor environments, the density of people should not be neglected. Therefore the exclusive path loss model for specific propagation scenario is helpful for optimum network design. There are several well-known empirical propagation models, such as:

Okumura Model: radio propagation model for macro cells in urban areas (no tall blocking structures).

Hata Model: the most widely used prediction model for cellular transmissions in a city environment. It is suitable for both point-to-point and broadcast transmissions and classified into three versions for Urban Areas (high-density city 


\section{Chapter 2. Radio Propagation and Channel Modeling}

included tall buildings), Suburban Areas, and Open Areas.

Lee's Model: the most popular macroscopic propagation models. It is a slopeintercept propagation prediction model. The main assumption of the model is that the propagation path loss depends on two influencing factors: the natural terrain, and the clutter from man-made structures.

COST231 Models: (i) COST-HATA Model as the extend Hata model to cover a more elaborated range of frequencies ; (ii) COST 231 Walfisch-Ikegami Model is another model for micro cells and small macro cells by combining models proposed by Walfisch and Ikegami, which introduced additional Characteristics, such as heights of building, width of roads, building separation, and road Orientation with respect to the direct radio path.

Through the comparison and analysis, it can be found log-distance path loss model is more simple, efficient, and suitable for computer implementation without the knowledge of the environment. Therefore, there is no requirement for investing time and resources in surveying building layouts. Due to model simplicity, great accuracy could not be expected. The main parameter $n$ is very sensitive to the properties of propagation environment, such as the type of construction material, type of interior, location within the building, etc. The value of $n$ equal to 2 in the free space, and normally range from 1.2 (waveguiding effect) to 6 . Also, the value of $\mathrm{n}$ depends on the way the statistical analysis on measurement data is performed [20].

\section{Link Budget}

The link budget is a written description of all the gains and losses by a transmitter during its active state. It is a convenient tool to compare power levels between different technologies and different systems. It is a way of quantifying the link performance and commonly identified by the following parameters:

- Effective Isotropic Radiated Power (EIRP): EIRP is the power radiated relative to a perfect isotropic antenna. It is the sum of available transmit power and antenna gain in $\mathrm{dBi}$, and subtracting any extra loss from cable, inefficiency, etc. EIRP defines the maximum transmit power.

- Signal to Noise Ration (SNR): SNR determines the certain error probability 


\section{Chapter 2. Radio Propagation and Channel Modeling}

given a signal modulation at the receiver. The SNR is sometimes expressed in term of energy per bit over noise power spectral density.

- Sensitivity: Sensitivity denotes the lowest power level at which the received signal may be decoded, it is usually defined as a power level above ambient noise and interferences, and depends on several parameters such as bit rate, coding, error rate. The amount by which the received power exceeds receiver sensitivity is called the link margin.

- Maximum allowable path loss: As mentioned above, the path loss is the sum of all the losses between transmitter and receiver that are not to do with the antennas or feeders, which quantifies the radio system performance. It provides a technology independent value that can be used for coverage, capacity, or other estimates.

\subsubsection{Amplitude Statistics}

As previously stated, the received signal in a realistic environment is a combination of multipath signals arriving from different directions, phases, and amplitudes. The small-scale fading distribution is a statistical characterization of the multipath fading, which describes how the received signal amplitude changes with time. The envelope of the received signal is usually modeled by a random variable with a certain probability distribution.

\section{Rayleigh Distribution}

Rayleigh fading distribution is widely used to model a small-scale fading and describes a statistical time-varying model for the propagation. To model a multipath environment with no line of sight (NLOS) path, the time variant channel impulse response $h(t)$ in a flat fading channel is given by [21]:

$$
h(t)=r(t) e^{j \phi(t)}=x(t)+j y(t)
$$

where $r(t)$ denotes the magnitude or envelope, and $\phi(t)$ is the phase rotation, $x(t)$ and $y(t)$ represent the in-phase (I) and quadrature (Q) component, respectively. 


\section{Chapter 2. Radio Propagation and Channel Modeling}

If the signal follows a complex Gaussian distribution, in particular, both random processes $x(t)$ and $y(t)$ are statistically independent Gaussian distributed with the same variance and zero mean, then their joint probability density function (PDF) can be represented by:

$$
P(x, y)=P(x) P(y)=\frac{1}{2 \pi \sigma^{2}} e^{-\frac{x^{2}+y^{2}}{2 \sigma^{2}}}
$$

If differential areas is transformed to magnitude and phase through $d x d y=$ $r d r d$, the joint PDF of $r(t)$ and $\phi(t)$ can be expressed as:

$$
P(r, \phi)=\frac{r}{2 \pi \sigma^{2}} e^{-\frac{r^{2}}{2 \sigma^{2}}}, \quad r \in[0, \infty], \phi \in[-\pi, \pi],
$$

Since the random variables $r$ and $\phi$ are statistically independent, i.e. $P(r, \phi)=$ $P(r) P(\phi)$, the PDF of $r$ and $\phi$ can be obtained as follows:

$$
P(r)=\int_{-\pi}^{\pi} \frac{r}{2 \pi \sigma^{2}} e^{-\frac{r^{2}}{2 \sigma^{2}}} d \phi=\frac{r}{\sigma^{2}} e^{-\frac{r^{2}}{2 \sigma^{2}}}
$$

and

$$
P(\phi)=\int_{0}^{\infty} \frac{r}{2 \pi \sigma^{2}} e^{-\frac{r^{2}}{2 \sigma^{2}}} d r=\frac{1}{2 \pi}
$$

Eventually, the envelope and phase of the received signal at any time instant undergoes a Rayleigh probability distribution and a uniform distribution, respectively.

\section{Rician Distribution}

In some propagation scenarios, there is a dominant stationary signal component $A$ present, such as the LOS path, the signal amplitude is given by $r=$ $\sqrt{(A+x)^{2}+y^{2}}$, and the small-scale fading fits with Rician distribution, thus the PDF of magnitude $r$ is given by:

$$
P(r)=\frac{r}{\sigma^{2}} e^{-\frac{r^{2}+A^{2}}{2 \sigma^{2}}} I_{0}\left(\frac{r A}{\sigma^{2}}\right)
$$

where $\sigma^{2}$ represents the variance of the random component, $A$ is the peak am- 


\section{Chapter 2. Radio Propagation and Channel Modeling}

plitude of the dominant component with power $A^{2} / 2, I_{0}(z)$ is the 0 -th-order modified Bessel function of the first kind $\left(I_{0}(x)=\frac{1}{2 \pi} \int_{0}^{2 \pi} e^{x \cos \theta} d \theta\right)$ [11].

Then the Rician K-factor as the ratio of the deterministic to the random component of the process is defined as:

$$
K[d B]=10 \log \left(\frac{A^{2}}{2 \sigma^{2}}\right)
$$

As the amplitude of dominant path decrease $(A \rightarrow 0), K[d B] \rightarrow \infty$, the Rician distribution degenerates to a Rayleigh distribution [22].

\section{Nakagami Distribution}

Despite the Rayleigh and Rician distributions are well fitted with the signal envelopes after fading channels in many actual cases, the Nakagami distribution is proved to provide a better match with the empirical data [23] [24]. Compared with Rician distribution, Nakagami distribution as a more general fading distribution does not need to assume a direct path condition, and use a parametric gamma distribution-based density function to describe the empirical data and get approximately distribution [25]. The PDF of Nakagami distribution is represented as:

$$
P(r)=\frac{2}{\Gamma(m)}\left(\frac{m}{2 \sigma^{2}}\right)^{m} r^{2 m-1} e^{-\frac{m r^{2}}{2 \sigma^{2}}}, \quad r \geq 0 ; m \geq \frac{1}{2}
$$

where $\sigma^{2}=\frac{1}{2} \mathrm{E}\left[r^{2}\right]$ is the mean-squared value of the random variable, $\Gamma()$ is the Gamma function. $m=\frac{\mathrm{E}^{2}\left[r^{2}\right]}{\operatorname{Var}\left[r^{2}\right]}$ is the shape factor or fading figure, which is used to describe the fading degree of the multipath fading channel. In the special case $m=1 / 2$, the PDF of Nakagami distribution reduces to a one-side Gaussian distribution; for $m=1$, Rayleigh fading is recovered. With proper adjustment of the parameters, Nakagami can also tightly fit Rician and lognormal distribution [11]. The Nakagami and Rice distributions can approximate each other by [26]:

$$
m=\frac{(K+1)^{2}}{2 K+1}
$$

which tends to $m=K / 2$ for large Rician K-factor. 
Chapter 2. Radio Propagation and Channel Modeling

\section{Weibull Distribution}

Weibull distribution represents another general distribution, which also included Rayleigh as a special case. Here the signal envelop is given by $r=\sqrt[\beta]{x^{2}+y^{2}}$, and the corresponding PDF is expressed as [27]:

$$
P(r)=\frac{\beta}{2 \sigma^{2}} r^{\beta-1} e^{-\frac{r^{\beta}}{2 \sigma^{2}}}, \quad r \geq 0
$$

where $\sigma^{2}=\frac{1}{2} \mathrm{E}\left[r^{2}\right], \beta$ is the shape factor, which determines to the fading severity, when the $\beta=2$, Weibull distribution degrades to Rayleigh distribution, for $\beta<2$, the fading is severer than the Rayleigh fading. Nowadays, Weibull distribution is one of the most widely used tool for reliability engineering and failure data analysis. In mobile communication, the Weibull distribution is also attracting more and more interest, since it exhibits good fits to experimental measurements data in both indoor and outdoor propagation environments [28].

\subsubsection{Time Dispersion}

In mobile communication, the mobile channels can be modeled as a linear digital filter. Thus its impulse response includes smallest variations of the signal and the output of the channel [29]. The impulse response provides a wideband characterization of the propagation channel and contains the essential information to analyze and compare the radio propagation in a particular mobile channel condition [30].

For recalling the time dispersion, the multipath propagation in Fig. 2.1 can be analyzed as an example. When a signal is transmitted from single transmission antenna, the signals travel through various propagation paths with different travel distances and then arrive the receiving antenna. Assuming there are four transmission paths, which could be resolved in the receiver side, received signal in each path has an amplitude $a_{i}$ and a delay time $\tau_{i}(i=0,1,2,3)$. Then the received signals can be represented as shown in Fig. 2.5. As those arrival times on the axis of time shows, a certain spread of various delays can be found, which is the time dispersion phenomenon, and such a spread is the so-called "Delay Spread". Then the channel impulse can be represented by: 


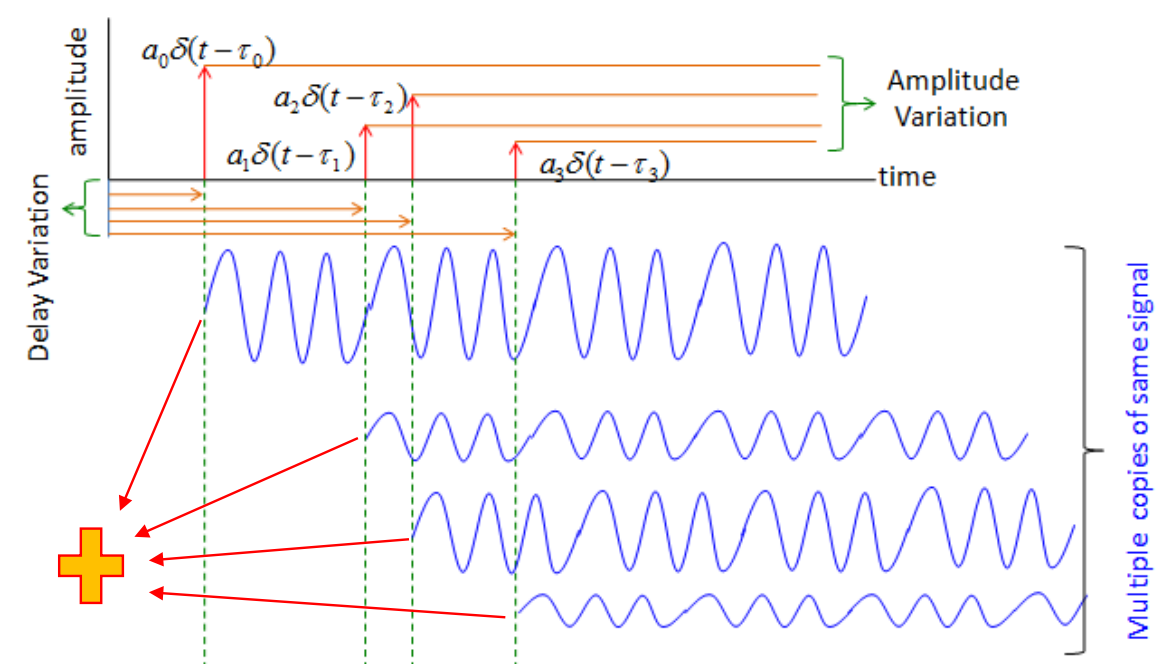

Figure 2.5. The delay spread conception, via: www.sharetechnote.com.

$$
h(t)=\sum_{i=0}^{3} a_{i} \delta\left(t-\tau_{i}\right)
$$

Arrival times of MPCs are affected by the location of the interacting objects in the channel. In the extreme with a simple geometrical relationship, e.g. each MPC only interacts once in its traveling path, then the position of the interacting object uniquely determines the excess runtime. Assuming the interacting objects in an indoor environment are distributed uniformly in a plane, then the multipath arrival time is stated as a random process based on Poisson distribution, which therefore means the inter-arrival times between MPCs follow the exponential distribution [31].

However, interacting objects are not distributed uniformly in the practical environment and normally clustered in a group. In other words, groups are separated in space with certain distances, and objects in a group are close together. For instance, a television cabinet with TV on it. The group of objects can be approximately translated into clustering of MPCs. Therefore, the impulse response in Eq. 2.13 can be extended and written as follows [31]: 


$$
h(t)=\sum_{l=0}^{L-1} \sum_{k=0}^{K-1} a_{k, l} \exp \left(j \phi_{k, l}\right) \delta\left(t-T_{l}-\tau_{k, l}\right)
$$

Where $L$ is the number of clusters, and $K$ is the number of MPCs within a cluster; $T_{l}$ is the arrival time of the $l$-th cluster; $a_{k, l}, \phi_{k, l}$, and $\tau_{k, l}$ are the amplitude, phase, and delay of the $k-t h$ component within the $l-t h$ cluster, respectively. Formally, Eq. 2.14 splits the summation in Eq. 2.13 into a double summation, and thus establish a deeper relationship between the physics of the environment and the impulse response. Hence, the joint statistics of all the MPCs within a certain number of clusters can be easily described. Also, the statistics of the clusters themselves can be easily modeled.

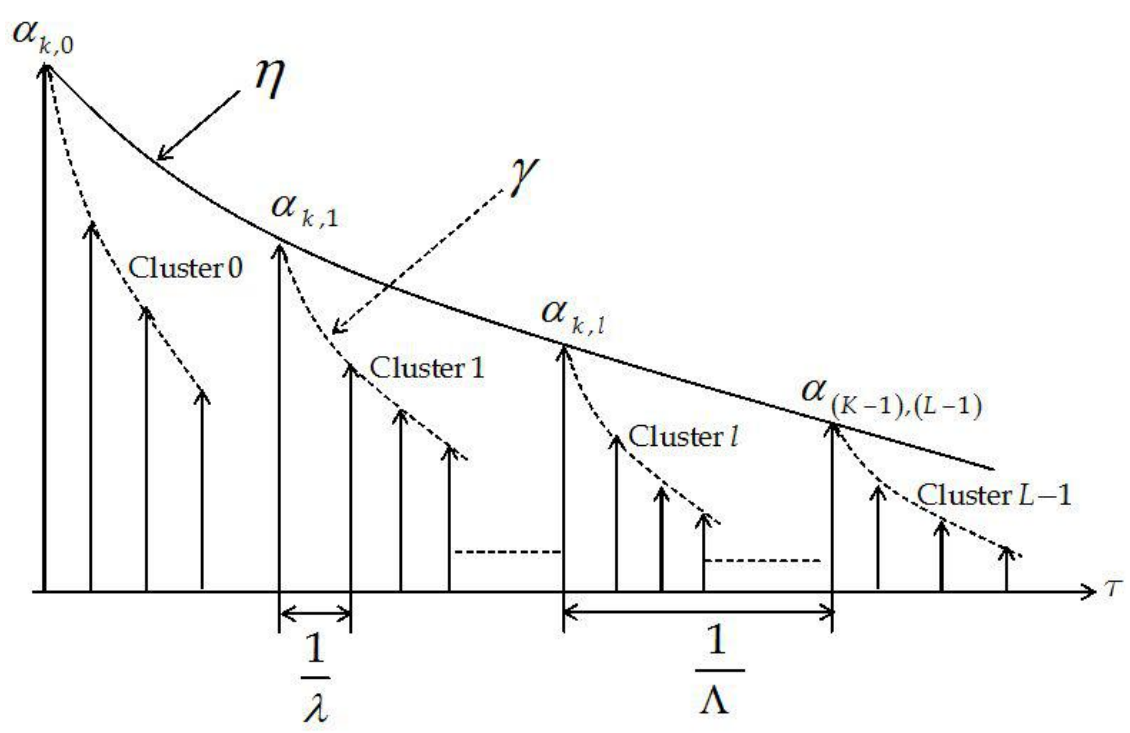

Figure 2.6. The SalehValenzuela model.

\section{Saleh-Valenzuela Channel Model}

The principle of Saleh-Valenzuela ( $\mathrm{S}-\mathrm{V})$ channel model [32] is shown in the Fig. 2.6 [33]. This model assumes an ideal situation that the small-scale amplitude fading statistics follow a Rayleigh distribution, and the corresponding phase angles are independent uniform random variables over $(0,2 \pi)$. The power decays 


\section{Chapter 2. Radio Propagation and Channel Modeling}

exponentially with cluster decay rate $\eta$ as well as excess path delay rate $\gamma$ within a cluster. S-V model suggests that the cluster arrival times $T_{l}$ and path arrival times within a cluster are random process based in Poisson distributions, with the inter-arrival rate $\Lambda$ and $\lambda$, respectively. In other words, the inter-arrival times of the clusters and MPCs are also exponentially distributed. Hence, the channel can be defined by four parameters [33]: The cluster arrival rate $\Lambda$, the path arrival rate $\lambda$ within a cluster, the cluster decay rate $\eta$, path decay rate $\gamma$.

The IEEE TG3a has proposed modified model for IEEE 802.15.3a UWB channel, which suggests the small-scale amplitude statistics $a_{k, l}$ follow a lognormal distribution. Then, IEEE TG4a suggests to model the MPCs arrival time with mixtures of two Poisson processes, and the small scale fading for the multipath gain magnitude $a_{k, l}$ is modeled as a Nakagami-m distribution in the $802.15 .4 \mathrm{a}$ UWB channel model. This model is a generalized version of S-V model for outdoor and indoor environments with longer operating range [34].

Actually, clustering is not the unique phenomenon that only occurs in UWB channels. Depending on the propagation environment and the bandwidth of the system, three cases can be defined in Table 2.1 [31]

Table 2.1. Three cases for the cluster and multipath resolution.

\begin{tabular}{l|c|c|l}
\hline Cases & Clusters & MPCs & Remarks \\
\hline$B<1 / \tau_{\max }$ & Unresolvable & Unresolvable & Bandwidth is too small \\
\hline$\Lambda<B<\lambda$ & Resolvable & Unresolvable & $\begin{array}{l}\text { Outdoor: B }>1 \mathrm{MHz} \\
\text { Indoor: B }>10 \mathrm{MHz}\end{array}$ \\
\hline$B>\lambda$ & Resolvable & Resolvable & Bandwidth is sufficiently large \\
\hline
\end{tabular}

Consequently, the number of the resolvable clusters and/or MPCs depends on the environmental parameters and measurement bandwidth. The most current broadband measurements can achieve the resolvable clusters and unresolvable MPCs. As the measurement bandwidth improves significantly, a sparse impulse response contains delay bins without MPCs is expected to obtain.

\section{Power Delay Profile}




\section{Chapter 2. Radio Propagation and Channel Modeling}

For small-scale channel modeling, the power delay profile (PDP) of the channel is found by taking the spatial average of $|h(t)|^{2}$ over a local area. The PDP provides a distribution of the average power for the received signal over individual path, which is represented by the relative power of each path related to that of the earliest path [35]. If several local area measurements of $|h(t)|^{2}$ are made in different position, an ensemble of PDPs can be built. Thus, the power profile of the multipath channel can be defined based on the Eq. 2.13 as:

$$
\phi(t)=\sum_{i=0}^{N}\left|a_{i}\right|^{2} \delta\left(t-\tau_{i}\right)=\sum_{i=0}^{N} g(i) \delta\left(t-\tau_{i}\right)
$$

Where $N$ is the maximum number of multipath that system can detect, $g(i)=$ $\left|a_{i}\right|^{2}$ and $\tau_{i}$ are the power gain and delay time of the $i^{t} h$ path, respectively.

The PDPs are used to measured by the broadband channel sounding techniques, which will be described in the following chapter. The extent of multipath effects on radio channel can be clearly determined by the time delay spread. Since the time dispersion is highly dependent on the geometric construction and dielectric properties of materials, Certain channel parameters that grossly quantify the multipath channel can be extracted according to the PDP curve as follows:

- First arrival delay $\left(\tau_{0}\right)$ : the first arrived component, which is typically the most significant component that is judged as the LOS path. For the NLOS case, the first arrival delay might be the first reflected ray. Nevertheless, this delay has traveled the minimum propagation distance, which is used to serve as a reference, and the delay component follows it is called the excess delay.

- Maximum excess delay $\left(\tau_{m}\right)$ : the time delay during which multipath energy falls to $X d B$ below the maximum. $X d B$ is the threshold level dependent on the receiver sensitivity and noise floor at the receiver. Thus the maximum excess delay is defined as $\tau_{m}-t_{0}$, which describes the temporal extension of the multipath reception above a particular threshold $X d B$.

- Mean excess delay $(\bar{\tau})$ : the first moment of the power delay profile is defined 


\section{Chapter 2. Radio Propagation and Channel Modeling}

as:

$$
\bar{\tau}=\frac{\sum(\tau) \phi(\tau)}{\sum \phi(\tau)}
$$

- $R M S$ delay $\left(\sigma_{\tau}\right)$ : the square root of the second central moment of the power delay profile. It is the standard deviation about the mean excess delay, which is similar to the standard deviation of a statistical distribution. For a discrete channel, RMS delay is given by:

$$
\sigma_{\tau}=\sqrt{\overline{\tau^{2}}-(\bar{\tau})^{2}}, \text { where } \overline{\tau^{2}}=\frac{\sum \tau^{2} \phi(\tau)}{\sum \phi(\tau)}
$$

RMS delay quantifies the strength of inter-symbol interference (ISI) by the ratio of RMS delay spread and symbol time duration, which determines the complexity of the equalizer required at the receiver.

- Coherence Bandwidth As discussed above, the coherence bandwidth $B_{c}$ can be used to characterize mobile channel in the frequency domain. It is generally obtained by the measure of a frequency range that the channel keeps in "flat", where all the spectral components have close gain and linear phase [29]. The coherence bandwidth is obtained with respect to RMS delay [36]:

$$
B_{c, 90} \approx \frac{1}{50 \sigma_{\tau}}
$$

when the correlation threshold is 0.9 (i.e. $90 \%$ coherence bandwidth); and

$$
B_{c, 50} \approx \frac{1}{5 \sigma_{\tau}}
$$

for $50 \%$ coherence bandwidth approximation.

Note there is no global agreement on the exact form of above relations [30]. Typically, when the symbol time period is greater than ten times the RMS delay spread, no ISI equalizer is needed in the receiver [37]. 
Chapter 2. Radio Propagation and Channel Modeling

\subsubsection{Tapped Delay Line Model}

A common way to model the multipath channel is as a series of amplitude weighted delayed copies of the input signal, which is the so-called "tapped delay line (TDL)" model. A TDL model discretely characterizes the wireless channel by the channel impulse response (CIR) (or the Fourier transform of CIR), which is a linear, finite impulse response (FIR) filter model as illustrated in Fig. 2.7. Thus, the output of this channel model is given by:

$$
y(t)=x(t) * h(t, \tau)+n(t)=\sum_{i=0}^{L-1} h_{i}(t) x\left(t-\tau_{i}\right)+n(t)
$$

where $*$ represents convolution, $n(t)$ is the additive noise, $x(t)$ and $y(t)$ denote the input and output of the TDL model, respectively. $\tau_{i}(t)$ and $h_{i}$ are the i-th channel tap delay time and tap weight, respectively. $L$ is the number of taps, which is the maximum number of time-resolvable multipath components. Each tap can be considered as an effective delay bin that sum of all signal received from different paths over the duration of that bin. This is analogous to discrete frequency spectra as each frequency bin is the combination of all received signal over the bandwidth that bin occupies [38]. Hence, the output of a TDL model is the combination of the input signal delay by incremental amounts and multiplied by a weight function. As a common approach, the delay time between taps $\tau_{1}, \tau_{2}, \ldots \tau_{L}$ they are equally distributed with respect to the symbol duration of the transmitted signal. However, they are somewhat irregular in the actual cases. Thus delay times in this model are not strictly equal [39]. The delay time between taps and their relative magnitude determine the frequency selectivity of the channel and the severity of ISI. The strength of time-varying taps provides the large scale attenuation and the statistical approximation of Doppler spreading and non-resolvable multipath effects to characterize the small-scale fading. The changes of tap weights affect the coherence time, Doppler spread, and higher-order statistics, such as the average fade duration [40]. For a time-varying channel, the $i^{\text {th }}$ tap weight is given by: 


$$
h_{i}(t)=a_{i}(t) e^{j \phi_{i}(t)}
$$

where $a_{i}$ and $\phi_{i}$ represent the amplitude and the phase information of $i^{\text {th }}$ resolved delay bin, respectively. Then the CIR for a TDL model can be expressed as follows [41]:

$$
h(t, \tau)=\sum_{i=0}^{L-1} a_{i}(t) e^{j\left[\omega_{D}, i(t)\left(t-\tau_{i}(t)\right)-\omega_{c}(t) \tau_{i}(t)\right]} \delta\left[\tau-\tau_{i}(t)\right]
$$

where $\omega_{c}(t)$ is the radian carrier frequency and $\omega_{D, i}$ denotes the Doppler shift of $i^{\text {th }}$ tap, $\tau_{i}(t)$ is the time-varying delay of $i^{\text {th }}$ tap.

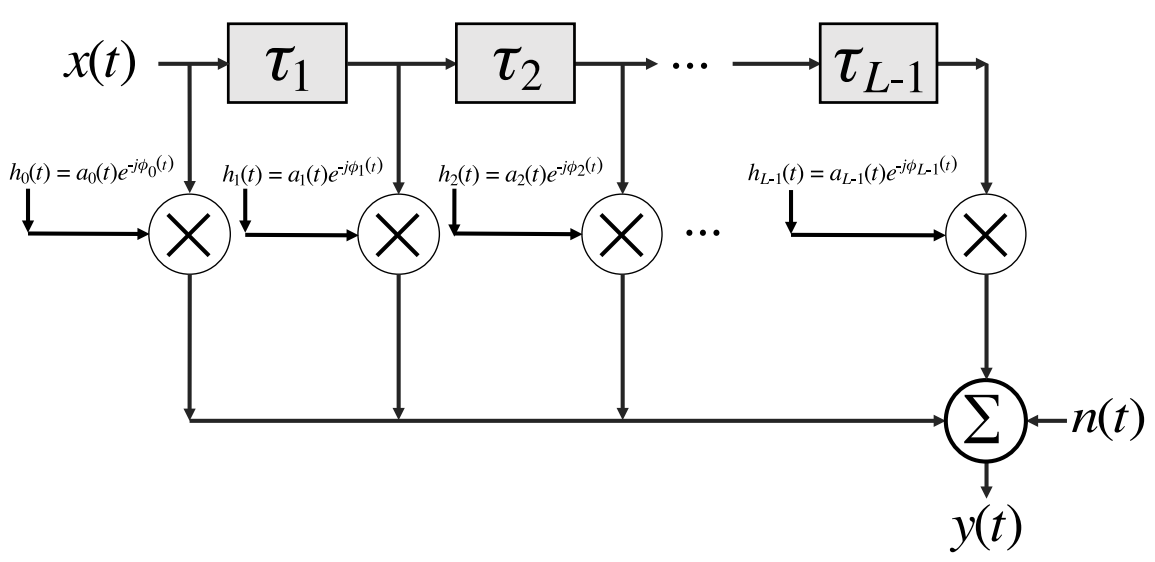

Figure 2.7. Tapped delay line model.

For a time-invariant channel, the multipath structure does not vary with time. Thus the amplitude and delay spread keep stationary either. If the delay spread is considerably large than the signal symbol duration, the amplitude and phase of the radio signal will be distorted as the frequency components experience different phase shifts along the different paths, and finally, results in a frequency selective fading. The channel capacity significantly depends on the profile of the channel, the number of channel taps, and the tap correlation profile.

In addition, the number of channel taps is commonly is determined by the product of delay spread and the bandwidth of the transmitted signal [42]. Some researchers adopt the maximum delay spread $\tau_{m}$ to obtain the maximum number 


\section{Chapter 2. Radio Propagation and Channel Modeling}

of channel taps $L$ by $L=\tau_{m} W$, where $\tau_{m}$ and $W$ denote the maximum delay spread and the bandwidth of the transmitted signal [39] [18]. On the other hand, authors in [43] [44] [45] state the RMS delay spread as the "effective delay" should be used to determine the $L$ as follows [41]:

$$
L=\left[\frac{R M S-D S_{\max }}{\text { Delay Resolution }}\right]+1=\left[\frac{\operatorname{Max}\left(\sigma_{r m s}\right)}{T_{c}}\right]+1
$$

where $T_{c}$ is the symbol rate (chip duration) and [ ] represents the rounding function.

Moreover, a switch function $z_{i}(t) \in 0,1$ has introduced for V2V channel modeling in [46] to describe the "birth" or "death" process of $i^{\text {th }}$ tap in TDL model.

\subsubsection{Angular Spread}

The angular spread refers to the spread of angle of incidence to the receiving antenna or antenna array. The angular spread results in space selective fading, which causes a variation of the signal amplitude according to the location of the antenna. Space selective fading is characterized by the coherence distance, which is related to how far the antennas need to be separated with a low correlation coefficient. For instance, in the design of a MIMO system, the highest capacity can be achieved when the signals at the antenna elements are decorrelated. The coherence distance $D_{c}$ defines that any physical position separate by $D_{c}$ have an essentially uncorrelated received signal amplitude and phase. An approximate rule of thumb relates to the wavelength and the RMS AoA is given by [47]:

$$
D_{c} \approx \frac{0.2 \lambda}{\theta_{r m s}}
$$

Eq. 2.24 shows that wireless systems at a higher frequency (shorter wavelength) indicate shorter coherence distances. In this case, the antenna arrays can be effectively employed with a rich diversity. Thus, characterization of angular spread is especially important for multi-antenna system development. 


\section{Chapter 2. Radio Propagation and Channel Modeling}

\subsubsection{Doppler Spread}

Delay spread and coherence bandwidth describe the time dispersive nature of the channel in a small-scale local area. Yet, the relative movement between the transmitter and receiver, or the moving objects in the channel cause a change in the perceived frequency, which is the so-called Doppler effect. Thus, another aspect in wireless communication is the time rate of changing in the wireless timevarying channel. In the time domain, Coherence time defines the time duration over which the channel impulse response is considered to be stationary. In the frequency domain, the Doppler effect is described by the Doppler spread. Doppler spread is a measure of the spectral broadening and describes how fast transmitter, receiver, and scatterers in-between are moving. A slowly changing channel has a large coherence time and a small Doppler spread. When a pure sinusoidal tone of frequency $f_{c}$ is transmitted in a time-varying channel, the received signal spectrum have components in the range of $f_{c} \pm f d$, where $f_{d}$ is the Doppler shift. $B_{D}$ is defined as the range of frequencies over which the received Doppler spectrum is essentially non-zero [48]. The instantaneous Doppler shift depends on the wavelength $\lambda$, the maximum relative radial speed $v_{m}$ and wave incident angle $\theta$ as follows:

$$
f_{d}=\frac{v_{m} \cos \theta}{\lambda}
$$

And the Doppler spread is defined as twice the maximum Doppler shift: $B_{D}=$ $2 f_{m}$, where $f_{m}=v_{m} / \lambda$ is the approximation of the maximum Doppler shift at the maximum speed of $v_{m}$. Coherence time $T_{c}$ is then given by the reciprocal of Doppler spread:

$$
T_{c}=\frac{1}{B_{d}}=\frac{c_{0}}{v_{m} f_{c}}
$$

where $c_{0}$ is the velocity of light. Assuming that paths of equal average amplitude and random phases arrive at the mobile from random directions in azimuth (Clarke's model), and the coherence time is defined as the time over which the time correlation function over $50 \%$, then the coherence time is approximately given by: 


$$
T_{c, 50} \approx \frac{9}{16 \pi f_{m}}
$$

Moreover, a popular rule of thumb is defined by [48]:

$$
T_{c} \approx \frac{1}{f_{m}} \sqrt{\frac{9}{16 \pi}} \approx \frac{0.423}{f_{m}}
$$

\subsubsection{Deterministic Modeling: Ray Tracing}

The deterministic modeling dedicates to determine all the contributing propagation mechanisms by employing the EM computation in a mathematically reproduced environment. The commonly used deterministic modeling approaches include: ray tracing (RT) [49], Method of Moments (MoM) [50], and FiniteDifference Time Domain (FDTD) [51]. Although MoM and FDTD have obtained high accuracy for the near-field simulation, their application is limited by the high computational complexity.

RT algorithm has better general applicability by using Geometrical Optics (GO) theory to treat reflection and transmission on plane surfaces and diffraction on rectilinear edges. It aims to determine all the contributing propagation mechanisms at the receiver side: such as the LOS component if it is present, reflected, refracted, diffracted, and the scattered components. For applying the ray tracing, a "ray" has to obey the rules as follows [52]:

- A ray travels in a straight path in homogeneous medium.

- It obeys the laws of reflection, diffraction, and scattering.

- A ray is treated as a tube (surrounding a central ray) in which the energy is contained and propagated.

Essentially, the path of the each ray is determined by the Fermat's principle of least time, which states the route of the ray is the path that consumes the least traveling time from the transmitter to the receiver. On the basis of this principle, the laws of reflection, refraction, and diffraction can be derived. Then the most simple ray tracing for the reflection path is determined by the image theory. The image theory can be easily described in three steps for a certain locations of 


\section{Chapter 2. Radio Propagation and Channel Modeling}

transmitter and receiver: first, locating the image of receiver with respect to the planar reflection surface; next, connecting the image of receiver and transmitter in a straight line intersects with the reflection surface; eventually, the path from the transmitter to the intersecting point, and then reflected from the intersecting point to the receiver compose a simple reflection path. Multiple reflections can also be determined based on this method. However, this method can be computationally inefficient in a complex scenario with lots of reflection surface. The Shooting and Bouncing Ray (SBR) method can offer a faster computation speed with lower accuracy. This method and the hybrid method with image theory is explained in detail in [52].

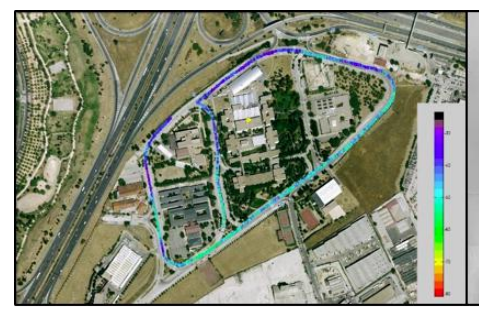

(a)

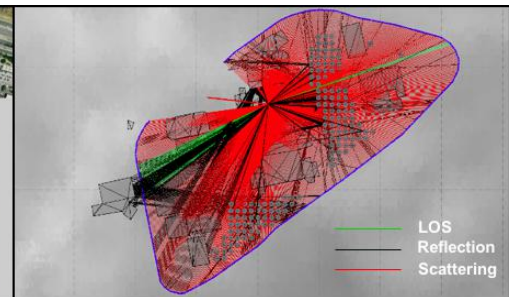

(b)

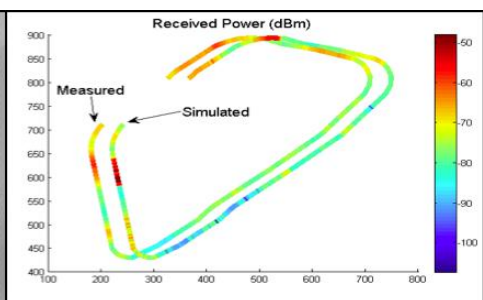

(c)

Figure 2.8. A ray tracing example: (a) propagation scenario: UPM Campus Sur, Carretera de Valencia, km. 7, Madrid, Spain; (b) ray tracing illustration; (c) comparison with the measurement results.

An example of ray tracing procedure in UPM campus is illustrated in Fig. 2.8. The geometric information of the test environment in Fig. 2.8 (a) is mathematically reproduced in Fig. 2.8 (b). Then the LOS, reflection, and diffraction paths are defined by the tracing algorithm in the ray tracer. The propagation coefficients in every path with different propagation way are then computed by EM theory. Eventually, the channel transfer function can be obtained by combining the behavior in each individual paths. The simulation results can be compared with the measurement results as shown in Fig. 2.8 (c), for tuning or improving the ray tracer. 


\section{Chapter 2. Radio Propagation and Channel Modeling}

\subsection{Discussion}

In this chapter, the essential concepts for representation of the radio channel in time/frequency/space dimensions are summarized. Generally speaking, the radiated radio wave experiences the different levels of energy dissipation for propagation in different propagation mediums. In actual environments, this dissipation is usually shadowed by the prominent obstacle contours such hills, buildings, etc., and results the transmitted signal is attenuated along the distance and accompanied by the large-scale fading. Besides, small-scale fading refers to the rapid fluctuation of the amplitude of a radio signal over a short period time or travel distance that can be occurred due to the multipath propagation. The envelope of the multipath received signal can be statistically described by Rayleigh/Rician distribution depends on the absence/presence of LOS path or dominant component. Besides Rayleigh and Rician, other distributions such as Nakagami and Weibull include Rayleigh as a special case are commonly used to generally model the small-scale amplitude. Multipath propagation refers to the transmitted signal traveling with different paths due to the reflection/diffraction/scattering, and arriving the receiver side at different times. Multipath propagation causes multipath interference including constructive and destructive interference and phase shifting of the signal. In the time domain, the multipath delays result in the time dispersion or echoes. In the meantime, the mobile receiver or the movement of surrounding objects lead to varying Doppler shifts in the frequency domain on signals at different paths. The key parameters for characterizations of multipath propagation in time and frequency domain are described in Fig. 2.9.

\begin{tabular}{l|l}
\hline Multipath $\rightarrow$ Time Dispersion & Channel Variability $\rightarrow$ Frequency Dispersion \\
Max. excess delay: $\tau_{m}$ & Doppler Spread $: B_{D}$ \\
Mean excess delay $: \bar{\tau}$ & $\begin{array}{l}\text { Max. Doppler shift }: f_{m} \\
B_{D}=f_{m}\end{array}$ \\
RMS delay $: \sigma_{\tau}$ & Coherence Time \\
\hline Coherence Bandwidth & Coherence time $: T_{c}=\frac{1}{B_{D}}=\frac{0.5}{f_{m}}$ \\
Function of channel impulse response & Time Correlation $>50 \%: T_{c, 50}=\frac{9}{16 \pi f_{m}}$ \\
Frequency correlation $>90 \%: B_{c, 90}=\frac{1}{50 \sigma_{\tau}}$ & Practical rule of thumb $: T_{c}=\frac{0.423}{f_{m}}$ \\
\hline Frequency correlation $>50 \%: B_{c, 50}=\frac{1}{5 \sigma_{\tau}}$ &
\end{tabular}

Figure 2.9. Time and frequency dispersion.

Regarding channel modeling, a complete channel model development is not 


\section{Chapter 2. Radio Propagation and Channel Modeling}

only the sounding process but also the post-processing on the RAW data for data reduction and extracting the channel parameters of interest as illustrated in Fig. 2.10. The high-resolution parameter estimation algorithms, such as SAGE and RiMAX applying on the measurement data offers an insight into radio propagation mechanisms. Follow the parameters extraction, a judgment on the key parameters is essential. The extensive channel sounding may helpful for the fine tuning to formulate a more precise or abundant channel model. The key parameters extract from narrowband measurement/simulation, such as the path loss, shadowing, Ricain $\mathrm{K}$ factor determines the radio coverage and quality of the wireless system. Wideband modeling requires the channel characterizations in time, frequency, and space dimensions. The extracted delay/Doppler/angular spread are very critical for the design and development of a high capacity wireless communication system.

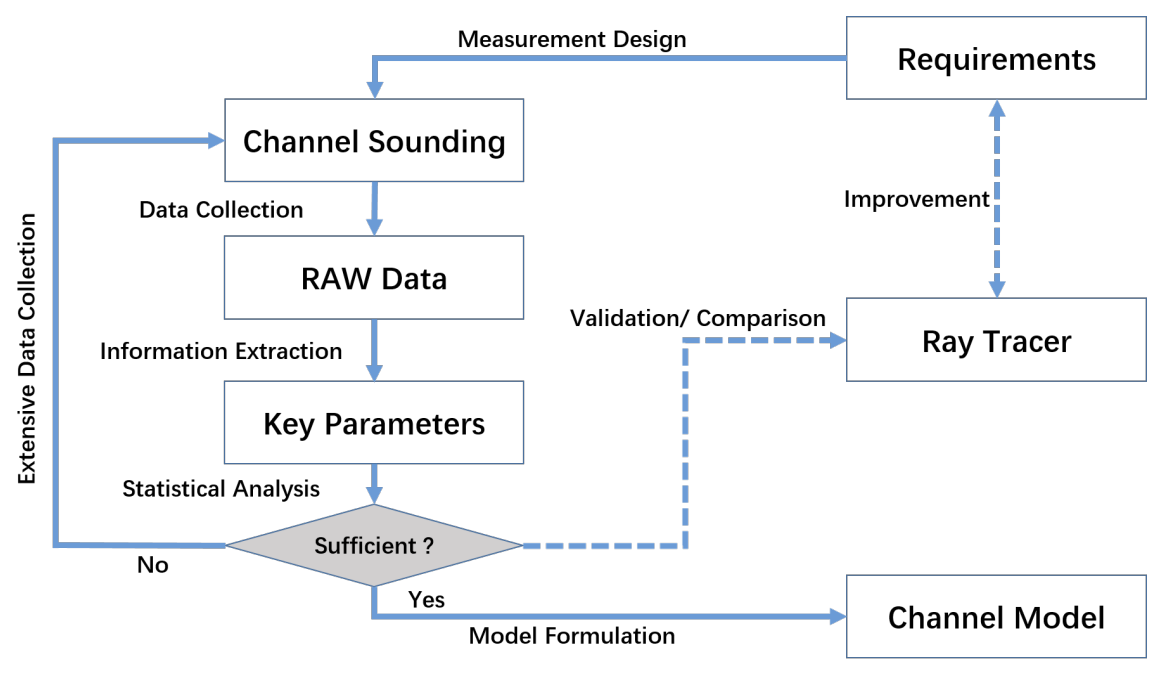

Figure 2.10. Flow chart of the channel model development.

On the other hand, the EM field could be predicted by the Geometrical or Uniform Theory of Diffraction (GTD/UTD) on the basis of the appropriate description of the EM environment (geometry, material properties, etc.), as explained in subsection 2.2.7. In this case, the empirical results can be used to compare with simulation-based (e.g. ray tracing) results for tuning and verifying between the measurement design and the ray tracer. 


\section{Chapter 3}

\section{Channel Measurement Systems}

For channel modeling purpose, the simulation-based approach such as ray tracing may have some advantages of less cost and time-consuming, relative to measurementbased methods. However, an acceptable simulation-based model requires a large number of environment details, such as the geometric information, and material properties. These kinds of prior information result in the particularly heavy computation and limit the general applicability of simulation-based approach in channel modeling. Therefore, measurement-based methods are widely for channel characterization and validation of simulation results [2]. Since the real wireless channel determines the ultimate performance limits of the wireless communication system, propagation measurements and empirical channel modeling are indispensable for wireless system design and network planning. For instance, the path loss and fading of the channel determine the coverage of cellular communication. Moreover, the key features of the wideband channel such as the channel frequency selectivity, multipath delays, directivity, polarimetric properties, and interferences significantly affect the channel capability to support high and wideband data rates [53].

Based on the systematic requirement analysis, the channel measurements can be conducted through a suitable testbed system. Abstractly, the measurement testbed transmits a "sounding" signal excites the channel, and "listening" the response of the channel on the receiver side. This measurement process designed for the channel characterization is the so-called "channel sounding", and the testbed system employed is the "channel sounder". 
After a brief overview of the narrowband and wideband channel sounding techniques, three common types of wideband channel sounder are reviewed in Sect. 3.1. Next, the high-ended channel sounder and portable channel sounder designed and developed by our research group are presented in the following sections. Then the promising channel sounding system based on software-defined radio (SDR) is discussed. The multi-antenna system combining with conventional channel sounder can be an enhanced sounding system that provides additional information in space. This system is also addressed in this chapter with initial measurement results. Finally, these channel sounding techniques still differ substantially in their approaches and have their own advantages and disadvantages, which are evaluated in the discussion of this chapter.

The measurement of propagation characteristics is a quite complex but crucial step in mobile radio system design and network planning. Accurate devices and rational approaches are essential to extract key parameters of a wireless channel that will provide efficient coverage and reliable data transmission in different scenarios at different frequencies. Channel sounding is a technique can be used for: (i) understanding the behavior of the radio channel; (ii) building more and more accurate model for the wireless channel; (iii) improving and validating the algorithms. However, for different measurement scenarios and purposes, the choice of channel sounding technique are usually different. The options include the narrowband and wideband transmissions, also whether a time domain or frequency domain characterization is required [54].

- Narrowband channel sounding: the unmodulated Continuous-wave carrier (single tone) is adopted in narrowband channel sounding technique to excite the channel, and the variations in power and phase are captured by a moving or a stationary receiver. For instance, the transmitter sends a series of single tone continuous sinusoidal waves with different carrier frequencies; then the waves are measured by the receiver and collected in the spectrum analyzer. With this experiment setup, the channel transfer function of the wireless channel can be obtained. However, this approach can not show the multipath effect by the spectral lines in a spectrum analyzer, when two frequencies are correlated. 
Chapter 3. Channel Measurement Systems

- wideband channel sounding: Since the narrowband sounding techniques are inefficient to capture the frequency and time coherence behavior of the wireless channel [55], the wideband channel parameters, such as the maximum delay spread, the RMS delay spread, and the coherence bandwidth are of particular importance to determine the small-scale effects, which can be obtained by the sounding signal occupying wide bandwidth. The typical wideband channel sounders are reviewed in detail in the following section.

\subsection{Channel Sounders}

According to the different measurement objectives and methods, the wideband sounder can be divided to two categories as follows: [56]:

- Channel sounder for frequency domain measurements

The most straightforward means of performing channel measurements in the frequency domain is directly estimate the transfer function. Sweptfrequency sounder ( or "Chirp Sounder") measures the complex frequency response of a channel by sweeping a single carrier across different frequencies in quick succession and comparing the amplitude and phase of the received signal at each frequency with the corresponding transmitted signal. These sweeping can measure different frequencies at different times. The transmit waveform covers the range $\Delta f$ of interest is represented by:

$$
p(t)=\exp \left[2 \pi j\left(f_{0} t+\Delta f \frac{t^{2}}{2 T_{\text {chirp }}}\right)\right] \quad 0 \leq t \leq T_{\text {chirp }}
$$

Then the instantaneous frequency is $f_{0}+\Delta f \frac{t}{T_{\text {chirp }}}$, which changes linearly with time. Besides, Sounding the channel on different frequencies at the same time is another option, i.e. the sounder generates different sinusoidal sounding signals at different frequencies with different amplitudes and phases, then transmit them simultaneously. The vector network analyzer(VNA) can be used as the swept-frequency stimulus-response test set and directly measure the complex frequency response of the channel. Then 
the inverse Fourier transform to the measured response can get the channel impulse response [2].

\section{- Channel sounder for time domain measurements}

Wideband channel sounding techniques for time-domain measurements are proposed mainly based on the periodic narrow pulse approach, and the pulse compression methods. Depending on different receiver structures, pulse compression is realized by matched filter or cross correlation [55]. For time domain measurements, the channel sounders are expected to obtain the impulse response of the channel directly. Generally, the transmitted signal $s(t)$ is given by:

$$
s(t)=\sum_{i=0}^{N-1} a_{i} p\left(t-i T_{R e p}\right)
$$

where $\mathrm{N}$ is the pulse numbers in the measurement, $a_{i}$ is the pulse amplitude of the $i^{t h}$ pulse, and $p(t)$ is the periodically repeated pulse in every fixed interval $T_{\text {Rep }}$. Based on the choice of transmit waveform $p(t)$, the channel sounder for time domain measurements is commonly divided into two types:

- The periodic pulse sounder/impulse sounder provides a quick and direct solution to observe the impulse response of a wireless channel as Fig. 3.1 illustrated. The repetitive short pulse waveform is treated as an approximation of impulse with a pulse width $T_{W}$ and an interval of $T_{R}$. Thus the corresponding bandwidth of the interest spectrum is $B W=2 / T_{W}$. On the receiver side, the received signal is firstly filtered by a bandpass filter with a bandwidth of $B W$. Then the signal is amplified, measured by an envelope detector to get the attenuation, and eventually displayed and stored on a high-speed digital oscilloscope. The proper synchronization method for this kind of sounder is of particular importance. It is possible to be synchronized by coaxial/fiberoptic cables for indoor environments. Whereas in the case of longer distance is set between the transmitter and receiver, such as outdoor scenarios, the Global Positioning System (GPS) could be a practical 
proposal, which can simultaneously record the location as well [57]. Alternatively, the rubidium clocks can be applied instead of GPS because of the high stability. Consequently, the averaged power delay profile can be obtained after the post-processing on the RAW data. So, each narrow pulse provides a "snapshot" of the multipath channel in each certain time interval, and the combination of a series of "snapshots" can be used to understand the channel behavior over the measurement duration.

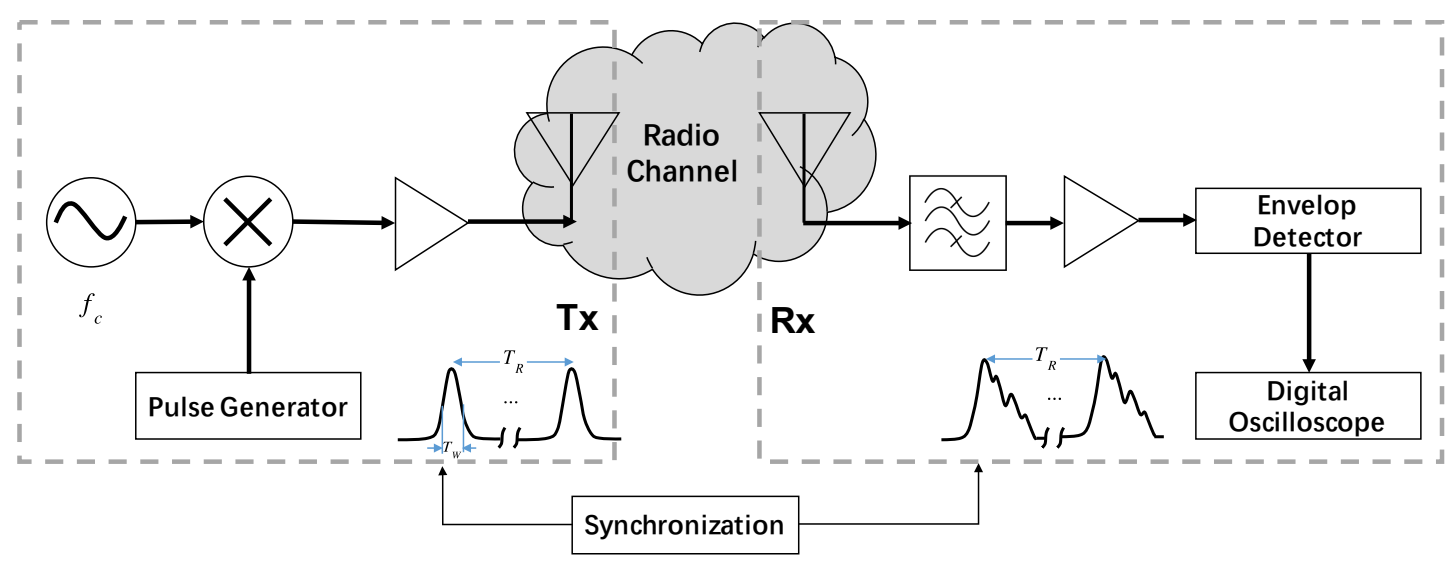

Figure 3.1. Principle of a periodic pulse sounder.

- The correlative sounder is based on the pulse compression for linear systems, which compresses the bandwidth of the wideband signal. Assuming the channel and transceiver are linear, the output $y(t)$ of the channel is the convolution of the pulse $p(t)$ and the impulse response $h(t)$ :

$$
y(t)=h(t) p(t)=\sum h[\tau] p[t-\tau]
$$

Commonly, the correlative sounder uses a pseudo-noise (PN) sequence as the input signal to probe the channel as shown in Fig. 3.2. The maximum-length PN sequence (m-sequence) is a finite-length sequence of periodic binary numbers (i.e. 0/1) generated using linear feedback shift registers (LFSR), which appears to be random as the noise. M- 
Chapter 3. Channel Measurement Systems

sequence is popular for wideband channel sounding, because it is easy to create, balanced, and having excellent autocorrelation and crosscorrelation properties.

There are two approaches to implement pulse compression technique: (i) Convolution Matched-Filter, mostly is a Surface Acoustic Wave (SAW) filter. It is a kind of asynchronous sounding, which is no need to re-generate the transmitted $m$-sequence at the receiver side. However the variances in manufacturing process of SAW filters and the requirement of fast recording equipment limit the performance of the SAW devices within the system. (ii) The Swept Time Delay CrossCorrelation (STDCC) is another realization of pulse compression technique at the receiver, which correlates an incoming signal with a copy of the transmitted m-sequence and clocked at a slightly lower sampling rate. The time dilation provides effective bandwidth compression, thus reduces the data capture and archival needs of the system. For $i \in\left\{1, \ldots, L_{m}\right\}$, let $b_{i} \in\{0,1\}$ and $p[i]=2 b_{i}-1 \in\{-1,1\}$ as the antipodal signal, the normalized autocorrelation function $R_{m}[k]$ at the receiver is given by [38]:

$$
R_{m}[k]=\frac{1}{L_{m}} \sum_{i=1}^{L_{m}} p[i] p[i+k]=\left\{\begin{array}{rlrl}
1 & k & =0 \\
-\frac{1}{L_{m}} & k & \in\left\{1, \ldots, L_{m}\right\}
\end{array}\right.
$$

Combining Eq. 3.3 and Eq. 3.4, the impulse response can be obtained as the output of the correlator in Fig. 3.2 [38]. In other words, the received complex baseband signal is multiplied by a replicate of the transmitted m-sequence and low-pass filtered. The STDCC-based correlative sounder is also known as the "sliding correlator sounder".

VNA as the channel sounder for frequency domain measurements in various environments can be found in many publications, such as on-board a warship at 2 and $5 \mathrm{GHz}$ [58], in an underground mine at $2.4 \mathrm{GHz}$ [59], inside a rectangular metal cavity from $3 \mathrm{GHz}$ to $10 \mathrm{GHz}$ [60]. Temporal distributions of multipath delay with the associated power have been early measured in New York City at $450 \mathrm{MHz}$ by the periodic pulse sounding method [61], then the measurements with 
Chapter 3. Channel Measurement Systems

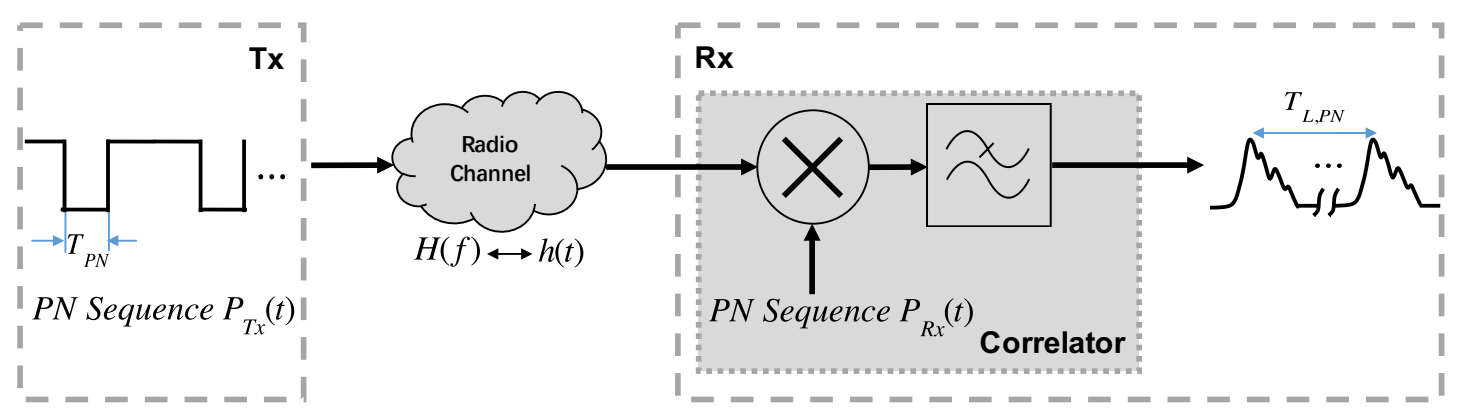

Figure 3.2. Principle of a correlative channel sounder.

a narrower pulse (50 ns) at higher frequencies $(2.9 \mathrm{GHz})$ have been conducted in [62] and [63], respectively. Besides the urban scenarios, this technique has also been employed for indoor environments [32] [64] [65].The sliding correlator sounder has been firstly used to measure channel impulse responses in outdoor suburban environments at $910 \mathrm{MHz}$ [66], in the office buildings at $850 \mathrm{MHz}, 1.7$ $\mathrm{GHz}$ and $4 \mathrm{GHz}$ [67], and in a large train yard at $915 \mathrm{MHz}$ [68].

In the past decades, a series of wideband channel sounder has been launched. For instance, wideband sounder at $2.4 \mathrm{GHz}$ [69], wideband MIMO sounder at $5.8 \mathrm{GHz}$ [70], a parallel receiver channel sounder for spatial and MIMO characterization [71], and a VNA-based MIMO channel sounder has been proposed for indoor measurements at $60 \mathrm{GHz}$ [72]. The commercial sounder systems are also of interest and have been employed in a number of measurements, such as the SIMOCS 2000 produced by Siemens [73], ECHO 24 channel sounder developed by the Swiss Federal Institute of Technology Zurich [74], the RUSK sounders family manufactured by MEDAV GmbH [75] [76], and the Elektrobit Propsound CS ${ }^{\mathrm{TM}}$ MIMO channel sounder [77];

\subsection{Channel Sounder Development}

\subsubsection{Requirements}

For channel sounder development, the following general requirements summarized in [57] must be fulfilled to make the sounding signal perform efficient measurements: 
Chapter 3. Channel Measurement Systems

- Large bandwidth: since the minimum resolvable delay between multipath components is equal to the probing pulse width. Thus the impulse must be sufficiently narrow to ensure that the signal bandwidth is larger than the channel coherence bandwidth to obtain a considerable resolution and capture as much echoes as possible.

- Large time-bandwidth product (TBP): for instance, a longer m-sequence means a longer period and bigger TBP (because the bandwidth is constant). Thus a higher transmission power is allowed, which results in a higher SNR and smaller error rate at the receiver.

- Signal duration: On the one hand, a larger TBP is obtained by a longer signal duration. On the other hand, the signal duration should not be longer than the coherence time to maintain the channel's stationary. Therefore, the ideal signal duration is larger than the maximum excess delay of the channel but shorter than the coherence time.

- Power-spectral density (PSD): the PSD of the sounding signal $\left|P_{T X}(j w)\right|^{2}$ should be uniform over the bandwidth of interest to ensure the same quality of channel estimation inside this bandwidth, and little signal energy outside the bandwidth to yield higher efficiency.

- Low peak-to-average ratio (PAR): A lower PAR $\left(C=\frac{|p(t)|_{\text {peak }}}{p(t)_{r m s}}\right)$ implies efficient transmit power amplifier.

- Good correlation properties: For correlative channel sounder development, the autocorrelation function (ACF) should be set with a high peak off peak ratio (POP), and a zero mean allows unbiased estimates.

Generally, for wideband channel sounder design or optimization, the following aspects should be involved: (i) a rational pulse width to meet the requirement of measurement resolution. (ii) a compromised sounding signal duration according to the channel coherence time and the required TBP. (iii) the same absolute value for all frequency components inside the bandwidth of interest to obtain a constant PSD. (iv) adjusting the phases of the frequency components to obtain a low PAR. 


\subsubsection{Self-developed Channel Sounders}

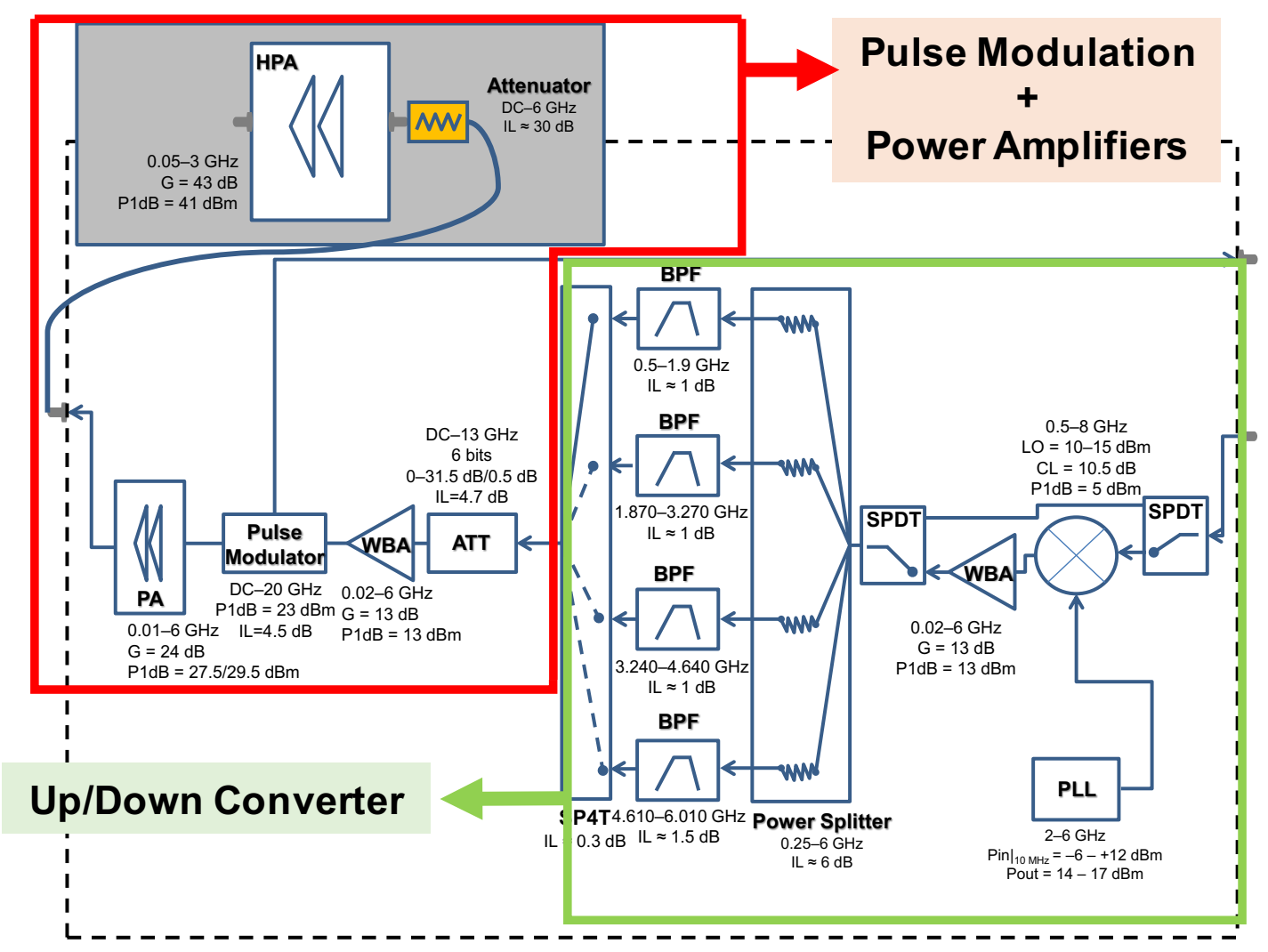

Figure 3.3. High-ended channel sounder transmitter front-end.

High-end Channel Sounder On the basis of periodic pulse sounding approach, a channel sounder system has been designed at Technical University of Madrid (UPM) and tested in real railway environment [78]. This system is a flexible and extensible hardware that covers multiple frequency bands include LTE/LTE-A bands. Thus, it can be applied as the RF front-end for LTE RF link measurements, and also can work individually for channel sounding measurements with high performance.

The transmiting system is composed of two subsystems: the baseband subsystem and RF subsystem. The baseband signal can be generated by a computer and an FPGA, for instance, a USRP configured by the host computer can generate the LTE baseband signal for LTE propagation testing. Then the baseband signal is delivered to the RF front-end subsystem as illustrated in Fig. 3.3. It is 
Chapter 3. Channel Measurement Systems

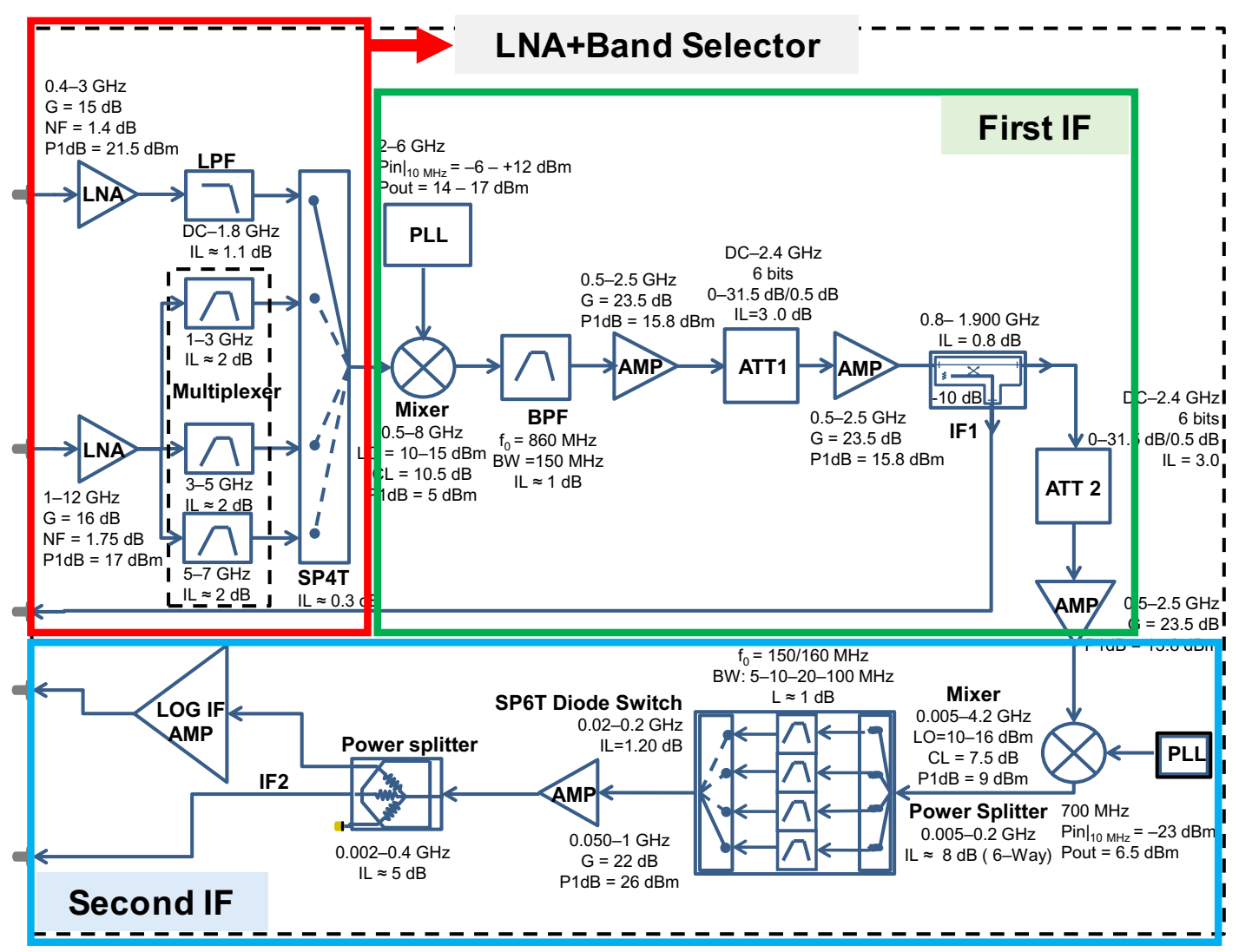

Figure 3.4. elf-developed channel sounder receiver front-end.

comprised of two main parts:

- Up/Down converter: the RF transmitter front end receives the modulated signal from the baseband system, removes the unwanted signal and noise, then converts it to the output signal at desired frequency. For this purpose, the baseband signal is directly injected into the front-end, or mixed with a wideband frequency synthesizer (HMC-C083) covered 2-6 GHz, then up/down converted by a 4-bands switch filter bank for selecting the desired frequency band up to $6 \mathrm{GHz}$. Also, the local oscillator (LO) leakage and harmonics are rejected by the bandpass filters (BPFs).

- Pulse modulation \& Power amplifiers: the first stage of this unit is the optional process of the pulse modulation that controlled by an SPST switch. The pulse modulator is applied to generate the narrow pulse sounding sig- 
nal. The other option is inactivating the pulse modulator and offering the signal tone in CW mode. The final stage is the power amplifier unites. The RF subsystem provides a $29.5 \mathrm{dBm}$ internal power amplifier (PA), and an external high power amplifier (HPA) for higher amplification requirement up to $42 \mathrm{dBm}$.

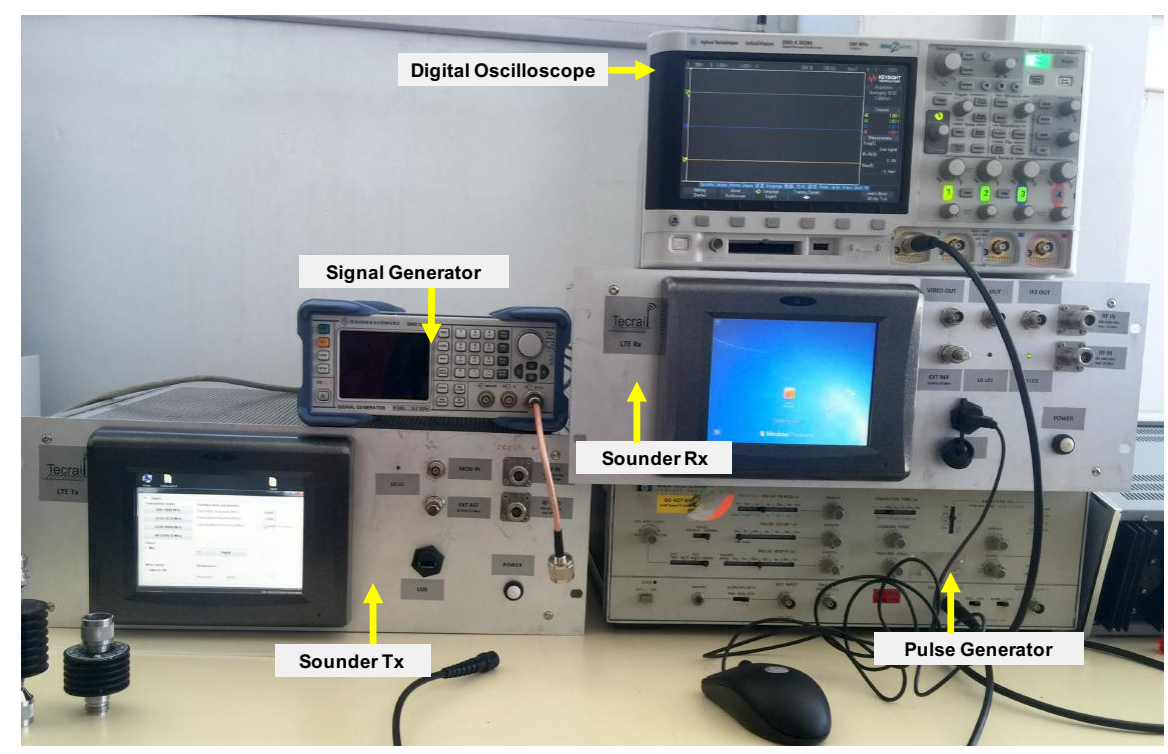

Figure 3.5. Photograph of the channel sounder system.

Correspondingly, the receiving system is in charge of converting received signal at the high frequency to an intermediate frequency (IF) for better observation, and recording the channel impulse response to analyzing the channel behavior. The receiver front-end is designed to a dual-conversion structure and composed of three units as Fig. 3.4 demonstrates:

- Low noise amplifier (LNA) and filter bank: the received signal is amplified and filtered out the image frequency and adjacent band interferences in this unit. Two LNA are employed to reduce the noise figure and increase the dynamic range of the system at two frequency bands: $0.4-3 \mathrm{GHz}$ and 1-12 GHz, respectively. Then the filter bank provides four bands cover the LTE/LTE-A bands for different applications.

- The first IF stage (IF1): after the filter bank, a wideband mixer is followed by an IF $850 / 860 \mathrm{MHz}$ filter. Thus the filtered signal is mixed with a 


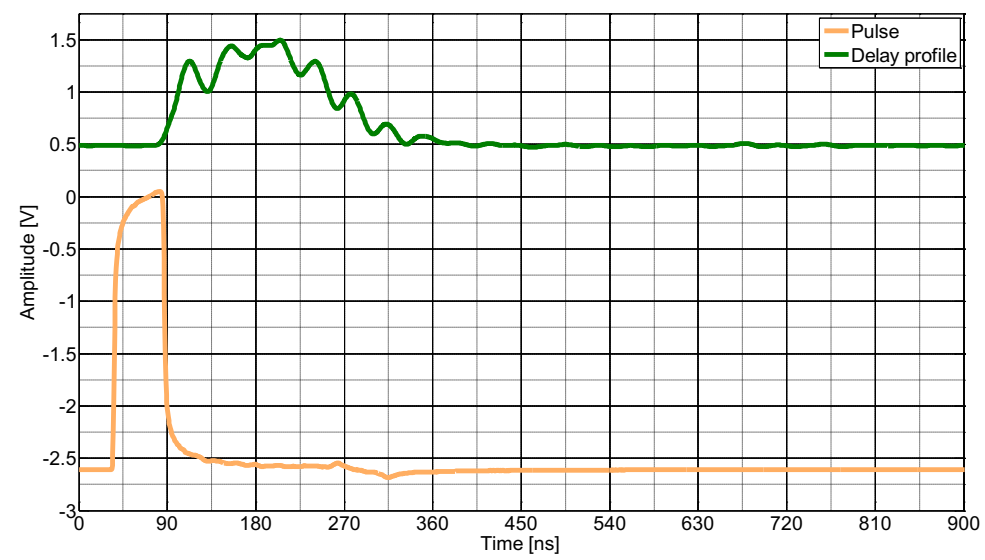

Figure 3.6. Single pulse synchronization.

frequency synthesizer(HMC-C083) covering from 2-6 GHz, then obtain the first IF signal at 850/860 MHz. This IF signal passes amplifiers and a programmable attenuator and provides the output at the constant power level. The final IF1 signal can be observed after a 10-dB directional coupler.

- The second IF stage (IF): the other branch of IF1 then arrives the second IF stage. Firstly, it is mixed with a fixed $700 \mathrm{MHz}$ PLL by a narrowband mixer, and the $150 / 160 \mathrm{MHz}$ output is obtained. Then another four filter bank is used to match the channel bandwidth. This filter bank provides $5 / 10 / 20 / 100 \mathrm{MHz}$ as options. Eventually, a proper logarithmic detector is set at the end of one branch. The logarithmic detector is used to demodulate the received pulse, and an $\mathrm{RF}$ power meter is set to measure the other branch to provide the power information in the channel sounder operation mode.

Besides the RF front-ends, the complete high-end channel sounder has to include: the signal generator to generating the baseband signal, the pulse generator to produce the narrow pulse trains, a digital oscilloscope to record and pre-process the signal captured at the receiver side, and the corresponding antennas for both transmitting and receiving systems. An example suite of the channel sounder equipment is shown in Fig. 3.5. For synchronization, Fig. 3.6 shows an example of the single transmitted pulse and the corresponding delay profile at the receiver. 
Chapter 3. Channel Measurement Systems

Table 3.1. High-end channel sounder profiles.

\begin{tabular}{l|c}
\hline \hline \multicolumn{2}{c}{ Transmitter } \\
\hline Frequency Range & 4 bands: $0.5-1.9 / 1.9-3.2 / 3.2-4.6 / 4.6-6.0 \mathrm{GHz}$ \\
\hline Output Power & up to $42 \mathrm{dBm}$ \\
\hline Modulation & Pulse: up to $10 \mathrm{~ns} /$ External: LTE \\
\hline \multicolumn{2}{c}{ Receiver } \\
\hline Frequency Range & 4 bands: $0.4-1.8 / 1.0-3.0 / 3.0-5.0 / 5.0-7.0 \mathrm{GHz}$ \\
\hline IF Dual Conversion & IF1: $860 / \mathrm{IF} 2: 160 \mathrm{MHz}$ \\
\hline IF Bandwidth & $5 / 10 / 20 / 100 \mathrm{MHz}$ \\
\hline Noise Figure & $4 \mathrm{~dB}$ \\
\hline Demodulation & Logarithmic Detector / LTE Demodulator \\
\hline Dynamic Range & $90 \mathrm{~dB}$ \\
\hline \hline
\end{tabular}

The synchronization process is conducted by the single pulse. Furthermore, the technical parameters of the channel sounder system are listed in Table. 3.1.

\section{Portable Channel Sounder}

Although the high-end channel sounder above provides excellent performance and precise experiment results, the size and weight of this sounder with assorted equipment and peripherals eliminate the portability and convenience during the actual measurement. To improve the mobility of the channel sounder system, we have designed a portable channel sounder. Unlike the bulky channel sounder above, the portable channel sounder is highly integrated and easy to configure.

For the portable channel sounder transmitter, the Trinity Power Incorporated (TPI) synthesizer (version 4.1) is adopted to replace the external signal generator. The TPI synthesizer covers from $35 \mathrm{MHz}$ to $4.4 \mathrm{GHz}$, and can be controlled and powered by a laptop computer running Windows via its USB port. The customized software on a laptop computer can generate the PLL synthesized RF signal at the desired frequency.

Then, the DS1040-75 programmable one-shot pulse generator is employed as the alternative solution of the conventional external pulse generator. This pulse generator is only $0.001 \mathrm{~kg}$ at an extraordinarily small size. Five programmable pulse widths (15/30/45/60/75 ns) are available for easy selection. 
As shown in Fig. 3.7, the signal at desired frequency is generated by the synthesizer and then modulated by pulse modulator to get a narrow pulse with selectable pulse widths. The SPST switch is used to set the transmitter working in pulse mode or continues wave mode for different channel sounding purposes. In the final stage of the transmitter, the sounding signal is amplified by a high power amplifier and sent to the wireless environment. Also, the transmitter offers a TTL trigger output port for synchronization usage.

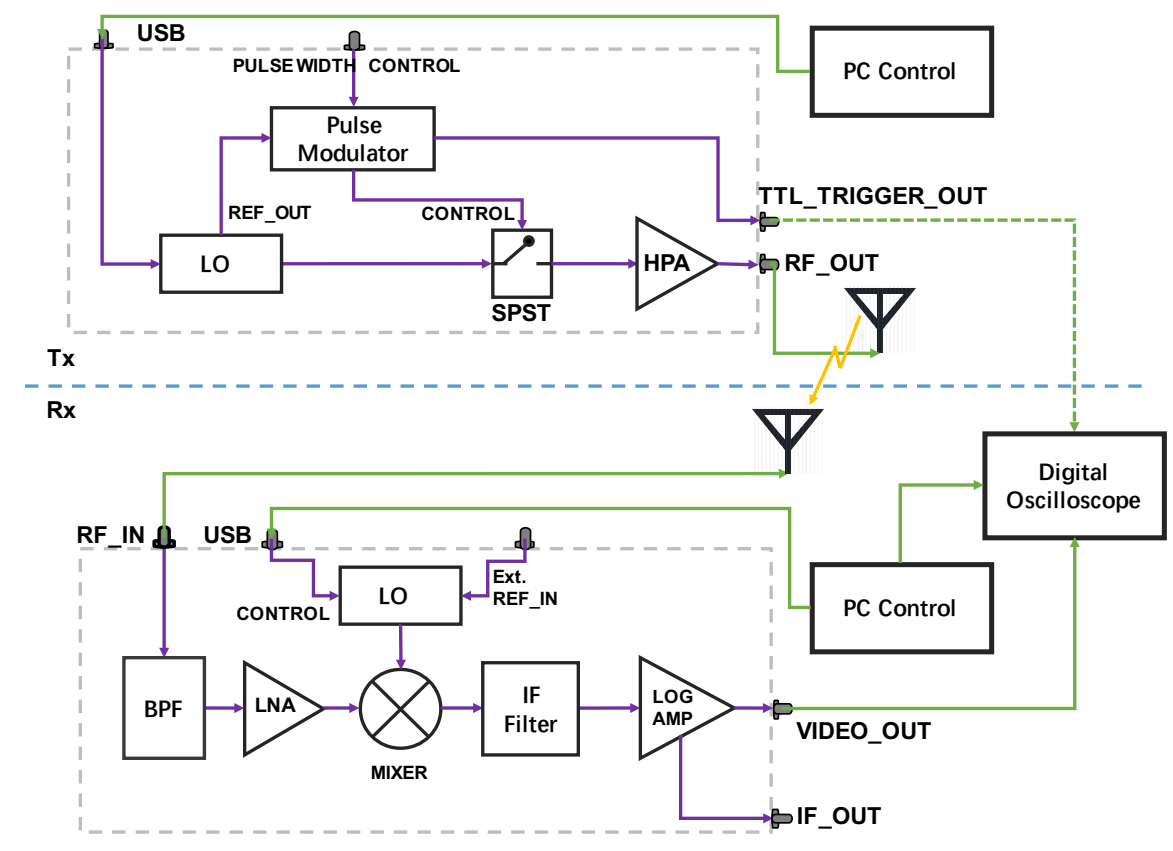

Figure 3.7. Block diagram of the portable channel sounder.

At the receiving end, the radio signal is captured by the receiving antenna, filtered, and sent to the LNA. Then, the amplified signal is mixed with a local oscillator signal (also generated by the TPI synthesizer) for superhet receiving to obtain a $+/-150 \mathrm{MHz}$ intermediate frequency, through an addition or subtraction to the transmitted signal frequency. A $100 \mathrm{MHz}$ band pass filter is followed to remove the unwanted parts. Next, the logarithmic amplifier is used as an envelop detector, which can provide the magnitude of the received signal. Eventually, the digital oscilloscope displays the PDP and stores the raw measurement data for post-processing. 
Chapter 3. Channel Measurement Systems

Table 3.2. Portable channel sounder profiles

\begin{tabular}{l|c|l|c}
\hline \hline \multicolumn{2}{c|}{ Transmitter } & \multicolumn{2}{c}{ Receiver } \\
\hline Frequency Range & $35-3000 \mathrm{MHz}$ & Frequency Range & $100-4550 \mathrm{MHz}$ \\
\hline Output Power & up to $36 \mathrm{dBm}$ & IF / IF Bandwidth & $150 / 100 \mathrm{MHz}$ \\
\hline Pulse Width & $15 / 30 / 45 / 60 / 75 \mathrm{~ns}$ & Noise Figure & $3 \mathrm{~dB}$ \\
\hline Pulse Period & $1000 \mathrm{~ns}$ & Max. Input Power & $-20 \mathrm{dBm}$ \\
\hline Reference Frequency & Internal: $10 \mathrm{MHz}$ & Sensitivity & $-100 \mathrm{dBm}$ \\
\hline Power Supply: $12 \mathrm{~V}, 5 \mathrm{~A}$
\end{tabular}

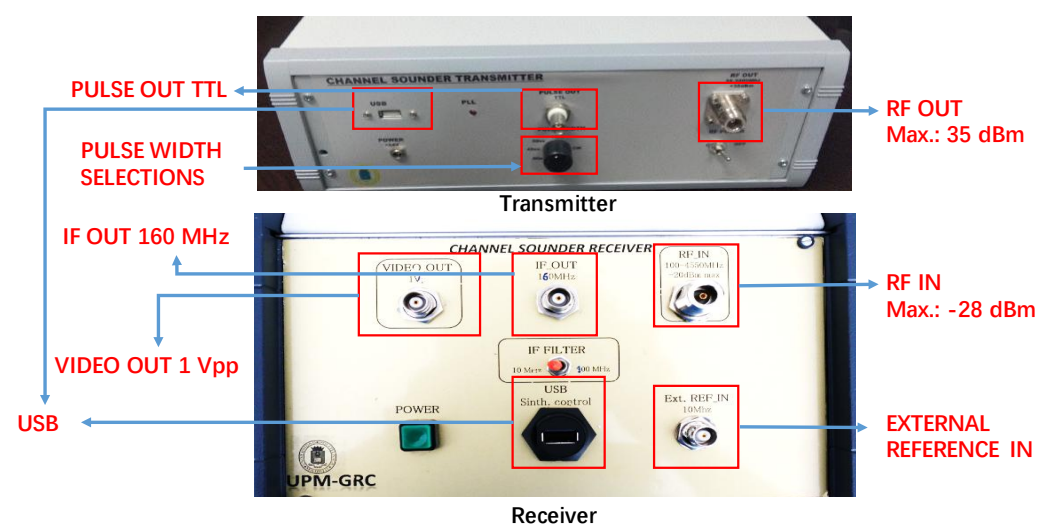

Figure 3.8. Protograph of the portable channel sounder

The specific parameters of transmitter and receiver can be found in Table. 3.2. The photograph of the portable channel sounder is presented in Fig. 3.8. The overall system is less than $10 \mathrm{~kg}$, which is easy to implement by the portable hardware coupled with customized software.

\subsubsection{SDR-based Channel Sounder}

The Universal Software Radio Peripheral (USRP) is a low-cost hardware, which is commonly used to realize a typical software defined radio platform together with radio software, e.g. the open-source software GNU Radio in Linux and the shared software Labview in Windows. With easily configurable parameters such as the center frequency, bandwidth (sample rate), gain, etc., it is a promising system for the data collection in complex environments. With the assistance of USRP, 
Chapter 3. Channel Measurement Systems

the simpler and quicker channel measurements are expected to be conducted at modest cost, thus reducing the cycle time for further channel modeling, design and deployment efforts. Note this approach obtains the advancement of the rate by sacrificing some level of accuracy and sensitivity, compared to the conventional channel measurement campaigns using laboratory equipment or custom-built device [79]. The SDR-based sliding correlator channel sounding systems have been proposed and carried out some initial propagation measurements. For instance, the USRP and PN sequence based channel sounding with $4 \mathrm{M}$ chips/s [80]; the sliding correlator achieved 60 ns temporal resolution has been implemented for wideband measurements in indoor and outdoor enviroments [79]; the spread spectrum channel sounder experiments on USRP N210 [81]; the simulated sliding correlator in Labview has been designed for comparison of PN sequence lengths [82]; the delay and Doppler spread have been parameterized by the SDR-based channel sounder in different scenarios [83].

\section{Passive Sounding System}

Besides the sliding correlator method, another inexpensive and easy-to-use methods for measuring the channel is the so-called passive sounding system [84, 85]. Namely, the passive sounding measurement only needs the dedicated receivers to sounding the existing signals. Thus the active procedure of transmitting the sounding signals can be omitted. The main idea is to exploit the downlink signals of the commercial in-service communication networks e.g. Universal Mobile Telecommunications System (UMTS) networks and LTE networks. When the transmitted signals in the commercial network as the sounding signals are known, the downlink channel impulse responses or the channel transfer functions can be obtained with valuable channel information.

Specifically, the common pilot channel of UMTS networks is transmitted with known bit sequences (m-sequence), and the cell-specific reference signal of LTE networks is transmitted on specific subcarriers mapping to known complex-valued sequences. A direct and economic approach is to record the real-time downlink signals and then calculate the CIRs offline due to the fact that the real-time downlink signals can be easily collected by using a Universal Software Radio 
Chapter 3. Channel Measurement Systems

Peripheral (USRP) device controlled by a host computer. Therefore, the measurement system can be simply composed of a USRP device, a host computer with SDR software installed, and receiving antenna(s).

The recorded downlink raw data can be processed in four steps:

- Filtering: in this step, a rectangle window in the frequency domain with the same width of UMTS signal bandwidth is applied to remove possible interferences in the neighboring frequency range (the sample rate is usually larger than Nyquist sample rate) and to compress the noise power.

- Synchronization: the beginnings of the time slot and the time frame and the bit sequence possibilities (8 possible bit sequences) used in the CPICH are determined by matching the primary synchronization code and the second synchronization code with the received data respectively.

- Scrambling code detection: the exactly used bit sequence of the CPICH is determined by matching all the eight possibilities with the received data.

- CIR extraction: CIRs are extracted by the correlation of the received data in the CPICH frame (other channels included can be neglect since the UMTS is a code division multiple access (CDMA) system) with the transmitted $\mathrm{CPICH}$ bit sequence determined in the former steps. The CIRs are represented by the ACF of the bit sequence, which is mathematically the same with the correlative sounder. Also, the bandwidth of the bit sequence is $3.84 \mathrm{MHz}$.

Compared to the UMTS, LTE systems have a higher effective bandwidth (up to $18 \mathrm{MHz}$ ), which results in higher delay and spatial resolution. The offline processing for the LTE networks is very similar. The real-time downlink data is also first filtered to remove interferences in the neighboring frequency range and to compress the noise power. After that, the primary synchronization signals (PSSs) and secondary synchronization signals (SSSs) are detected with their locations and indices determined. Also, the cyclic prefix (CP) type (normal or extended) can be known by examining the distance between the PSS and SSS in the time domain. The information of PSS, SSS, and CP together determined the transmitted cell-specific reference signals (CRSs) in the time and frequency domain, 
Chapter 3. Channel Measurement Systems

i.e., the occupied symbols and subcarriers of CRSs are obtained. By the inverse Fourier transformation in the last step, the CIRs can be easily acquired form the channel transfer functions.

\subsubsection{Multi-antenna system}

MIMO system points to a promising solution to enhance channel capacity and improve QoS. The evaluation of the effectiveness of these multiple antenna systems in the radio environment is of interest. The additional aspects of the radio channel can be characterized by the antenna arrays with multiple transmitting and receiving antennas in conjunction with suitable signal processing or data reduction methods. The distribution of angles at which signals leave the transmitter and/or arrive at the receiver can be extracted in this way. Consequently, the correlation between the fading signals observed at each antenna element in spatial diversity and MIMO transmission schemes can be obtained. A double-directional channel model needs a full characterization of MIMO channels by estimating the angle-of-arrival (AoA) distribution observed at the receiver side for rays launched at each of the possible angles-of-departure (AoD) from the transmitter side [2].

The simplest multi-antenna system involves using fixed antennas at the transmitter and/or receiver. For example, mounting a single antenna on a mechanical device and moving it through a set of closely spaced positions in space. Then the virtual array can be generated and thereby spatial correlation, temporal correlation, and angle-of-arrival distributions can be characterized. Alternatively, we mouth 6 APA-M25 panel antennas on a multi-ports RF circle switch (EMIEYAL LR-3930-XO-21-41), which is controlled by a USB I/O controller as shown in Fig. 3.9. Each element in this hexagon antennas array can be activated by the preconfigured RF switch port within a certain period. With a complete sweeping of 6 antennas, the transmitted signals can be individually received from every $60^{\circ}$ in space.

An example measurement has been conducted with this multi-antenna system in a subway train. The high-ended channel sounder is implemented in conjunction with the antenna array. The narrow pulse is transmitted by a single antenna at the transmitter side, and received by the antenna array at different AOA as shown 


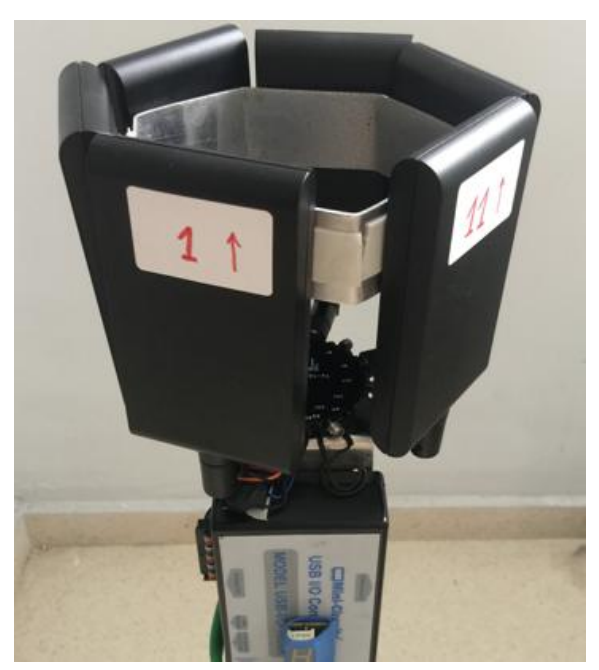

Figure 3.9. Photograph of the hexagon multi-antenna system.

in Fig. 3.10.
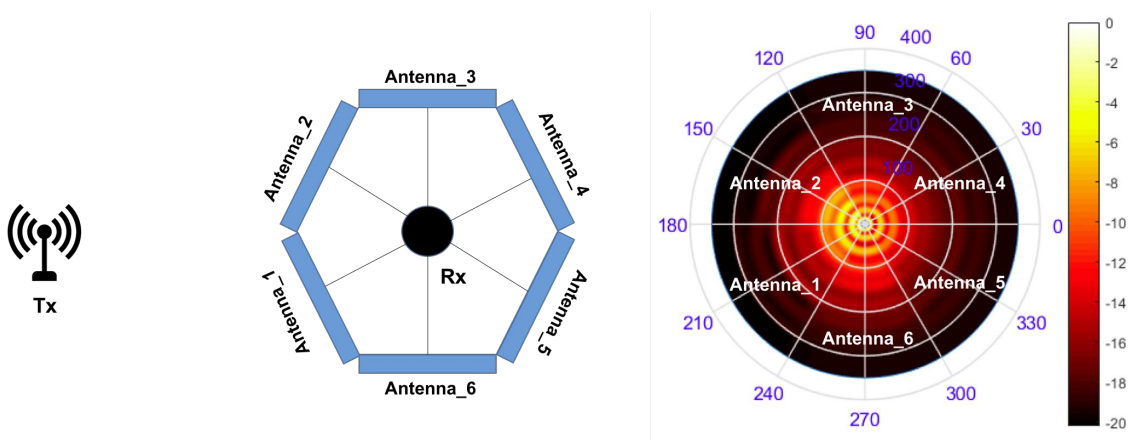

Figure 3.10. Channel sounding with multi-antenna system.

The averaged PDPs at the receiver side are distributed with the corresponding antennas and shown in Fig. 3.10. The results perform significant directional dependence. The receiving antennas faced to the transmitter appears much stronger power in the LOS path, whereas the rest PDPs from the other directions shows larger fluctuations and more complex multi-path components, especially at the backside, e.g. Antenna 6. These measurement results are rational and of interest for future MIMO channel measurements and modeling. 


\subsection{Discussions}

This chapter first reviews the channel sounding techniques for narrowband and wideband measurement. The single-tone $\mathrm{CW}$ system can be employed to measure the path loss and fading statistics over the radio link with simple and cheap hardware system. For exploiting the multipath propagation in complex environments, the wideband channel sounder system has to be employed for analyzing the time delay spread and spatial information. This chapter summarizes three common wideband channel sounder for the time-domain and frequency-domain measurements.

For swept-frequency sounders, VNA is usually implemented to measure the channel responses over short ranges (e.g. indoor environments). It is able to track system error and provide an absolute delay to power delay profile, and also a relatively flexible system easy to apply. But there are some drawbacks can not be ignored: (i) the VNA are generally not portable and expensive; (ii) the individual frequency sweep with a relatively slow collecting rate; (iii) it is difficult to ensure the channel is relatively stationary in each frequency sweep time; (iv) the synchronization between the transmitter and receiver over ranges greater than a few hundred meters requires extra work.

The advantages of this periodic pulse sounding are good time resolution and the ability of real-time processing. However, only the amplitude variations are recorded by the envelope detector, whereas phase information is discarded. The coherent detector may overcome this problem and measurement the multipath phases. Theoretically, the periodic pulse sounding technique is an ideal technique, if the pulse duration approaches zero since the delay resolution is infinitely small [55]. However, this system is subject to interference and noise, due to the wide passband filter required for multipath time resolution. Therefor the periodic pulse channel sounder requires a transmit power amplifier with a large dynamic range in order to guarantee the accurate measurements. Moreover, the synchronization of this system is used to relying on the ability to trigger the oscilloscope on the first arriving signal. If the first arriving signal is blocked or fades, the system may not trigger properly.

One of the biggest advantages of correlative channel sounder, compared to the 
periodic pulse sounder, is the ability to reject passband noise and thus improving the coverage range for a given transmitter power. PN sequence synchronization between the transmitter and receiver is eliminated by the sliding correlator. Also, sensitivity is adjustable by changing the sliding factor and the post-correlator filter bandwidth. Hence, required transmitter powers can be considerably lower than comparable direct pulse systems due to the inherent "processing gain" of spread spectrum systems. However, it is difficult to measure the channel in real time. Since the PN codes sliding past each other, the measurement time is long due to the extra processing time of pulse compression. The phases of individual MPCs can not be measured by a coherent detector. Even if coherent detection is used, the sweep time of a spread spectrum signal induces delay such that the phases of individual MPCs with different time delays would be measured at substantially different times, during which the channel might change [48].

For the passive sounding system, the lack of knowledge of the transmitted signal may obstruct the channel modeling. For instance, the absolute propagation path delays can not be obtained, since the time information at the transmitting sides is unknown. Another drawback is that frequencies are fixed in the communication networks, which confines the channel investigation in several frequency bands. Nevertheless, the SDR-based passive sounding measurement can be conducted extensively and conveniently since the wide coverage of commercial communication networks. Also, the light weight and the simpleness of the SDR-based sounding hardware (e.g. USRP) result in the efficient work in multiple scenarios, e.g. HSR scenarios, metro scenarios, LAFO scenarios. With these advantages, channel measurements based on SDR starts to attract more attentions. 


\section{Chapter 4}

\section{Propagation in Underground Environments}

The wireless communication network in underground environments is one of the promising application areas of the recently developed wireless networking techniques. The underground wireless communication network consists of wireless devices that work below the ground surface. These wireless devices can be classified depending on two main applying directions [86].:

- Wireless Underground Sensor Networks (WUSNs), the wireless nodes are completely buried under dense soil, rock, or water, as the new communication medium with respect to the air. Significant attention to WUSNs capabilities for monitoring various underground conditions is caused by the variety of existing and potential applications. For instance, intelligent irrigation, environmental monitoring, infrastructure monitoring, localization, and border patrol.

- Wireless communication links for underground spaces, the existing underground system such as mines, stations, road/subway tunnels are focused as the propagation environments for radio signals. In this case, the communication medium is the air in the bounded open underground space. It is essential to improve the safety and productivity in underground mines and to realize convenient and efficient communication links for drivers and passengers in road/subway tunnels and subway stations. 
The primary challenge for propagation in underground environments is the realization of efficient and reliable underground wireless links. For WUSNs, a wireless network with multiple hops and disseminate data for seamless operation has to be established in soil, rock, or water. Although the electromagnetic waves for terrestrial signal propagation have been widely investigated, the channel model in the underground medium needs a further study. Besides EM waves, Magnetic Induction (MI)-based WUSNs can be an alternative solution, which makes use of magnetic antennas implemented as coils, improves the transmission range due to a much lower path loss [87]. For the wireless communication system designed for underground mines/stations and road/subway tunnels, the EM waves are still the first choice for wireless signal propagates through the air. However, the propagation characteristics of EM waves are significantly different with respect to the terrestrial wireless channels, because of the enclosed spaces and the lossy dielectric walls/ceilings in the underground mines/stations or road/subway tunnels.

There have been several studies on analyzing the propagation of UHF and SHF radio signals in underground environments in the last decades. For instance, a hybrid tunnel propagation model has proposed based on the measurements at $900 \mathrm{MHz}$ on horizontal and vertical polarization in a typical coal mine [88]; the radio propagation model for underground low-power sensor nodes [89]; the measurements in road tunnel [90], railway tunnel [91], subway tunnel [92], etc. There are also few propagation characterizations based on wideband radio channel measurements, which have been made in a highway road tunnel at $5.2 \mathrm{GHz}[90]$; in the underground mining environments in the range of $150-900 \mathrm{MHz}$ [93], at 1 $\mathrm{GHz}[94]$, and at 2-5 GHz [95, 96].

The underground space becomes the inevitable choice of city development as potential and abundant resources. Since the urban transportation is under lots of pressure, improving the efficiency and capacity of the subway system are the urgent requirements. A large number of studies on ITS applications have been conducted $[97,98,99,100]$ to enhance operational safety and efficiency. Besides, various public wireless communication technologies have been incorporated in train signaling and control communication systems. Therefore, propagation characteristics at three most commonly used frequency bands: $900 \mathrm{MHz}, 2.4 / 5.8$ 
4. Propagation in Underground Environments

$\mathrm{GHz}$, are of high interest for both industry and academia of ITS.

This chapter presents both narrowband and wideband channel measurements and modeling in underground subway systems in Part I and Part II, respectively.

\subsection{Part I: Narrowband Measurements in Curved Tunnels}

In recent years, many research and development work on wireless propagation in various subway tunnels have been done. For instance, narrowband measurements have been conducted in circular tunnels at $450 \mathrm{MHz}$ and $900 \mathrm{MHz}$ [101]; in arched railway tunnels at $400 \mathrm{MHz}$ [102] and at $900 \mathrm{MHz}$ [91]; and in subway tunnels at $2.4 \mathrm{GHz}$ [92]. Then the measurement-based empirical model [103] and stochastic models have been proposed, such as two-slope models [104, 105], three-slope model [106], four-slope models [102, 107], five-zone models [108, 109, 110], etc. Other modeling methods also have been applied in channel characterization in tunnels, such as the modal analysis based on waveguide theory [111], GO approach [112, 113], and Vector Parabolic Equation (VPE) techniques [114]. These research work provide the understanding of wave propagation in tunnels, experimentally or deterministically. Most of these studies focus on the straight tunnels, whereas curved tunnels are rarely concerned.

Some simulations for the radio wave propagation have been applied in curved tunnels. For instance, theoretical deduction of modal analysis in [115]; ray tracing-like approaches with some specific cross sections in $[4,116,117,118,119$, 120, 121, 122]; the parabolic equation (PE) along with the alternate direction implicit (ADI) method in [123, 124]; hybrid modeling approaches in [125, 126]. These simulations approaches can somehow predict the propagation losses or attenuations. Yet, propagation characterization in curved tunnel merely by simulation is limited and insufficient. Curved subway tunnels with different cross section sizes and dimensions, wall roughness and materials, and floor structure from the road, railway, and mine tunnels can not be simply reproduced by the simulators. Hence, corresponding measurements are essential for further channel modeling in curved tunnels. 
This section presents a series of narrowband propagation measurements in various subway tunnels with different configurations at $900 \mathrm{MHz}, 2400 \mathrm{MHz}$, and $5800 \mathrm{MHz}$. The large-scale fading is characterized by the mean of measurements, which can be used for network planning, channel modeling, key techniques evaluation in underground tunnel environments at various frequencies.

\subsubsection{Measurement Scenarios and Configurations}

The two most common tunnels in subway systems are the semicircular tunnel and the other one with an arched cross section. Most of the arched tunnel have been built in the $80 \mathrm{~s}$ and $90 \mathrm{~s}$ of last century. Whereas the semicircular tunnels have been excavated by Tunnel Boring Machine (TBM), thus it is relatively new and commonly adopted in the new lines. The curvature radius range in subway tunnels is typically from $90 \mathrm{~m}$ to $1000 \mathrm{~m}$, and the slope of the tunnels is generally less than 5.5\%. In our case, the measurements are conducted in two types of curved tunnels in the subway system of Madrid. As Fig. 4.1 shows, arched tunnel consists of three plane walls and an arched roof; semicircular tunnel includes a semicircle roof and a plane floor, or an inclined plane in the case of the curved tunnel. The test system setup and relative position along the tracks inside the arched tunnel with dimensions $7.59 \mathrm{~m} \times 5.52 \mathrm{~m}$ and semicircular tunnel with $8.41 \mathrm{~m} \times 6.87 \mathrm{~m}$ are also illustrated in Fig. 4.1.

Table 4.1. Datasheet of the test systems for the narrowband measurements in subway tunnels.

\begin{tabular}{c|c|c|c|c|c}
\hline \hline Frequency & Output Power & Model & Type & Gain & Beam Width \\
\hline \multicolumn{2}{c|}{ Transmitter } & \multicolumn{5}{c}{ Tx Antenna } \\
\hline $920 \mathrm{MHz}$ & $27 \mathrm{dBm}$ & HG908 & Panel & $8 \mathrm{dBi}$ & $\mathrm{H}: 75^{\circ}, \mathrm{V}: 65^{\circ}$ \\
\hline $2400 \mathrm{MHz}$ & $24 \mathrm{dBm}$ & HG2414P & Panel & $14 \mathrm{dBi}$ & $\mathrm{H}: 30^{\circ}, \mathrm{V}: 30^{\circ}$ \\
\hline $5705 \mathrm{MHz}$ & $22 \mathrm{dBm}$ & HG5419P & Panel & $19 \mathrm{dBi}$ & $\mathrm{H}: 16^{\circ}, \mathrm{V}: 16^{\circ}$ \\
\hline \multicolumn{2}{c}{ Receiver } & & Rx Antenna \\
\hline $920-5705 \mathrm{MHz}$ & - & HL050 & Log-Periodic & $8.5 \mathrm{dBi}$ & $\mathrm{H}: 85^{\circ}, \mathrm{V}: 70^{\circ}$ \\
\hline \hline
\end{tabular}

For the purpose of conducting narrowband measurements, the transmitting system consists of a continuous wave $(\mathrm{CW})$ transmitter $(\mathrm{Tx})$ and a directional 


\section{Propagation in Underground Environments}

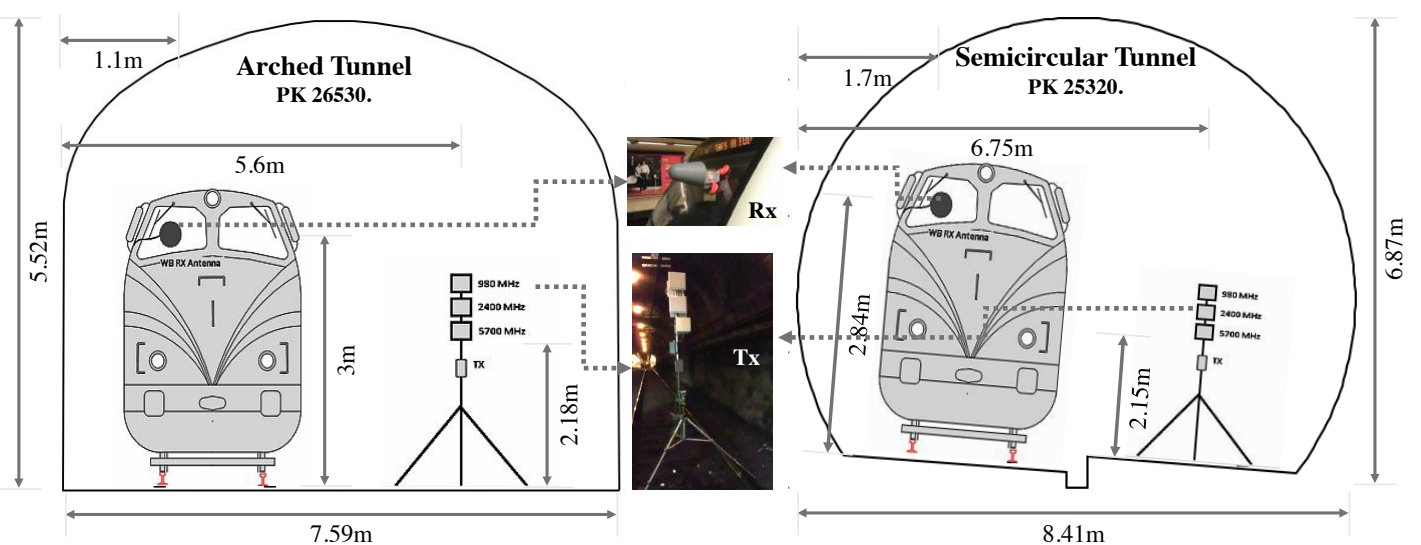

Figure 4.1. Measurement setup and photographs in the arched and semicircular tunnels.

panel antenna manufactured by L-COM. Three most commonly used frequencies are involved in the measurements, three suites of transmitting systems are assembled, which working at $920 \mathrm{MHz}, 2400 \mathrm{MHz}$, and $5705 \mathrm{MHz}$. These transmitting systems are fastened on a tripod to ensure stability during the test. On the receiver side, the wideband log-periodic antenna HL025 is adsorbed on the trains windshield and connect to an Agilent N9912A spectrum analyzer in the train's cabin to capture the power of the receiving signal versus the distance for offline processing. Moreover, the train moves forward at low speeds $(<10 \mathrm{~km} / \mathrm{h})$ and the received power is recorded. Fig. 4.1 presents the measurement setup and photographs in two tunnels. The datasheet of the transmitting system and the receiving system are listed in Table 4.1.

During the measurements, two main potential factors may affect the propagation in the tunnel are considered: frequency and polarization. Thus, $920 \mathrm{MHz}$, $2400 \mathrm{MHz}$, and $5705 \mathrm{MHz}$ are tested as the carrier frequencies to evaluate the influence of the frequency. On the other hand, vertical polarization (V-pol.) and horizontal polarization (H-pol.) are measured separately in the arched tunnel with same Radius of Curvature (RoC) (500m) to compare the impact of different polarizations. The antennas are set to be horizontal polarization during the 


\section{Propagation in Underground Environments}

measurements in the semicircular tunnel with two RoC (300 m and $500 \mathrm{~m})$ at the three frequencies. The sampling intervals are shorter than one wavelength, and repeated measurements are taken to collect sufficient samples for the study of the propagation behavior. The small-scale fading is stripped from the received power by averaging samples over 40 wavelengths [127] to clarify the large-scale fading property specified in the real curved tunnels. Moreover, the lowest average signal-to-noise-ratio (SNR) is about $30 \mathrm{~dB}$. Therefore a reliable evaluation of the channel parameters is feasible.

\subsubsection{Measurement Results Analysis}

The measurement results are presented in Fig. 4.2 after the post-processing. There are some comparisons can be made based on the results to discuss the influences of the following factors:

First, the propagation loss of horizontally polarized wave should be less than that of the vertically polarized wave based on the modal analysis in a straight tunnel, where the width of the tunnel is larger than its height. However, by comparing the subfigures in the left column of Fig. 4.2, the horizontal polarization in the curved tunnel seems to result in relatively larger propagation loss than the vertical polarization. This opposite result implies that the horizontal polarization is more sensitive to the curvature than the vertical way. Then, as shown in the right column of Fig. 4.2, the green lines with the $300 \mathrm{~m}$ RoC presents much steeper than the yellow lines with the $500 \mathrm{~m}$ RoC, which means that the wave propagated in the more curved tunnel can obtain the larger loss. Similar results are stated in $[4,123,121,118]$. Next, If we compare the dotted lines in Fig. 4.2 at the same frequencies, which are all in the horizontal polarization and in the tunnels with $500 \mathrm{~m}$ radius curvature, the waves in the curved arched tunnels experience smaller attenuations than that in the semicircular tunnels. Last but not least, as the waveguide theory indicates, wave propagation at higher frequencies experiences the less attenuation. Whereas the global losses at three frequencies are quite close in the practical tests with the same polarization, tunnel type, RoC. It can be explained that the higher frequencies are more sensitive to the curved part of the tunnel, which compensates the losses. Regarding the fading, higher frequencies in 
Arched Tunnel

Radius of curvature: $500 \mathrm{~m}$

- - - Horizontal polarization
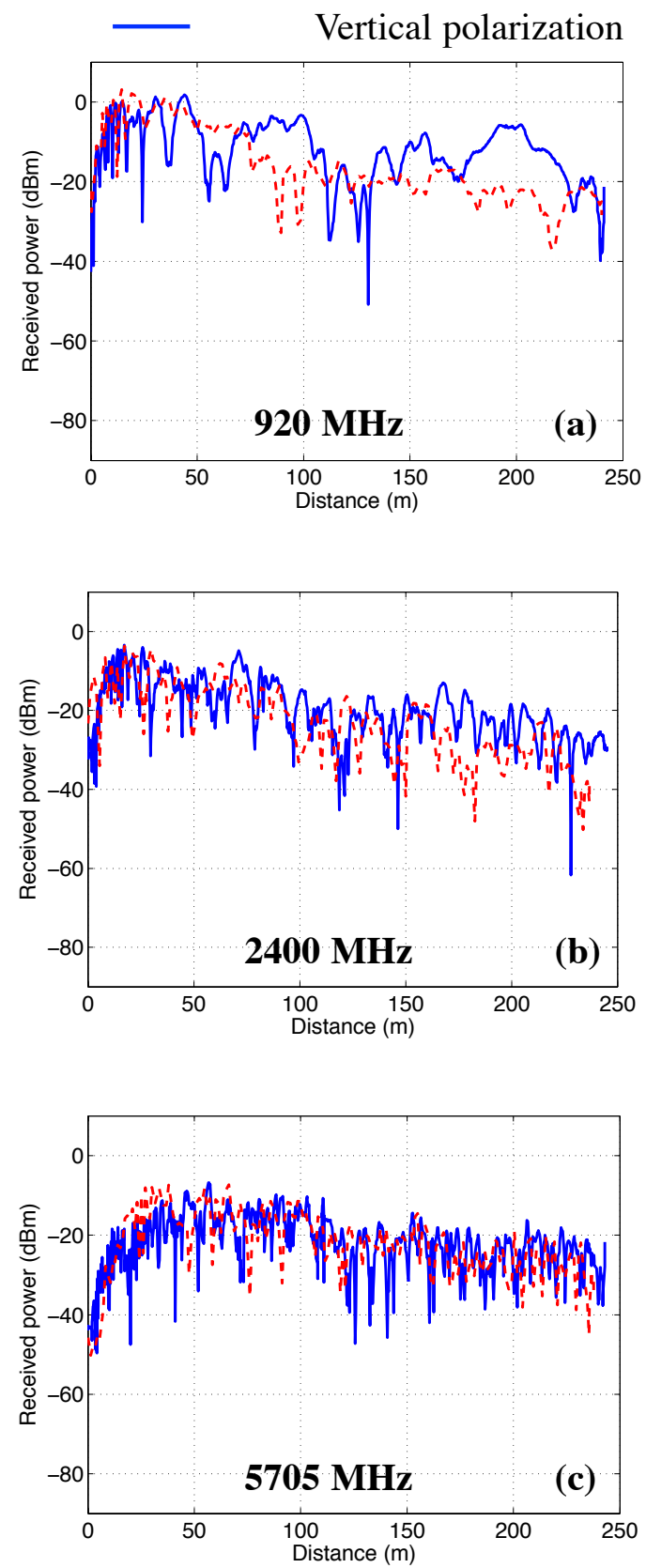

Semicircular Tunnel

Horizontal polarization

Radius of curvature: $300 \mathrm{~m}$

Radius of curvature: $500 \mathrm{~m}$
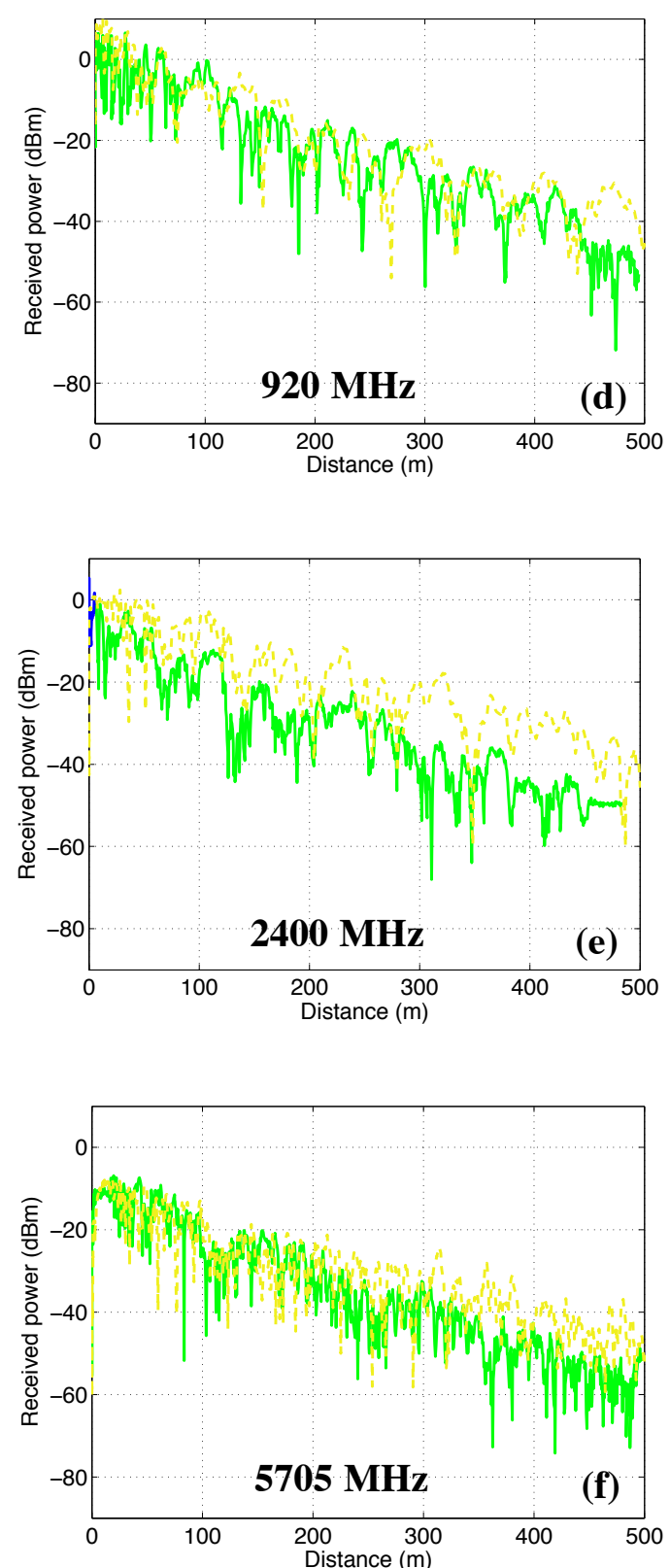

Figure 4.2. Measured received power (small-scale fading removed). Comparison with different polarizations in the traditional curved arched tunnel with $500 \mathrm{~m}$ RoC at: (a) $920 \mathrm{MHz}$, (b) $2400 \mathrm{MHz}$, and (c) $5705 \mathrm{MHz}$; Comparison with different radii of curvature in semicircular tunnels with horizontal polarization at (d) $920 \mathrm{MHz}$, (e) $2400 \mathrm{MHz}$, and (f) $5705 \mathrm{MHz}$. 


\section{Propagation in Underground Environments}

the curved tunnel have suffered fading as severe as in the straight tunnel. These analyses based on the measurement results pave the way to model the large scale fading of the propagation channel in the curved tunnels.

\section{Path Loss Exponent (PLE) and Shadow Fading Extraction}

The statistics of large-scale fading is described in terms of a mean path loss and a log-normally distributed variation about the mean in the log-distance path loss model, which provide a way to estimate the path loss as a function of distance. The PLE and the variation are depicted as mentioned earlier:

$$
P_{L}(d)=P_{\mathrm{Tx}}-P_{\mathrm{Rx}}=P_{0}+10 n \log _{10}\left(\frac{d}{d_{0}}\right)+X_{\sigma}
$$

where $d$ is the separation distance between Tx and Rx along the track of the tunnel. $P_{L}(d)$ is the difference between the transmitting power and receiving power, which is so-called "path loss". $P_{0}$ is the referenced power at the reference distance $d_{0} . n$ represents the PLE. $X_{\sigma}$ is the log-normally distributed variation on the path loss mean. After the averaging over 40 wavelengths, the small-scale fading is removed. Then the PLE $n$ can be obtained through the least-square regression. An example of the least-square fitting of $n$ in the arched tunnel at $5705 \mathrm{MHz}$ with horizontal polarization is illustrated in Fig. 4.3. Then the variations on the mean path loss can be evaluated. In our case, the transmitting system and receiving system always have a direct Line-of-Sight (LOS) path. Thus the variations can be considered to the shadow fading with a standard deviation $\sigma$. The probability density (PDF) of the shadow fading in the arched tunnel at $5705 \mathrm{MHz}$ with horizontal polarization is illustrated in Fig. 4.3, which follows a log-normal distribution.

The standard deviations $(\sigma)$ of the shadow fading are extracted based on the averaging of repeated measurements and listed Table 4.2 and Table 4.3.

\section{Autocorrelation and Cross-correlation}

Besides the distribution, the autocorrelation of shadow fading is another key 


\section{Propagation in Underground Environments}
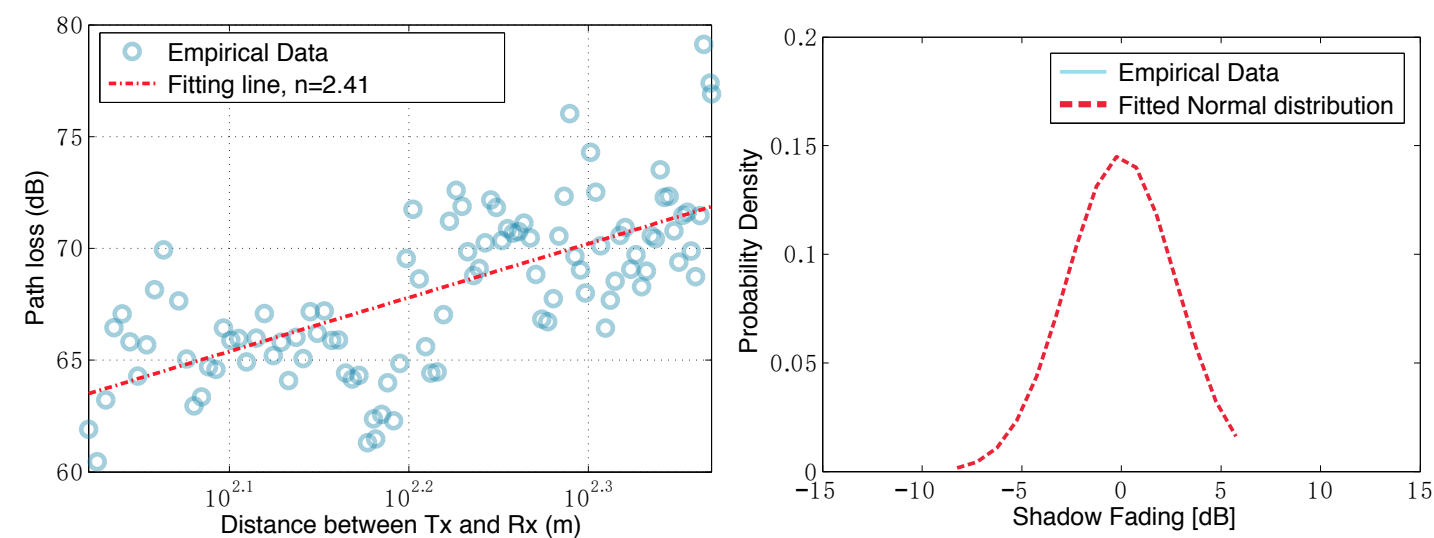

Figure 4.3. Path loss and probability density of shadow fading in the arched tunnel at $5705 \mathrm{MHz}$ with horizontal polarization.

point, which is of interest for power control and base station planning. When the transmitter is fixed, the autocorrelation is defined as the correlation of the shadow fading of the received signal at different positions. The autocorrelation is used to described by the correlation coefficient as follows:

$$
\rho_{1,2}=\frac{\mathrm{E}\left\{S\left(d_{1}\right) S\left(d_{2}\right)\right\}}{\sigma\left(d_{1}\right) \sigma\left(d_{2}\right)}
$$

where $S\left(d_{1}\right)$ and $\sigma\left(d_{1}\right)$ are the value of shadow fading and standard deviation at the distance of $d_{1}$, respectively; $S\left(d_{2}\right)$ and $\sigma\left(d_{2}\right)$ are the value of shadow fading and standard deviation at the distance of $d_{2}$, respectively.

The autocorrelation coefficient is commonly defined by the exponential model and 802.16J model. The exponential model is proposed in [128], and admitted in the standard [129, 130]. The standard IEEE 802.16J is better known as the Worldwide Interoperability for Microwave Access (WiMAX). The exponential model is defined as follow:

$$
\rho_{\exp }(\Delta d)=\exp \left(-|\Delta d| / d_{c o r}\right)
$$

The 802.16J model is only have a $\ln 2$ weight difference with the exponential 


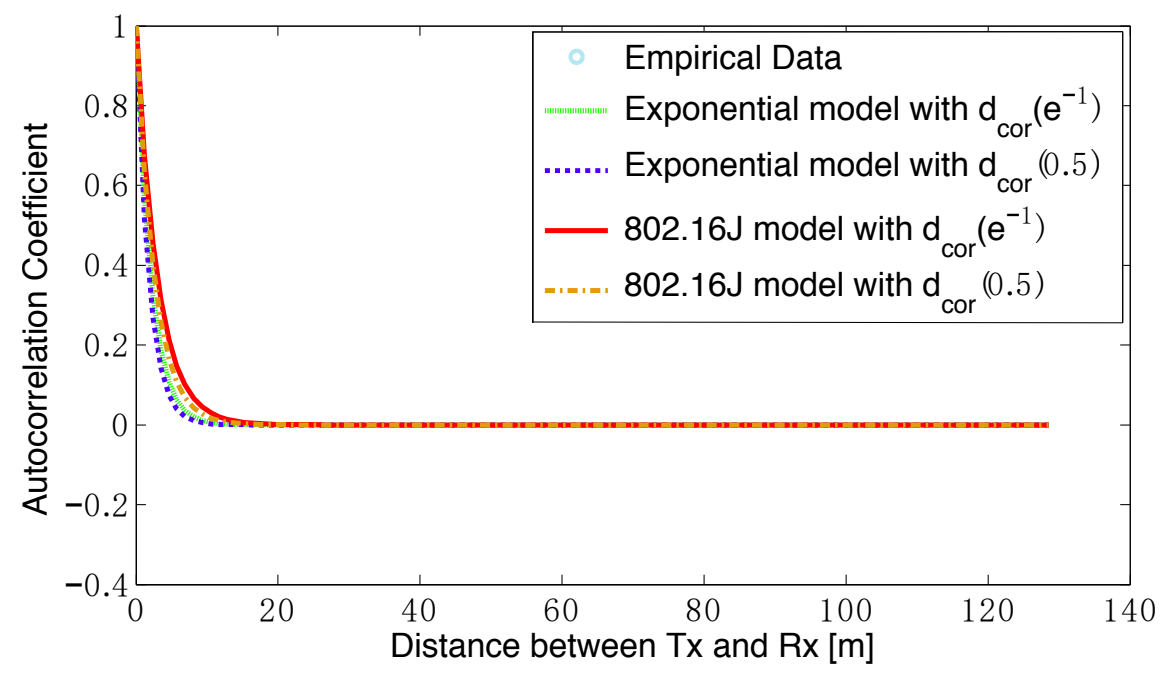

Figure 4.4. Empirical autocorrelation coefficient of shadow fading fitting in the theoretical models by LS regression in the arched tunnel at $2400 \mathrm{MHz}$ with horizontal polarization; $d_{c o r}(0.5)$ and $d_{c o r}\left(e^{-1}\right)$ represent the $d_{c o r}$ equal to 0.5 and $e^{-1}$, respectively.

model and expressed as follow:

$$
\rho_{802.16 J}(\Delta d)=\exp \left(\left(-|\Delta d| / d_{c o r}\right) \times \ln 2\right)
$$

where $\Delta d$ is the distance between the two observed positions. $d_{c o r}$ is the decorrelation distance. which is defined as the distance that the correlation is equal to $0.5\left(d_{\text {cor }}(0.5)\right)$ in $[131,132,133]$ and set to $e^{-1}\left(d_{\text {cor }}\left(e^{-1}\right)\right)$ in [128]. For the general purpose, these two definitions of decorrelation distance are involved in the both aforementioned models of autocorrelation coefficient. Then the autocorrelations are obtained by the LS fitting of the repeated measurement results. Fig. 4.4 shows an example of the empirical autocorrelation coefficients fitting in the theoretical values by using LS regression in the arched tunnel at $2400 \mathrm{MHz}$ with horizontal polarization.

The cross-correlation of shadow fading is of interested in handover techniques, macro-diversity schemes and interference power control[134]. Thus the 


\section{Propagation in Underground Environments}

cross-correlation is studied based on our measurement results as well. The crosscorrelation coefficients of shadow fading among different types of tunnels, different polarization ways and various frequencies are evaluated based on the measurement results.

The cross-correlation coefficient $\rho$ is defined by the shadow fading components of two groups of received signals as follow:

$$
\rho=\frac{\sum_{i=1}^{n}\left(X_{i}-\bar{X}\right)\left(Y_{i}-\bar{Y}\right)}{\sqrt{\sum_{i=1}^{n}\left(X_{i}-\bar{X}\right)^{2}} \sqrt{\sum_{i=1}^{n}\left(Y_{i}-\bar{Y}\right)^{2}}}
$$

where $X_{i}$ and $Y_{i}$ denote the a pair of shadow fading component that are jointly wide-sense stationary. $n$ is the total number of the shadow fading component samples of the two separate received signal. In this study, $n$ is from 200 to 400 in different measurement routes. $\bar{X}$ and $\bar{Y}$ are the mean of shadow fading components. Generally, if the absolute value of the estimated cross-correlation coefficient lies in $[0,0.1]$, two random variables can be treated as uncorrelated.

\subsubsection{Large-Scale Fading Characteristics}

Based on the above parameter extraction and initial evaluations base on the measurement results, the large-scale fading characteristics of wave propagation in curved tunnels can be quantitatively analyzed and discussed in this section.

\section{PLE and shadow fading distribution in curved arched tunnels}

Table 4.2. PLE and the standard deviations of shadow fading in the curved arched tunnel

\begin{tabular}{c|cc|cc|cc}
\hline \multicolumn{7}{c}{ Tunnel: arched; RoC: $500 \mathrm{~m}$} \\
\hline Frequency [MHz] & \multicolumn{2}{c|}{920} & \multicolumn{2}{c}{2400} & \multicolumn{2}{c}{5705} \\
\hline Polarization & Vertical & Horizontal & Vertical & Horizontal & Vertical & Horizontal \\
\hline PLE & 0.94 & 2.44 & 1.81 & 3.73 & 2.20 & 2.41 \\
\hline Std. Dev.[dB] & 4.95 & 2.18 & 3.61 & 4.12 & 3.36 & 2.74 \\
\hline \hline
\end{tabular}




\section{Propagation in Underground Environments}

Table 4.3. PLE and the standard deviations of shadow fading in the curved semicircular tunnel

\begin{tabular}{cccc|ccc}
\hline \hline \multicolumn{6}{c}{ Tunnel: semicircular; Polarization: horizontal } \\
\hline RoC $[\mathrm{m}]$ & \multicolumn{3}{c}{500} & & \multicolumn{3}{c}{300} \\
\hline Frequency $[\mathrm{MHz}]$ & 920 & 2400 & 5705 & 920 & 2400 & 5705 \\
\hline PLE & 3.36 & 5.37 & 4.94 & 4.61 & 5.50 & 4.78 \\
\hline Std. Dev.[dB] & 6.52 & 4.67 & 4.46 & 4.64 & 4.09 & 5.18 \\
\hline \hline
\end{tabular}

Firstly, the PLEs $n$ and the standard deviations (Std. Dev.) of shadow fading distributions $\sigma$ in the two types of tunnels with different frequencies, radiuses of curvature, and polarizations are calculated and listed in Table 4.2 and Table 4.3. Through the comparisons of these two critical parameters of large-scale fading, the following discussions can be addressed:

i. In most cases of wave propagation, the attenuation along the distance is greater than the case of free-space propagation, which means the PLE is larger than 2 ( $n=2$ for free-space propagation), except a waveguide effect is established in a specific environment, such as a straight tunnel or corridor. These kinds of confined scenarios lead to a $n$ small than 2 . Whereas most of the path loss components we obtain in the curved tunnel are larger than 2 , although curved tunnels are also confined, which reveals that the waveguide effect is reduced by the curve part of the tunnel, which results in an excess loss of propagation compared to the straight case.

ii. The PLEs in the curved arched tunnel are from 0.94 to 3.73, which are generally smaller than the values of PLEs from 3.36 to 5.50 in the case of curved semicircular tunnel. Even though the constructions of modern subway systems preferentially adopt the TBM semicircular tunnel than the old-fashioned arched tunnel, the PLEs are larger. Thus wave propagations may experience more propagation loss in semicircular tunnel.

iii. The PLEs at higher frequencies are slightly larger than those at lower frequencies in both types of tunnels. Since the higher frequencies experience smaller path loss in the straight tunnels due to the waveguide effect, it is worth noting that the tunnel curve may suppress the waveguide effect. An 


\section{Propagation in Underground Environments}

extreme example is reported in [135] that the path loss in proportion to the frequency in curved tunnels. Therefore, the higher frequencies are more sensitive to the curvature of the tunnel.

iv. As Table 4.3 presents, in the semicircular tunnel with the same polarization, the PLEs are 3.36 and 5.37 with $500 \mathrm{~m} \mathrm{RoC}$ at $920 \mathrm{MHz}$ and $2400 \mathrm{MHz}$, which are smaller than the values of 4.61 and 5.50 in the tunnel with $300 \mathrm{~m}$ RoC, respectively. This reveals the influence of the curvature of the tunnel on the wave propagations inside the tunnel. The authors in [115] state the larger path loss in the more curved tunnel. We can confirm it at $920 \mathrm{MHz}$ and $2400 \mathrm{MHz}$ based on our measurement results, whereas at a higher frequency, this conclusion is not entirely clear since the PLEs at $5705 \mathrm{MHz}$ are quite close.

v. In Table4.2, in the arched tunnel with the same curvature, the PLEs are from 2.41 to 3.73 with horizontal polarization, which is significantly larger than 0.94-2.20 in the case of the vertical polarization. This is consistent as mentioned above that the excess loss for the horizontally polarized wave is larger than the vertically polarized wave.

vi. The standard deviations of the log-normal distributed shadow fading in the curved tunnels are between 2.18 to 6.52 , which are larger than the values in most of other railway traffic scenarios. Besides, when the wave propagate in the same radiuses of curvature $(500 \mathrm{~m})$ and with the same horizontal polarizations, the standard deviations are from $2.18 \mathrm{~dB}$ to $4.12 \mathrm{~dB}$ in the curved arched tunnel, which is much smaller than the values from $4.09 \mathrm{~dB}$ to 6.52 $\mathrm{dB}$ in the curved semicircular tunnel.

\section{Integrations and Comparisons}

The complete integrations and comparisons of PLEs and the standard deviations of the variations on the path loss mean among various tunnels and other rail traffic scenarios are summarized in Table 4.4 between $900 \mathrm{MHz}$ and $5700 \mathrm{MHz}$ bands. 


\section{Propagation in Underground Environments}

Firstly, it can be found that the smaller PLEs are always obtained in the straight tunnels than in the curved tunnel regardless of the tunnel's cross section. Furthermore, among the straight tunnels, the subway tunnel gets the worst propagation condition with the largest PLE and standard deviation of the variations; then railway tunnel is relatively better than the subway tunnel, and the smallest PLE and variations are obtained in the road tunnel. This is determined by the dimensions of the space of the tunnel, where the road tunnel generally has the largest cross section and smallest dimensions in the subway. The PLEs and standard deviations in the curved railway tunnel are also greater than the curved subway tunnel. Then, the large-scale fading parameters in the most widely used rail traffic scenarios, such as train station, crossing bridge, cutting, and viaduct are also listed and compared with tunnels in Table 4.4. The curvature of tunnel results in the excess loss, since the PLE in the curved tunnel is relatively larger than in the other rail traffic scenarios, especially the curved arched tunnel. The standard deviation of the variations in the curved arched tunnel is relatively closer to the crossing bridge and cutting scenarios, but larger than the case of the train station and viaduct scenarios. This indicates that just like the steep walls on both sides of the track in the crossing bridge and cutting scenarios, the route and walls of the curved tunnel can also lead to the extensive reflections, diffractions, and scatterings to amplify the variations on the path loss mean.

Table 4.4 is a comprehensive evaluation of the large-scale fading characteristics in the most common rail traffic propagation scenarios, which could helpful to develop and deploy the new wireless communication systems inside various tunnels. For example, more relay systems should be deployed for the wireless network planning inside the subway tunnels; more base stations (BS) or access points (APs) should be arranged in the railway tunnel than the road tunnel.

In addition, the comparisons among the propagation in the curved tunnel scenario with in the other ten standard scenarios in the WINNER model [130] are involved as shown in Table 4.5. The PLEs and standard deviations of shadow fading are listed and compared again. It is clear that the PLEs in the curved tunnel are larger than in the standard scenarios in WINNER in most cases. On the other hand, the standard deviations of the variations in the curved tunnel scenario are close to the most LOS cases in WINNER model, but not as large 


\section{Propagation in Underground Environments}

as in the NLOS case or Outdoor to Indoor scenarios. Thus, the existing largescale fading models cannot exactly fit the characteristics of the propagation in the real curved tunnels. Therefore the propagation characterization based on our measurements is worth be considered for the system design and network planning of wireless communication systems in the curved subway tunnels.

Table 4.4. PLEs and standard deviations of shadow fading distribution among tunnels and various propagation traffic scenarios

\begin{tabular}{|c|c|c|c|c|}
\hline & Traffic scenario & Freq. $[\mathrm{MHz}]$ & PLE & Std. Dev. $[\mathrm{dB}]$ \\
\hline \multirow{9}{*}{ 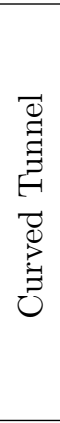 } & \multirow{3}{*}{ Subway Arched } & 920 & $0.94-2.44$ & $2.18-4.95$ \\
\hline & & 2400 & $1.81-3.73$ & $3.61-4.12$ \\
\hline & & 5705 & $2.20-2.41$ & $2.74-3.36$ \\
\hline & \multirow{3}{*}{ Subway Semicircular } & 920 & $3.36-4.61$ & $4.64-6.52$ \\
\hline & & 2400 & $5.37-5.50$ & $4.09-4.67$ \\
\hline & & 5705 & $4.78-4.94$ & $4.46-5.18$ \\
\hline & Railway Arched & $954-2000$ & $0.55-2.97$ & $4.87-5.00$ \\
\hline & \multirow{2}{*}{ Subway Arched } & 2400 & Wide: 5.49 & 2.75 \\
\hline & & 5000 & Narrow: 7.13 & 4.17 \\
\hline \multirow{4}{*}{ 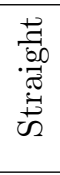 } & Road Tunnel & $2800-5000$ & $0.57-0.82$ & 2.70 \\
\hline & Railway Tunnel & $954-2000$ & $1.40-2.03$ & $4.36-4.95$ \\
\hline & \multirow[t]{2}{*}{ Subway Tunnel } & \multirow[t]{2}{*}{$945-2650$} & LOS: $1.65-1.94$ & $3.40-4.60$ \\
\hline & & & NLOS: $3.20-7.28$ & 5.47 \\
\hline & Train station & 930 & Extended Hata ${ }^{1}$ & $1.57-3.92$ \\
\hline & Crossing bridge & 930 & Extended Hata ${ }^{2}$ & $1.88-4.73$ \\
\hline & \multirow{2}{*}{ Cutting } & \multirow{2}{*}{930} & Deep: $13.05 e^{-0.039\left(w_{u p}-w_{\text {down }}\right)}$ & $2.10-$ \\
\hline & & & Low: $1.66 w_{\text {down }}{ }^{2}-58.51 w_{\text {down }}+517.6$ & 4.50 \\
\hline & Viaduct & 930 & $\begin{array}{l}2.36-0.43 H, d \leq 400 \mathrm{~m} \\
16.5-0.2 H, d>400 \mathrm{~m}\end{array}$ & $\begin{array}{l}2.00- \\
2.32\end{array}$ \\
\hline \multicolumn{5}{|c|}{$\begin{array}{l}\text { Remarks: Excess loss }[\mathrm{dB}]:{ }^{1}: 0.37-21.80 ;{ }^{2} 5.96-14.18 ; w_{u p}: \text { upper width; } w_{\text {down }}: \text { lower width; } H \text { : viaduct height. } \\
\text { References: }[92,136,137,138,139,140,141,142,143,144,145,146,147,148]\end{array}$} \\
\hline
\end{tabular}

\section{Autocorrelation and Cross-correlation Characteristics}

The statistics of the decorrelation distances defined by $d_{c o r}(0.5)$ and $d_{c o r}\left(e^{-1}\right)$ are summarized in Table 4.6, in the different curved tunnels with different radiuses of curvature, at three frequencies, and with two polarizations. The decorrelation distances in 10th, 50th, 90th percentiles are provided in Table 4.6. In the curved semicircular tunnel, the mean values of decorrelation distances in the tunnel of 


\section{Propagation in Underground Environments}

Table 4.5. Comparison of large-scale fading characteristics between curved arched tunnel scenario and standard scenarios in WINNER

\begin{tabular}{|c|c|c|c|c|}
\hline \multicolumn{2}{|c|}{ Propagation scenario } & Freq. [GHz] & PLE & Std. Dev. [dB] \\
\hline \multirow{3}{*}{ Curved Subway Tunnel } & \multirow{3}{*}{ Arched } & 0.92 & $0.94-2.44$ & $2.18-4.95$ \\
\hline & & 2.4 & $1.81-3.73$ & $3.61-4.12$ \\
\hline & & 5.705 & $2.20-2.41$ & $2.74-3.36$ \\
\hline \multirow{3}{*}{ Curved Subway Tunnel } & \multirow{3}{*}{ Semicircular } & 0.92 & $3.36-4.61$ & $4.64-6.52$ \\
\hline & & 2.4 & $5.37-5.50$ & $4.09-4.67$ \\
\hline & & 5.705 & $4.78-4.94$ & $4.46-5.18$ \\
\hline \multirow{2}{*}{$\begin{array}{c}\text { Indoor Office/ } \\
\text { Residential }[\mathrm{A} 1]\end{array}$} & LOS & $2-6$ & 1.87 & 3.00 \\
\hline & NLOS & $2-6$ & $3.68 / 2.00$ & 4.00 \\
\hline $\begin{array}{c}\text { Indoor to Outdoor/ } \\
\text { Outdoor to Indoor } \\
{[\mathrm{A} 2 / \mathrm{B} 4 / \mathrm{C} 4]}\end{array}$ & NLOS & $2-6$ & 2.27 & 7.00 \\
\hline Typical Urban & LOS & $2-6$ & $2.27 / 4.00$ & 3.00 \\
\hline Micro-cell [B1] & NLOS & $2-6$ & $\max \left(2.8-0.0024 d_{k}, 1.84\right)$ & 4.00 \\
\hline Large Indoor & LOS & $2-6$ & 1.39 & 3.00 \\
\hline Hall [B3] & NLOS & $2-6$ & 3.78 & 4.00 \\
\hline \multirow{2}{*}{ Suburban $[\mathrm{C} 1]$} & LOS & $2-6$ & $2.38 / 4.00$ & $4.00 / 6.00$ \\
\hline & NLOS & $2-6$ & $\left(4.49-0.655 \lg \left(h_{B S}\right)\right)$ & 8.00 \\
\hline Typical Urban & LOS & $2-6$ & $2.60 / 4.00$ & $4.00 / 6.00$ \\
\hline Macro-Cell [C2] & NLOS & $2-6$ & $\left(4.49-0.655 \lg \left(h_{B S}\right)\right)$ & 8.00 \\
\hline Rural & LOS & $2-6$ & $2.15 / 4.00$ & $4.00 / 6.00$ \\
\hline Macro-Cell [D1] & NLOS & $2-6$ & 2.51 & 8.00 \\
\hline $\begin{array}{c}\text { Moving Networks } \\
\text { BS-MRS, Rural [D2a] }\end{array}$ & LOS & $2-6$ & $2.15 / 4.00$ & 4.00 \\
\hline Remarks: $d_{k} \leq 400 \mathrm{~m}$ & $S=25 \mathrm{n}$ & & & \\
\hline
\end{tabular}

$500 \mathrm{~m}$ RoC are $11.19-16.75 \mathrm{~m}$ at $920 \mathrm{MHz}, 1.41-1.83 \mathrm{~m}$ at $2400 \mathrm{MHz}$, and 4.13$5.78 \mathrm{~m}$ at $5705 \mathrm{MHz}$. Which are mainly larger than the relative decorrelation distances when the $\mathrm{RoC}$ is $300 \mathrm{~m}: 6.35-8.87 \mathrm{~m}$ at $920 \mathrm{MHz}, 2.05-2.56 \mathrm{~m}$ at 2400 $\mathrm{MHz}, 3.23-5.50 \mathrm{~m}$. This means that the larger radiuses of curvature, the longer decorrelation distance, and better for decorrelation of the received signal. Besides, the mean values of decorrelation distances in the curved arched tunnel are 1.86$4.10 \mathrm{~m}$ at $920 \mathrm{MHz}, 2.26-3.43 \mathrm{~m}$ at $2400 \mathrm{MHz}$, and $1.79-3.28 \mathrm{~m}$ at $5705 \mathrm{MHz}$, which are less distance than those in the urban macro scenarios, $32 \mathrm{~m}, 45 \mathrm{~m}$, and $97 \mathrm{~m}$ as reported in [149], and in other outdoor railway environments, such as viaduct in $14.81-131.6 \mathrm{~m}$ as the author in $[130,150,151]$ state. Therefore, the shadow fading component along the distance varies fairly faster in the curved subway tunnels than in the open area. 
4. Propagation in Underground Environments

Table 4.6. Decorrelation distance statistic in the curved arched and semicircular tunnel

\begin{tabular}{|c|c|c|c|c|c|c|c|c|c|c|c|c|c|}
\hline \multicolumn{14}{|c|}{ Tunnel: arched; RoC: $500 \mathrm{~m}$} \\
\hline \multirow{2}{*}{\multicolumn{2}{|c|}{$\begin{array}{c}\text { Frequency } \\
\text { Polarization }\end{array}$}} & \multicolumn{4}{|c|}{$920 \mathrm{MHz}$} & \multicolumn{4}{|c|}{$2400 \mathrm{MHz}$} & \multicolumn{4}{|c|}{$5705 \mathrm{MHz}$} \\
\hline & & \multicolumn{2}{|c|}{ Vertical } & \multicolumn{2}{|c|}{ Horizontal } & \multicolumn{2}{|c|}{ Vertical } & \multicolumn{2}{|c|}{ Horizontal } & \multicolumn{2}{|c|}{ Vertical } & \multicolumn{2}{|c|}{ Horizontal } \\
\hline \multicolumn{2}{|c|}{ Correlation } & 0.5 & $e^{-1}$ & 0.5 & $e^{-1}$ & 0.5 & $e^{-1}$ & 0.5 & $e^{-1}$ & 0.5 & $e^{-1}$ & 0.5 & $e^{-1}$ \\
\hline \multirow{4}{*}{$d_{\text {cor }}[\mathrm{m}]$} & $10 \%$ & 2.47 & 3.12 & 1.51 & 1.51 & 1.66 & 1.95 & 1.96 & 1.96 & 0.89 & 1.18 & 0 & 1.18 \\
\hline & $50 \%$ & 3.39 & 4.19 & 2.04 & 2.04 & 2.24 & 2.82 & 1.96 & 3.23 & 1.45 & 2.02 & 1.18 & 1.18 \\
\hline & $90 \%$ & 4.19 & 4.81 & 2.04 & 3.51 & 3.11 & 4.21 & 3.23 & 3.23 & 3.18 & 6.64 & 3.49 & 3.49 \\
\hline & Mean & 3.27 & 4.10 & 1.86 & 2.62 & 2.26 & 3.43 & 2.46 & 3.16 & 1.89 & 3.28 & 1.79 & 2.08 \\
\hline \multicolumn{14}{|c|}{ Tunnel: semicircular; Polarization: horizontal } \\
\hline \multicolumn{2}{|c|}{$\mathrm{RoC}$} & \multicolumn{6}{|c|}{$300 \mathrm{~m}$} & \multicolumn{6}{|c|}{$500 \mathrm{~m}$} \\
\hline \multicolumn{2}{|c|}{ Frequency } & \multicolumn{2}{|c|}{$920 \mathrm{MHz}$} & \multicolumn{2}{|c|}{$2400 \mathrm{MHz}$} & \multicolumn{2}{|c|}{$5705 \mathrm{MHz}$} & \multicolumn{2}{|c|}{$920 \mathrm{MHz}$} & \multicolumn{2}{|c|}{$2400 \mathrm{MHz}$} & \multicolumn{2}{|c|}{$5705 \mathrm{MHz}$} \\
\hline \multicolumn{2}{|c|}{ Correlation } & 0.5 & $e^{-1}$ & 0.5 & $e^{-1}$ & 0.5 & $e^{-1}$ & 0.5 & $e^{-1}$ & 0.5 & $e^{-1}$ & 0.5 & $e^{-1}$ \\
\hline \multirow{4}{*}{$d_{\text {cor }}[\mathrm{m}]$} & $10 \%$ & 3.89 & 5.30 & 1.59 & 1.86 & 2.96 & 2.96 & 9.83 & 10.50 & 0.73 & 0.73 & 2.96 & 4.00 \\
\hline & $50 \%$ & 5.74 & 6.67 & 1.86 & 2.33 & 2.96 & 3.44 & 10.50 & 11.38 & 0.73 & 2.66 & 4.00 & 4.84 \\
\hline & $90 \%$ & 8.79 & 11.50 & 2.80 & 3.30 & 3.44 & 9.18 & 11.08 & 37.77 & 2.66 & 2.66 & 4.84 & 6.60 \\
\hline & Mean & 6.35 & 8.87 & 2.05 & 2.56 & 3.23 & 5.50 & 11.19 & 16.75 & 1.41 & 1.83 & 4.13 & 5.78 \\
\hline
\end{tabular}

To evaluate the goodness of fit (GoF) between the exponential model and 802.16J model, the mean error (ME), standard deviation (Std.) and and root mean square error (RMSE) are involved within all the measurement results with two definitions of $d_{c o r}$. The statistic values are calculated and presented in Table 4.7. Since the mean values of ME are close to 0 in all cases, and the mean values of RMSE are near to 0.15 , the autocorrelations of the measurement fit both models quite well. However, the mean values of RMSE in the exponential model is always slightly larger than the values in 802.16J model, which implies that the $802.16 \mathrm{~J}$ model fits better with the measurement data and thus a more appropriate model for real curved subway tunnels. In the meantime, comparison shows the decorrelation distance defined by $d_{c o r}\left(e^{-1}\right)$ alway has a better GoF than the definition of $d_{c o r}(0.5)$, regardless of the type of tunnel.

Table 4.8 presents the statistic results of cross-correlation characterization of the shadow fading in curved tunnels with various configurations. It can be observed the cross-correlation values in the tunnel with $300 \mathrm{~m}$ RoC are smaller than in the curved tunnel with larger RoC $(500 \mathrm{~m})$, and the horizontally polarized waves also have smaller cross-correlation values than the cases of vertical polarization. However, the both differences are slight. It implies that the variation of curved radius or polarizations in the curved tunnel may only bring little influences on shadowing cross-correlation. Moreover, the cross-correlation coefficients are smaller 
Table 4.7. Statistic values of the ME, Std., and RMSE between measurements and the exponential model and the 802.16J model

\begin{tabular}{|c|c|c|c|c|c|c|c|c|c|}
\hline \multirow{3}{*}{\multicolumn{2}{|c|}{$\begin{array}{c}\text { Tunnel Type } \\
\text { Model } \\
\text { Correlation }\end{array}$}} & \multicolumn{4}{|c|}{ arched } & \multicolumn{4}{|c|}{ semicircular } \\
\hline & & \multicolumn{2}{|c|}{ Exponential } & \multicolumn{2}{|c|}{$802.16 \mathrm{~J}$} & \multicolumn{2}{|c|}{ Exponential } & \multicolumn{2}{|c|}{$802.16 \mathrm{~J}$} \\
\hline & & 0.5 & $e^{-1}$ & 0.5 & $e^{-1}$ & 0.5 & $e^{-1}$ & 0.5 & $e^{-1}$ \\
\hline \multirow{4}{*}{ ME } & $10 \%$ & 0.016 & 0.018 & 0.022 & 0.027 & 0.024 & 0.030 & .033 & 0.043 \\
\hline & $50 \%$ & 012 & 0.017 & 0.018 & 0.025 & 0.013 & 0.022 & 0.021 & 0.032 \\
\hline & $90 \%$ & 007 & 0.012 & 0.010 & 0.018 & .005 & 0.006 & 07 & 0.009 \\
\hline & Mean & 012 & 0.016 & 0.017 & 0.024 & 0.012 & 0.017 & 0.017 & 0.024 \\
\hline \multirow{4}{*}{ Std. } & $10 \%$ & 0 & 96 & 0.100 & 0.091 & 1 & 94 & 092 & 0.086 \\
\hline & $50 \%$ & 148 & 0.140 & 0.141 & 0.134 & 7 & 0.104 & 0.103 & 0.100 \\
\hline & & & & & & & & 67 & 0.256 \\
\hline & Mean & 8 & 0.016 & 0.150 & 0.144 & 0.147 & 0.140 & 0.138 & 0.131 \\
\hline \multirow{4}{*}{ RMSE } & $10 \%$ & & 0.097 & 0.100 & 0.093 & & 0.095 & 0.092 & 0.087 \\
\hline & $50 \%$ & 148 & 0.141 & 0.142 & 0.136 & 107 & 0.104 & 0.104 & 0.101 \\
\hline & & & 0.230 & 0.225 & & & 0.272 & 0.268 & 0.260 \\
\hline & Mean & 0.158 & 0.151 & 0.151 & 0.146 & 0.148 & 0.140 & 0.140 & 0.133 \\
\hline
\end{tabular}

than 0.41 in all cases. Which indicates that the signals in different frequencies or types of tunnels are uncorrelated or with a low correlation. Furthermore, since the effects of the propagation channel on the $920 \mathrm{MHz}, 2400 \mathrm{MHz}$, and $5705 \mathrm{MHz}$ are essentially uncorrelated, frequency diversity among these frequencies can be expected for diversity gains. And more specifically, various communication system standards, such as GSM/GSM-R/LTE/TETRA/LTE-R work at $900 \mathrm{MHz}$, Wi-Fi/UMTS/LTE/CBTC/LTE-R at $2400 \mathrm{MHz}$, and CBTC, etc. at $5700 \mathrm{MHz}$ could be accepted to work together and without severe interference in the same curved tunnel. However, the cross-correlation values in the channels at the same frequency in different types of curved tunnels are quite small. Thus the channels are uncorrelated. This instead implies that the cross sections of the tunnel can largely determine the shadow fading of received signal in the curved tunnel. Therefore, the network planning in different types of curved tunnels should be designed and conducted independently. 


\section{Propagation in Underground Environments}

Table 4.8. Cross-correlation of the shadow fading between the $920 \mathrm{MHz}, 2400$ $\mathrm{MHz}$, and $5705 \mathrm{MHz}$ in curved subway tunnels

\begin{tabular}{c|c|c|c}
\hline \hline Frequency Bands & 920 vs. $2400 \mathrm{MHz}$ & 920 vs. $5705 \mathrm{MHz}$ & $2400 \mathrm{vs.} 5705 \mathrm{MHz}$ \\
\hline \hline \multicolumn{4}{c}{ Tunnel: arched; RoC: $500 \mathrm{~m}$} \\
\hline Vertical Pol. & 0.41 & 0.24 & 0.08 \\
Horizontal Pol. & 0.24 & 0.13 & 0.08 \\
\hline \multicolumn{4}{c}{ Tunnel: semicircular; Polarization: horizontal } \\
\hline Radius: $300 \mathrm{~m}$ & 0.23 & 0.12 & 0.04 \\
Radius: $500 \mathrm{~m}$ & 0.27 & 0.04 & 0.07 \\
\hline
\end{tabular}

Tunnel: arched \& semicircular; RoC: 500m; Polarization: horizontal

\begin{tabular}{c|c|c|c}
\hline Frequency Bands & $920 \mathrm{MHz}$ & $2400 \mathrm{MHz}$ & $5705 \mathrm{MHz}$ \\
\hline Arched vs Semicircular & 0.05 & 0.05 & 0.04 \\
\hline \hline
\end{tabular}

\subsection{Part II: Broadband Measurements in Sta- tion and Tunnel}

As discussed above, the tunnel is a common scenario in public transportation systems. Radio propagation modeling in this scenario is important as the wireless communication technology involved is developing at a dramatic rate. Furthermore, in subway environments, stations are also in abundance and can not be ignored. This section presents a set of broadband $(20 \mathrm{MHz})$ propagation measurements conducted in the subway system of Madrid at $980 \mathrm{MHz}$ and $2450 \mathrm{MHz}$. The measurements have been divided into two groups of testing for considering different influences on the wireless propagation in subway environments.

In the first group of testing, namely TEST I characterizes the propagation inside the subway station, with and without the metro train. The key parameters, such as RMS delay and reverberation time are compared in different situations. Then, the wireless channel from the subway station to the channel is divided into various regions with different propagation mechanisms in TEST II. The RMS delay and the number of channel taps are parameterized to establish a regionbased TDL model. Also, the reverberation time, quality factor (Q) are extracted from the RAW data to model the electromagnetic reverberation in the enclosed space, and the transition distance from the station to the tunnel is modeled based 
on the $\mathrm{Q}$ values.

\subsubsection{Measurements Scenarios and Configurations}

\section{Measurement Scenarios}

The broadband measurement campaign has been conducted in a modern subway station of line 3 in Madrid and the adjoining tunnels. The main dimensional parameters of the test environment can be found in Fig. 4.5, where the station can be roughly regarded as a reverse T-shaped block with $112 \mathrm{~m}$ in length and $16 \mathrm{~m}$ in width. Two straight TBM semicircular tunnels with the lengths over $1000 \mathrm{~m}$ have been joined at the two ends of the subway station. The cross section of the semicircular tunnel has an $4.17 \mathrm{~m}$ radius, which also has been marked in Fig. 4.5 with dimensions in detail. The test vehicle is a commercial CAF S/3000 train with $89.38 \mathrm{~m}$ long.

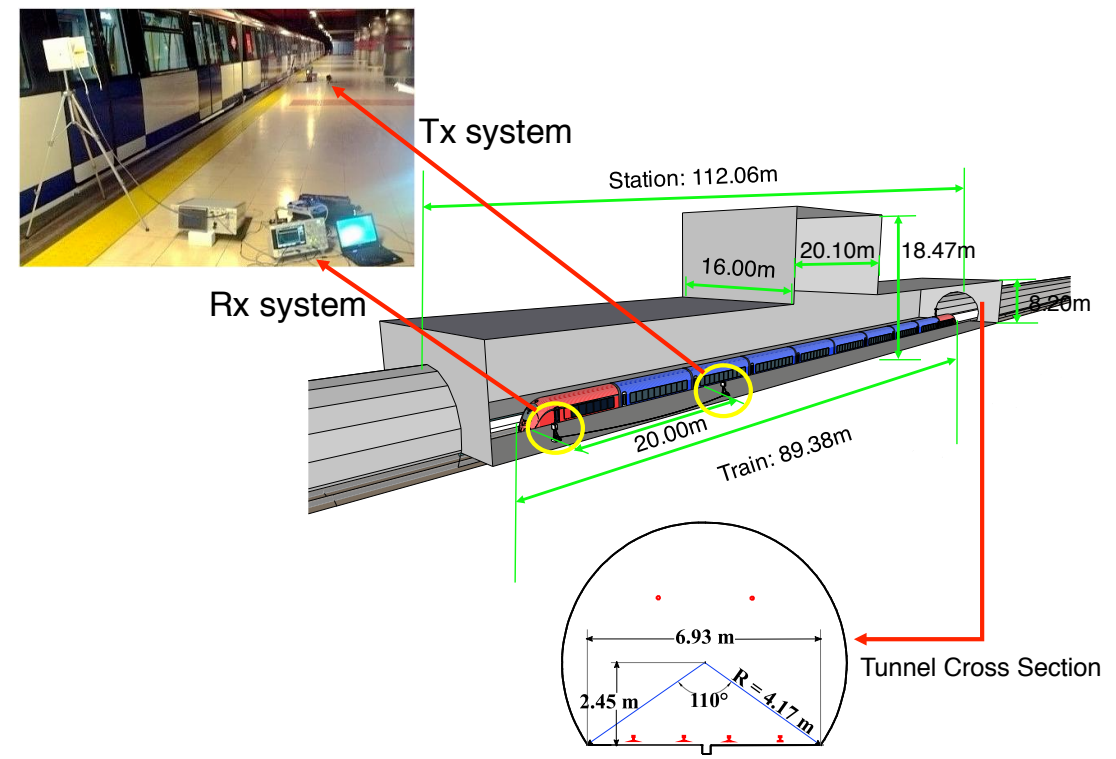

Figure 4.5. Diagrammatic sketch and photo of the measurement scenarios and setup of the 1st Test.

\section{Measurement Assignment}




\section{Propagation in Underground Environments}

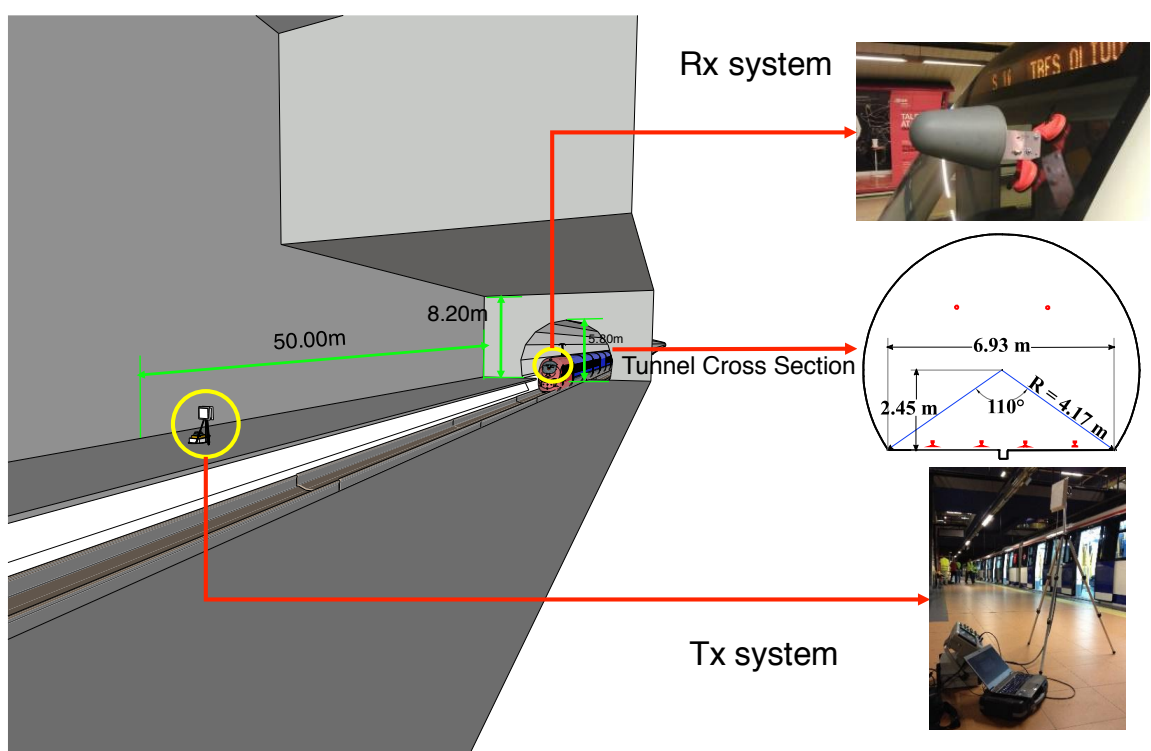

Figure 4.6. Diagrammatic sketch and photos of the measurement scenarios and setup of the 2nd Test.

Since the subway station and tunnel have different architectural structures with different interior surfaces, broadband measurements are divided into two group of testings:

- The first group testing is designed to model propagation in the station and the influence of the train body at different positions. On this test, both transmitter and receiver are placed on the platform of the station and separated $20 \mathrm{~m}$ as Fig. 4.5 shows. The train is driven on the track near to the testbed and stopped at different positions inside the station and tunnel as illustrated in Fig.4.7.

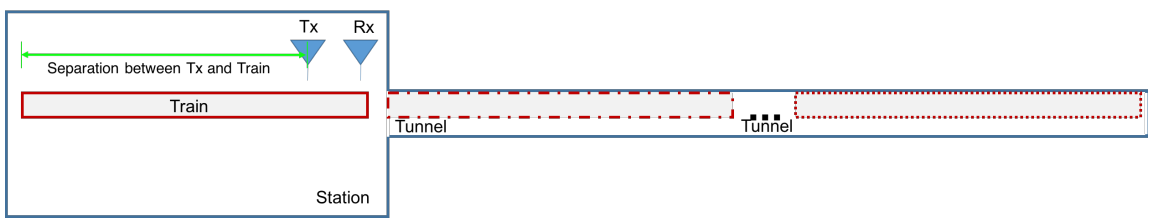

Figure 4.7. Simplified diagram of the first group testing.

- The second group testing is set to collect the channel impulse responses when the train is moving from the station into inside the tunnel. As shown 


\section{Propagation in Underground Environments}

in Fig. 4.6 and Fig.4.8, the transmitter is placed on the platform in the middle of the station. The receiver is placed in the operating cab, with the receiving antenna adsorbed on the windshield of the front car.

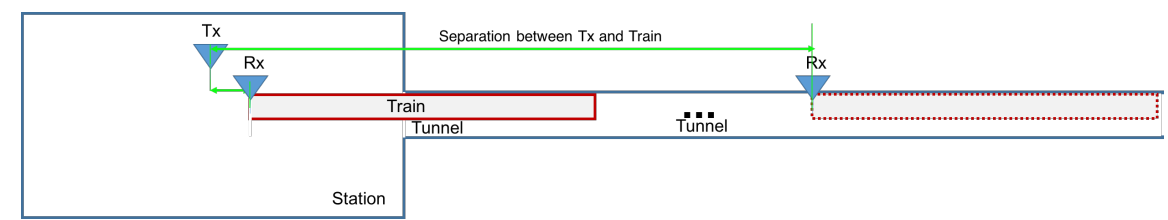

Figure 4.8. Simplified diagram of the second group testing.

In addition, both groups of testing are repeated at two frequencies: $980 \mathrm{MHz}$ and $2450 \mathrm{MHz}$, and making an average of $256 \mathrm{PDPs}$ in all positions. The results provide an accurate distribution of signal power received over a multipath channel as a function of propagation delays in the time domain.

\section{Measurement Testbeds}

The measurements have been conducted using the wideband channel sounder described in Section. 3.2.2, which is a high-performance and reconfigurable channel sounder testbed specially developed for railway measurements [78]. This channel sounder system has separate transmitter and receiver. The transmitting system is composed of the signal generator, the narrow pulse modulator, and a high power amplifier. The receiving system is equipped with a high-quality logarithmic detector, a high-performance oscilloscope, and a portable laptop computer. Detailed configuration and related parameters are listed in TABLE 4.9.

Three types of antennas have been involved in the broadband measurement campaign: a pair of L-Com HG 908P and HG2414P Flat Panel antennas are used for the fist group testing in the station; the R\&S HL025 Log-periodic antenna covers from 0.3 t0 $6 \mathrm{GHz}$ as the receiving antenna in the second group testing, and HG 908P and HG2414P are also adopted as transmitting antenna for measurements at difference frequencies. The photos and characterized radiation patterns can be found in Appendix: Fig.1 and Fig.2. 
4. Propagation in Underground Environments

Table 4.9. Channel sounder configurations.

\begin{tabular}{l|c}
\hline \hline Item & Description \\
\hline Frequency & $980 / 2450 \mathrm{MHz}$ \\
\hline IF bandwidth & $100 \mathrm{MHz}$ \\
\hline Modulation & Pulse \\
\hline Pulse width / period & $47 / 1500 \mathrm{~ns}$ \\
\hline Tx power & $980 \mathrm{MHz}: 40 \mathrm{dBm} / 2450 \mathrm{MHz}: 38 \mathrm{dBm}$ \\
\hline Noise figure & $4 \mathrm{~dB}$ \\
\hline Sensitivity & $-90 \mathrm{dBm}$ \\
\hline Demodulation & Logarithmic detector \\
\hline Synchronization & Single pulse \\
\hline \hline
\end{tabular}

During the tests, the transmitting system generates and transmits the periodic narrow pulses into the specific scenarios. Then the distorted and delayed signals from multipath are captured by the receiving system. The channel impulse response is represented by the envelope detector, and the PDPs are recorded and stored by the digital oscilloscope for the off-line processing.

\subsubsection{Measurement Results Analysis}

The RAW data stored by the digital oscilloscope is post-processed by Matlab. The measurement results are analyzed by the PDPs in different scenarios.

\section{TEST I: Influences from the Station and the Train}

The main objective of TEST I is evaluating the influence of the station and the impact of train's arriving. For these purposes, the transmitter and receiver are fixed inside the station, and the testing train is stopped at different positions along the track. The PDPs are stored for different positions of the train moving from the station into the tunnel (see Fig. 4.7). Fig. 4.9 and Fig. 4.10 show the PDPs in different cases of train's location at $2450 \mathrm{MHz}$ and $980 \mathrm{MHz}$, respectively. Firstly, propagation at higher frequency has shorter excess delay ( $80 \sim 180 \mathrm{~ns})$ than at lower frequency $(480 \sim 520 \mathrm{~ns})$. Besides, it can be found most of the PDPs at the same frequency are very similar when the train is stopped at different 


\section{Propagation in Underground Environments}

positions. Yet, the obtained PDPs at each frequency can generally be classified on the basis of three cases:

- Train in the station: the whole train body is stopped in the middle of the station.

- Train near the entrance: the train's head is located near the entrance of the station, which is closed to the transmitter and receiver.

- Train in the tunnel: the train is driven inside tunnel and very limited affect the propagation inside the subway station.

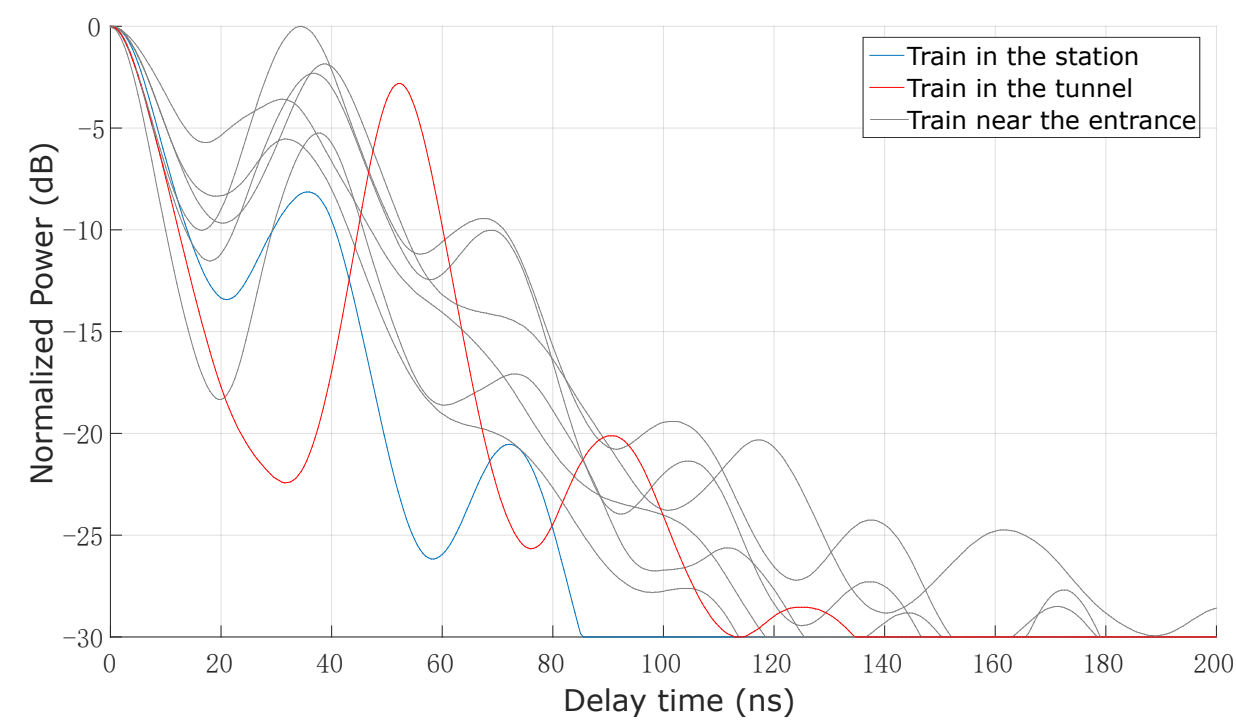

Figure 4.9. Empirical PDP samples in the subway station at $2450 \mathrm{MHz}$, when the train stopped at different positions.

Propagation in these three cases are slightly different as the highlighted PDPs present in Fig. 4.9 and Fig. 4.10. When the train is stopped in the station, the PDPs have the shortest delay spread at both frequencies, compared to the other cases. The possible reason is the train body compressing the propagation space in the station, and contributes many MPCs with short delays. When the train is approaching the entrance of the station, the absence of the train makes the propagation space greatens in this case. Hence, the PDPs present 


\section{Propagation in Underground Environments}

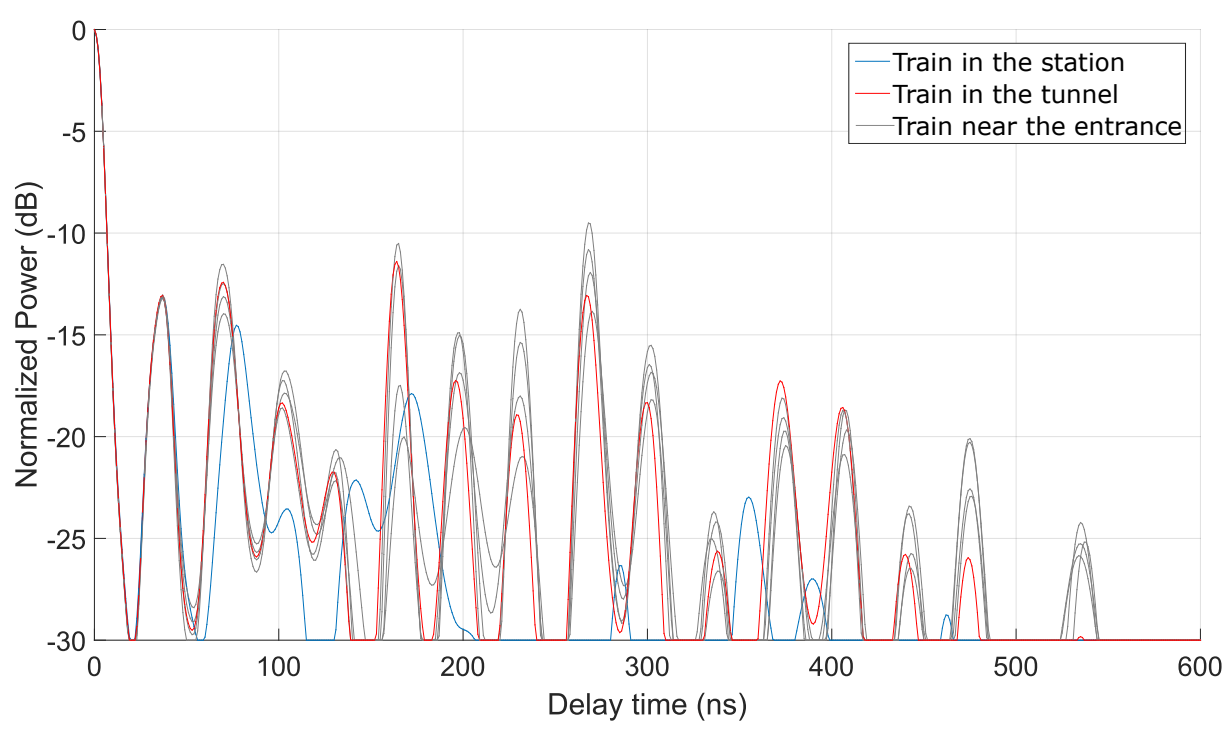

Figure 4.10. Empirical PDP samples in the subway station at $980 \mathrm{MHz}$, when the train stopped at different positions.

longer delays. Also, the reflections on train body create MPCs near the entrance, whereas this effect is highly filtered when the train is located deeply in the tunnel. Consequently, the PDPs are shorten again at $980 \mathrm{MHz}$ and $2450 \mathrm{MHz}$. Note that the power amplitude of PDP varies small in all cases.

\section{TEST II: Influence from the Tunnel}

The influence from the tunnel occurs when the receiving system is on-board the train from the subway station into the tunnel. The change of spatial structure in the subway system brings particular multipath propagation, thus this special time dispersive appearance is worthy of study. The second group testing TEST II is designed to observe the effects on the propagation from the different regions in the tunnel. Fig. 4.11 and Fig. 4.12 show the PDPs obtained at several positions along the moving path from the subway station into the tunnel at $980 \mathrm{MHz}$ and $2450 \mathrm{MHz}$, respectively. PDPs have been normalized and limited with a $-30 \mathrm{~dB}$ minimum threshold to clarify the effect from the tunnel. These figures are very relevant and clearly demonstrate how the power of multipath components varies along the train passing from the station into the deep tunnel. 


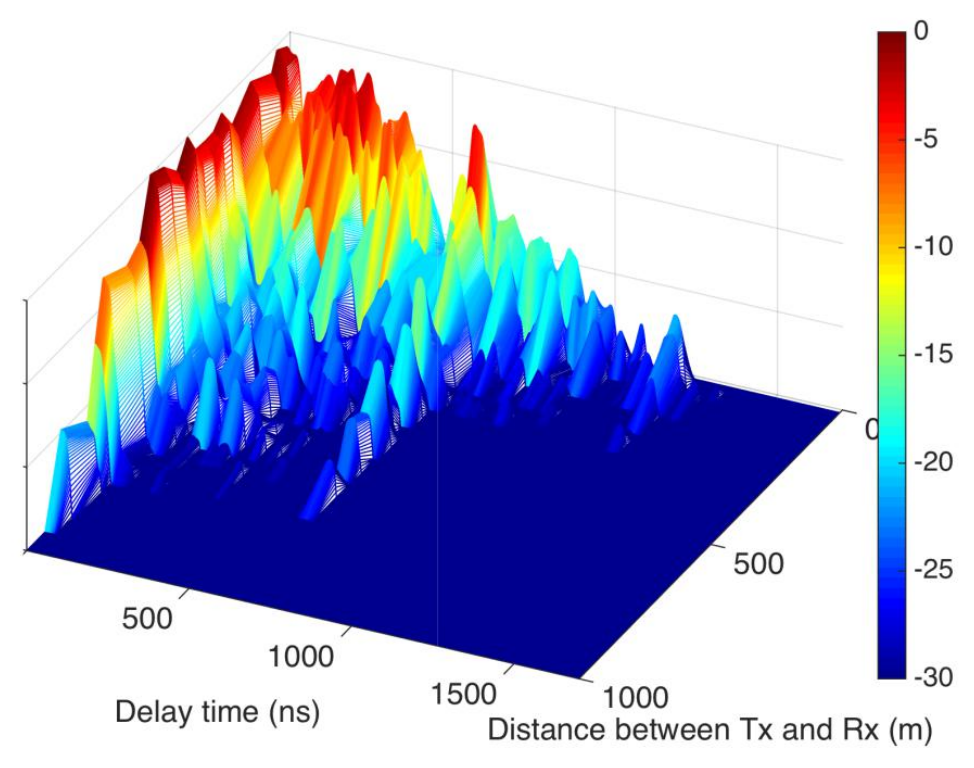

Figure 4.11. PDPs variation versus distance at $980 \mathrm{MHz}$.

It is evident that the filtering effect of the tunnel that attenuates the MPCs in the subway. This effect is clearly greater at $2450 \mathrm{MHz}$ than at $980 \mathrm{MHz}$. In the time domain, the maximum excess delay beyond $1000 \mathrm{~ns}$ at $980 \mathrm{MHz}$, while the time delay at $2450 \mathrm{MHz}$ is less than $200 \mathrm{~ns}$. Generally, the time delay spread is much longer at $980 \mathrm{MHz}$ than the delay spread in the corresponding test point at $2450 \mathrm{MHz}$, Spatially, there is no more noticeable MPCs received when the transmitting system is over $200 \mathrm{~m}$ away from the transmitter at 2450 $\mathrm{MHz}$. Whereas in the case of $980 \mathrm{MHz}$, PDPs at the positions over $500 \mathrm{~m}$ still show the visible delays. This is thus evident, the transition distance from the area with dense MPCs to the area with handful MPCs is expected to be much longer at $980 \mathrm{MHz}$ than at $2450 \mathrm{MHz}$.

\subsubsection{Characterization and Modeling}

\section{RMS Delay and Number of Channel Taps}

For digging deeper information of the propagation in subway system, the RMS delay of each PDP is calculated as follow [152]: 


\section{Propagation in Underground Environments}

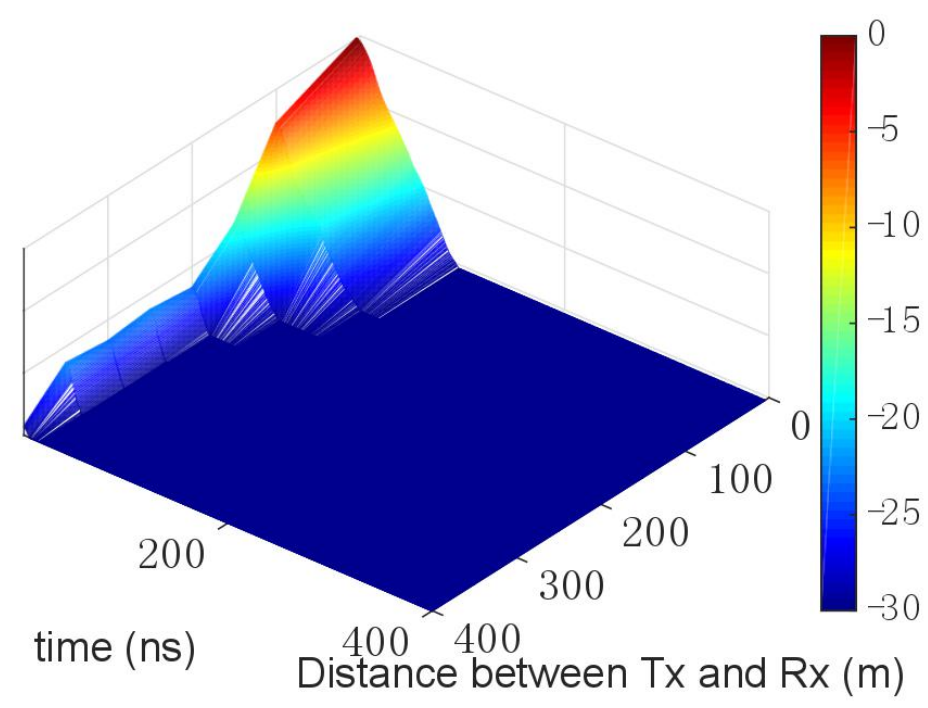

Figure 4.12. PDPs variation versus distance at $2450 \mathrm{MHz}$.

$$
\sigma_{\tau}=\sqrt{\frac{\sum_{k} P\left(\tau_{k}\right) \tau_{k}^{2}}{\sum_{k} P\left(\tau_{k}\right)}-\left(\frac{\sum_{k} P\left(\tau_{k}\right) \tau_{k}}{\sum_{k} P\left(\tau_{k}\right)}\right)^{2}}
$$

Eq. (4.6) expounds the calculation of RMS delay spread $\sigma_{\tau}$, which is the square root of difference between the second moment $(\bar{\tau})^{2}$ and first moment $\overline{\tau^{2}}$. Note $k$ represents the $k-t h$ resolvable path with the corresponding delay $\tau_{k}$ and power $P\left(\tau_{k}\right)$.

Moreover, to establish a TDL model, the number of channel taps has to be calculated on the basis of the RMS delay on each test point. A channel tap indicates the minimum delay spread in the channel that the receiver can be effectively resolved. As Section 2.2.4 discussed, the number of channel taps $L$ is given by:

$$
L=\left[\frac{\operatorname{Max}\left(\sigma_{\tau}\right)}{T_{c}}\right]+1
$$

where $\sigma_{\tau}$ is the RMS delay and $T_{c}$ is the chip rate or pulse width depends on the sounding technique. As Eq. 4.7 shows, longer RMS delay spread leads to more channel taps. Also, the channel capacity highly depends on the profile of the channel, tap correlation profile, and the number of taps. 


\section{Propagation in Underground Environments}

\section{Electromagnetic Reverberation}

When a sound wave is emitted into an enclosed space, upon arrival to the listener, it comes with many discrete reflections from the surfaces of the environment. These replicas are mixed and cause the auditory impression of "prolongation" of the sound until the reverberation process "fade away" [153]. Reverberation is a key factor for qualifying the acoustic status in a room because the amount of reverberation generated in the room is directly proportional to the transmitted magnitude and inversely proportional to the sound absorption of the surface. Reverberation occurs when the sounds continually bounce off the reflective surface, e.g. the ceiling, floor, walls and combined all together in the receiver. Conversely, the absorbent surfaces, such as curtains, padded chairs, and even human body can reduce the reverberation phenomenon. Reverberation time is used to quantify the time required for the sound annihilating under a certain threshold in a closed area as shown in Fig. 4.13 [153], which describes the reverberation time it takes for the sound to decay by $60 \mathrm{~dB}$ (i.e. RT60) after the sound source is removed. Practically, it is more common to use the RT30 and RT20 with the thresholds of 30 and $20 \mathrm{~dB}$, respectively.

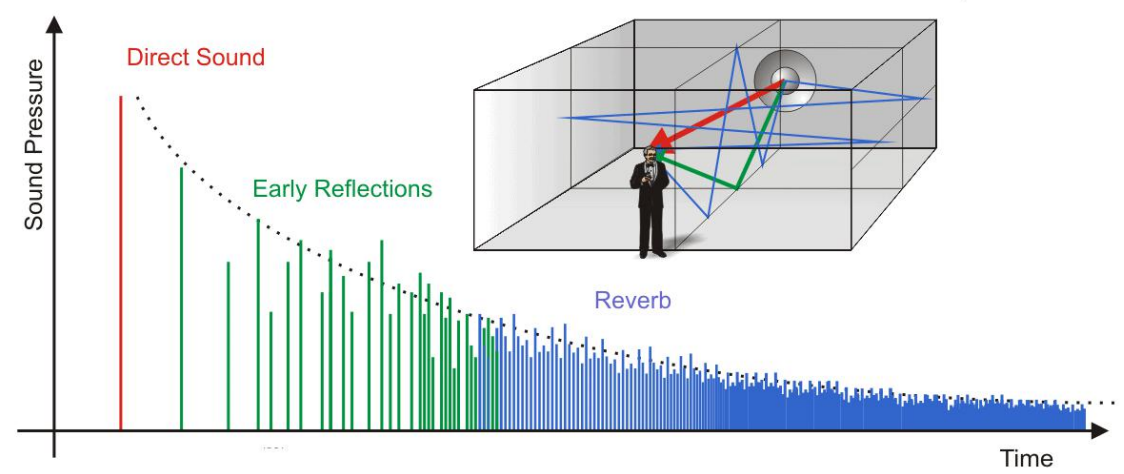

Figure 4.13. The reverberation phenomenon for qualifying the acoustic environment, via: karagioza.com.

Since the wavelengths of acoustic waves and microwaves are typically of the same order in the centimeter range. There is naturally a close resemblance be- 


\section{Propagation in Underground Environments}

tween room acoustics and room electromagnetics [154]. Also, the dimensions of a room are used to much larger than the wavelength, which matches the far-field condition, thus propagated microwaves can be considered as rays in a room. The applicability of methods applied in room acoustics to microwave propagation in a room has been demonstrated in [154].

Room electromagnetic model is a simple model that considers an LOS component (if present) and an exponential PDP decaying with the so-called reverberation time parameter, depending only on the wall area, the volume of the room, and an absorption coefficient. Room electromagnetic theory is interesting, as it does not require complete knowledge of the propagation environment, and it offers an alternative description of diffuse scattering in a room using only simple parameters. The properties of materials are very helpful to improve the simulation-based models. Moreover, the room electromagnetic theory is similar to the special case of S-V model explained in Chapter 2: the single-exponential decay $(\mathrm{L}=1)$ [31]. The single-exponential decay model has a significant first arrival delay path and following with a purely exponential decay multiplied by a lognormally distributed random variable. Also, based on experimental observations of the behavior of the delay-power spectrum and on analogies to models used in room acoustics and electromagnetic fields in cavities. The delay-power spectrum in enclosed space can be modeled as a combination of a dominant component and a reverberant component [155], as simplified in Fig. 4.14.

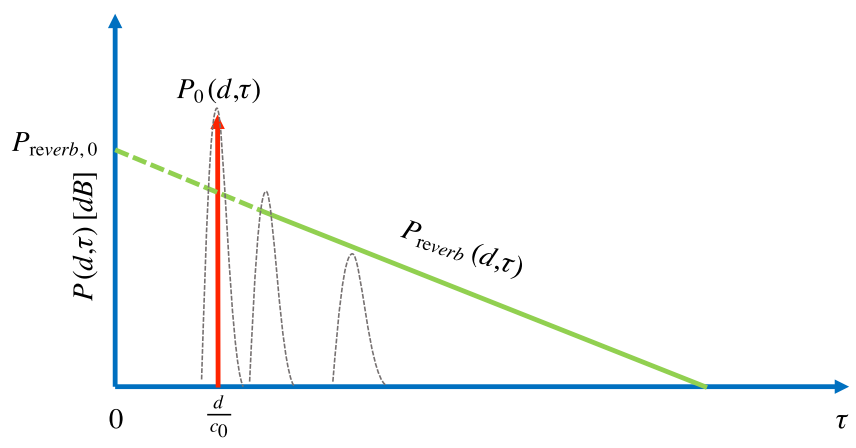

Figure 4.14. The concept of delay power spectrum in reverberation model.

As discussed in Chapter 2, the delay power is term to the square of the channel 


\section{Propagation in Underground Environments}

impulse response with separation distance $d$ between transmitter and receiver as follows:

$$
P(d, \tau)=\mathrm{E}\left[|h(d, \tau)|^{2}\right]
$$

The mean operation $E[\cdot]$ is the expectation of the squared magnitude of the impulse response. As Fig. 4.14 shows, the PDP $P(d, \tau)$ can be derived by the combination of the first arrival component and the reverberant components as follows [156]:

$$
\begin{aligned}
P(d, \tau) & =P_{0}(d, \tau)+P_{\text {reverb }}(d, \tau) \\
& =P_{0,0}\left(\frac{d}{d_{0}}\right)^{n} \delta\left(\tau-\frac{d}{c_{0}}\right)+P_{\text {reverb }, 0} \exp \left(-\frac{\tau}{\tau_{r}}\right) u\left(\tau-\frac{d}{c_{0}}\right)
\end{aligned}
$$

where $P_{0}(d, \tau)$ denotes the first arrival dominant component, which is contributed by the direct path (LOS) or first-order reflection (NLOS). This first arrival dominant component is mainly determined by the separation of transmitter and receiver $d$, which is the first absolute time delay. Hence, it is defined by the large-scale parameters: the reference power $P_{0,0}$ at reference distance $d_{0}$, the PLE $n$, and the velocity of light $c_{0} . \delta(\cdot)$ and $u(\cdot)$ in Eq. 4.9 denote the Dirac delta function and unit step function, respectively. The reverberant components represent the multipath receiving at the receiver side, which is exponentially decayed in the period of reverberation time $\tau_{r}$.

\section{Reverberation Time}

The discrete echoes accompany the direct sound as shown in Fig 4.13, which are not perceived as such due to the time proximity and the quantity. Therefore they could not be differentiated separately but as a whole queue of progressively decreasing signal. Consequently, the reverberation process is divided into two sub-process, named early reflections and reverberation tail. The denser early reflections and with short arrival time indicates a more compact room and vice versa.

As the theory derivation proved in [154], the reverberation model from room 


\section{Propagation in Underground Environments}

acoustics also can be applied into room electromagnetics with similar assumptions on the isotropic diffuse field. The energy density $W$ in a totally diffuse field is given by:

$$
W=4 \pi \frac{I}{c_{0}}
$$

Where $I$ is the ray intensity and $c_{0}$ is the velocity of light. Assuming the volume of the room is uniformly distributed, then the total stored energy of diffuse radiation is $W \times V$. One other thing to note is the energy is partly absorbed by the interior surface of the room. Since ray tube intensity $I$ is independent of direction, the absorbed power $P_{a b}$ is defined by the integration of $I$ over a halfspace as follows:

$$
P_{\mathrm{ab}}=\eta \mathrm{S} \pi I
$$

where $\eta$ is the absorption coefficient, which denotes the fraction of energy absorbed by the surface area $S$. Hence, combining Eq. 4.10 and Eq. 4.11 the input power radiated by the transmitting source $P_{s}$ can be defined as the sum of diffused power and absorbed power, which is given by:

$$
P_{s}=P_{d i f}+P_{a b}=V \frac{d W}{d t}+W \frac{c_{0} \eta \mathrm{S}}{4}
$$

When the radiation source is turned off, Eq. 4.12 becomes to a homogeneous equation. Then the solution is obtained by [154]:

$$
\begin{gathered}
W=W_{0} e^{-t / \tau_{r}} \\
\tau_{r}=\frac{4 V}{c_{0} \eta S}
\end{gathered}
$$

Where $\tau_{r}$ is the electromagnetic reverberation time, which is solely determined by room size and the properties of the absorption surface. Apart from the substitution of sound's speed by the velocity of light, this equation is consistent with the classical Sabine's formula in acoustics [154].

In addition, authors in [156] have introduced the Eyring's formula from room 


\section{Propagation in Underground Environments}

acoustics to provide an alternative solution to define the reverberation time. The Eyring's formula takes into account relatively higher absorption coefficient, and the reverberation time is expressed by:

$$
\tau_{r}=\frac{4 V}{-\ln (1-\eta) c_{0} S}
$$

Unlike the diffuse filed assumption in Sabine's model, Eyring's formula is proposed based on the mirror source theory, which considers a sound field is composed of plane sound waves. These waves lose energy each time when they hit an absorption surface. The fraction of absorbed energy is $(1-\eta)$, where $\eta$ is the surface's absorption coefficient. More details can be found in [157].

Furthermore, indoor electromagnetic reverberation measurements in [158, 159] have proved that the reverberation time can be calculated based on the exponential decay of the PDP's tail. The reverberation time is given by:

$$
\tau_{r}=-\frac{10 \log (e)}{\text { slope }}
$$

where $e$ is the Euler's constant, and slope is the slope of the linear regression of the PDP's tail in log-scale.

To define the PDP's tail, a delay interval $T_{D}$ can be positioned to minimize the influence of the first arrival component and distinguish it with the reverberant components, as shown in Fig. 4.15. In the case of NLOS, since the first arrival component is usually the first order reflection, $T_{D}$ can be equaled to $T_{W}$, which is the width of the transmitted pulse. Whereas for LOS condition, $T_{D}$ plus the maximum propagation time $T_{P}$ along the separation of transmitter and receiver can be selected as the delay interval to obtain the reverberation time. In other words, the delay interval between the first arrival component and reverberant components is given by:

$$
T_{D}=\left\{\begin{array}{lc}
T_{W} & N L O S \\
T_{W}+T_{P} & L O S
\end{array}\right.
$$

Eventually, the slope of the tail can be obtained by the linear regression between the delay after $T_{D}$ and the maximum delay above the threshold, as shown in Fig. 4.15. 


\section{Propagation in Underground Environments}

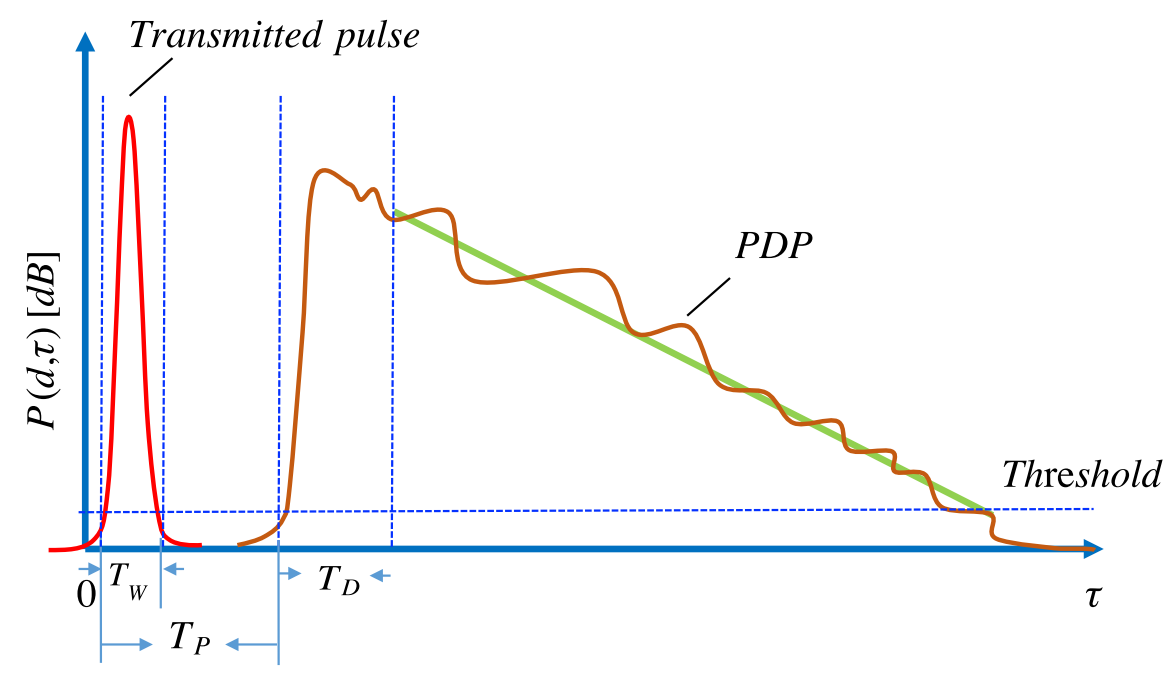

Figure 4.15. Definition of PDP's slope.

Given the above, the reverberation time can be calculated from the PDP, which has a tail exponentially decay with the delay time. Moreover, the corresponding absorption coefficients can be obtained on the basis of reverberation time, when the space volume and the area of absorption surface are available.

\section{Channel Modeling for TEST I}

Based on the TDL model and electromagnetic reverberation theory discussed above, the mean power, RMS delay, and reverberation time of PDPs in TEST I are calculated and summarized in TABLE 4.10.

The first interesting point of TABLE 4.10 is the different performances at two different frequencies. The values of RMS delay spread at $980 \mathrm{MHz}$ over a hundred nanoseconds, which are much longer than these values at $2450 \mathrm{MHz}$ from 21.4 ns to $48.4 \mathrm{~ns}$. This implies the radio waves experienced longer path to arrive the receiver side at $980 \mathrm{MHz}$ than at $2450 \mathrm{MHz}$. Thus, higher frequency yields shorter RMS delay and therefore high data capacity can be reached.

When the head of the train is $-74.5 \mathrm{~m}$ away from transmitting system, i.e. the train is located in the middle of the subway station, the receiving system obtained the shortest RMS delay, no matter at $980 \mathrm{MHz}$ or $2450 \mathrm{MHz}$. This could be explained by the impact of the train body, which significantly narrowed 


\section{Propagation in Underground Environments}

Table 4.10. Train's influences on the mean power, RMS delay and reverberation time in the subway station.

\begin{tabular}{c|c|c|c|c}
\hline \hline Frequency & Tx-Train Separation & Mean Power & RMS delay & Reverberation \\
\hline \multirow{5}{*}{$980 \mathrm{MHz}$} & $-74.5 \mathrm{~m}$ & $-23.2 \mathrm{~dB}$ & $128.8 \mathrm{~ns}$ & $67.3 \mathrm{~ns}$ \\
& $26.5 \mathrm{~m}$ & $-22.8 \mathrm{~dB}$ & $151.0 \mathrm{~ns}$ & $78.8 \mathrm{~ns}$ \\
& $46.5 \mathrm{~m}$ & $-22.5 \mathrm{~dB}$ & $152.6 \mathrm{~ns}$ & $78.8 \mathrm{~ns}$ \\
& $76.5 \mathrm{~m}$ & $-21.5 \mathrm{~dB}$ & $159.2 \mathrm{~ns}$ & $96.8 \mathrm{~ns}$ \\
& $126.5 \mathrm{~m}$ & $-22.2 \mathrm{~dB}$ & $141.2 \mathrm{~ns}$ & $77.6 \mathrm{~ns}$ \\
\hline \hline & $-74.5 \mathrm{~m}$ & $-15.5 \mathrm{~dB}$ & $21.4 \mathrm{~ns}$ & $12.3 \mathrm{~ns}$ \\
& $0 \mathrm{~m}$ & $-14.0 \mathrm{~dB}$ & $27.3 \mathrm{~ns}$ & $17.1 \mathrm{~ns}$ \\
& $10.0 \mathrm{~m}$ & $-16.1 \mathrm{~dB}$ & $27.3 \mathrm{~ns}$ & $16.4 \mathrm{~ns}$ \\
& $16.5 \mathrm{~m}$ & $-18.3 \mathrm{~dB}$ & $48.4 \mathrm{~ns}$ & $29.0 \mathrm{~ns}$ \\
& $21.5 \mathrm{~m}$ & $-16.9 \mathrm{~dB}$ & $43.4 \mathrm{~ns}$ & $25.9 \mathrm{~ns}$ \\
& $31.5 \mathrm{~m}$ & $-16.0 \mathrm{~dB}$ & $42.7 \mathrm{~ns}$ & $25.8 \mathrm{~ns}$ \\
& $46.5 \mathrm{~m}$ & $-18.5 \mathrm{~dB}$ & $39.5 \mathrm{~ns}$ & $21.7 \mathrm{~ns}$ \\
& $126.5 \mathrm{~m}$ & $-18.8 \mathrm{~dB}$ & $37.0 \mathrm{~ns}$ & $19.5 \mathrm{~ns}$ \\
\hline \hline
\end{tabular}

down the size of propagation space. This results in the waves are reflected by the train body, thus can not arrive at the wall on the other side of the track, causes the RMS delay not as long as the train located inside the tunnel. Similar conclusions can be found in $[160,161]$, which states the RMS delay increases as the size of the room increases. Moreover, when the train is stopped near the entrance of the tunnel, A higher RMS delay is obtained as more than $150 \mathrm{~ns}$ at $980 \mathrm{MHz}$ and more than $40 \mathrm{~ns}$ at $2450 \mathrm{MHz}$. Since the train is moved into the tunnel, the space of the station is released. Thus the delay spread becomes longer. It worth noting that, the strong reflections from the steel surface of the train body still exist in this case. Consequently, as the train is located in the deeper tunnel, e.g. the transmitting system and the head of the train are separated to $126.5 \mathrm{~m}$, the impact of the train is suppressed and results in a shorter RMS delay: $141.2 \mathrm{~ns}$ at $980 \mathrm{MHz}$; and $37 \mathrm{~ns}$ at $2450 \mathrm{MHz}$. Note that as far as the train is $50 \mathrm{~m}$ away from the station entrance (Tx-Train Separation: $76.5 \mathrm{~m}$ ), the impact of the train body is still present. While this effect is decreased from $16.5 \mathrm{~m}$, which means the propagations at lower frequencies suffer more and influences from the train body.

Generally, one can compare the parameters between the testing train is present 


\section{Propagation in Underground Environments}

and absent in the station. It can be found that the impact on the received signal from the train body is small with very close RMS delay spread (i.e. same number of channel taps) and mean received power. There is a relatively greater influence created by the train body when the train's head is driving into the station, but it is usually quickly finished in the commercial use. Therefore, the influences from the train body on the propagation in the subway system are limited, especially at lower frequencies.

\section{TEST II: Region-based TDL model}

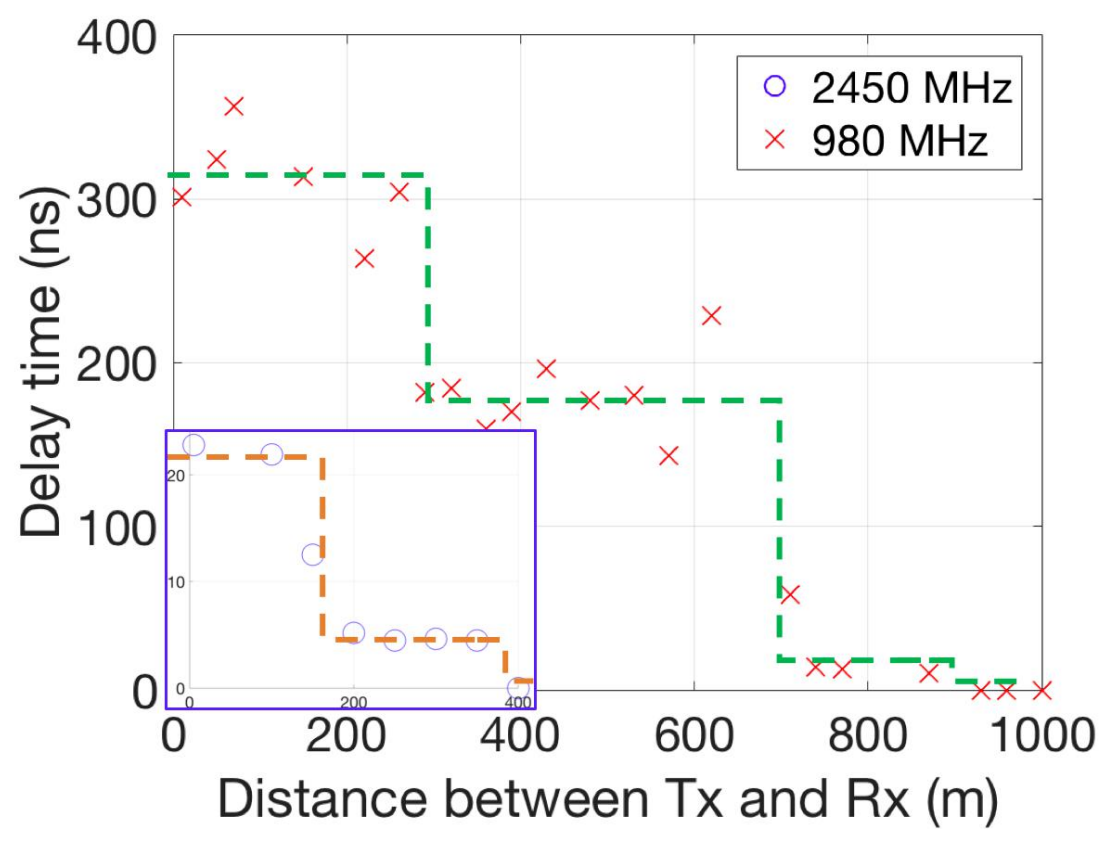

Figure 4.16. RMS delay of TEST II at $980 \mathrm{MHz}$ and $2450 \mathrm{MHz}$.

The RMS delay spread samples along the receiver moving path are collected in Fig. 4.16. As shown in Fig. 4.16, the RMS delay in the station is high, and achieve the peak value when the receiver arrived at the conjunction point of the station and tunnel. This is due to the indoor space between the transmitter and receiver becomes larger since the train driving into the tunnel. Thus a higher RMS delay is obtained. However, as the train moves into the deep tunnel, the 


\section{Propagation in Underground Environments}

effect of the station disappeared gradually. Simultaneously, the filtering effect of the tunnel appears. Therefore the RMS delay decreases with the separation distance between the transmitting system and receiving system increases, These effects from the tunnel are also worth to evaluate. As discussed in [162], radio propagation in a tunnel is mainly affected by the carrier frequency, properties of the tunnel, and the separation between the transmitter and receiver. Furthermore, the different propagation mechanism in different regions can be collected to achieve a complete model in the tunnel. Unlike the narrowband measurements in [162], TEST II provides deeper insight for small-scale characterizations, such as time dispersion and electromagnetic reverberation. Since the RMS delay in Fig. 4.16 presents the stepped shape at both frequencies, the region-based models also can be established as shown in Fig. 4.17.

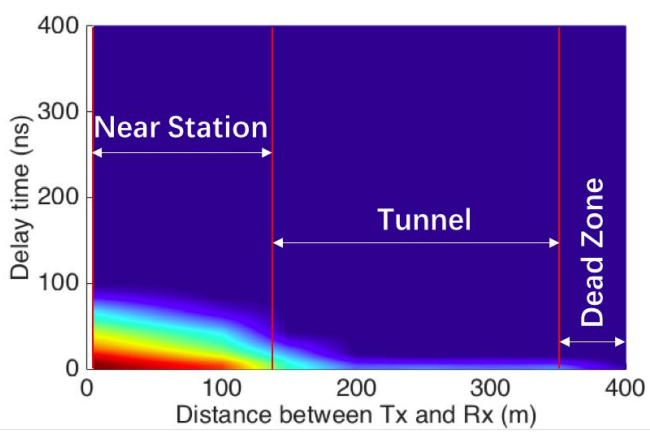

(a)

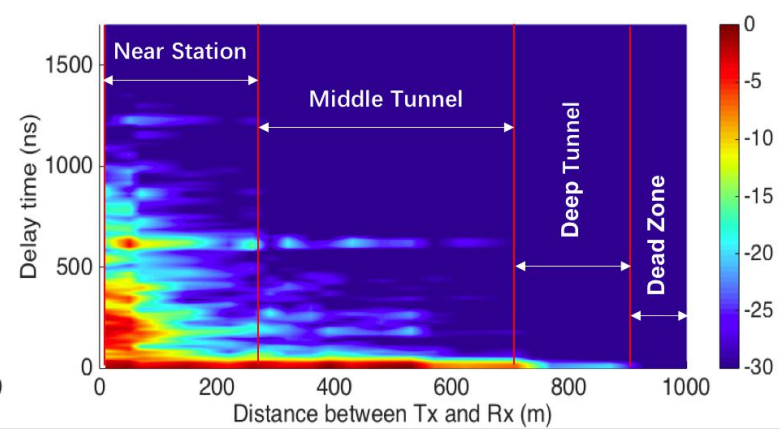

(b)

Figure 4.17. Region division for evaluating the different influences from the different zone in tunnel along track: (a) $2450 \mathrm{MHz}$; (b) $980 \mathrm{MHz}$.

As measurement results show, multipath components at higher frequency decrease faster than at lower frequency. Specifically, the delay spread at $2450 \mathrm{MHz}$ almost vanished after $400 \mathrm{~m}$ in the tunnel, whereas the visible delay spread can be found over $900 \mathrm{~m}$ at $980 \mathrm{MHz}$. Hence, based on the region division in Fig. 4.16 and Fig. 4.17, the key parameters for a region-based TDL model, such as the averaged RMS delay, the number of channel taps are calculated in each region, and listed in Table. 4.11. 
4. Propagation in Underground Environments

Table 4.11. Key parameters for TDL models in different regions.

\begin{tabular}{cccccc}
\hline \hline Region & Name & Distance Range & Excess Delay & RMS Delay & Taps \\
\hline \multicolumn{5}{c}{$980 \mathrm{MHz}$} \\
\hline I & Near Station & $0-275 \mathrm{~m}$ & $1361 \mathrm{~ns}$ & $310.6 \mathrm{~ns}$ & 8 \\
\hline II & Middle Tunnel & $275-725 \mathrm{~m}$ & $746 \mathrm{~ns}$ & $192.5 \mathrm{~ns}$ & 5 \\
\hline III & Deep Tunnel & $725-945 \mathrm{~m}$ & $49 \mathrm{~ns}$ & $64.8 \mathrm{~ns}$ & 2 \\
\hline IV & Dead Zone & $945-1000 \mathrm{~m}$ & $\approx 0 \mathrm{~ns}$ & $\approx 0 \mathrm{~ns}$ & 1 \\
\hline \hline \multicolumn{5}{c}{$2450 \mathrm{MHz}$} \\
\hline I & Near Station & $0-150 \mathrm{~m}$ & $200 \mathrm{~ns}$ & $19.1 \mathrm{~ns}$ & 2 \\
\hline II & Tunnel & $150-350 \mathrm{~m}$ & $36 \mathrm{~ns}$ & $4.7 \mathrm{~ns}$ & 1 \\
\hline III & Dead Zone & $350-400 \mathrm{~m}$ & $\approx 0 \mathrm{~ns}$ & $\approx 0 \mathrm{~ns}$ & 1 \\
\hline \hline
\end{tabular}

Note: (1) The "Near Station" Regions include part of station about 55m long;

(2) The " $0 \mathrm{~m}$ " stands for the middle point of the station's length.

\section{TEST II: Absorption Coefficient \& Q factor}

The quality factor (Q) is used to describing the capacity of electromagnetic energy stored in a resonant system [159]. The underground system, such as subway environment is almost sealed. Therefore, the subway station and tunnel can be roughly regarded as a cavity resonator that can store the energy radiated from the transmitting source in the enclosed space. Moreover, the Q can be obtained by the power ratio method in the frequency domain and based on the decay time method in time domain. Authors in $[163,164]$ have concluded that decay time method has achieved a better estimation on Q. Hence, the reverberation time obtained by formulas or PDP curves provides a feasible way to estimate the Q. Simultaneously, room electromagnetics theory is equally applicable to the electromagnetic wave propagate in the underground system. The $\mathrm{Q}$ factor reveals the channel capacity and the electromagnetic energy store in enclosed space, which is frequency-dependent and defined by:

$$
Q=2 \pi f \tau_{r}
$$

Here $\tau_{r}$ denotes the time constant of the free energy relaxation of the cavity resonator's field (exponential decay) if the input power is switched off, i.e. reverberation time. Eq. 4.14 and Eq. 4.18 describe the original definitions of the 


\section{Propagation in Underground Environments}

reverberation time $\tau_{r}$ and $Q$ factor in a cavity resonator that has a volume of $V$, and the surface is covered by the materials with the absorption coefficient $\eta$ and the absorption area of $S . c_{0}$ denotes the velocity of light. In subway environments, the subway station is mainly constructed by the steel structures. Yet, the inner wall of the tunnel is constituted by the smooth concrete. Thus the absorption coefficient $\eta$ in these two scenarios can be different. Moreover, since the dimensions of the subway station and tunnel are easy to obtain, the $\mathrm{Q}$ can be described as follows:

$$
Q=Q_{\text {Density }} \times \kappa \text {, where } \kappa=\frac{V}{S}
$$

where $Q_{\text {Density }}$ denotes the density of $\mathrm{Q}$ value in a specific space coefficient $\kappa$, which is the ratio of the volume $V$ to the absorption surface area $S$. Based on the dimensions of the subway station and the cross sections of tunnel marked in Fig. 4.5 and Fig. 4.6, the $\kappa$ values in station and tunnel can be calculated as two different constants: $\kappa_{s} \approx 2.8$ in the subway station and $\kappa_{t} \approx 1.8$ in the tunnel.

Consequently, the values of the reverberation time $\tau_{r}(d)$ and $Q(d)$ along the train's moving path from the station into the tunnel can be obtained based on Eq. 4.16 and Eq. 4.18 at every position. Furthermore, the absorption coefficient $\eta(d)$ and $Q_{\text {Density }}(d)$ based on the Sabine's formula and Eyring's formula can be defined as the functions of distance between transmitter and receiver as follows:

$$
\begin{gathered}
\eta_{S a b}(d)=\frac{4 \kappa}{c_{0} \tau_{r}(d)} \\
\eta_{E y r}(d)=1-\exp \left[-\frac{4 \kappa}{c_{0} \tau_{r}(d)}\right] \\
Q_{\text {Density }}(d)=\frac{Q(d)}{\kappa}=\frac{2 \pi f \tau(d)}{\kappa}=\frac{8 \pi f}{c_{0} \eta(d) \kappa}
\end{gathered}
$$

As the key parameters for electromagnetic reverberation model listed in Table. 4.12, the reverberation time and $Q_{\text {Density }}$ have the similar downward trend with the RMS delay and Taps number as the train moves from the station into the tunnel. Moreover, since the absorption coefficients $\eta_{S a b}$ and $\eta_{E y r}$ has the relationship of $\eta_{E y r}=1-\exp \left(-\eta_{S a b}\right)$, the Sabine's model and Eyring's model can be easily transformed. 
4. Propagation in Underground Environments

Table 4.12. Key parameters for reverberation models in different regions.

\begin{tabular}{cccccc}
\hline \hline Region Index & Name & Avg. $\tau_{r}$ & Avg. $\eta_{\text {Sab }}$ & Avg. $\eta_{\text {Eyr }}$ & Avg. $Q_{\text {Density }}$ \\
\hline \multicolumn{5}{c}{$980 \mathrm{MHz}$} \\
\hline I & Near Station & $196.7 \mathrm{~ns}$ & 0.19 & 0.17 & 432.6 \\
\hline II & Middle Tunnel & $111.3 \mathrm{~ns}$ & 0.22 & 0.19 & 380.8 \\
\hline III & Deep Tunnel & $42.7 \mathrm{~ns}$ & 0.56 & 0.43 & 146.1 \\
\hline \hline \multicolumn{7}{c}{$2450 \mathrm{MHz}$} \\
\hline I & Near Station & $14.7 \mathrm{~ns}$ & 2.53 & 0.92 & 81.0 \\
\hline II & Tunnel & $10.9 \mathrm{~ns}$ & 2.19 & 0.89 & 93.2 \\
\hline \hline
\end{tabular}

\section{TEST II: Transition Distance}

In addition, Fig. 4.18 shows the $Q$ values vary with the separations between transmitter and receiver at $980 \mathrm{MHz}$ and $2450 \mathrm{MHz}$. Since the start point of the train's moving is the middle of the subway station, $\mathrm{Q}$ reaches a high value in the first $56 \mathrm{~m}: \approx 1200$ at $980 \mathrm{MHz}$ and $\approx 220$ at $2450 \mathrm{MHz}$. As the train moves into the tunnel, these high $\mathrm{Q}$ values do not decrease rapidly, even space becomes smaller, and the surface is replaced by the concrete greater absorption coefficient for electromagnetic waves. This indicates the impact of the subway station still exists over a certain distance. When the distance between transmitter and receiver reaches more than $200 \mathrm{~m}$, the confined tunnel results in the filtering effect on the wave reverberations. Thus the reverberation time sharply decreases along the distance until $800 \mathrm{~m}$ at $980 \mathrm{MHz}$ and 400m at $2450 \mathrm{MHz}$. After this area, the $\mathrm{Q}$ values almost equal to 0 , thus almost no reverberation presents at two frequencies. The transition distance is thus defined as the distance from the position in the tunnel with long reverberation time and high value to a region with a very short reverberation time and low $\mathrm{Q}$ factor, impaired with the train going deeper into the tunnel. To clarify this transition distance, we propose the simplified models of the Q versus distance as shown is Fig. 4.19.

After the station region $L_{s}$, The transition distance $d_{T}$ is roughly considered to consist of two parts: the part affected by the influence from the subway station to the tunnel denoted by $d_{1}$, which leads to the maintenance of the high $\mathrm{Q}$ factor; the sharp decreasing region that the reverberation decreases from high level to 


\section{Propagation in Underground Environments}

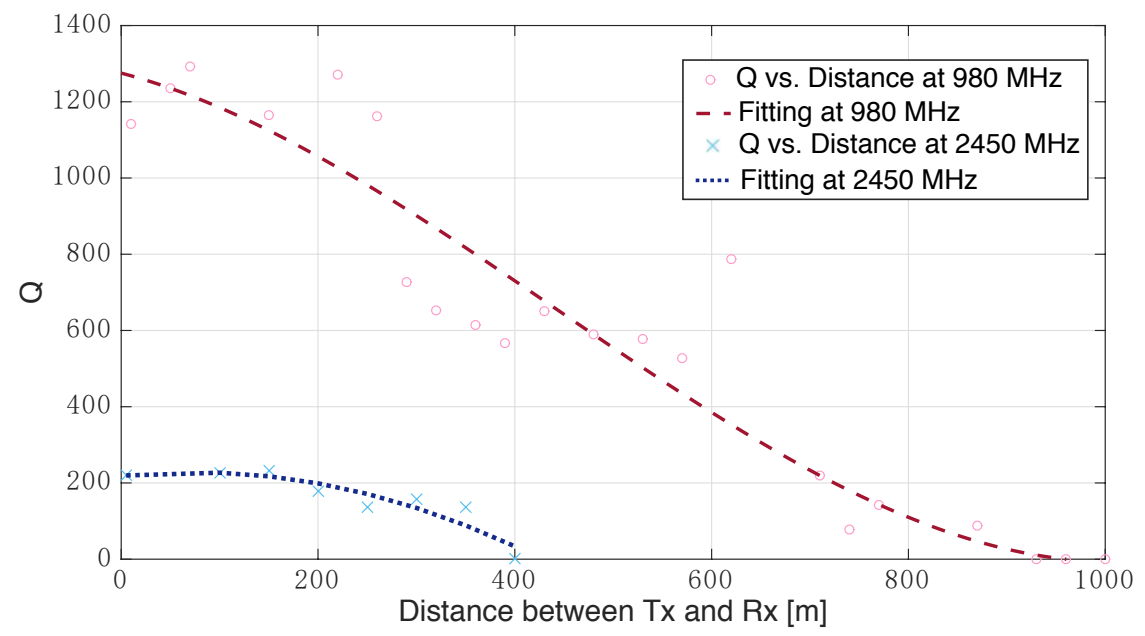

Figure 4.18. Q-factor variations and fittings versus distance at $980 \mathrm{MHz}$ and 2450 $\mathrm{MHz}$.

the very low level, described by $d_{2}$. Thus, the transition distance is represented by:

$$
d_{t}=d_{1}+d_{2}
$$

Based on our measurement results, $d_{1}$ at both frequencies are close to $90 \mathrm{~m}$, which can be estimated by the dimension parameters of the subway station $L_{s}$, $\kappa_{s}$, and $\kappa_{t} . L_{s}$ is the distance between the transmitter and the farthest wall. $\kappa_{s}$ and $\kappa_{t}$ denote the ratio of volume to the area of absorption surface $V / S$ in the subway station and tunnel, respectively. Moreover, the other part of the $d_{t}$ can be obtained by the $Q_{s}$ and slope $s$ of the simplified linear transition model in Fig. 4.19. It worth noting the slope $s$ is inversely proportional to the frequency. If the measurement results as the example values are introduced in the model, the transition distance can be roughly estimated by:

$$
d_{t} \approx \frac{L_{s} \kappa_{s}}{\kappa_{t}}+\frac{Q_{s}}{-s_{0}} \times \frac{f}{f_{0}}
$$

Where $s_{0}$ and $f_{0}$ are the reference slope and frequency. Base on our measurement results, $s_{0} \times f_{0}$ at two frequencies in $\mathrm{GHz}$ are equal to -1.96 , which can be considered as a example value. Therefore the transition distance can be redefined 


\section{Propagation in Underground Environments}

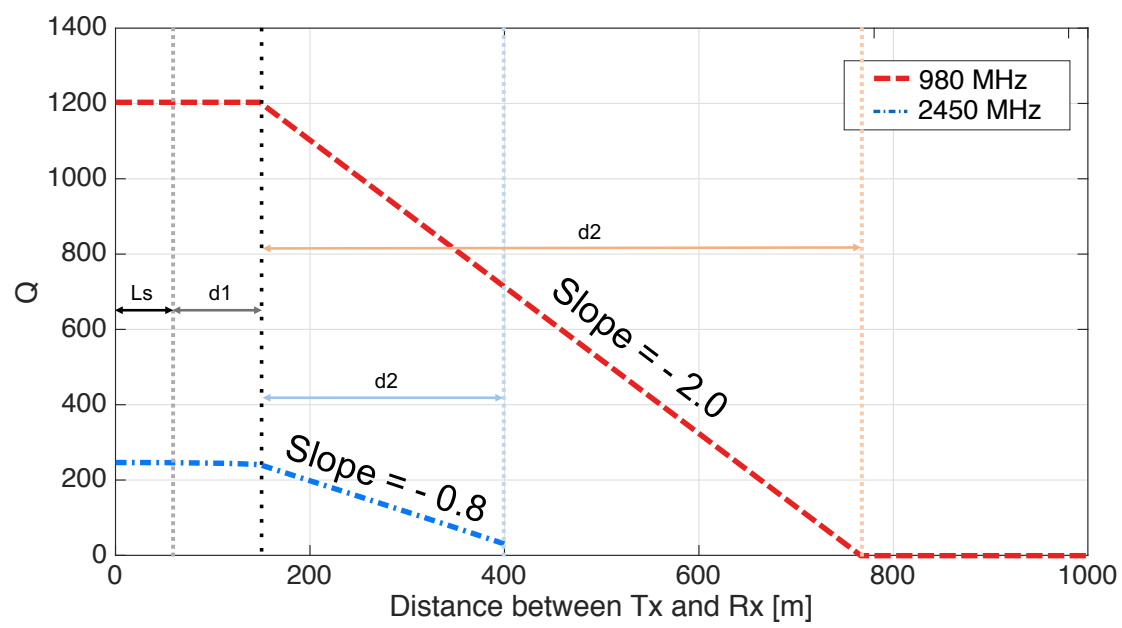

Figure 4.19. Transition model base on the Q-factor variations at $980 \mathrm{MHz}$ and $2450 \mathrm{MHz}$.

based on Eq. (4.16) and Eq. (4.18) and plugging the example value -1.96 in Eq. 4.23 as follow:

$$
d_{t} \approx \frac{L_{s} \kappa_{s}}{\kappa_{t}}+\frac{Q_{s}}{1.96} \times f=\left(\frac{L_{S}}{\kappa_{t}}+\frac{8 \pi f^{2}}{1.96 c_{0} \eta_{s}}\right) \times \kappa_{s}
$$

Based on Eq. 4.24, if the dimension parameters of station and cross section of the tunnel are supplied, the transition distance and absorption coefficient $\eta_{s}$ can be estimated.

The model combines with the measurements can provide detailed information about the behavior of wideband communications in subway system and helpful for determining the optimized antenna configurations. Actually, in most subways antennas are usually installed in the stations, nevertheless installing them inside the tunnels (after $d_{1}$ ) is expected to highly reduce the multipath effects. Also, $2 \times 2$ MIMO configuration is recommended to mount one antenna in the station and the other inside the tunnel, with different polarizations. With these settings, the channels can be highly uncorrelated. 


\section{Propagation in Underground Environments}

\subsection{Discussion}

In this chapter, both narrowband and wideband measurements conducted in subway systems are presented for describing propagation behaviors in underground environments. Based on the large-scale fading characteristics and comparisons in Part I, and time dispersion analysis in Part II, some useful discussions can be addressed as follows:

i. Path loss: PLEs in the semicircular $(3.36 \sim 5.50)$ are generally larger than the values in the arched tunnel $(0.94 \sim 3.73)$, which implies the transmitters (AP/BTS) in the curved traditional arched tunnels can be deployed more sparsely than in the modern semicircular tunnels. Since most of the obtained PLE values are greater than 2, the tunnel curve is considered to reduce the waveguiding effect and results in the extra loss with respect to the straight tunnel. Moreover, the sharper the tunnel curve is, the greater the path loss is. Besides, radio signals in horizontal polarization and at higher frequencies are more sensitive to the tunnel curve. Note that PLE values in the curved tunnel are larger than in the similar standard scenarios of WINNER models.

ii. Shadow fading: the measured shadow fading is well fitted by the log-normal distribution. The standard deviations of the shadow fading in curved tunnel show curved subway tunnel results in more severe shadow fading than in the other railway scenarios, such as railway station, cutting, and viaduct. In addition, the standard deviations of shadow fading in the curved tunnel are close to the values in the outdoor-to-indoor scenarios of WINNER. Thus, the existing models in standard scenarios are insufficient to represent the propagation in the curved tunnel.

iii. Autocorrelation and decorrelation distance: through the comparative analysis, the 802.16J model performs more suitable for the curved arched subway tunnels. Then, the several meters decorrelation distances are much shorter than this value in the urban macro environments or other outdoor rail traffic scenarios. Therefore, the shadow fading component along the distance varies faster in the curved tunnel than in the open space, which is worthy of additional concern in the communication system design. 
iv. Cross-correlation of shadow fading: the signals at different frequencies or in different types of tunnels are uncorrelated. Therefore, the frequency diversity can be applied to ensure a reliable communication in subway systems for emergency cases. Moreover, various communication systems in various frequency bands are expected to work simultaneously in the same curved tunnel without severe interference. Note that the shadow fading of received signal in different types of tunnels is very different. Thus the network planning should be conducted independently in various kinds of curved tunnels.

v. Influence from the station \& train: the wideband signal can be affected by the train parked in the station. The train's body compresses the propagation inside the station and results in a short time delay than in the empty station. When the train is located near the entrance of the tunnel to the station, longer delay spread is achieved due to the reflection on the train's head. These settings lead to the variations of the time dispersion at the receiver side, especially at a higher frequency. Nevertheless, these variations are relatively slight, which can be ignored in the actual operation. As the train stopping at different positions in the subway station and tunnel, the values of RMS delay and reverberation time at the receiver side are very close at the same frequency. Note that the CIRs of the radio waves at higher frequency have shorter RMS delay spread and reverberation time.

vi. Influence from the tunnel: In the case of the receiver attached to the train, the influence from the tunnel on the propagation can be observed. As the receiver moves from the subway station to the tunnel, the MPCs contributed by the station are gradually attenuated by the tunnel. The RMS delay at 980 $\mathrm{MHz}$ decreases from about $310 \mathrm{~ns}$ inside the station to $0 \mathrm{~ns}$ after $940 \mathrm{~m}$ away from the transmitter inside the tunnel. Yet, this decreasing process begins with a much lower RMS delay and is finished much faster at $2150 \mathrm{MHz}$, the MPCs contributed by the station are almost faded away after $350 \mathrm{~m}$ inside the tunnel. Base on the ladder-like trend of RMS delay, the region-based TDL models can be established, which characterize the channel along the distance with different regions. The average time delay and the number of channel taps are calculated for each region. Besides, the reverberation time 
and corresponding absorption coefficients are provided for each region on the basis of electromagnetic reverberation theory. Also, the frequent dependent parameter $\mathrm{Q}$ is calculated along the distance. Based on the change of $\mathrm{Q}$ along the distance, a transition distance is defined from the position with high $\mathrm{Q}$ to the position with very low $Q$. The estimation model for the transition distance is of interest for wireless communication system design and updating in subway systems. 


\section{Chapter 5}

\section{Propagation in High Mobility Scenarios}

The radio coverage and transmission rate of most existing wireless communication systems are significantly limited by the high mobility scenarios. Hence, development of wireless communication system for high mobility environment has generated a lot of public interest in recent years. In addition to known HighSpeed Railway (HSR) system, there appear many new application scenarios such as highway vehicular communications systems and Low Altitude Flying Object (LAFO) systems [165]. The maximum train moving speed of HSR trialed by the French National Rail Corporation has reached nearly 575 km/h [166]. Whereas the relative speed between the transmitter and receiver of LAFO system can even higher than $1000 \mathrm{~km} / \mathrm{h}$. Come to the age of $5 \mathrm{G}$, the research on wireless signal propagation faces new tasks: How to provide a high capacity and speedy data transmission in a high mobility scenario? Before answering this question, a series of special challenges have to be addressed due to the increasing of relative speed. An essential feature of the high mobility scenarios is the fast time-variation of the fading channel caused by the high relative speed with a large Doppler spread. Furthermore, Doppler spreads are also time-varying and then result in a non-

stationary fading channel in the rapidly changing scattering environment. The Doppler shift causes the mismatch between the frequency of the oscillators at transmitter and receiver, which is the so-called Carrier Frequency Offset (CFO). 
CFO can significantly affect the orthogonality among the subcarriers and produces the Inter-Carrier Interference (ICI) in OFDM systems. Note that larger Doppler spread means a high Doppler diversity order, which can be exploited to improve system performance. In the meantime, as the speed increases, the common assumption of perfect Channel State Information (CSI) adopted for the low mobility systems is no longer applies. Thus, the channel estimation errors increase and led to added requirements for system design. Therefore, a tradeoff between Doppler diversity and channel estimation errors has to be considered in the system design. Additionally, it is foreseeable that the handover procedure in high mobility scenarios can be frequent and fast, due to the high speed of movement. Thus, new handover algorithms have to be developed to reduce possible handover failures in a shorter handover time.

All of these challenges can greatly affect the design and development of next generation communication systems in high mobility scenarios. On the one hand, the fast movement of the wireless terminals seriously degrades the system performance. On the other hand, high mobility also provides some unique opportunities that can benefit system designs, on the premise that the propagation channel in high mobility is comprehensively understood. For this purpose, the channel measurement and modeling in these environments is of importance.

There have been many valuable works for channel measurement and modeling in high mobility environments. For example, Vehicle-to-Vehicle (V2V) channel measurements in $5 \mathrm{GHz}$ band $[167,46]$ and simulation [168] reveal the severe fading and statistical non-stationarity in V2V channel models. Besides, the Air-toGround (AG) communication channel has been reviewed in [169], which states the existing AG channel measurement is insufficient because AG channel is different with the terrestrial cellular or indoor channel. The AG channel has to be comprehensively characterized by the mean of experimental investigation. The AG channel measurements by an 802.11a-based small quadrotor (Unmanned Aerial Vehicle) UAV have been conducted with different height in [170], which characterized the impact of UAV 's yaw, height, and distance on the AG channel. The example wideband measurements can be found in [171], which parameterized not only the path loss but also the channel dispersion.

The most-watched high mobility application for wireless propagation is HSR, 
which is widely treated as the fundamental solution for the improvement of the public transportation situations of countries and cities all over the world. The existing global system for mobile for rail (GSM-R) standard supports a maximum data rate up to $200 \mathrm{kbps}$, whereas this narrowband data only serve for train control signals. The fourth generation $(4 \mathrm{G})$ Long Term Evolution for Railway (LTE-R), is expected to provide not only optimized services for train control but also the high-quality internet access for passengers in the future. Several propagation measurement campaigns have been conducted in different HSR scenarios for the wireless channels, e.g., in viaducts [172, 173, 35], hilly terrains $[174,175]$ cuttings [176, 177, 178], train stations [179, 180], and tunnels [181, 182] scenarios. The empirical results analysis and statistical modeling in large-scale and smallscale have been conducted based on these measurements. The TDL models for hilly terrain, viaduct, and plain scenarios on HSR are presented in [183]. In [184], the authors propose an algorithm for joint channel estimation and Doppler frequency offset estimation based on Ricean channel model. Deterministic models based on ray-tracing in HSR can be found in [185, 186].

However, most of the studies above are mainly confined in an isolated scenario. Whereas various scenarios are continuous for wireless communication systems in the real HSR environment. In the meantime, the receiver with high velocity experiences various regions over a very short time, and these regions with different topographies cannot be separated from the view of the receiver. Then the severe fading frequently occurs in these composite regions. The existing research has ignored the details about the transition from one scenario to another as well as small-scale parameters in Doppler domain. Hence, the non-stationarity of rapidly time-varying channels in the composite scenario of HSR requires further investigation.

The first part of this chapter presents the wideband measurements in an HSR station and the time dispersion analysis in both LOS and NLOS conditions. The wideband measurements have also carried out in a composite scenario that composes of a tunnel portal, a viaduct, and two cuttings. The PDPs in this composite regions are presented and analyzed at $950 \mathrm{MHz}$ and $2150 \mathrm{MHz}$. Also, the channel non-stationarity characteristics in these composite regions are investigated by ray tracing simulations. 
Table 5.1. Channel sounder setups

\begin{tabular}{l|c}
\hline \hline Item & Description \\
\hline Frequency & $950 \mathrm{MHz} / 2150 \mathrm{MHz}$ \\
\hline Transmitting power & $35 \mathrm{dBm}$ \\
\hline Pulse width/ period & $30 \mathrm{~ns} / 1000 \mathrm{~ns}$ \\
\hline IF Bandwidth & $100 \mathrm{MHz}$ \\
\hline Noise figure & $3 \mathrm{~dB}$ \\
\hline Sensitivity & $-100 \mathrm{dBm}$ \\
\hline \hline
\end{tabular}

\subsection{Hardware Systems}

The portable channel sounder as introduced in Section 3.2.2 is employed as the systematic hardware system to measure the behaviors of wireless signals in HSR environment. As the wideband test, the channel sounder transmitter generates a series of periodic narrow pulses to stimulate the wireless channel in HSR. The distorted and faded signal is then received by the channel sounder receiver. After the envelope detection, the demodulated signal is sent to the digital oscilloscope, which allows capturing, digitizing, and storing the power delay profile in real time. In order to meet the requirements of high-speed sampling, Keysight Ininiium MSO9104A oscilloscope that offers bandwidths up to $1 \mathrm{GHz}$ is adopted at the receiver side. The high-performance scope channels with responsive deep memory ensure superior viewing of signals under test. The detailed configurations of the portable channel sounder system are listed in Table 5.1.

Moreover, the channel sounder transmitter is equipped with the L-Com HG727 14P-090 panel directional antenna with $90^{\circ}$ horizontal and $17^{\circ}$ vertical beamwidth. The channel sounder receiver is attached to the L-Com HG72107U omnidirectional antenna with 3-meter height. Note both the transmitting and receiving antennas are vertical polarized. More details can be found in Table. 5.2. The antenna photos and radiation patterns can be found in Appendix: Fig. 3(a) and Fig. 3(b). 
5. Propagation in High Mobility Scenarios

Table 5.2. Antenna informations

\begin{tabular}{l|c|c}
\hline \hline Item & Tx Antenna & Rx Antenna \\
\hline Frequency Range & $698-960 / 1710-2700 \mathrm{MHz}$ & $698-960 / 1710-2700 \mathrm{MHz}$ \\
\hline Gain & $13 / 14 \mathrm{dBi}$ & $4 / 7 \mathrm{dBi}$ \\
\hline Vertical Beamwidth & $17^{\circ}$ & $55^{\circ} 28^{\circ}$ \\
\hline Horizontal Beamwidth & $90^{\circ}$ & $360^{\circ}$ \\
\hline Polarization & Vertical & Vertical \\
\hline Front to Back Ratio & $\geq 23 \mathrm{~dB}$ & - \\
\hline Height & $3 \mathrm{~m}$ & $1.2 \mathrm{~m}$ \\
\hline Dimensions & $1568^{*} 320^{*} 160 \mathrm{~mm}$ & Length: $1.02 \mathrm{~m}$ \\
\hline \hline
\end{tabular}

\subsection{Case I: HSR Station}

The train station is one of typical HSR scenarios. Channel characterization in this scenarios has mostly focused on the large-scale characteristics, such as path loss, shadow fading, or the correlation properties [179, 180, 187]. Yet, small-scale features such as time dispersion by the mean of wideband measurements, are rarely studied. The newly-built HSR routes are distinguished from conventional lines, by gentler curves, shallower grooves, and wider tunnels [188]. Also, the modern HSR stations are designed to a gigantic multifunctional complex that usually built largely to provide the additional service and meet the greater requirement of passenger capacity. However, these big buildings present new challenges. For example, with the conventional base station position, there can be a Non-Lineof-Sight (NLOS) scenario on the platform generated by the blocking of the new station. This motivates the wide measurements and channel characterization in both LOS and NLOS regions on the platform.

\subsubsection{Measurement Scenarios and Configurations}

The wideband measurement has been carried out in the "TaiYuan South" railway station, Shan Xi, China as shown in Fig. 5.1. This HSR station has been under construction with a large station hall close to the station platform. As the yellow circle marked in Fig. 5.1, the transmitting system has been set in the open area next to the construction site, which is a typical position that the commercial base 


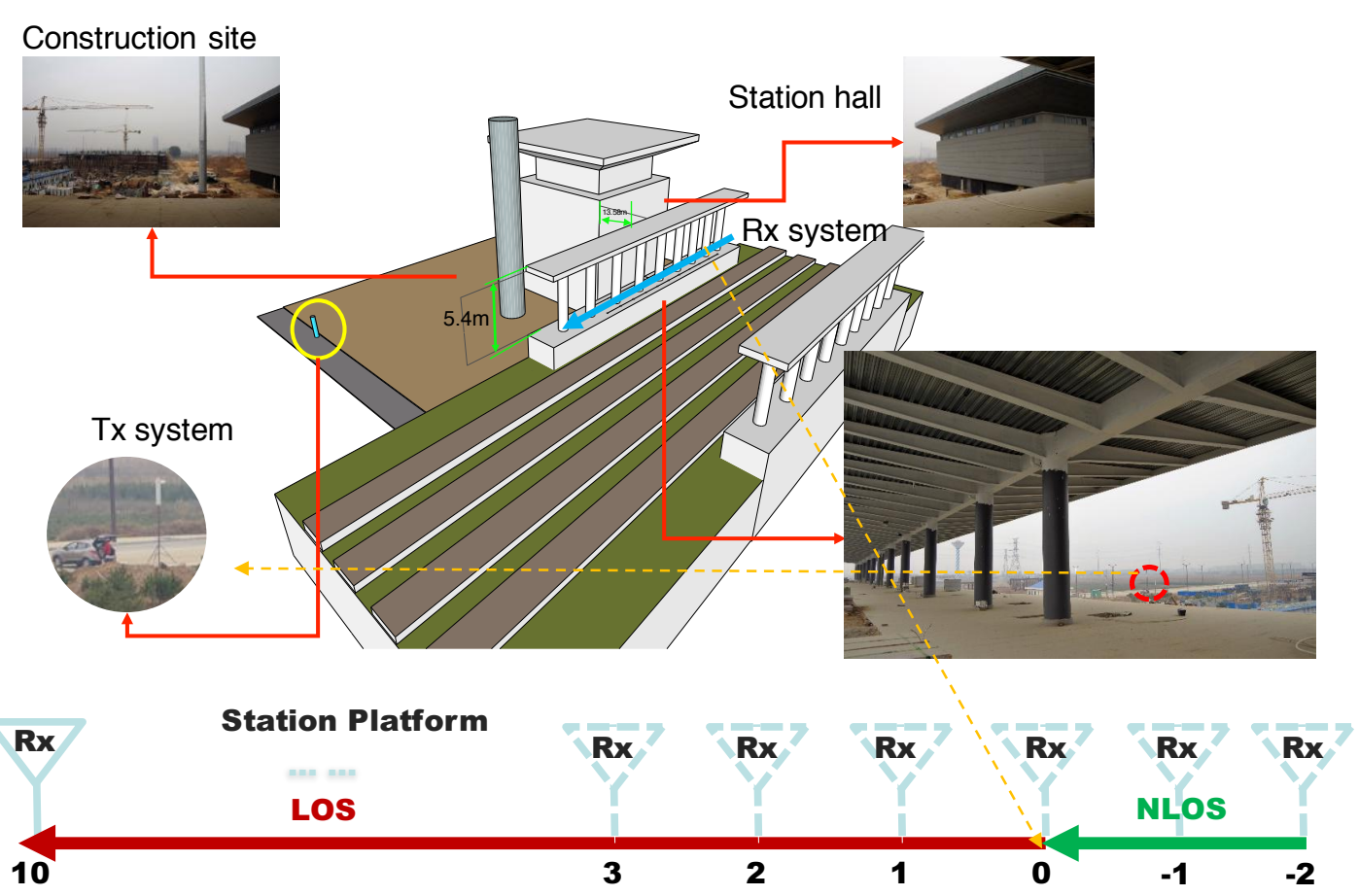

Figure 5.1. Measurement scenarios and configurations in the HSR station.

station located. Moreover, the station platform is shaded by the steel roof. At the receiver side, the receiving system has been arranged to move on the platform passage that close to the station hall and transmitting system. In this case, two different regions can be easily divided for propagation: part of the passage that shaded by the station hall has been an NLOS situation towards to the transmitter, and receiver in the rest part of the passage that has a direct path (LOS) to the transmitter.

To evaluate the difference between the propagation characteristics in LOS and NLOS, the receiving system has been placed in 13 test points that moving on the passage near to the station side, from the NLOS (green line) area to LOS (red line) area as illustrated in Fig. 5.1. There have been 2 points located in the NLOS region, and rest have been placed in the LOS region. Moreover, the first LOS point has been marked to 0 , and the test points in NLOS region have been marked as negative numbers. Afterward, the PDPs on each test point have been 
collected and recorded for the off-line process. For comparison, measurements have been carried out at two carrier frequencies: $950 \mathrm{MHz}$ and $2150 \mathrm{MHz}$, in both LOS and NLOS regions.

\subsubsection{Measurement Results Analysis}

In HSR station scenario, both the transmitter and receiver have many reflectors in their vicinities. More MPCs can be expected due to the complex surrounding.

Fig. 5.2 shows the example PDPs in LOS and NLOS regions at $950 \mathrm{MHz}$ and $2150 \mathrm{MHz}$, respectively. The PDPs in LOS region has a dominant direct path as expected, particularly at $2150 \mathrm{MHz}$. PDP in LOS region at $950 \mathrm{MHz}$ presents some short delay less than $400 \mathrm{~ns}$, whereas propagation in NLOS regions observes longer excess delay beyond 600 ns and fainter dominant path. Similar situations occur at $2150 \mathrm{MHz}$, where almost no obvious delay spread can be found in the LOS region. Also, the shorter time delay and fainter dominant component are presented in NLOS situation at $2150 \mathrm{MHz}$, compared to the PDPs at $950 \mathrm{MHz}$. This is because the radio wave at higher frequency suffers larger attenuation along the propagation paths.
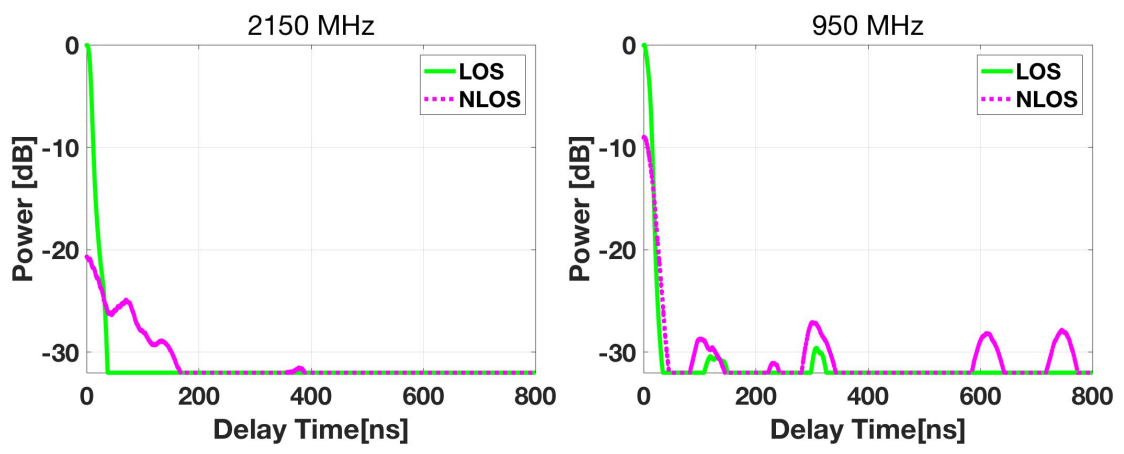

Figure 5.2. The PDPs in LOS and NLOS regions at two frequencies.

Furthermore, the comprehensive measurement results are shown in Fig. 5.3, which presents the received power versus time delay in all test points at $950 \mathrm{MHz}$ and $2150 \mathrm{MHz}$. It can be observed that almost all the test points in LOS region present shorter delay spread at $2150 \mathrm{MHz}$ than at $950 \mathrm{MHz}$. Also, propagation in NLOS region show more MPCs at $950 \mathrm{MHz}$ than at $2150 \mathrm{MHz}$. In short, radio 


\section{Propagation in High Mobility Scenarios}

propagation in HSR station at $2150 \mathrm{MHz}$ suffers less multipath effects than at $950 \mathrm{MHz}$.
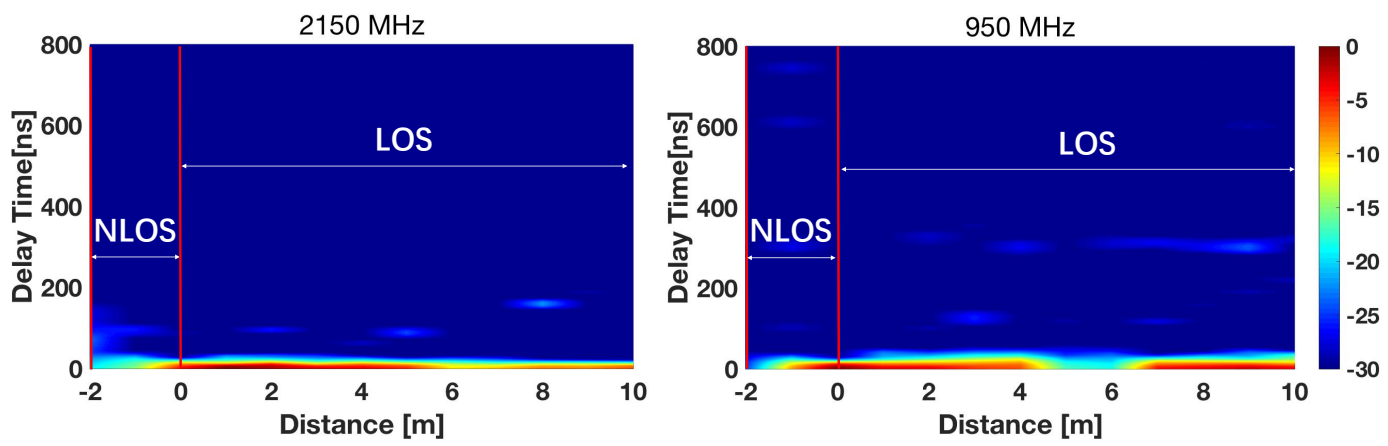

Figure 5.3. The received power versus time delay and test points at two frequencies.

For qualitatively revealing the channel characterizations in LOS and NLOS regions at two frequencies, the RMS delay spread and the number of channel taps are calculated by Eq. 4.6 and Eq. 4.7. The mean parameters are collected and listed in Table. 5.3. The experimental results show propagation at higher frequency is less susceptible to the effects of the scatterers in the complex surrounding of HSR station. Hence, the optimal choice of the carrier frequency for propagation in HSR station is $2150 \mathrm{MHz}$, with respect to $950 \mathrm{MHz}$. To mitigate the large delay spread in the NLOS region of the platform, a repeater or AP should be set.

Table 5.3. Parameters of time dispersion at two frequencies

\begin{tabular}{c|c|c|c|c}
\hline \hline Region & \multicolumn{2}{|c|}{ NLOS } & \multicolumn{2}{c}{ LOS } \\
\hline Frequency & $950 \mathrm{MHz}$ & $2150 \mathrm{MHz}$ & $950 \mathrm{MHz}$ & $2150 \mathrm{MHz}$ \\
\hline Mean Normalized Power & $-17 \mathrm{~dB}$ & $-18 \mathrm{~dB}$ & $-6 \mathrm{~dB}$ & $-6 \mathrm{~dB}$ \\
\hline Average Excess Delay & $430.0 \mathrm{~ns}$ & $128.8 \mathrm{~ns}$ & $424.4 \mathrm{~ns}$ & $55.1 \mathrm{~ns}$ \\
\hline Average RMS Delay Spread & $148.3 \mathrm{~ns}$ & $40.2 \mathrm{~ns}$ & $138.7 \mathrm{~ns}$ & $18.2 \mathrm{~ns}$ \\
\hline Average Number of Taps & 6 & 2 & 5 & 1 \\
\hline \hline
\end{tabular}




\subsection{Case II: HSR Composite Scenario}

Cuttings are widely used in rail construction on the uneven ground to pave the way for high-speed train cut through large obstacles such as hills. A regular deep cutting, where steep walls on both sides of the rails have almost the same depths and slopes. The cutting structure creates a large container which can accommodate lots of reflection and scattering components. Viaducts with a height of $10 \mathrm{~m}$ to $30 \mathrm{~m}$ are also used in HSR to ensure flatness of the rails and thus enable the high speeds of the train. The viaduct raises the antennas, creates a relatively clear LOS channel, and reduces the number of the scatterers that can affect the fading. Therefore, cutting and viaduct have the significant difference in the radio wave propagation characteristics. In 3GPP TS36.104, only LOS path is taken into account in HSR open space and tunnel scenarios. However, the existing measurement results [35, 174] have stated that MPCs also exist in typical HSR environment. These MPCs with different delays result in the frequency selective fading to channel responses, which is important for future wideband communication system design [175]. In this section, the channel dispersion in the composite scenario that is composed of cuttings and viaduct are investigated.

\subsubsection{Measurement Scenarios and Configurations}

The wideband measurements have been conducted in a composite scenario, which is located in the "Datong-Xi'an" HSR line (Xinzhou, Shanxi Province, China). As illustrated in Fig. 5.4, the entrance of the composite scenario is a mountain tunnel of $9.2 \mathrm{~m}$ high. Then, two cuttings regions with $130 \mathrm{~m}$ and $100 \mathrm{~m}$ long are connected by a viaduct of $250 \mathrm{~m}$ long. The multipath components reflected or scattered by the sidewalls can lead to severe fading. Moreover, a semi-enclosed space is generated by the cutting structure combining with the mountain, which can accommodate a large sum of MPCs. The propagation mechanism in this composite scenario provides a good reference for HSR communication system design.

For evaluating the effects of MPCs on the propagation in composite scenario, a series of wideband measurements have been carried out at $950 \mathrm{MHz}$ and 2150 $\mathrm{MHz}$. The channel sounder transmitter mounted with the transmitting antenna 

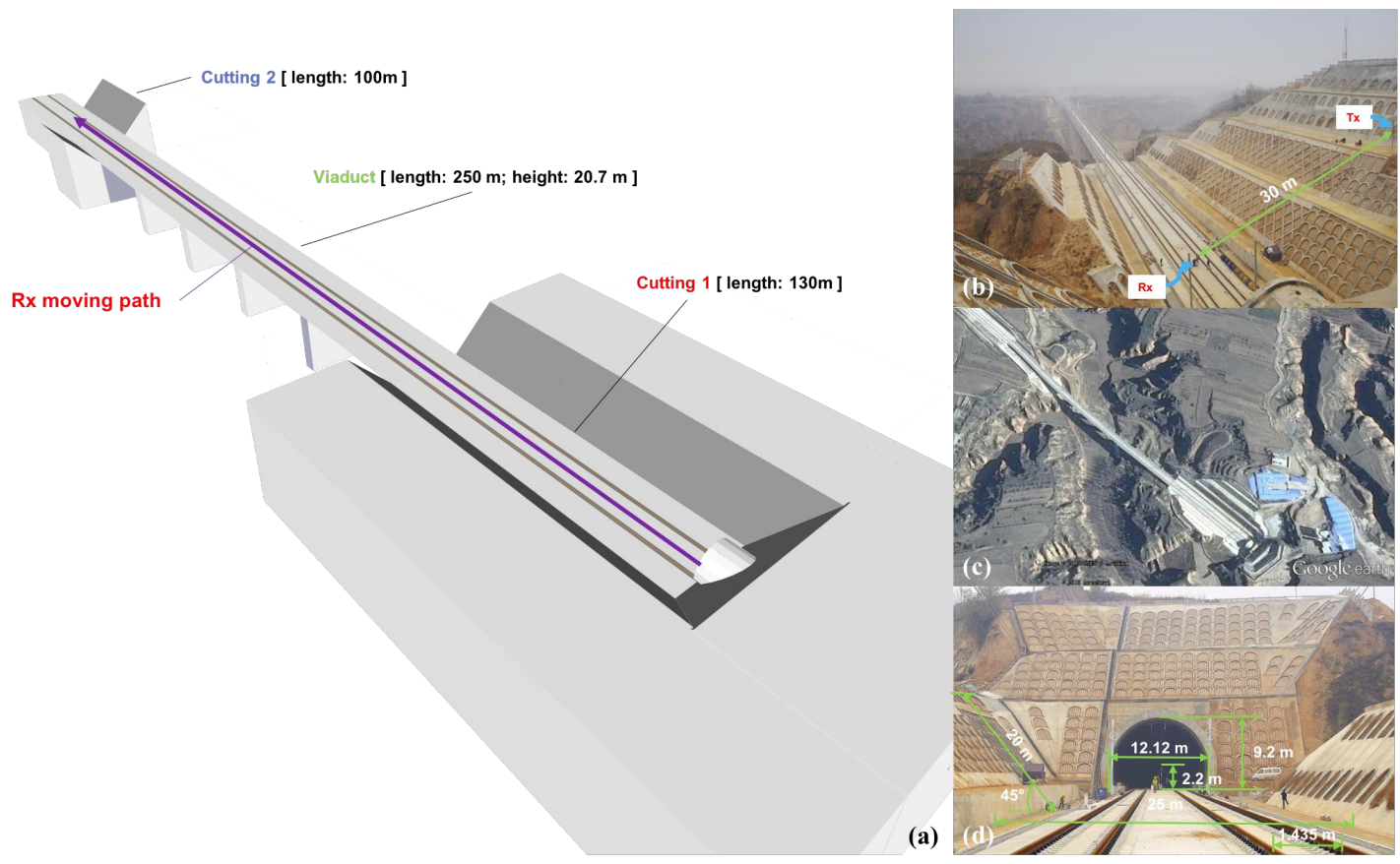

Figure 5.4. The measurements in the HSR composite scenario: (a) sketch of the composite scenario; (b) photo of the test site; (c) aerial view of the HSR composite scenario from google earth; (d) photo of the tunnel portal.

are placed on a platform of the right steep wall. The height of transmitting system is close to the left steep wall and near to the mountain tunnel portal. The downward inclination angle of transmitting antenna is $5^{\circ}$. The channel sounder receiver attached with receiving antenna are located on the rail track and 30 meters distant from the transmitting system as shown in Fig. 5.4 (b). During the measurements, the receiving system is moved along the track in a straight line, and recording the average PDPs at 50 different positions in the composite scenario.

As shown in Fig. 5.4 (c), the measurement environment can be divided into four regions based on the geometric information and expected density of MPCs. Region I: the first few locations near to the tunnel portal, where sounding by the cutting steep walls and mountain thus forming a restricted space full of MPCs; Region II (cutting 1): the first cutting region with two steep walls, which can reflect the transmitting waves and create some MPCs; Region III: the space occupied by the viaduct, where MPCs are quite a few in this open area. Region 
IV (cutting 2): the second cutting area that few MPCs could exist in these locations. The configuration is optimal for typical HSR environments where principal scattering objects are located near to the track. The test sample interval is 50 ns and approximately 20000 channel impulse response (CIR) for each snapshot are collected. Each two neighboring snapshots are separated nearly $10 \mathrm{~m}$. The distance between $\mathrm{Tx}$ and $\mathrm{Rx}$ varies from $30 \mathrm{~m}$ to $458 \mathrm{~m}$.

\subsubsection{Measurement Results Analysis}

The wireless channel fading characterization is essential for the physical layer design of communication system. It is helpful for designing fading countermeasures including transmission/reception diversity, error correction coding. Thus, it is of great significance to parameterize the fading properties of HSR channels in various scenarios.

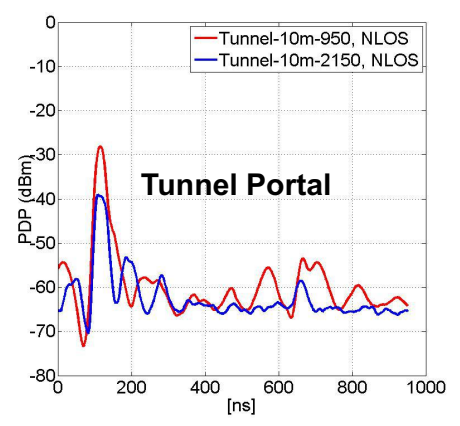

(a)

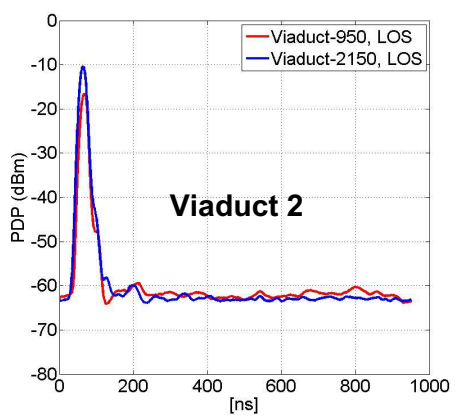

(d)

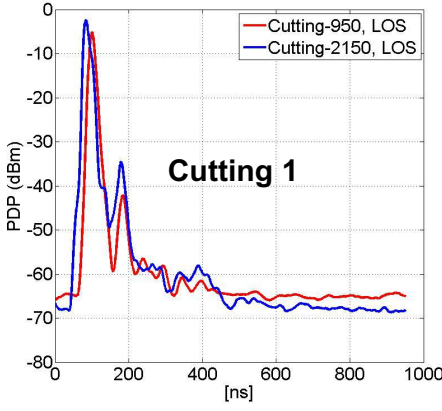

(b)

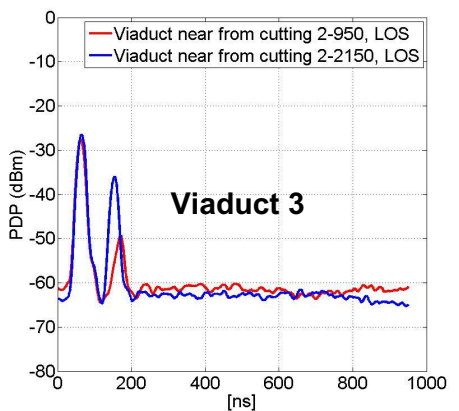

(e)

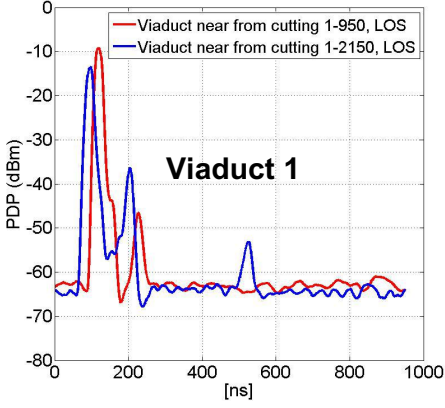

(c)

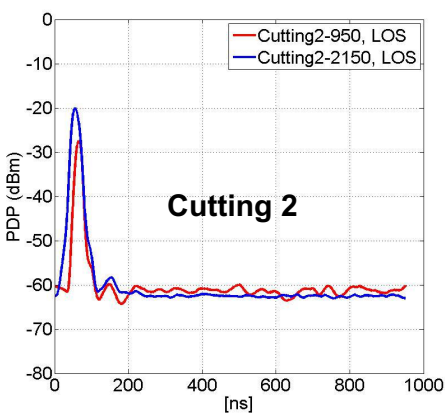

(f)

Figure 5.5. Representative PDPs in different regions:(a) tunnel portal; (b) cutting 1; (c) the part of viaduct close to cutting 1 (Viaduct 1); (d) middle part of viaduct (Viaduct 2); (e) the part of viaduct close to cutting 2 (Viaduct 3); (f) cutting 2. 
The railway base station is usually installed on the side of the rail track. Therefore railway environment is a typical LOS propagation scenario except for the tunnel part. In general, the wave coming from direct path contributes the dominant component of the received signal. Besides, the waves coming from other directions that are generated by multipath components are scattered or reflected by the steep walls or hills, which causes the time delay spread.

The representative PDPs in different regions as examples are demonstrated in Fig. 5.5. Upon the Rx being in Region I, i. e., near the tunnel portal, the direct path from the transmitter to the receiver is blocked by the tunnel roof in the shadowed area. Therefore, an NLOS situation is presented in Region I. PDPs at both frequencies show weak dominant component and long time delay in Fig. 5.5 (a). Especially, the delay spread at $950 \mathrm{MHz}$ is longer and more intense multipath effect occurs than at $2150 \mathrm{MHz}$. Upon the Rx being in Region II, i. e., within the cutting 1 region, there are strong LOS components and follow few delay spread caused by the scattering from the cutting steep walls at both frequencies, as shown in Fig. 5.5 (b). When Rx reaches at the first part of Region III, i. e., the first part of the viaduct near cutting 1 , the LOS component is attenuated, whereas the impact of cutting 1 is still presented. Thus the PDPs in Fig. 5.5 (c) have similar MPCs with slight weaker dominant LOS components. Moreover, some distant scatters may contribute some weak multipath components. In Fig. 5.5 (d), the Rx arrives at the middle part of the viaduct, where MPCs are hard to be detected because of the disappearance of reflection surfaces, therefore the delay spread is greatly decreased. Once the Rx approaching cutting 2, the LOS components are further declined as the separations between $\mathrm{Tx}$ and $\mathrm{Rx}$ are increased. In the meantime, some reflections from cutting 2 are received, which leads to some certain delay spread as shown in Fig. 5.5 (e). Apparently, there is only one dominant path can be found in Fig. 5.5 (f). Since the size of cutting 2 is much smaller and asymmetric with lower height (see Fig. 5.4), the time delay caused by the MPCs is very short and close to the LOS component. Therefore, the remote MPCs originated by the cutting 2 are relatively insignificant to be resolved on the PDPs in Region IV, which results in an addition effect on the dominant component.

Furthermore, the PDP fluctuations along the track at $950 \mathrm{MHz}$ and 2150 


\section{Propagation in High Mobility Scenarios}

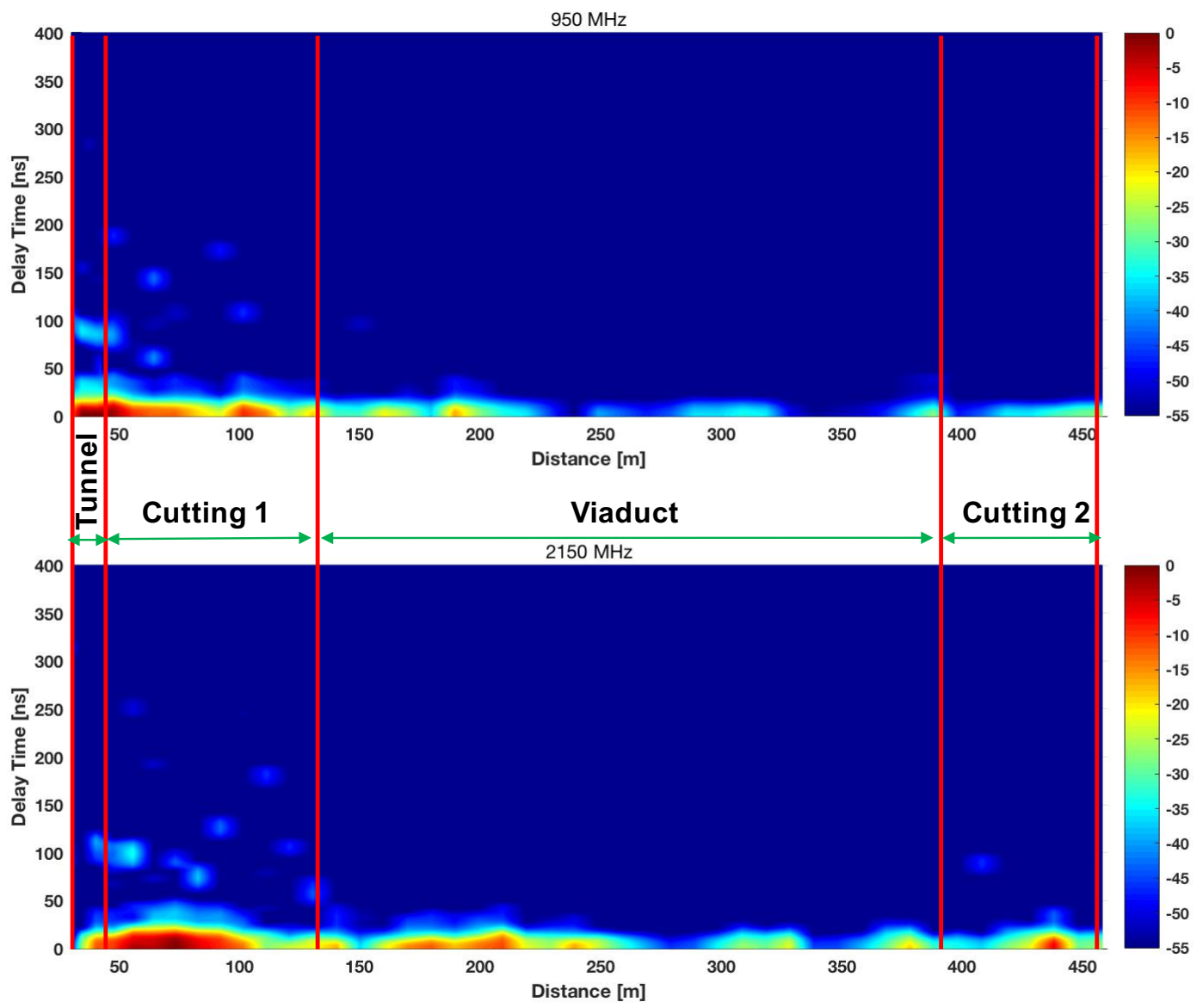

Figure 5.6. PDPs along the cutting and viaduct in the composite region at 950 $\mathrm{MHz}$ and $2150 \mathrm{MHz}$

$\mathrm{MHz}$ are shown in Fig. 5.6. Generally, propagations at two frequencies present quite similar behaviors with the change in distance. In the first $150 \mathrm{~m}$, the propagations at both $950 \mathrm{MHz}$ and $2150 \mathrm{MHz}$ perform quite a clear delay spread. More obvious delay spread appears when the receiver is closer to the mountain tunnel portal, which is due to the strong reflections from the wall above the tunnel and steep walls. Then, the delay spread presents a trend of shortening when the receiver moves along the cutting, which can be explained by the more open space. However, there are still rich multipath components in the first cutting. When the separation between receiver and transmitter reaches to $150 \mathrm{~m}$, i.e., the viaduct entrance on the open area, the delay spread is sharply decreased. Thus, a large number of MPCs die away, and the propagation is dominated by the direct ray. 
Once the receiver is approaching the remote cutting, reflections increases and results in delay spread rising slightly. The region boundaries are also marked on Fig. 5.6 to clarify the differences among the regions.

\subsubsection{Time Dispersion Analysis}

As discussed in Section 2.2.3, multipath propagation leads to channel dispersion in the time domain. To analyze the delay spread profile on the basis of wideband measurement, a common way is grossly quantifying the channel by excess delay and RMS delay spread in a specific scenario. These parameters describe the time dispersive nature of the channel and can be applied to calculated the number of channel taps. Then a region-based TDL model can be established for different areas in the composite scenario. The PDPs are normalized by the first detectable peak path [189]. The excess delay is the maximum delay above a certain threshold above the noise floor, which reveals the maximum relative distance between the receiver and reflection/scattering surfaces. The commonly quantified RMS delay spread is the square root of the second central moment of the PDP and is given by Eq. 4.6, which can be used to extract the number of channel taps.

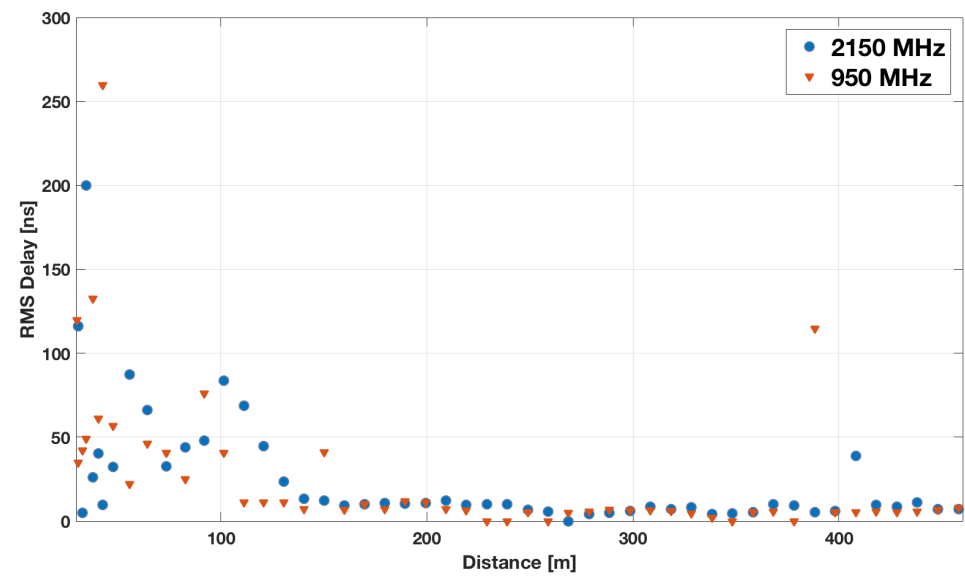

Figure 5.7. RMS delay spread at two frequencies

The average excess delay, average RMS delay spread and the average number of channel taps in each region are summarized in Table. 5.4. The RMS delay along the measurement points at $950 \mathrm{MHz}$ and $2150 \mathrm{MHz}$ are presented in Fig. 
5.7. The change of RMS delay spread along the Rx's moving path is interpreted as the evidence of above discussions. The resolved MPCs in each area of the composite scenario are different, and the general propagation mechanism can be explained with the geometric knowledge in each region. Specifically, as the $\mathrm{Rx}$ moving from a restricted space to an open area, the average RMS delay decreases from about 70-100 ns to 5-7 ns. Correspondingly, the number of channel taps varies with the same downward. Note, even though the average excess delay in Region IV is smaller than Region III, the RMS delay values are just the opposite. It means few MPCs with a short delay may exist in cutting 2 due to the steep walls as discussed above, which imply an potential extra resolvable channel tap in this region.

Table 5.4. Statistical results for modeling the time dispersion at two frequencies

\begin{tabular}{c|c|c|c|c|c}
\hline \hline \multicolumn{2}{c|}{ Region Index } & I & II & III & IV \\
\hline \multicolumn{2}{c|}{ Corresponding Scenario } & Tunnel & Cutting 1 & Viaduct & Cutting 2 \\
\hline \multirow{2}{*}{ Avg. Excess Delay } & $950 \mathrm{MHz}$ & $270.1 \mathrm{~ns}$ & $105.6 \mathrm{~ns}$ & $36.8 \mathrm{~ns}$ & $21.7 \mathrm{~ns}$ \\
& $2150 \mathrm{MHz}$ & $165.3 \mathrm{~ns}$ & $129.6 \mathrm{~ns}$ & $31.2 \mathrm{~ns}$ & $31.3 \mathrm{~ns}$ \\
\hline \multirow{2}{*}{ Avg. RMS Delay Spread } & $950 \mathrm{MHz}$ & $106.4 \mathrm{~ns}$ & $32.6 \mathrm{~ns}$ & $5.3 \mathrm{~ns}$ & $16.2 \mathrm{~ns}$ \\
& $2150 \mathrm{MHz}$ & $71.3 \mathrm{~ns}$ & $43.3 \mathrm{~ns}$ & $7.5 \mathrm{~ns}$ & $13.7 \mathrm{~ns}$ \\
\hline \multirow{2}{*}{ Number of Channel Taps } & $950 \mathrm{MHz}$ & $4 \sim 5$ & 2 & 1 & $1 \sim 2$ \\
& $2150 \mathrm{MHz}$ & $3 \sim 4$ & 2 & 1 & $1 \sim 2$ \\
\hline \hline
\end{tabular}

\subsubsection{D Ray Tracing}

The radio propagation environment properties greatly influence the wireless communication systems[186], particularly for a deterministic model. Since the radio waves in different scenarios have different propagation mechanisms, precisely 3D reconstruction of the propagation environment is a necessary prerequisite for deterministic modeling approach. On the basis of the re-produced environment, the radio channel behavior of the composite scenario as mentioned above can be simulated by the deterministic propagation model. To begin with, the dimensions and altitudes of the viaduct, cutting and barriers have been measured by a laser 
range finder. Then the GPS information and landscape of the test site have been obtained by satellite image from Google Earth. Consequently, a complete vector database for 3D environment reconstruction can be established by gathering all the information above.

For the 3D reconstruction, the major objects in the realistic HSR can be can be classified as the large-scale structure and the small-scale structure. The large-scale structural components include ballestless track, cutting, tunnel, and viaduct are set as triangles and polygons with different electromagnetic parameters. The pylons and vegetation as the small-scale structures are defined to finitelength conductor cylinders and an 8-facet polygonal prism. The main propagation mechanism is set to the scattering rather than reflection. Eventually, the real test scenario is reconstructed by the composite structure of the tunnel, cutting, and viaduct as shown in Fig. 5.8. Also, the electromagnetic parameters of all objects, the positions of transmitter and receiver, and the directions of antennas are included in the scenario model.

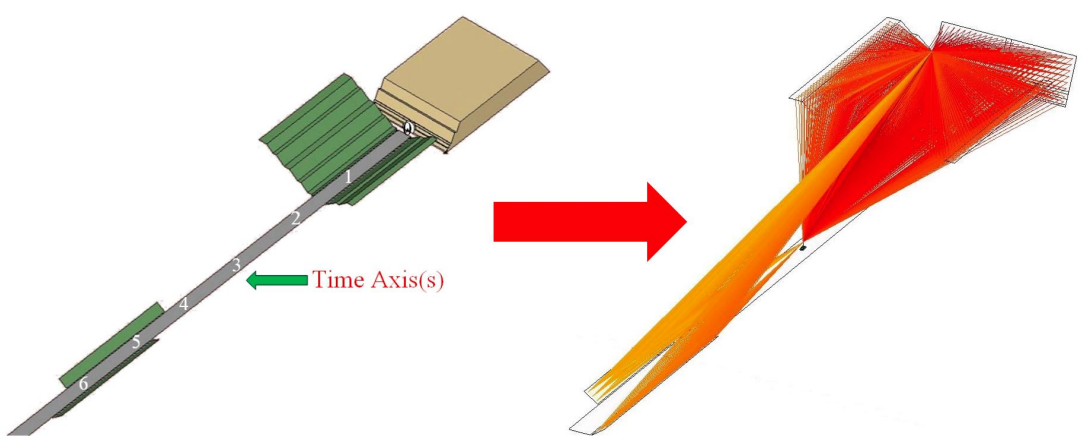

Figure 5.8. Scenario model of the HSR composite scenario and a snapshot of ray tracing process.

As the snapshot of the ray-tracing process in Fig. 5.8 shows, the 3D rayoptical based channel simulation can be used to establish 3D deterministic channel model. The information of each ray is contained in the deterministic model, such as path type, path delay, path loss, and the 3D angular properties. Therefore, the fine details of the propagation channel are provided, for instance, the ray type, its angle of arrival and departure, traveled distance, and equivalent complex baseband representation. 


\section{Stochastic Description}

As described in Chapter 2, the radio propagation in linear time-varying (LTV) channel is mainly suffered multipath propagation and the Doppler effect. Based on the discussion in [190], assuming a LTV channel with continuum scatterers, the received signal is generally given by:

$$
r(t)=\int_{-\infty}^{+\infty} \int_{-\infty}^{+\infty} S_{\mathbf{H}}\left(\tau, f_{d}\right) s(t-\tau) \exp \left(j 2 \pi f_{d} t\right) d \tau d f_{d}
$$

where $S_{\mathbf{H}}\left(\tau, f_{d}\right)$ denotes the delay-Doppler spreading function of the LTV channel $\mathbf{H}$. $S_{\mathbf{H}}\left(\tau, f_{d}\right)$ presents the overall complex attenuation and scatterer reflectivity of all paths with the time delay $\tau$ and Doppler frequency $f_{d}$ (see Eq. 2.25), which characterizes the channel dispersion in both time and frequency domains. Particularly, if $P$ discrete ideal point scatterers are simulated, the Eq. 5.1 can be rewritten as follows:

$$
S_{\mathbf{H}}\left(\tau, f_{d}\right)=\sum_{p=1}^{P} h_{p} \delta\left(\tau-\tau_{p}\right) \delta\left(f_{d}-f_{d, p}\right)
$$

where $h_{p}, \tau_{p}$ and $f_{d, p}$ denote the complex attenuation factor, time delay, and Doppler frequency at $p^{t h}$ path. The time-varying impulse response $h(t, \tau)$ is a Fourier transform of spreading function $S_{\mathbf{H}}\left(\tau, f_{d}\right)$.

A wireless channel can be treated as wide-sense stationary (WSS) if there are no great variations in a stationarity time interval [191, 192]. Thus, WSS channels are stationary in time with the uncorrelated Doppler frequency coefficients. Besides, the uncorrelated scattering (US) channels are featured with uncorrelated time delay coefficients and stationary in frequency. Therefore, for the joint hypothesis of wide-sense stationary uncorrelated scattering (WSSUS) channel, the spreading function $S_{\mathbf{H}}\left(\tau, f_{d}\right)$ is a $2 \mathrm{D}$ white process, and thereby offering a secondorder statistical characterization of the (time-frequency) TF domain [190],

$$
E\left\{S_{\mathbf{H}}\left(\tau, f_{d}\right) S_{\mathbf{H}}{ }^{*}\left(\tau^{\prime}, f_{d}^{\prime}\right)\right\}=C_{\mathbf{H}}\left(\tau, f_{d}\right) \delta\left(\tau-\tau^{\prime}\right) \delta\left(f_{d}-f_{d}^{\prime}\right)
$$

where $S_{\mathbf{H}}^{*}\left(\tau^{\prime}, f_{d}^{\prime}\right)$ is the complex conjugation of $S_{\mathbf{H}}\left(\tau^{\prime}, f_{d}^{\prime}\right) . C_{\mathbf{H}}\left(\tau, f_{d}\right)$ is the 
scattering function of channel $\mathbf{H}$, which characterizes the average strength of scatterers with delay $\tau$ and Doppler frequency $f_{d}$. Hence, $C_{\mathbf{H}}\left(\tau, f_{d}\right)$ statistically describes the TF dispersion in a WSSUS channel.

Furthermore, one of the marginals of the scattering function is sufficient for time/frequency domain analysis in some cases. Specifically, the power delay profile and power Doppler profile are defined by $c_{\mathbf{H}}^{(1)}(\tau)$ and $c_{\mathbf{H}}^{(2)}\left(f_{d}\right)$, respectively, as

$$
c_{\mathbf{H}}{ }^{(1)}(\tau) \triangleq \int_{-\infty}^{+\infty} C_{\mathbf{H}}\left(\tau, f_{d}\right) d f_{d}, \quad c_{\mathbf{H}}{ }^{(2)}\left(f_{d}\right) \triangleq \int_{-\infty}^{+\infty} C_{\mathbf{H}}\left(\tau, f_{d}\right) d \tau .
$$

These two profiles are used to analyze mean excess delay, mean Doppler shift, RMS delay spread, and RMS Doppler spread. The mean excess delay and mean Doppler shift are defined by the the first moments and calculated as follows,

$$
\bar{\tau} \triangleq \frac{1}{\rho_{\mathbf{H}^{2}}} \int_{-\infty}^{+\infty} \tau c_{\mathbf{H}}{ }^{(1)}(\tau) d \tau, \quad \bar{f}_{d} \triangleq \frac{1}{\rho_{\mathbf{H}^{2}}} \int_{-\infty}^{+\infty} f_{d} c_{\mathbf{H}}{ }^{(2)}\left(f_{d}\right) d f_{d}
$$

where $\rho_{\mathbf{H}}^{2}$ is the average power attenuation.

Moreover, RMS delay spread and RMS Doppler spread are the square root of the second central moments of $c_{\mathbf{H}}^{(1)}(\tau)$ and $c_{\mathbf{H}}^{(2)}\left(f_{d}\right)$, which are given by:

$$
\sigma_{\tau} \triangleq \frac{1}{\rho_{\mathbf{H}}} \sqrt{\int_{-\infty}^{+\infty}(\tau-\bar{\tau})^{2} c_{\mathbf{H}}{ }^{(1)}(\tau) d \tau}, \quad \sigma_{f_{d}} \triangleq \frac{1}{\rho_{\mathbf{H}}} \sqrt{\int_{-\infty}^{+\infty}\left(f_{d}-\bar{f}_{d}\right)^{2} c_{\mathbf{H}}{ }^{(2)}\left(f_{d}\right) d f_{d}}
$$

These globe channel parameters provide key properties of LTV channel in TF domain, which are relevant for wireless communication channel design.

\section{Validation}

It has been widely known that the main disadvantage of ray tracing approach is time-consuming. Therefore, in order to improve the efficiency of RT algorithm, the objects involved in the scenario reconstruction model, such as the external wall of the tunnel, viaducts, and cuttings are predominant for the HSR environment. 
In other words, the objects that are invisible, remote, and lower than the track in a real environment are excluded in the simulation. Configuring the ray tracer in this way speeds up the processing of computation on each snapshot, then significant time savings could be realized entirely (up to 70\%).

Defining $\lambda / 16$ as the sampling interval in the RT simulation, where $\lambda$ is the wavelength of the center frequency. The speed of the train is set to $300 \mathrm{~km} / \mathrm{h}$. As a result, the total simulation duration is about $6.4 \mathrm{~s}$. The remained parameters for RT simulation configurations remain consistent with the measurement settings. The time axis is marked on the import scenario model and indicates the train position, as illustrated in Fig. 5.8.
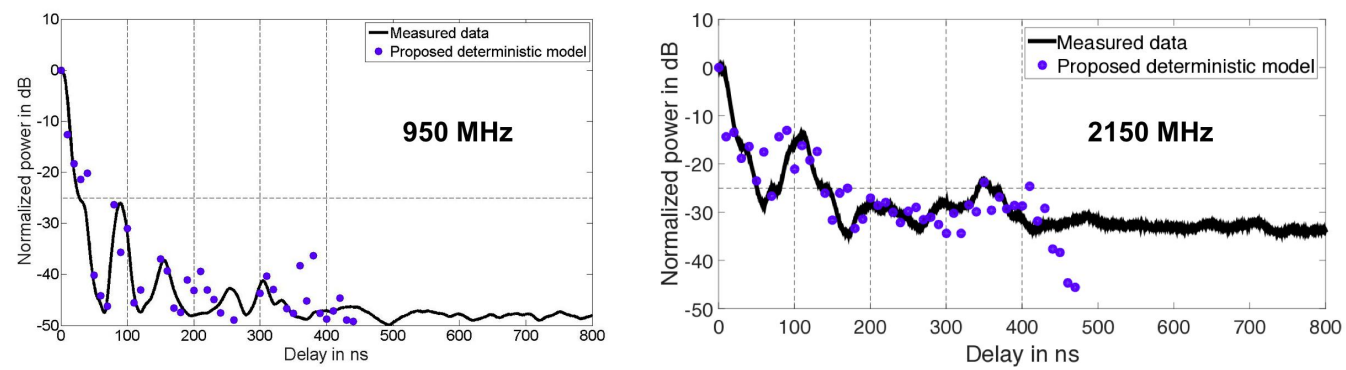

Figure 5.9. Comparisons of the PDPs between the measurement and the proposed deterministic model at two frequencies

Fig. 5.9 shows the comparison of PDPs from the measurement and the prediction of RT simulation in one snapshot at both $950 \mathrm{MHz}$ and $2150 \mathrm{MHz}$. The measured and predicted results have a good agreement both on the normalized power and multipath delay structure. Due to the simply modeling of remote scatterers, there are some delay points longer than 300 ns are not precisely matched. However, the mismatch of these points is unimportant since their normalized power are relatively low. Fig. 5.10 gives the PDPs along the scenario time points at two frequencies. The variation trends of the statistical properties are basically consistent with the measurement results. The delay obtained by deterministic channel model is prevalently shorter than the measured delay, whereas the power of MPCs is higher than the measurement results owing to the scenario simplification. Nevertheless, the deterministic channel model is generally applicative for further small-scale fading and Doppler characteristic analysis. 
5. Propagation in High Mobility Scenarios
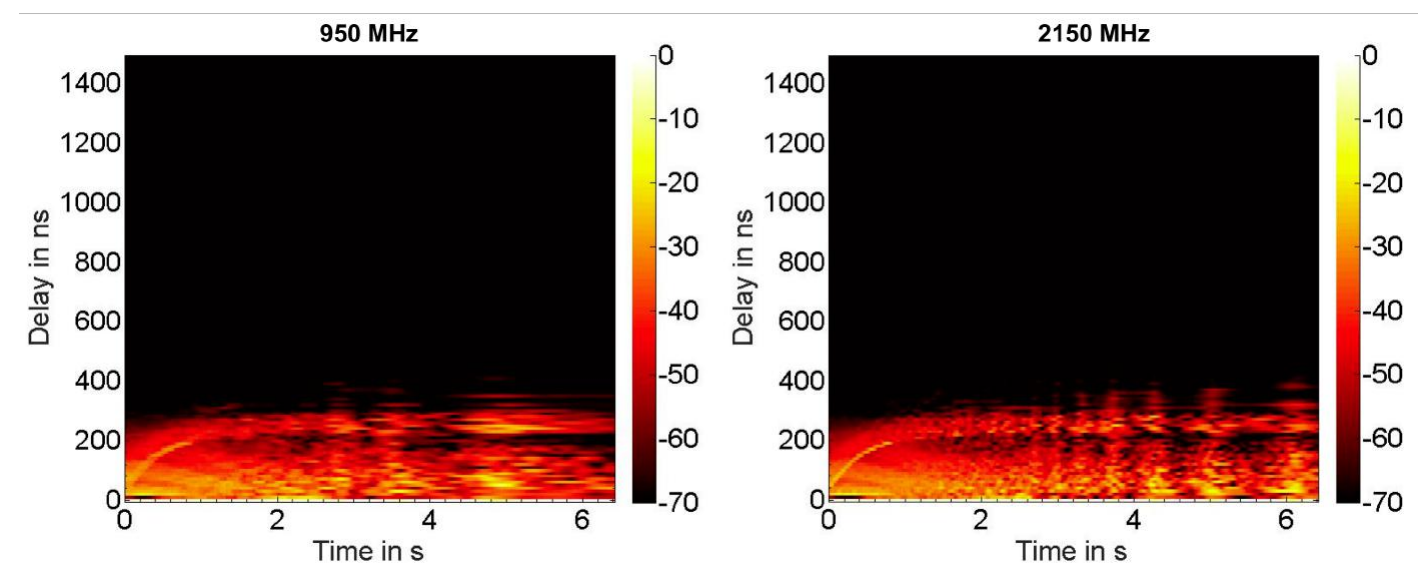

Figure 5.10. PDP along scenario time at different frequencies

\section{Small-scale fading and Doppler statistical properties analysis}

Since the transmitter bandwidth, output power, antenna parameters and position in RT simulation is consistent with the field measurement, the small scale fading can be extracted and analyzed based on the RT simulation. After removing the large-scale fading effect by averaging received power in each sliding window (window size: $40 \lambda$ ), the small-scale fading as the function of time in the composite scenario is obtained, as shown in Fig. 5.11. The significant differences are observed as the small-scale fading characteristics in a different location at both $950 \mathrm{MHz}$ and $2150 \mathrm{MHz}$. The fading characteristics are investigated by employing the fading depth (FD) and maximum fading depth (Max. FD). The FD is the signal level difference between the $1 \%$ and $50 \%$, and Max. FD is the corresponding difference between $50 \%$ and maximum fading value. The fading characteristics at two frequencies in each time frame are given in Table 5.5. The different zones are partitioned by the scenario time axis in Fig. 5.8. It can be observed that the higher fading values in zone 1 and zone than most of the rest zones, thus the rapid changes in signal strength can be predicted in the restricted area with serious multipath effects. Then, FD in zone 2 is bigger than in zone 3 for both $950 \mathrm{MHz}$ and $2150 \mathrm{MHz}$, even though these two zones are both located in viaduct region. This is because zone 2 is closer to cutting area than zone 3 . Besides, a prominent Max. FD about $-59 \mathrm{~dB}$ is presented in zone 4 at $2150 \mathrm{MHz}$, 
which is much deeper than the rest zones at $2150 \mathrm{MHz}$ and all the cases at 950 $\mathrm{MHz}$.
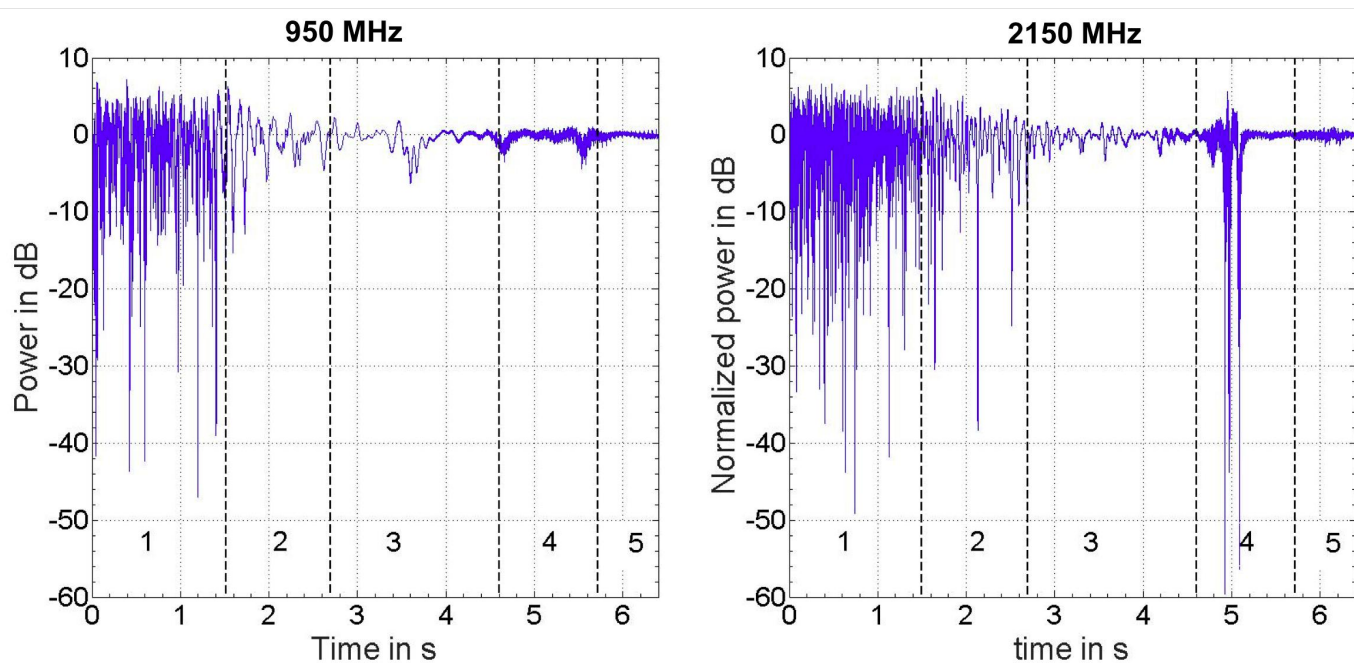

Figure 5.11. Small-scale fading along the scenario time at two frequencies.

Table 5.5. Analysis of normalized short-term fading

\begin{tabular}{c|c|c|c|c|c}
\hline \hline Zone index & 1 & 2 & 3 & 4 & 5 \\
\hline Time frame $[\mathrm{s}]$ & $0-1.5$ & $1.5-2.7$ & $2.7-4.7$ & $4.7-5.6$ & $5.6-6.4$ \\
\hline FD at $950 \mathrm{MHz}[\mathrm{dB}]$ & 6.1 & 5.2 & 2.1 & 0.7 & 0.9 \\
\hline Max.FD at $950 \mathrm{MHz}[\mathrm{dB}]$ & 46.07 & 14.87 & 6.02 & 1.68 & 4.38 \\
\hline FD at $2150 \mathrm{MHz}[\mathrm{dB}]$ & 15.4 & 17.9 & 2.8 & 15.5 & 0.7 \\
\hline Max.FD at $2150 \mathrm{MHz}[\mathrm{dB}]$ & 48.37 & 37.87 & 3.35 & 59.04 & 1.37 \\
\hline \hline
\end{tabular}

As the receiver (simulated high-speed train) passing by the transmitter from the tunnel, the mean Doppler shift rapidly decreases from $200 \mathrm{~Hz}$ to $-600 \mathrm{~Hz}$ at $2150 \mathrm{MHz}$ and from $100 \mathrm{~Hz}$ to $-260 \mathrm{~Hz}$ at $950 \mathrm{MHz}$, as shown in Fig. 5.12. Moreover, the mean Doppler shift and RMS Doppler spread at two frequencies are presented in Fig. 5.13. By means of comparisons between Fig. 5.13 and Fig. 5.11, TF domain analysis can be concluded. The trend of fading depths is consistent with the RMS Doppler spreads, where the test point with larger fading depth also has a lager RMS Doppler spread. A possible reason is the dense scattering 
components from asymmetric cutting creating severe Doppler spread. Therefore several Doppler-shifted waves are superposed. Furthermore, a deep fading is obtained at the test point that has a long delay spread. Generally, Doppler spread at $950 \mathrm{MHz}$ is not as large as at $2150 \mathrm{MHz}$ by the mean of simulation, which means no serious fading with larger FD has occurred. Note that the angle-modulated radio signals can be impaired by the phase shifts caused by the Doppler effect at the receiver side. Hence, directional receiving antenna or diversity technique has to be considered in the wireless communication system design and development to mitigate the Doppler effects.
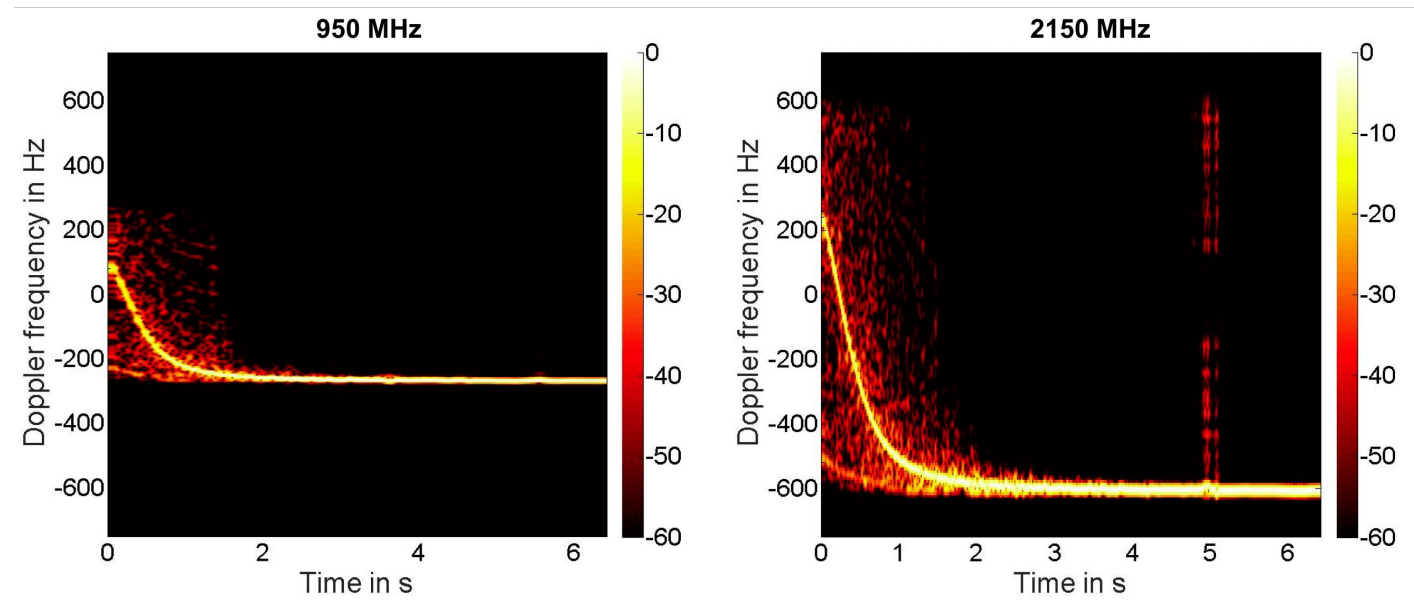

Figure 5.12. Doppler power spectrum along scenario time at two frequencies.

\subsection{Discussion}

In this chapter, the key features and challenges for channel measurement and modeling in high mobility scenarios are introduced. Then a set of wideband propagation measurements in real HSR station and composite scenario at 950 $\mathrm{MHz}$ and $2150 \mathrm{MHz}$ are presented and analyzed as the practical test cases. The customized channel sounder specially designed for railway environment has been employed and achieved good performances. On the basis of the measurement results, the key parameters for analyzing the time dispersion properties in HSR scenarios are extracted. These parameters including the excess delay, the RMS 

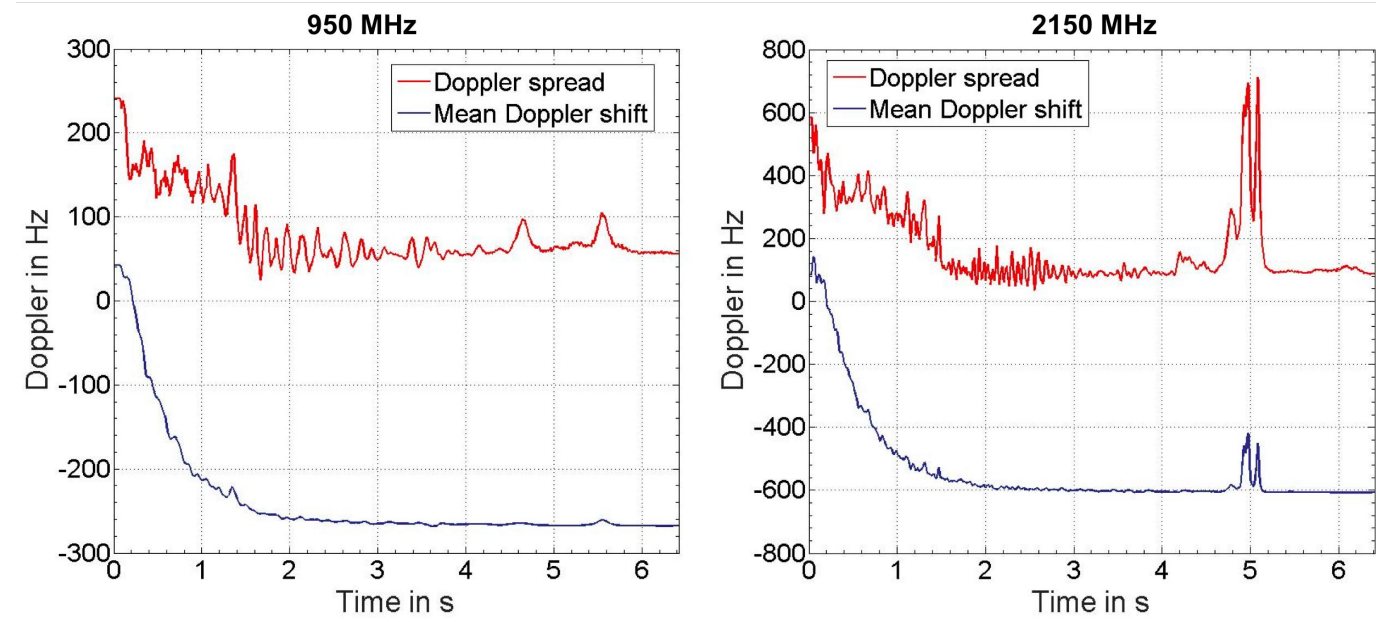

Figure 5.13. Mean Doppler shift and RMS Doppler spread along scenario time at two frequencies.

delay spread, and the number of channel taps, are calculated and compared to different configurations and situations. Furthermore, the TDL channel models can be established in detail in different regions. For instance, in the HSR station, the LOS and NLOS have different propagation mechanisms that result in different time delay characterizations. As the second test scenario, the HSR composite scenario includes some typical HSR scenarios: tunnel, cutting, and viaduct. The channel measurement and modeling in this complex joint environment are highly interesting and different with the isolated scenario. The measurement results show some important appearances in these composite regions channel: the relatively small difference of time delay at two frequencies; the more MPCs in the cutting and tunnel portal result greater RMS delay than the rest regions; time dispersion characterization in viaduct is influenced by the two connected cuttings, i.e., the middle part of the viaduct has shown less time delay than the two ends. Moreover, the number of channel taps varies in different regions with the same trend of RMS delay. For obtaining more details of the radio channel in time and frequency domains, a deterministic channel model of the composite regions are proposed based on 3D ray tracing. The real measurement site is simply reconstructed in $3 \mathrm{D}$ to conduct the $3 \mathrm{D}$ ray tracing approach. The simulation results are compared and validated based on the measured data gathered in the same regions. The transition regions are explicitly recognizable by the normalized 
small-scale fading. Also, the Doppler characteristic analysis in frequency domain proves the necessity of studying composite regions can rely on the combination of measurement and simulation. The interferences of the scattering components from cuttings create not only deeper fading but also delay and Doppler dispersion. Since the propagation channels in HSR station and composite regions have a strong impact on the radio coverage, reliability and quality of service of HSR mobile communication networks, the results and models in this chapter can be helpful for the system design, network planning and optimization in HSR complex environments. 


\section{Chapter 6}

\section{Propagation inside Mass Transit Systems}

Mass Transit System (MTS), also widely known as public transport, is a basic mean of providing mobility for the general population in daily life. Buses, trams, subway/railway trains, high-speed trains, including sophisticated driverless vehicles, need an efficient communication system which grows at the same pace as their own operations, all the while as they meet the service availability and safety expectation of their customers [193]. MTS is an essential part of the development of modern society. Therefore, continues control signals, voice and data communication between the BTS and mobile user inside the vehicles, as well as communications between different carriages or vehicles are issues of crucial importance. The wireless communication system for MTS is expected to provide two types of applications [194]:

- Vital Applications: related to signaling and control/command of equipment. For instance, voice communication with the driver, public address, and Intercom systems, etc.; critical data for alarm management, vehicle diagnostics, location, emergencies, etc.; signaling data for driving instructions. Such applications generally require a low bandwidth (several 10 to $100 \mathrm{Kbps}$ ) but a very high degree of availability (at least 99.99\%), robustness and liability (typically a packet error rate of $10^{-3}$ for an approximately 200 byte packet length). Such performance indicators have equally to be 
6. Propagation Inside Mass Transit Systems

fulfilled under high-speed mobility (handover timing when a mobile terminal must change its connection from one fixed base station to another). These vital applications are typically CBTC in the case of metros or ETCS in the case of mainline trains.

- Non-vital Applications: related to non-critical data communications. For example, passenger information; remote maintenance; onboard video surveillance for displaying real-time images from trains; fixed video surveillance for monitoring of tunnels, crossings, and stations; Internet access for advertising and entertainment for passengers, etc. Such non-vital applications generally require much higher bandwidth (several 10s of Mbps in both direction train-to-ground and ground-to-train), together with lesser robustness constraints (a packet error rate of $10^{-2}$ for an approximately 1 MB packet length). The advent of broadband applications such as High Definition (HD) video transmission along with the need to collaborate and network through the Internet, has made the need for these non-vital applications even more relevant.

The challenge for the wireless communication system in coming years is the development of high-capacity $5 \mathrm{G}$ networks. $5 \mathrm{G}$ systems are expected to provide simultaneous broadband services to a large number of users traveling at speeds up to $500 \mathrm{~km} / \mathrm{h}$, the speed achievable by HSR systems, at a data rate of 150 Mbps or higher [165]. Also, rising demand for high speed and high capacity data service on board the MTS stimulates the telecommunication operators and public transportation companies showing a great interest in deploying broadband mobile wireless networks in Mass Transit System (MTS). Nevertheless, providing a high capacity wireless link for the MTS is an interesting difficulty. Typically, an MTS such as the high-speed train with 500 passengers providing 50 Mbps connections to each user would require a connection of several Gbps. This is particularly difficult for MTS moving in a complex environment such as tunnel, cutting, and station. Besides, traveling across the boundaries of areas covered by two different base stations triggers handover. For MTS, hundreds of mobile devices inside MTS trying to handover at the same time results in the group handover and generating the so-called signaling storm. Moreover, a significant 
6. Propagation Inside Mass Transit Systems

shielding effect on the received signal due to the metal construction of the MTS can not be ignored. In the mean time, this extra loss varies in different types of vehicles and environments. To overcome these difficulties, it is necessary to develop moving relay solutions to provide high-quality network inside the MTS, such as the Mobile-Relay technique proposed by 3GPP [195]. Therefore, radio propagation characteristics for wireless communication systems onboard the MTS is essential for future relay system development.

In the last few years, research on communication systems for MTS have mainly focused on the Vehicle-to-Infrastructure (V2I) communications [196], whereas studies on the propagation behavior of wireless links onboard the vehicles are rather little. Propagation properties for different yet related scenarios have been studied. For instance, many research have been conducted in airplane cabins by the mean of simulation [197], narrowband measurements [198, 199, 200], and wideband measurements [201, 202, 203, 204]. Propagation inside small vehicles include bus with passengers [205]; elevator shafts [206] and cars [207, 208] have been characterized. Also, propagation properties in other confined environment such as rooms, corridors [209], and tunnels [210, 211, 212] have been summarized and compared. In these settings, researchers concluded that PLE values vary between 2 and 3 in the airplanes with medium to large size. Whereas the estimated PLE is less than 1 in the cargo bay of a military airplane, which indicates waveguiding effect in small size vehicles [199]. This effect also has been described in [213], [214], and [215] in the case of radio propagation aboard the train in the $2 \mathrm{GHz}$ band. Reflected waves from car-body in a railway tunnel at $2.4 \mathrm{GHz}$ have been estimated by FDTD analysis in [216], which states a nearby train does not significantly affect the radio propagation. Besides, experimental results in [217] have shown an extra $10.4 \mathrm{~dB}$ loss of the signal re-entering through the windows of the train in Inter-Car links at $5 \mathrm{GHz}$. In addition, outdoor-to-indoor high speed channels have been evaluated by means of measurements and simulations in [218] and [219].

In general, wireless network access systems for the railway environment can be classified into five types as shown in Fig. 6.1: BTS-to-Train (B2T) link: the outdoor-to-outdoor link between the BTS and the external antennas of the train; BTS-to-Mobile (B2M) link: the outdoor-to-indoor link between the BTS and 


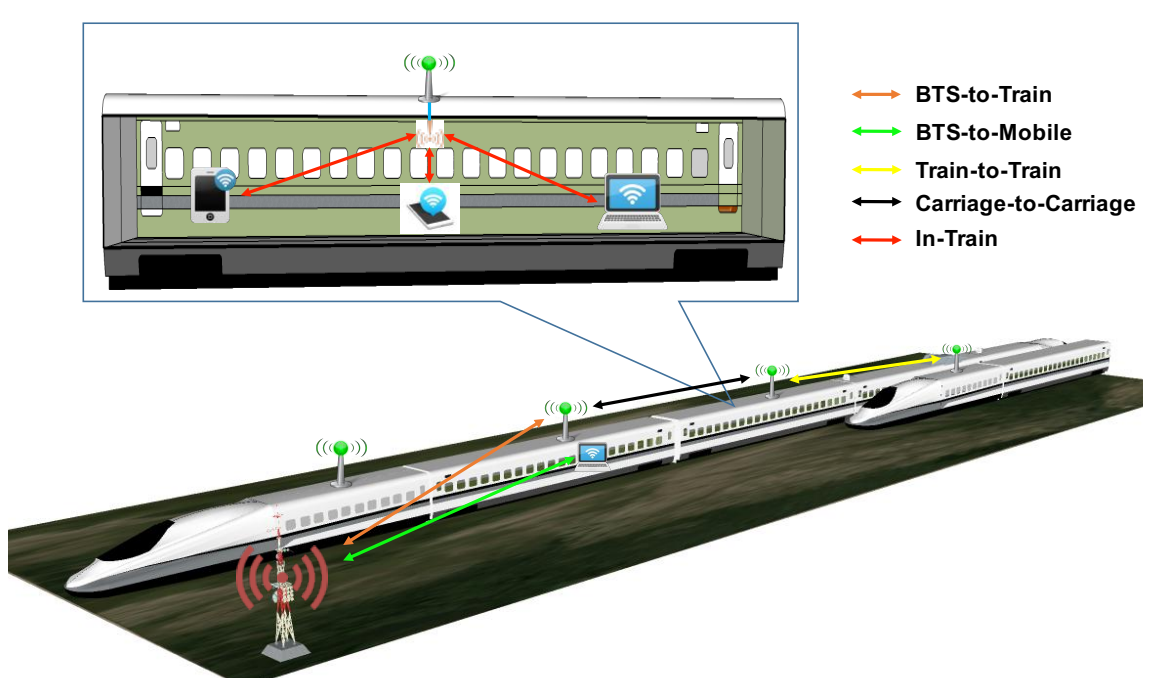

Figure 6.1. Various wireless communication links onboard the train.

a mobile device inside the train. (e.g., a passenger using a mobile device directly attached to the commercial cellular network); In-Train (IN-T) link: the link between a mobile receiving device and a repeater/AP located inside the train. This link can be divided into two separate links: Intra-carriage and Inter-carriage. Depending on the position of the receiver, the communication channel is different in different carriages. Carriage-to-Carriage (C2C)/ Train-to-Train (T2T) link: the communication link between the carriages/ trains along the track.

In the following sections, a series of propagation measurements are presented with aiming to achieve two requirements for future wireless network design in HSR:

1. Considerable radio coverage along the railway track;

2. The efficient signal delivering into the carriage.

For the first layer, the propagation measurement for outdoor-to-indoor HST is described and evaluated based on the test tails with LTE waveforms receiving by the external and internal antennas onboard the train. Then, the propagation measurements inside the train carriages with different configurations are presented and analyzed for layer II. 


\subsection{Layer I: Outdoor-to-Indoor Coverage}

For most of the existing railway communication systems, the normal situation is the mobile devices of passenger receiving the radio signal directly from the distant base station. Whereas the radio signal from the base station has to penetrate the train's body to arrive at the receiver side inside the train. Therefore conventional communication system is hard to provide satisfying network services for the travelers onboard the train. Moreover, continuous radio coverage along the rail tracks is an essential requirement for high-speed train network deployments. The effective coverage aims to avoid the interruption of control signaling and passenger service. However, the extra loss caused by the train's structure is supposed to highly decrease the coverage range of the base station. To quantify the coverage issues for the train and the mobile device inside the train, an empirical study of the LTE base station coverage along the rail track is presented in this section.

\subsubsection{Measurement Scenarios and Configurations}

The outdoor-to-indoor propagation measurement has been conducted at the highspeed rail line between Córdoba and Málaga in Spain, where the commercial HSR operation reaches speeds of up to $330 \mathrm{~km} / \mathrm{h}$. The test track is a segment in the vicinity of Antequera-Santa Ana station (KP 96.800). This track section includes a base station site located at KP $97.075^{1}$ consisting of $2 \mathrm{G} / 3 \mathrm{G}$ commercial equipment mounted on a $40 \mathrm{~m}$ height tower with antennas for various wireless technologies at different frequencies (see Fig. 6.2 (a))

The test MTS in this measurement is the Séneca laboratory train (Talgo A330) provided by the Spanish railway operator $A D I F$, as shown in Figs. 6.2 (b). Séneca is an electric train with $80.92 \mathrm{~m}$ long and holds a maximum operation speed up to $363 \mathrm{~km} / \mathrm{h}$. as shown in Fig. 6.2 (c), the Sneca train provides two sectors of the external antenna on the roof.

For evaluating and comparing the outdoor and indoor communications onboard the train, two links are defined as follows:

- BTS-to-Train (B2T) link with external antennas: The eNodeB (LTE testbed

\footnotetext{
${ }^{1}$ The exact GPS coordinates of the site are $37^{\circ} 4^{\prime} 3.14^{\prime \prime} \mathrm{N}, 4^{\circ} 43^{\prime} 12.52^{\prime \prime} \mathrm{W}$.
} 


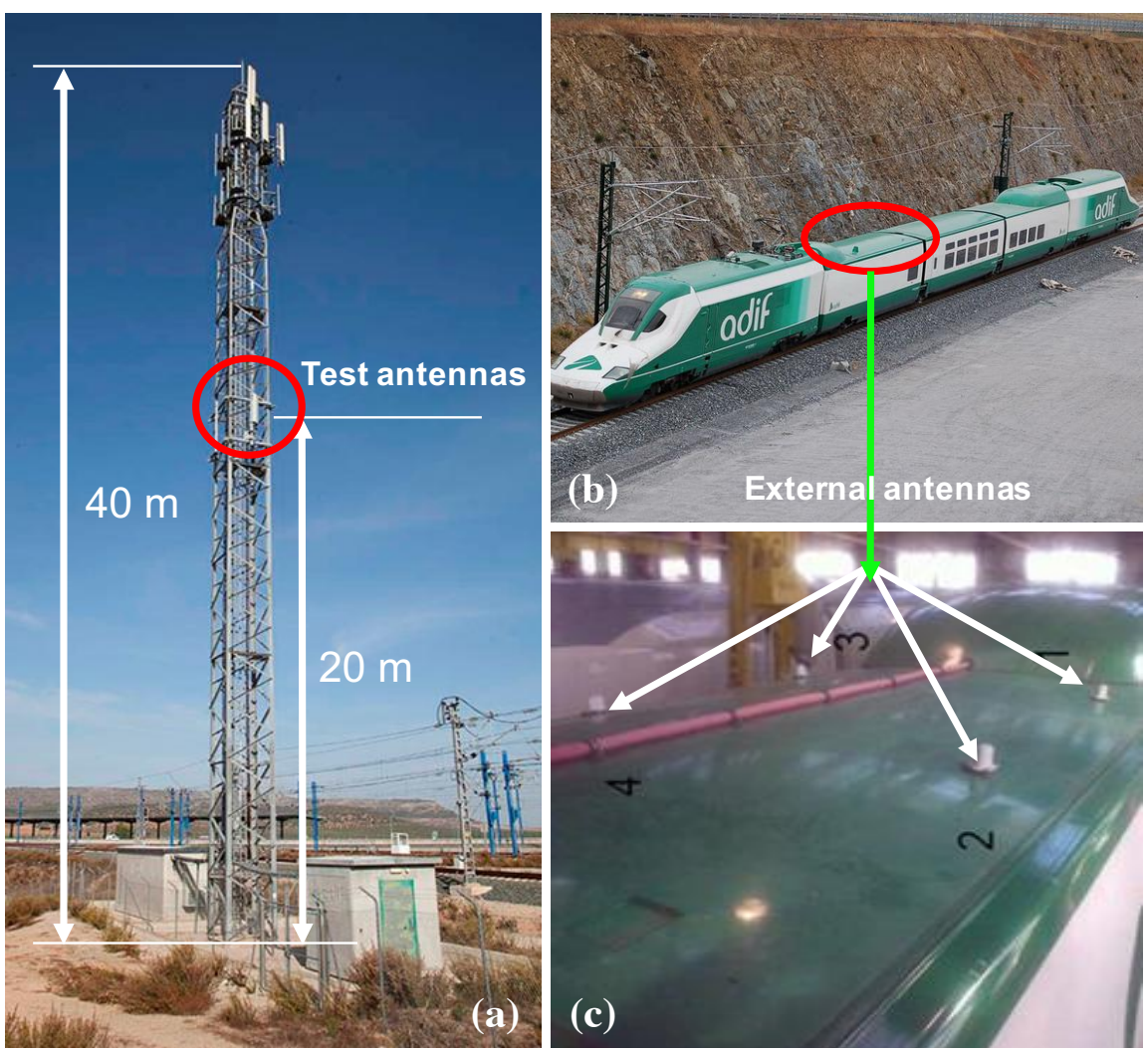

Figure 6.2. Outdoor test environments: (a) LTE BTS tower structure with the eNodeB antennas at $2.6 \mathrm{GHz}$ mounted at a height of $20 \mathrm{~m}$; (b) snapshot of the test train by Pablo Nieto (via: www.ferropedia.es); (c) four external antennas on the roof of the train carriage.

transmitter) is employed to transmit the signal, and firstly received by the outdoor external antennas on the roof of the train.

- BTS-to-Mobile (B2M) link with internal antennas: In the mean time, the LTE testbed receiver mounted inside the train also capture the transmitted signal by the attached indoor antennas.

Moreover, the train moves at a constant speed of $50 \mathrm{~km} / \mathrm{h}$ between KP 93.4 and 102.0, passing in front of the tower where the eNodeB antennas are installed at KP 97.075. The measurement site from Google map is shown in Fig. 6.3 (a). Then, the transmitted LTE signals are simultaneously acquired by the two indoor and the two outdoor antennas. The LTE radio coverage appears in Fig. 6.3 
(b) has been simulated by the 9955 RNP (Radio Network Planning) tool from Alcatel-Lucent. The same configurations such as the antennas orientation, and the parameters have been applied in our measurements: the transmitted signal is constructed by 1024 subcarriers with a $10 \mathrm{MHz}$ bandwidth and $46 \mathrm{dBm}$ transmit power.

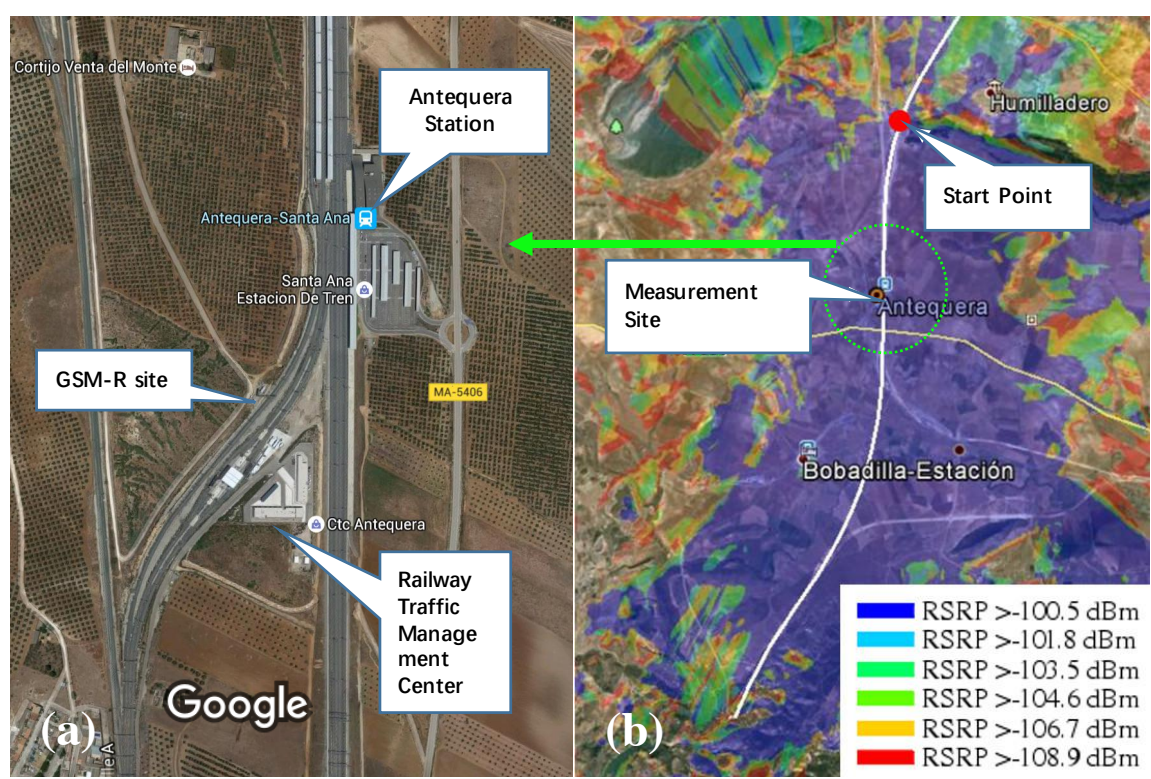

Figure 6.3. Measurement site: (a) measurement site from Google map; (b)BTS with eNodeB $2.6 \mathrm{GHz}$ located at Antequera station. Simulation of the LTE radio coverage along the test track.

\section{LTE Testbed}

A commercial LTE eNodeB with two deployed sectors has been employed in our measurement. The eNodeB has been installed in one of the cabins of the BTS, and two remote radio heads (each one connected to two antenna elements in the same antenna panel) have been mounted on the BTS tower with 20 meters high (see Fig. 6.2 (a)).

Both transmit panel antennas (Moyano MY-DTBSBS17276518) have been oriented with the same directions as the commercial GSM-R antennas already installed in the same tower (see Fig. 6.3 (a)). At the carrier frequency 2.6 GHz, 
these cross-polarized antennas feature a gain of $18 \mathrm{dBi}$ and half-power beamwidth of $62^{\circ}$ (horizontal) and $5^{\circ}$ (vertical). The antenna panel which serves the north sector has an azimuth orientation of $355^{\circ}$ and a tilt of $0^{\circ}$ (both electrical and mechanical), whereas the one radiating for the south sector has an azimuth orientation of $175^{\circ}$ but a total tilt of $-1.4^{\circ}\left(0^{\circ}\right.$ mechanical and $-1.4^{\circ}$ electrical $)$.

The LTE testbed receiver is an evolved version of those described in [220], which consists of two nodes operating in receive-only mode. Each node consists of a single USRP B210 board built from the Analog Devices AD9361 chip. Each node also includes a laptop computer with custom-developed software. More concretely, a multi-thread receiver is adopted in the measurement, which is able to provide the same information in real-time (e.g., captures of the acquired signal spectrum) and store the acquired samples for off-line processing of all the recorded LTE frames.

Both LTE testbed receiver nodes have been installed inside the train. One of them has been connected to two of the four antennas available on the roof of the train carriage, which corresponds to the B2T link. The other receiver has been connected to two of the four omnidirectional antennas mounted on the HST carriage, and emulating a mobile passenger terminal directly attached to the LTE network (B2M link), thus enabling one to compare the signals captured by indoor and outdoor receivers. Additionally, each node has been attached with a GPS receiver for geo-referencing (time and position) the measurements. The configured parameters of the measurements are specified in Table 6.1.

Table 6.1. LTE testbed configurations.

\begin{tabular}{l|c}
\hline \hline Item & Description \\
\hline Transmitted power & $46 \mathrm{dBm}$ \\
\hline Carrier frequency & $2.6 \mathrm{GHz}$ \\
\hline Bandwidth & $10 \mathrm{MHz}(9 \mathrm{MHz}$ occupied $)$ \\
\hline Number of subcarriers & $1024(600$ used $)$ \\
\hline Transmitter scheme & Transmit diversity \\
\hline Cyclic prefix length & Normal \\
\hline \hline
\end{tabular}


6. Propagation Inside Mass Transit Systems

\subsubsection{Results Analysis}

For ensuring the safe operation of HST network, the continuous coverage of vital communication service along the tracks is of importance. In the mean time, avoiding the interruption of passenger services and providing an acceptable network access are also raise more and more concern for cellular network deployments. In this section, an empirical study based on the measurements by the LTE testbed as described in Section 6.1.1 is presented. The estimated SNR along the track with an eNodeB transmitting LTE signals is set. The eNodeB provides coverage for two different sectors while both LTE testbed receivers perform a handover approximately when the train passes in front of the tower where the eNodeB antennas are installed. Table 6.1 specifies the main configuration parameters for the LTE testbed and Fig. 6.3 (b) shows the simulated radio coverage of the measurement site.

The SNR is extracted from the RAW data by considering exclusively the data subcarriers. Thus, guard subcarriers, the DC subcarrier as well as pilot subcarriers are discarded a priori. The SNR estimation is conducted by the following steps:

i The noise samples in the time domain are captured with the transmitter switching off (i.e., no signal is transmitted by the eNodeB). In the frequency domain, the noise samples are obtained by FFT processing on the noise samples the time domain as if they are actual data samples (e.g., the cyclic prefix is removed, FFT is performed, and both guard and DC subcarriers are removed).

ii The received data as the combination of the signal and noised samples are obtained when the transmitter is switched on. $r$ denotes the data sample transformed into frequency domain by the similar processing on the noise samples.

iii The average SNR for each OFDM symbol is then estimated by averaging out the instantaneous SNR values for the different data subcarriers. Defining

$$
\bar{r}^{(k)}=\frac{1}{L} \sum_{l=1}^{L}\left|r^{(l, k)}\right|^{2}
$$




\section{Propagation Inside Mass Transit Systems}

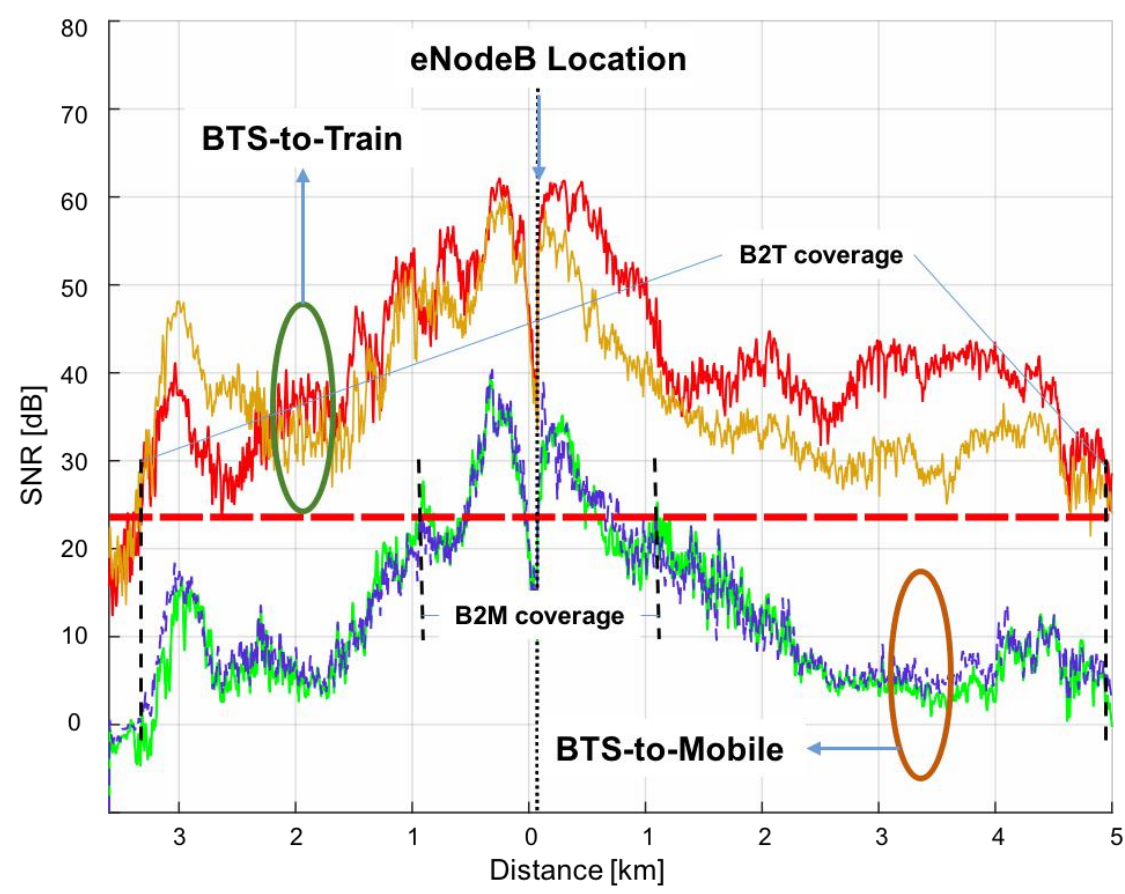

Figure 6.4. Estimated SNR values by means of measurements corresponding to both BTS-Train and BTS-Mobile links.

and

$$
\bar{w}^{(k)}=\frac{1}{L} \sum_{l=1}^{L}\left|w^{(l, k)}\right|^{2},
$$

where $L$ is the number of data subcarriers. $r^{(l, k)}$ and $w^{(l, k)}$ are the received data and noise samples of the $k$-th OFDM symbol at $l$-th subcarrier, respectively.

Consequently, the SNR for each OFDM symbol is calculated as:

$$
\mathrm{SNR}^{(k)}=\frac{\bar{r}^{(k)}-\bar{w}^{(k)}}{\bar{w}^{(k)}}
$$

The estimated SNR values along the moving path of train between KP 93.4 and 102.0 at $50 \mathrm{~km} / \mathrm{h}$ are presented in Fig. 6.4. The upper two of the four curves represent the SNR calculated based on the received samples from two outdoor antennas located on the roof of the train carriage. Then, the SNR of the received samples inside the train is given by the other two curves. The horizontal axis 
in Fig. 6.4 denotes the distance to the eNodeB, while the vertical axis shows the estimated SNR values in $\mathrm{dB}$. When the train carriage of the LTE receivers located passes in front of the BTS tower at KP 97.075 (0 km in Fig. 6.4), where the handover between the two deployed sectors occurs.

As shown in Fig. 6.4, the obtained SNR results of two pairs of outdoor and indoor links are basically consistent. Generally, the SNR values on the external antennas are above $10 \mathrm{~dB}$ for all the considered measurement points, and the maximum values are obtained when the train is proximity to the eNodeB location. Whereas the indoor SNR is approximately $30 \mathrm{~dB}$ lower than the outdoor receiving due to the extra loss generated by the train car. The so-called cell radius is defined as the range where SNR on outdoors and indoors antennas above a threshold value. If this threshold value is set to $25 \mathrm{~dB}$, it can be observed that the cell radius for outdoor antennas is four times larger than for indoor ones.

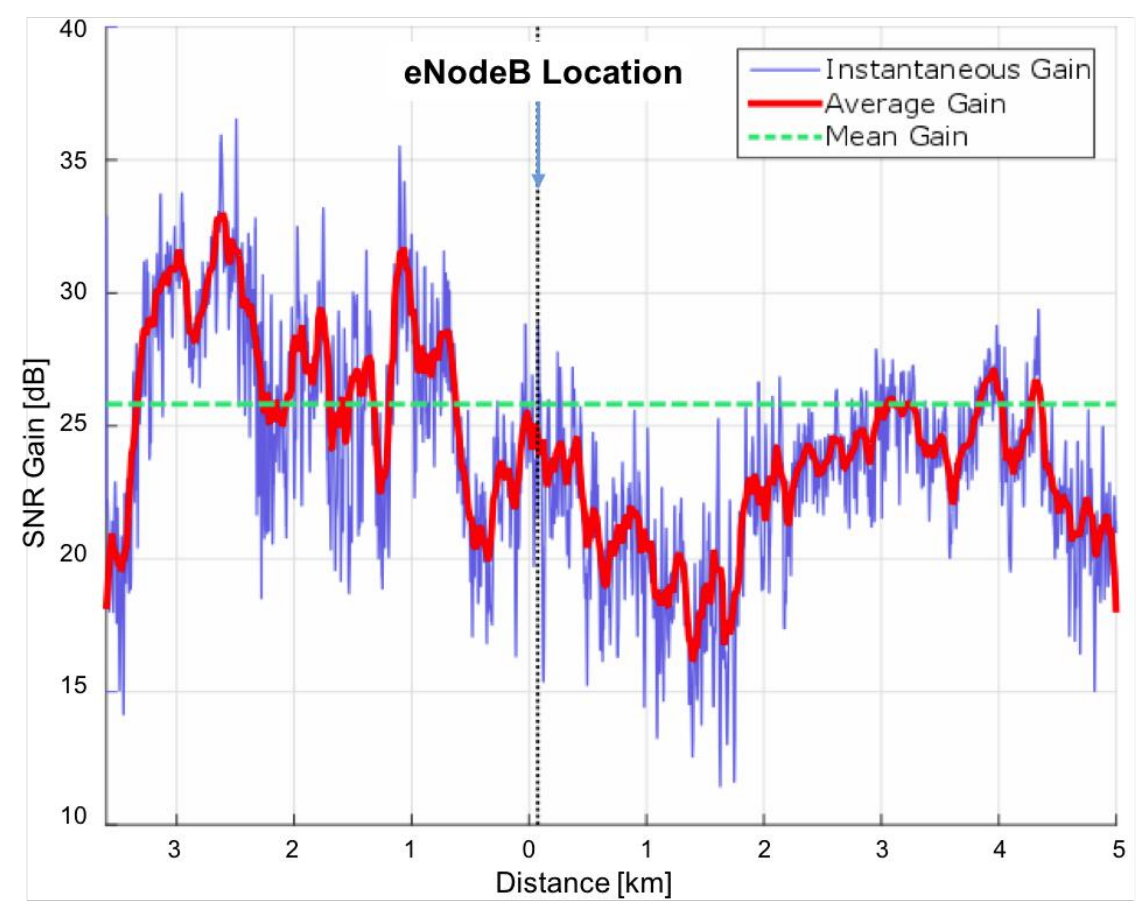

Figure 6.5. Estimated SNR difference between the B2T and B2M links.

Fig. 6.5 shows more clearly the SNR differences between the external and internal antennas. This SNR gain is computed as the difference between the 
6. Propagation Inside Mass Transit Systems

instantaneous SNR values obtained for the outdoor antenna and the indoor one; both expressed in dBs. A curve obtained by averaging the instantaneous values by using a sliding window of $6.5 \mathrm{~ms}$ long is also plotted. It can be seen that, during most of the time, the SNR gain varies between $20 \mathrm{~dB}$ and $30 \mathrm{~dB}$. The average SNR gain for the whole path is also indicated in Fig. 6.5, and it is about $26 \mathrm{~dB}$. This value can be considered as an example penetration loss caused by the moving train carriage on the radio waves at $2.6 \mathrm{GHz}$.

\subsection{Layer II: Propagation inside Trains}

An economical approach to solving the attenuation from train body is the relay system inside the train, which requires very few equipment upgrades. Moreover, broadband access based on the wireless relay techniques can provide not only efficient interconnection with trains monitoring and surveillance systems but also high capacity data services for passengers. For instance, IEEE 802.11.ac offers an overall link minimum of $1 \mathrm{~Gb} / \mathrm{s}$. Furthermore, the new IEEE 802.11.ad standard can deliver data transfer rates up to $7 \mathrm{~Gb} / \mathrm{s}$ within $2 \mathrm{GHz}$ bandwidth at $60 \mathrm{GHz}$ frequency band, which is a potential mmWaves solution for up to 100 users per carriage.

\subsubsection{Measurement Scenarios and Configurations}

A series of propagation measurements onboard the HST have been conducted inside the trains. These measurements have been performed with the train parked at the train station. The interior structure and the cross section of the HST can be found in Fig. 6.6.

The transmitter and receiver testbeds have been configured with two arrangements for three links:

- Station-Mobile link: for modeling the attenuation and propagation onboard the train, in the case of an AP is installed outside the train, e.g., on the platform of the station. With this setup, the transmitter has been placed $3 \mathrm{~m}$ away in front of a train's door. Moreover, this link is tested at both 2.4 


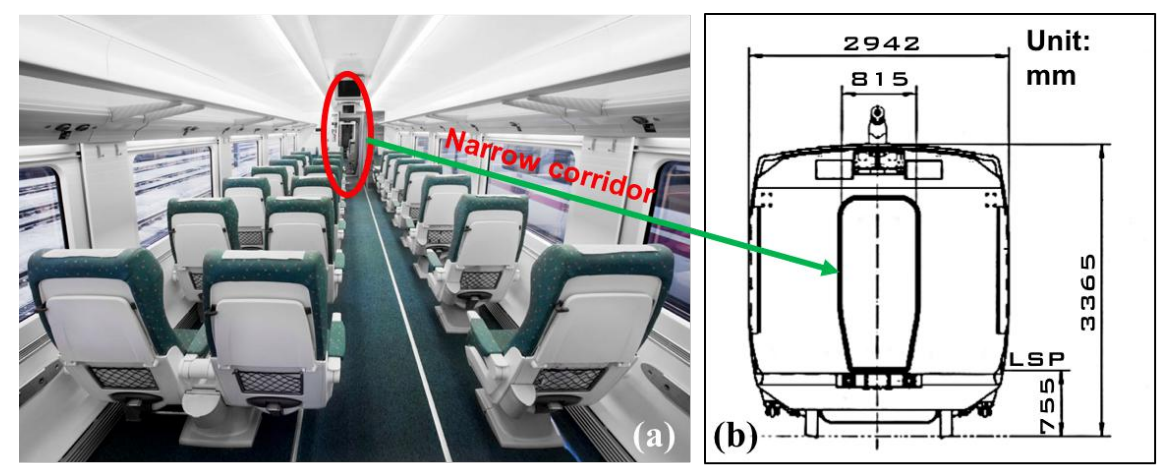

Figure 6.6. Indoor test environments: (a) interior of the commercial train car (via: www.talgo.com); (b) cross section of the test train.

$\mathrm{GHz}$ and $5.7 \mathrm{GHz}$. In the mean time, the receiver has been moved inside the train carriage.

- Intra-Carriage and Inter-Carriage links: for evaluating the path loss and fading inside the train carriage, the transmitter has been installed in the tail of the first carriage with a height of $1.4 \mathrm{~m}$, and the receiver has been moved from the head of the first carriage to the next carriages. The transmitter is assumed as the onboard access point working at $2.4 \mathrm{GHz}$.

\section{Testbed}

The propagation testbed transmitter consists of three CW transmitters followed by power amplifiers and antennas for each considered carrier frequency (see Fig. 6.7 (a)). Propagation testbed configuration parameters, such as transmit power and antenna gain at each frequency, are listed in Table 6.2.

At the receiver side, the testbed consists of a portable spectrum analyzer with pre-amplifier as shown in Fig. 6.7 (b). All these devices are controlled by customdeveloped software installed in a laptop computer. Moreover, two omnidirectional antennas performed at $2.4 \mathrm{GHz}$ and $5.7 \mathrm{GHz}$ are attached on the shoulder of the operator and connected to the receiver. Measurements are conducted at both frequencies separately. The moving distance of the receiver is about $40 \mathrm{~m}$, which is approximately equal to the length of two train carriages. 

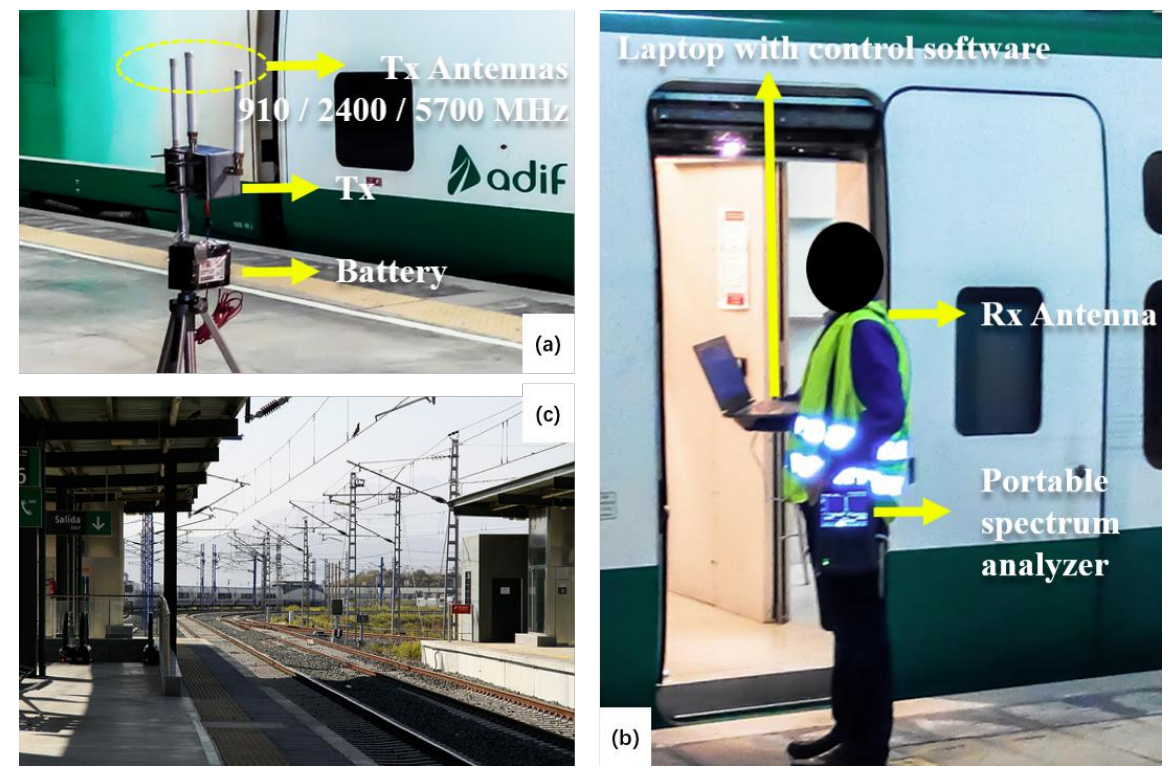

Figure 6.7. Photographs and Setups of the testbed transmitter and receiver.

Table 6.2. Parameters of the testbed transmitter.

\begin{tabular}{c|c|c|c}
\hline \hline Frequency & Tx Power & Antenna Gain & EIRP \\
\hline $910 \mathrm{MHz}$ & $26.0 \mathrm{dBm}$ & $3.0 \mathrm{dBi}$ & $29.0 \mathrm{dBm}$ \\
\hline $2.4 \mathrm{GHz}$ & $28.8 \mathrm{dBm}$ & $5.0 \mathrm{dBi}$ & $33.8 \mathrm{dBm}$ \\
\hline $5.7 \mathrm{GHz}$ & $19.5 \mathrm{dBm}$ & $6.5 \mathrm{dBi}$ & $26.0 \mathrm{dBm}$ \\
\hline \hline
\end{tabular}

\subsubsection{Result Analysis \& Channel Characterization}

\section{Station-Mobile Link}

Besides the LTE radio coverage in the open area, the propagation from the station into the train carriage is another interesting point. Assuming an AP installed in the train station, and the mobile device receives the signal inside the train. In this case, the radio waves are attenuated by the train body and continue to propagate inside the train carriage. To model this effect, a corresponding measurement has been conducted as illustrated in Fig. 6.8. During the measurement, 


\section{Propagation Inside Mass Transit Systems}

the train is motionlessly stopped inside an HSR station. The transmitter has been placed in front of the second car and kept 3m from the train's door. The receiver has been attached to the operator, who has moved inside the train along the passage of the first two cars as indicated by the red arrow in Fig. 6.8. Then the operator has stepped out and took reference level near the train's door and then re-enter the train. The same process has been repeated at both $2.4 \mathrm{GHz}$ and 5.7 GHz.

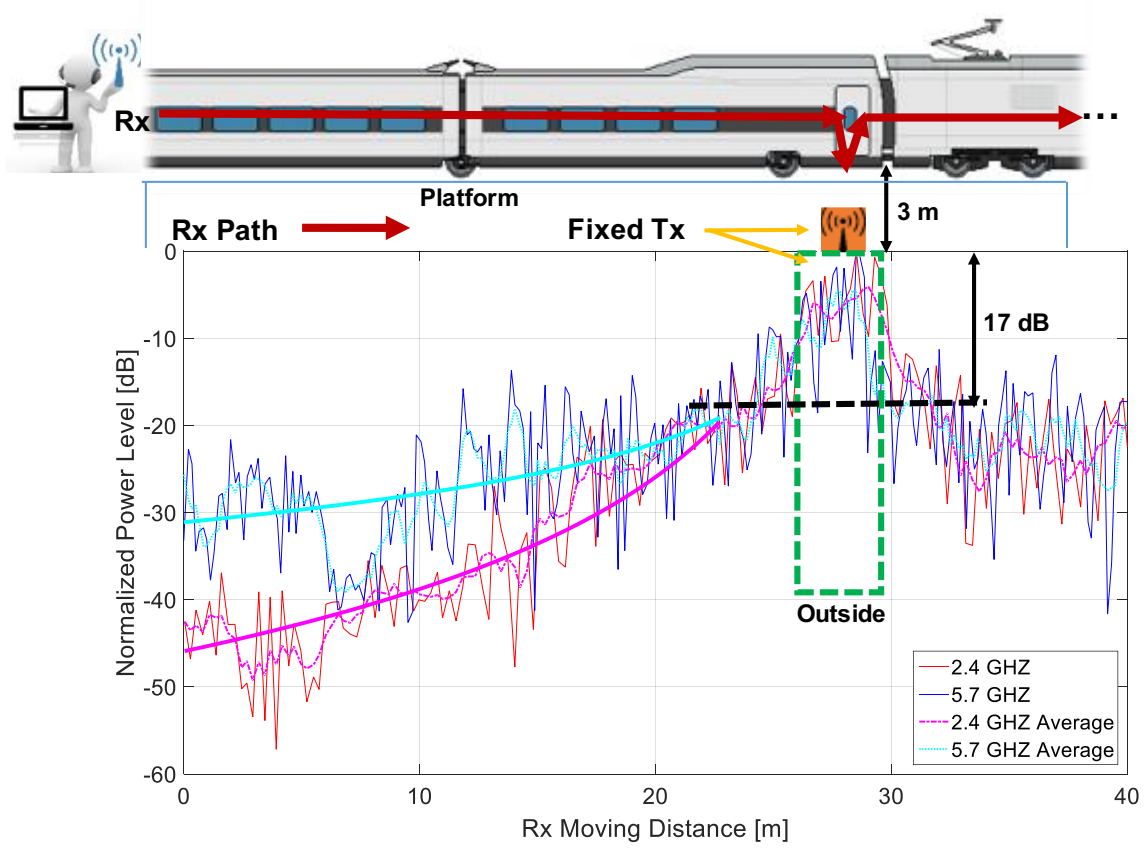

Figure 6.8. Received signal power level of Station-Mobile link at $2.4 \mathrm{GHz}$ and 5.7 $\mathrm{GHz}$.

The normalized received power level is also given in Fig. 6.8. As the distance between transmitter and receiver decreases, the power level increases at both frequencies. When the operator approaches to the transmitter and near the door, the received power level shows a dramatical increasing, due to the significant LOS propagation occurs. A noticeable power level difference about $17 \mathrm{~dB}$ from outside to inside the train is marked. This value can be considered as the penetration loss in a semi-closed train station as shown in Fig. 6.7 (c). It is worth noting that propagation at $5.7 \mathrm{GHz}$ experienced less attenuation than at $2.4 \mathrm{GHz}$. A similar conclusion can be found in [211]. 


\section{Intra-Carriage and Inter-Carriage}

Propagation inside the train is rarely measured in a real environment. Nevertheless, the deployment of a relay system in the train or another MTS requires knowledge of the wireless channel inside these systems. The channel measurement in the case of transmitter and receiver onboard the train has been conducted as shown in Fig. 6.9. The testbed transmitter has been installed on a seat of the first train carriage at $1.4 \mathrm{~m}$ height. Similarly, the receiver has been moved along the passage way of the train. Note the moving path in Fig. 6.9 is divided into two parts as the red and green arrows denoted, which represent the Intra-Carriage path and Inter-Carriage path, respectively. Also, the normalized received power level along the moving path is also plotted in Fig. 6.9. It can be seen that the received power level increases slowly as the receiver moves toward the transmitter and reaches its peak value when the receiver passes by the transmitter. Then a large attenuation more than $20 \mathrm{~dB}$ is observed within the narrow corridor between carriages because the inter-door between the carriage door is noticeably narrower than the rest of the train structure (see Fig. 6.6(b)), thereby obstructing the received signal. This effect is also reported in [213] as a shielding loss of about $20 \mathrm{~dB}$. When the operator arrives at the next train carriage, the multiple reflected waves from the surrounding environment (see Fig. 6.7(c)) result in the power level rising back, but globally maintaining a downward trend as the receiver moves away the transmitter. Thus, the complete link can be divided into two parts: Intra-Carriage link and Inter-Carriage link, which are consistent with the moving paths of the receiver.

Besides the HST, rapid transit, also known as metro, subway, is another widely used MTS around the world, which is an another type of high-capacity public transport generally found in urban areas. Thus, the propagation inside the metro train is worth studying. As shown in Fig. 6.10, the received power level along the carriages is also collected, which also can be separated by Intra-Carriage and Inter-Carriage links. Unlike the propagation measurements inside HST, the receiver has constantly been moved away the transmitter. In addition, the metro train is not sealed as HST, due to the operation speed. The internal structures of the metro train and HST are also different. Morden metro train is usually 


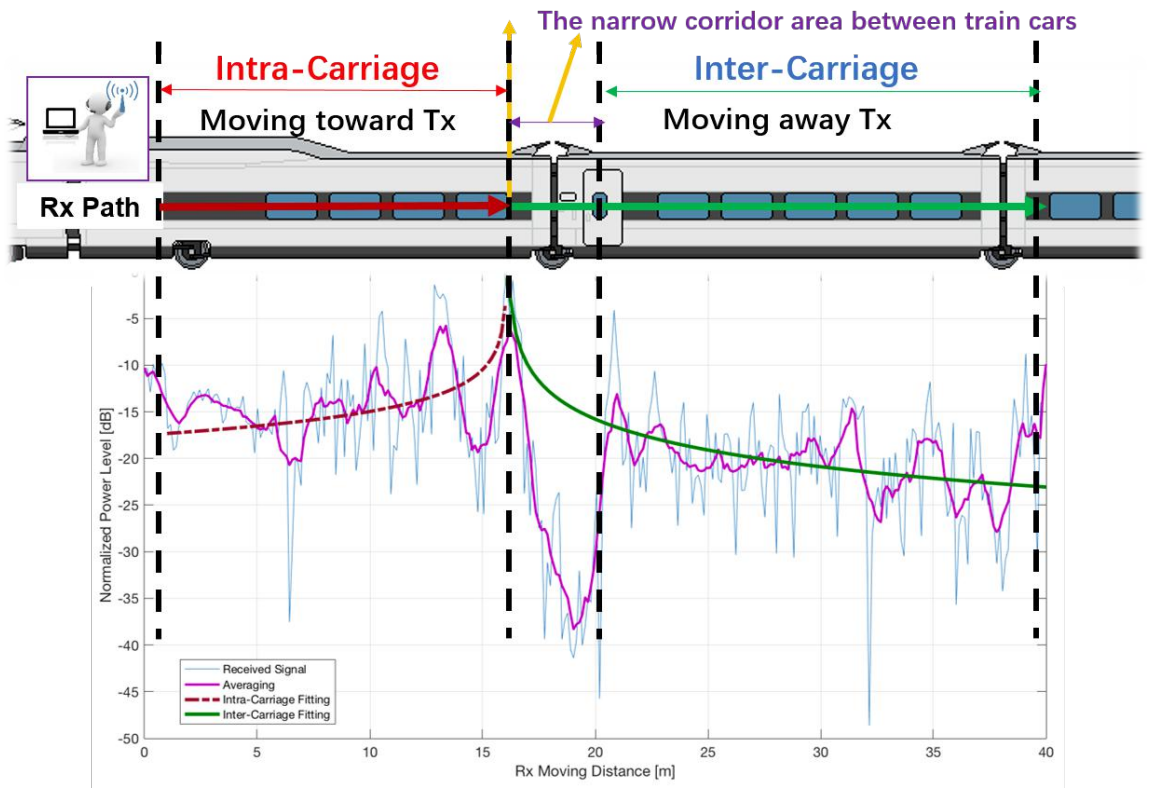

Figure 6.9. Received signal power level of the Intra-Carriage and Inter-Carriage links inside the HST.

designed to an open corridor without a door between the carriages to make easier for the circulation of passengers. This difference results in different behaviors of the radio waves propagated inside the trains. For example, the large attenuation caused by the narrow corridor and inter-door between the carriages of HST is hard to observe in Fig. 6.10. As the receiver moves away from the transmitter in the same carriage to the next carriages, the received power level presents different decay rates.

\section{Large-scale Fading Characterizations}

Propagation is affected by the distance-dependent attenuation plus the largescale shadowing and small-scale fading. The large-scale shadowing is the result of large obstructions, and the small-scale fading arises due to the constructive and destructive combination of multipath components. Thus, to separate the largescale and small-scale fading, channel gain is averaged over ten wavelengths. For obtaining the propagation model inside the train, the narrow corridor with large shielding loss is not considered during the fitting process in Fig 6.9. As the log 


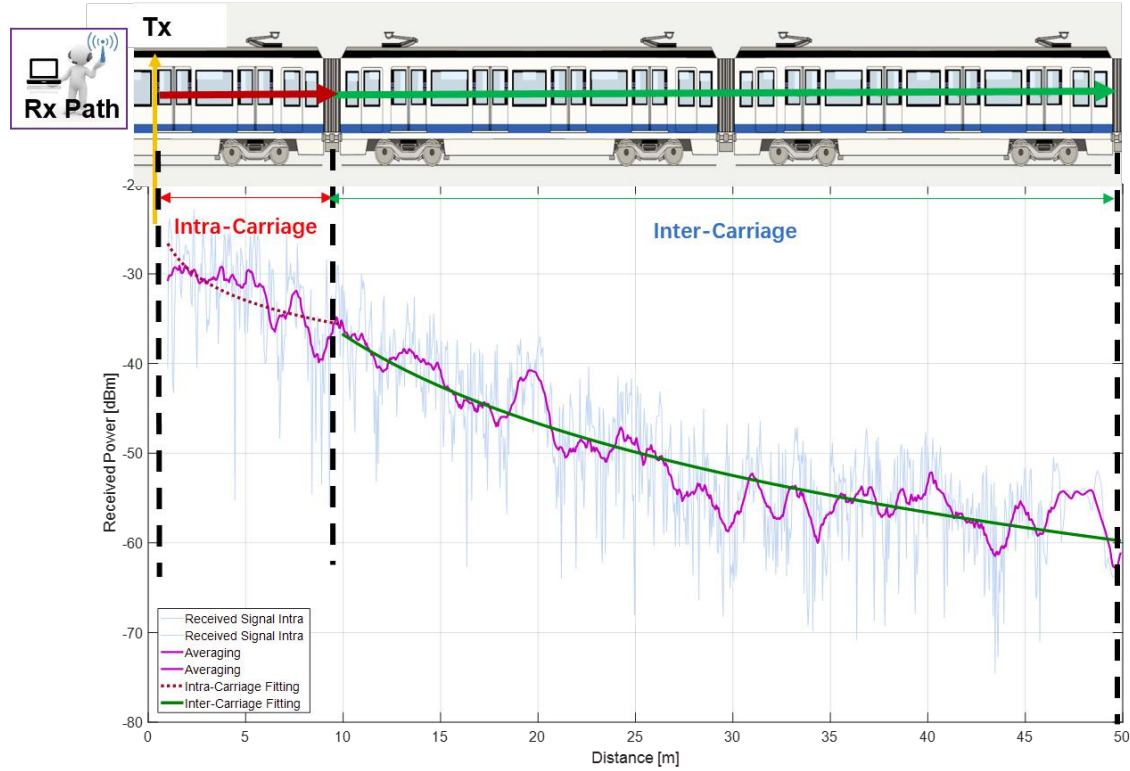

Figure 6.10. Received signal power level of the Intra-Carriage and Inter-Carriage links the metro train.

distance path loss model discussed in Section 2.2.1, the path loss in decibels (dB) is found as the difference between transmitted power $P_{T} x$ and received power $P_{R} x$, and the loss is modeled versus the logarithm of distance as follows:

$$
P_{L}(d)=P_{\mathrm{Tx}}-P_{\mathrm{Rx}}=P_{0}+10 n \log _{10}\left(\frac{d}{d_{0}}\right)+X_{s}
$$

Where $d_{0}$ is the close-in reference distance, $P_{0}$ is the fit intercept at $d_{0}$, and $n$ is the PLE, which describes the path loss trend with respect to the link distance d. Variable $X_{s}$ represents a zero mean Gaussian random variable in dB with a standard deviation $\sigma_{x}$.

Through least-squares regression fittings as Fig.6.11 and Fig.6.12 show, the PLE $n$ and the standard deviation $\sigma_{x}$ of the variations can be estimated. These two key parameters for large-scale fading characterization in Intra-Carriage, InterCarriage, and Station-Mobile links are listed in Table 6.3. As the comparisons, $n$ and $\sigma_{x}$ extracted from the measurement results in similar scenarios, such as inside airplanes, tunnels, indoor corridors, and propagation from outdoor-to-indoor settings, are listed in Table 6.3. It is widely knowledged that PLE $n$ in free space is equal to 2 . However, $n$ is less than 2 in both Intra-Carriage and Inter-Carriage 


\section{Propagation Inside Mass Transit Systems}

links of HST, which is similar to the cases of inside small aircraft, tunnels, and corridors. These kinds of confined environments always produce a guiding effect on the signal, yielding an exponent less than 2. Furthermore, our $n$ is smaller than the values typically obtained from corridors and subway tunnels. This is because the steel body of the train has higher reflectivity than brick or concrete walls, and this strengthens the waveguide effect. The relatively small exponents have been observed in [213] and [214] on board the trainbut our smaller $n$ might because of more metallic equipment and more crowded inner space. Next, the larger $n$ in the Inter-Carriage link than in the Intra-Carriage link predicts a higher path loss in the following train cars because of the changes of train's structure. In the meantime, the measurement result of Intra-Carriage in metro train also observes a small $n$ of 0.93 , due to the spacious internal structure of metro train with respect to the HST. Since the decay distance of Inter-Carriage in the metro train is large enough, the larger PLE $n$ of 3.3 is achieved. Thus, the path loss in the metro train can be considered as a dual-slope model with two PLEs.

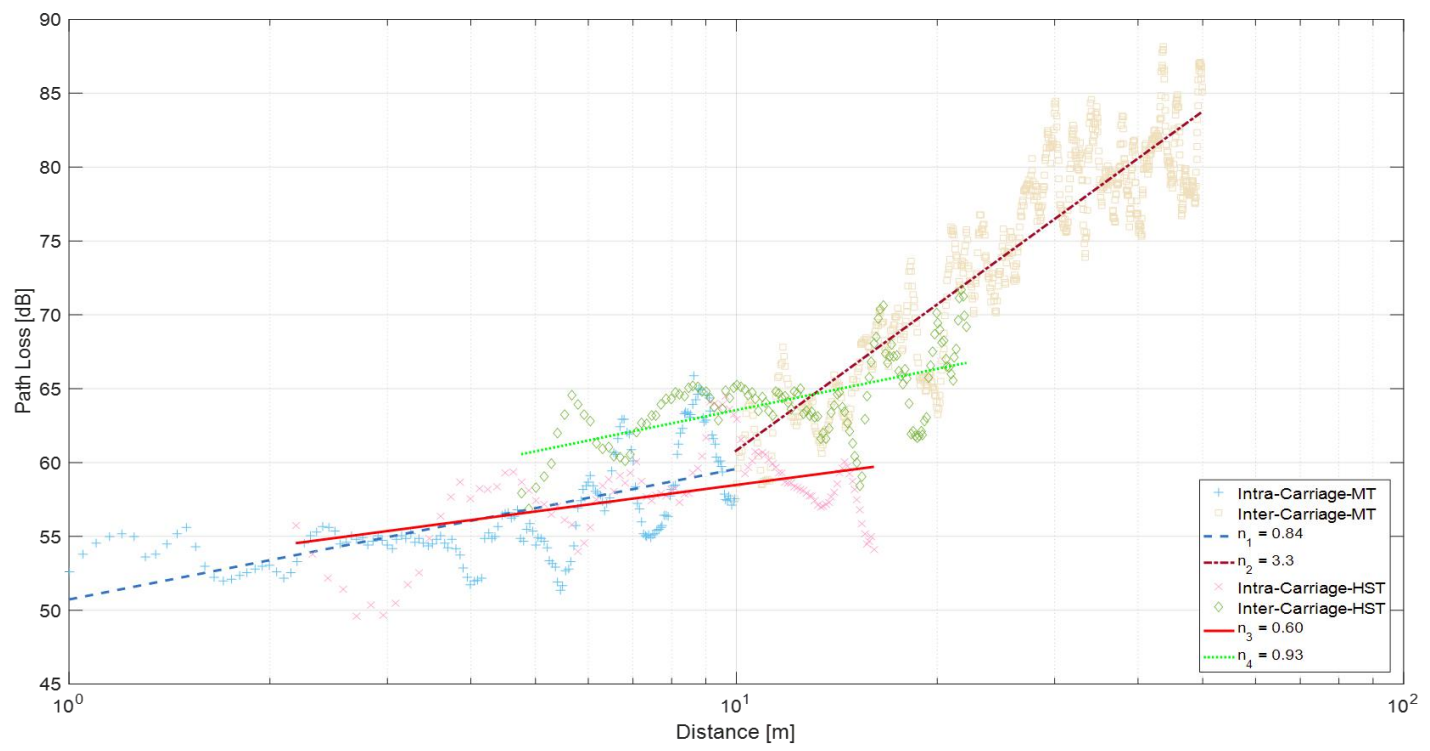

Figure 6.11. Path loss and least-square fittings for Intra-Carriage and InterCarriage links inside HST and Metro train.

When the transmitter is located outside the train, the transmitted waves penetrate the train car body and continue to propagate inside the train. Thus the propagation is partially affected by waveguiding. As waveguide theory shows, 


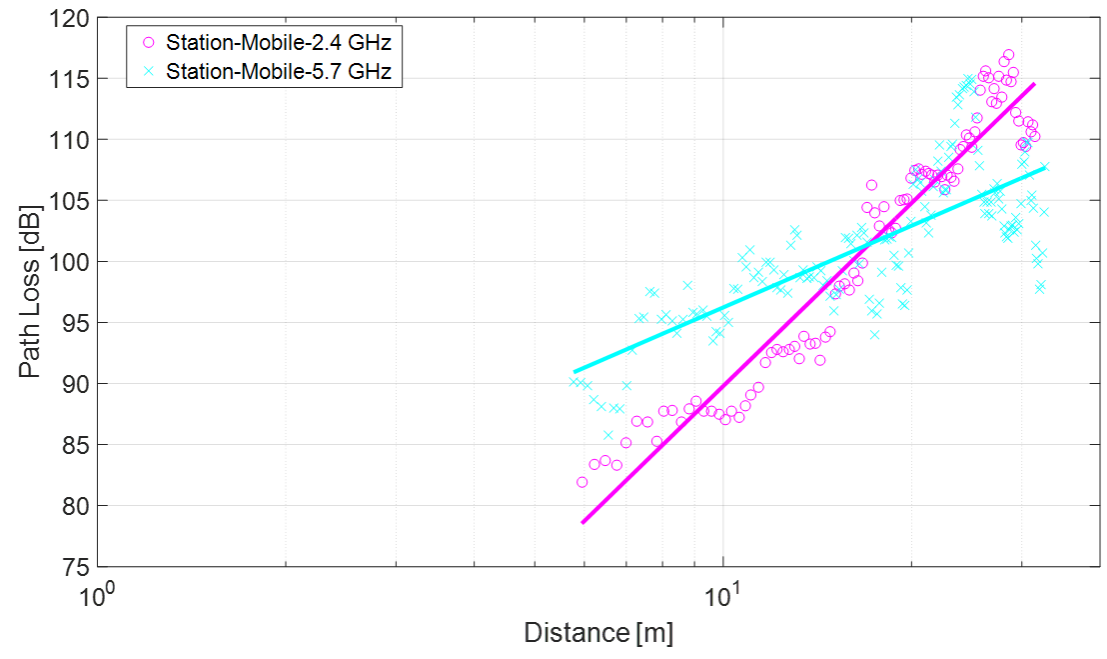

Figure 6.12. Path loss and least-square fittings for Station-Mobile links inside HST.

waves at higher frequencies experiences smaller propagation loss [211]. Thus, $n$ is larger at $2.4 \mathrm{GHz}$ than at $5.7 \mathrm{GHz}$.

For selecting the best fitting model to describe the small-scale fading, Akaike's Information Criteria (AIC) has been introduced in [221]. AIC is a goodness of fit measure that favors smaller residual error in the model. The general form for calculating AIC is as follows:

$$
A I C=-2 \ln (\text { Likelihood })+2 U
$$

where $\ln$ is the natural logarithm, (Likelihood) is the likelihood function of the associated distribution, and $U$ is the number of free parameters in the model. AIC scores are often evaluated by the AIC difference, which is given by:

$$
D_{j}=A I C_{j}-\min _{i}\left(A I C_{i}\right)
$$

where $\min _{i}\left(A I C_{i}\right)$ is the minimum AIC over $J$ distribution models.

Therefore, the best model has a $D$ of 0 . Furthermore, the Akaike weights can be introduced as conditional probabilities for each mode, and greatly facilitate the comparisons among the candidate models. [222]. The $j^{\text {th }}$ Akaike weights $w_{j}$ is given by: 
6. Propagation Inside Mass Transit Systems

Table 6.3. Comparison for path loss model factors in Intra-Carriage, InterCarriage, Station-Mobileand from the literature.

\begin{tabular}{cc|cc}
\hline \hline Scenario & Frequency $[\mathrm{GHz}]$ & PLE $n$ & Std. dev. $\sigma_{x}[\mathrm{~dB}]$ \\
\hline Intra-Carriage HST & 2.4 & 0.60 & 2.59 \\
\hline Intra-Carriage Metro & 2.6 & 0.84 & 2.65 \\
\hline Inter-Carriage HST & 2.4 & 0.93 & 2.34 \\
\hline Inter-Carriage Metro & 2.6 & 3.3 & 2.93 \\
\hline Station-Mobile & 2.4 & 3.81 & 2.93 \\
\hline Large Aircraft [198] & 5.7 & 1.74 & 3.85 \\
\hline Small Aircraft [199] & $1.8-2.45$ & $2.0-2.7$ & $3.7-5.3$ \\
\hline Indoor Corridor [209] & $3.1-10.6$ & $0.20-1.52$ & $0.29-2.44$ \\
\hline Subway Tunnel [210] & 5.25 & 1.8 & 3.10 \\
\hline Outdoor-to-indoor [211] & 2.65 & 1.94 & 5.47 \\
\hline Intra-Carriage [213] & $2-6$ & 2.27 & 7.00 \\
\hline Onboard Train [214] & 2.2 & $\approx 1.2$ & - \\
\hline \hline
\end{tabular}

$$
w_{j}=\frac{\exp \left\{-\frac{1}{2} D_{j}\right\}}{\sum_{i=1}^{J} \exp \left\{-\frac{1}{2} D_{i}\right\}}
$$

where $\sum_{j=1}^{J} w_{j}=1$. Akaike weights provide a straightforward interpretation as the probabilities of each models being the best model in an AIC sense. Therefore the model has highest Akaike weights is usually the preferred model.

Table 6.4. Akaike weights of four distribution candidates, and the fitted shape factors of Nakagami and Weibull distribution

\begin{tabular}{l|c|c|c|c|c}
\hline \hline Link & Frequency & Rayleigh & Rician & Nakagami & Weibull \\
\hline Intra-Carriage HST & $2.4 \mathrm{GHz}$ & 0.0193 & 0.0430 & 0.8014 & 0.1362 \\
Inter-Carriage HST & $2.4 \mathrm{GHz}$ & 0.0928 & 0.1447 & 0.4836 & 0.2789 \\
\hline Intra-Carriage Metro & $2.6 \mathrm{GHz}$ & 0.0624 & 0.2776 & 0.5083 & 0.1517 \\
Inter-Carriage Metro & $2.6 \mathrm{GHz}$ & 0.0000 & 0.0002 & 0.9655 & 0.0343 \\
\hline \multirow{2}{*}{ Station-Mobile } & $2.4 \mathrm{GHz}$ & 0.0442 & 0.0862 & 0.6214 & 0.2481 \\
& $5.7 \mathrm{GHz}$ & 0.1492 & 0.0549 & 0.6234 & 0.1725 \\
\hline \hline
\end{tabular}

To model the small-scale fading, although Rayleigh and Rician distributions 
6. Propagation Inside Mass Transit Systems

Table 6.5. Shape factors and K-factors for small-scale characterizations.

\begin{tabular}{l|c|c|c|c|}
\hline \hline Link & Frequency & Nakagami $m$ & Weibull $\beta$ & K-factor $[\mathrm{dB}]$ \\
\hline Intra-Carriage HST & $2.4 \mathrm{GHz}$ & 1.4914 & 2.3898 & 1.2952 \\
Inter-Carriage HST & $2.4 \mathrm{GHz}$ & 1.2974 & 2.2946 & -0.3689 \\
\hline Intra-Carriage Metro & $2.6 \mathrm{GHz}$ & 1.2068 & 2.2893 & -1.5097 \\
Inter-Carriage Metro & $2.6 \mathrm{GHz}$ & 1.2926 & 2.2906 & -0.4207 \\
\hline \multirow{2}{*}{ Station-Mobile } & $2.4 \mathrm{GHz}$ & 1.4444 & 2.4108 & 0.9537 \\
& $5.7 \mathrm{GHz}$ & 1.2536 & 2.1881 & -0.8755 \\
\hline \hline
\end{tabular}

have been proposed and performed well in describing multipath fading statistics in many cases, other models, such as the Nakagami and Weibull distributions are considered as generalized alternative solutions; they include the Rayleigh as a special case and can be more accurate in matching some experimental data [223] [224]. Therefore, the received signal amplitudes (large-scale fading removed) are fitted with these four empirical distributions. The Akaike weights of these distribution models are listed in Table 6.4. Results clearly show that the Nakagami is the best fit model with highest Akaike weights for all links, and the Weibull is the second best. In addition, the shape factors $m$ and $\beta$ are provided in Table 6.5. Also, based on the values of $m$, the corresponding Rician K-factors are calculated by Eq. 2.11. Fig. 6.13 shows the plotting of the empirical cumulative distribution functions (CDFs) against the CDFs of Nakagami distribution. In the special case of $m=1$ or $\beta=2$, Rayleigh fading pertains and implies the signal offered to the receiver does not contain a dominative wave. Whereas in our cases, $m$ and $\beta$ are greater than 1 and 2 , respectively. The Rayleigh distribution has the lowest Akaike weights in all links. As one would expect, even though there are many reflection and scattering components inside the train station and train car, the fading in the Intra-Carriage, Inter-Carriage, and Station-Mobile links is not as severe as in a Rayleigh channel, and it can be described as a propagation channel with a dominant component.

Additionally, the multiple reflected waves outside the train can re-enter the train carriage through the windows and interfere with the wave propagating inside the trainthus lead to compensation on the path loss. As the authors in [217] state, if the windows have been covered with absorbers, there has been a $10.4 \mathrm{~dB}$ 


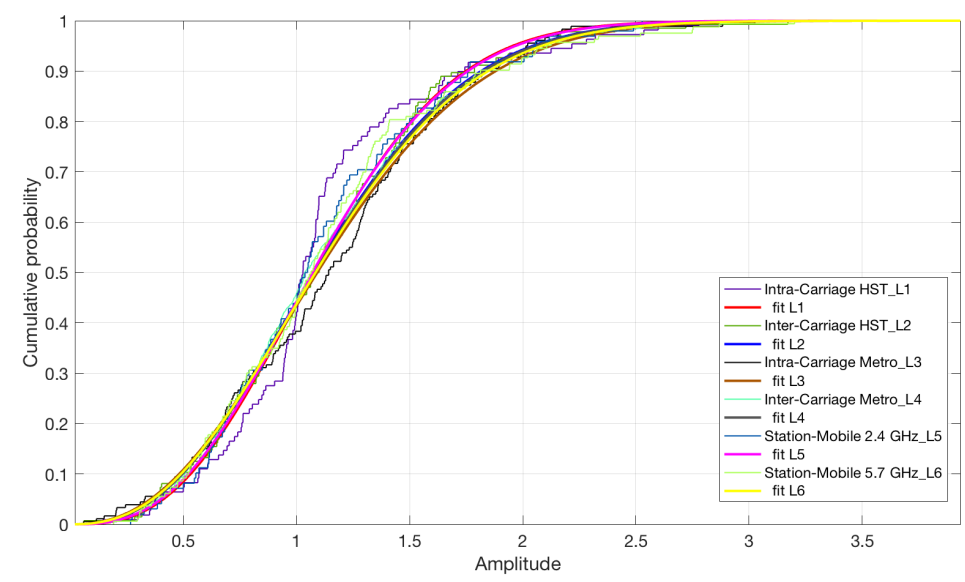

Figure 6.13. Nakagami distribution fitting with small-scale amplitude fading statistics of wireless links.

extra path loss at $5.2 \mathrm{GHz}$. In our case, the windows are made of heat protective glass and covered an absorber layer to attenuate sunlight, and this layer can also attenuate communications signals. There is a total attenuation of $40 \mathrm{~dB}$ imparted to the re-entering signals as reported in [225], which has assumed the fast moving scatters outside the train do not affect considerably to the propagation. The extra loss of windows may be different for different trains and different environments in which the train is operated induce a different amount of "re-entering"; these are topics worthy of further research.

\section{Angle of Arrival}

The Angle of Arrival (AoA) of the received signal also severely affects the path loss. The AoA depends on the relative angle between the BTS/AP antennas and the train. Since the BTS antennas are typically installed in the towers close to the track, the AoA magnitude is small at most of the time, which increases the attenuation level, and achieving the minimum attenuation value when the train passes in front of the transmitter antennas $\left(A o A=90^{\circ}\right)$. For evaluating this effect, an additional measurement has been conducted. The propagation testbed transmitter has been moved parallel to the train from $90^{\circ}$ (i.e., in front of the fixed testbed receiver) to the head of the train, as shown in Fig. 6.14. 
The PLE $n_{a}$ is described as the change of the path loss with respect to the AoA. Therefore, propagation loss as a function of the AoA can be written as

$$
L(\alpha)=L\left(\alpha_{0}\right)+10 n_{a} \log \left(\frac{\alpha}{\alpha_{0}}\right)
$$

where $\alpha$ is the AoA, $\alpha_{0}$ is a reference AoA value, and $L\left(\alpha_{0}\right)$ is the average path loss at the point of the reference AoA $\alpha_{0}$.

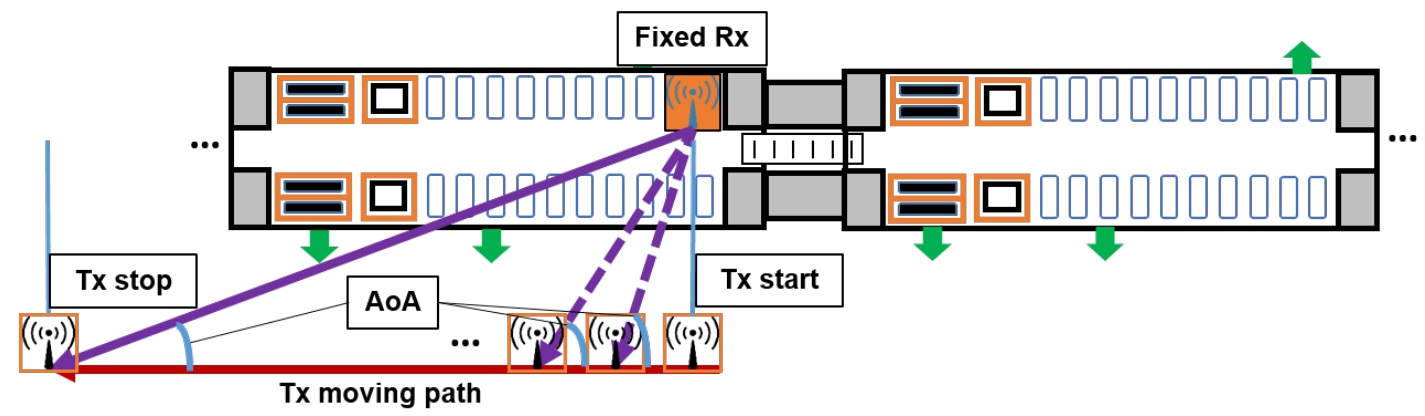

Figure 6.14. Measurement setup for testing the influence of the AoA.

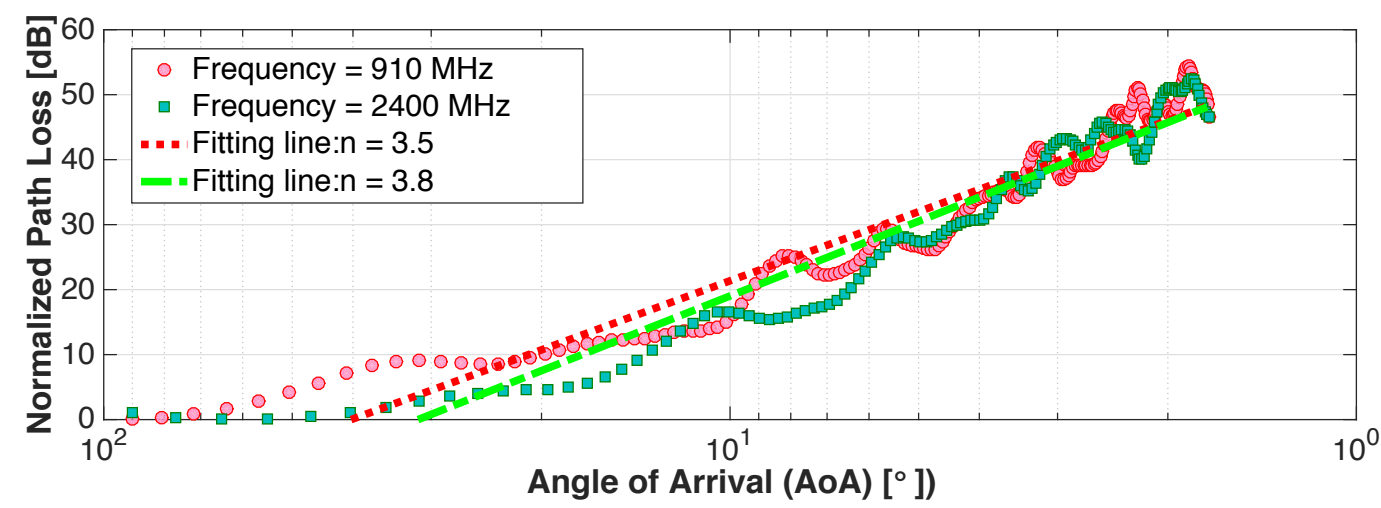

Figure 6.15. Normalized path loss versus AoA.

Fig. 6.15 shows the normalized path loss as a function of the AoA obtained through the measurements. The normalized path loss 0 represents the minimum separation between $\mathrm{Tx}$ and $\mathrm{Rx}$ that the AoA is $90^{\circ}$. Measured data is fitted by applying LS regression, and the slope of each curve is used to compute the PLEs, while $\alpha_{0}$ is computed as the minimum AoA value such that $L\left(\alpha_{0}\right) \approx 0$. Table 6.6 
6. Propagation Inside Mass Transit Systems

Table 6.6. PLE characterization as a function of the AoA.

\begin{tabular}{c|c|c}
\hline \hline Frequency & AoA Intercept $\alpha_{0}$ & PLE $n_{a}$ \\
\hline $910 \mathrm{MHz}$ & $40^{\circ}$ & 3.5 \\
$2400 \mathrm{MHz}$ & $31^{\circ}$ & 3.8 \\
\hline \hline
\end{tabular}

specifies the obtained PLEs as well as the $\alpha_{0}$ values for the different carrier frequencies. It can be seen that signals at the lowest frequency of $910 \mathrm{MHz}$ start to be noticeable attenuated for AoA values $\left(\alpha_{0} \approx 40^{\circ}\right)$ larger than those corresponding to the highest frequency of $2400 \mathrm{MHz}\left(\alpha_{0} \approx 31^{\circ}\right)$. However, for AoA values lower than $\alpha_{0}$, the slope of the curve increases with the carrier frequency. Note that for AoA values close to $1^{\circ}$, the attenuation level exceeds $50 \mathrm{~dB}$ for both $910 \mathrm{MHz}$ and $2400 \mathrm{MHz}$ carrier frequencies.

\subsection{Discussion}

This chapter mainly focuses on the propagation in Mass Transit Systems. Based on the channel measurement and modeling onboard the HST and metro train, the following conclusions can be summarized:

- The train carriage structure introduces a strong signal penetration loss. The amount of penetration loss has been grossly estimated both by evaluating the difference between BTS-to-Mobile and the BTS-to-Train links via a standard-compliant LTE signal received by a moving train. For LTE signals received inside the train, the average attenuation observed with the moving train has been approximate $20 \mathrm{~dB} \sim 30 \mathrm{~dB}$ at $2.6 \mathrm{GHz}$. This large attenuation reduces the cell radius of radio coverage from $8.5 \mathrm{~km}$ with external antennas to $2 \mathrm{~km}$ with internal antennas. When the train is statically parked in the HSR station, an example value of $17 \mathrm{~dB}$ as the penetration loss is observed by the mean of Station-Mobile link measurement.

- As path loss models for the propagation inside the train reveal, IntraCarriage and Inter-Carriage links at $2.4 \mathrm{GHz}$, and Station-Mobile links at 


\section{Propagation Inside Mass Transit Systems}

5.7 GHz are influenced by waveguiding effects, which result in the PLEs less than 2. Attenuation along the train is lower than that of free space except for the narrow corridor between the carriages. Due to the different train structure and decay distance, path loss in the metro train can be characterized as a dual-slope model, which has a small PLE (0.93) for Intra-Carriage link and a PLE larger than 2 for Inter-Carriage link. In small-scale, the received signal amplitudes of all links show good fittings with Nakagami and Weibull distribution with $m>1$ and $\beta>2$, respectively. This implies the presence of dominant component for the Intra-Carriage, Inter-Carriage, and Station-Mobile links.

- The influence of AoA depended on the relative angle between the BTS/AP antennas and the train is evaluated in this chapter. As the AoA decreases from $90^{\circ}$ to $1^{\circ}$, the path losses at $910 \mathrm{MHz}$ and $2400 \mathrm{MHz}$ increase with rates of 3.5 and 3.8 , respectively.

Hence, to provide a reliable communications systems for MTS passengers with reasonable cell sizes, it is necessary to the use a relay based solution with external and internal antennas. The signal inside the train cars can be easily distributed by using $\mathrm{WiFi}$ access points or directly using repeaters. 2.4 or $5.7 \mathrm{GHz}$ can be proper carrier frequencies for signal distribution inside train carriages and also suitable for Inter-Carriage propagation. 


\section{Chapter 7}

\section{Conclusion \& Future Work}

In this thesis, a series of propagation measurements have been described in various complex environments. Corresponding studies on the channel characterization and modeling have been conducted based on the measurement results with different configurations. In this chapter, the main conclusions are summarized, and the related issues for future work are discussed as well.

\subsection{Conclusion}

First, the comprehensive propagation measurements are conducted in the curved traditional arched and modern semicircle subway tunnels with $300 \mathrm{~m}$ and 500 $\mathrm{m}$ radius of curvature, horizontal and vertical polarizations, at $920 \mathrm{MHz}, 2400$ $\mathrm{MHz}$, and $5705 \mathrm{MHz}$, respectively. The extensive measurements in curved subway tunnels provide the insight into the large-scale fading characteristics in real underground environments at various frequencies. The qualitative analysis parameterizes the log-distance path loss models and characterizes the autocorrelation, decorrelation distances, and cross-correlation of shadow fading. These empirical findings are useful for the radio coverage, link budget, and simulation validation of various wireless communication systems deployed in real curved tunnels.

Then, wideband communication measurements in a modern and realistic subway station and tunnel are presented. The classical modeling approach based on the time delay spread, as well as the electromagnetic reverberation theory are employed in channel modeling. The RMS delay and reverberation time are extracted from the empirical results and combined to characterize the channel 
in detail. The subway station covered with steel panels is considered as a dense multipath environment, which is observed longer RMS delay and reverberation time than in the tunnel. Propagation at $2450 \mathrm{MHz}$ is proved to have much fewer MPCs than at $980 \mathrm{MHz}$. Also, the movement of the train in the station has limited influence on the delay spread. Moreover, the transition region between tunnel and station has been defined and modeled based on the Q factors along the distance. In this region, MPCs are gradually reduced by the filtering effect of the tunnel. The transition region is over $720 \mathrm{~m}$ long at $980 \mathrm{MHz}$, whereas this distance is only $350 \mathrm{~m}$ at $2450 \mathrm{MHz}$. In summary, the use of $2.4 \mathrm{GHz}$ or higher frequencies and the location of antennas inside tunnel sections next to the station, can considerably reduce the multipath effects in underground environments.

For evaluating the channel dispersion in high mobility scenarios, the wideband propagation measurements are conducted in an HSR station and composite regions include tunnel portal, cutting, and viaduct. The parameters of time delay, including the mean excess delay, the RMS delay spread and the number of channel taps are calculated in the HSR environment. Based on the experimental data, the propagation behaviors in LOS and NLOS regions of the HSR station are analyzed and compared. Furthermore, the TDL channel models can be established for each region in HSR composite scenario. The analysis results reveal some important appearances for this composite scenario channel: the difference of RMS delay at two frequencies is very small while the propagation loss is more serious, the cutting and tunnel portal result in greater RMS delay and the MPCs in viaduct is more influenced by the both ends of cuttings. Moreover, the deterministic channel model for the composite scenario is proposed using the 3D ray tracing method. The modeling results are validated based on the empirical data gathered in the same scenario. The transition regions are explicitly recognizable by the stochastic analysis on small-scale fading and Doppler spread characteristics in the frequency domain. These observations prove the interferences of the scattering components from cuttings create not only deeper fading but also delay and Doppler dispersion. As HSR composite scenario propagation channels have a strong impact on the coverage, reliability and quality of service of HSR mobile communication networks, the results and models are helpful for the HSR communication system design, network planning, and optimization. 
Finally, the train carriage structure leads to a significant signal penetration loss as the LTE radio coverage testing reveals. The amount of penetration loss is grossly estimated by evaluating the difference between BTS-to-Train and the BTS-to-Mobile links via a standard-compliant LTE signal received onboard a moving high-speed train. For LTE signals received inside the train, the average attenuation observed with the moving train is approximately $20 \sim 30 \mathrm{~dB}$ at 2.6 GHz. This large attenuation results in the cell radius with internal antennas is approximately a quarter of cell radius with external antennas. For the train parked inside the station, an example attenuation $17 \mathrm{~dB}$ is found from the station to the mobile inside the train. Hence, to provide a reliable communications systems for passengers with reasonable cell sizes, a relay system with external and internal antennas is considered to deployed. The signal inside the train carriages can be easily distributed by using WiFi access points or directly using repeaters. The radio waves propagate inside the train carriage suffer waveguiding effects due to the confined space and dense reflections from the interior surfaces. Received signal amplitudes are good fitted with Nakagami-m and Weibull distribution with $m>1$ and $\beta>2$, respectively. This implies the presence of dominant component for the Intra-Carriage, Inter-Carriage, and Station-Mobile links. The desirable frequency for signal distribution inside train carriages is $2.4 \mathrm{GHz}$ or $5.7 \mathrm{GHz}$, also considering that Inter-Carriage propagation is acceptable at these frequencies.

\subsection{Future Work}

As the flourish of $5 \mathrm{G}$ technology and internet of things (IoT), communication industry faces a revolution with big challenges, and also new opportunities. But no matter what, new wireless technologies are dedicated to further improve the performance and reliability of wireless communication systems. These systems employed new signaling schemes may operate in harsher environments at higher frequencies. Hence, some possible extensions of this dissertation work for further channel characterizations are summarized in this section.

For channel sounding system, SDR provides a promising direction, which is a universal platform with flexible configurations. Due to the lightweight design and modest resource requirements, they are also well suited for the embedded 
space and measurements in complex environments. In addition, the conventional channel sounder attached with an antenna array is helpful for MIMO testing.

For channel measurement, technical advances bring the requirements for larger and larger communication capacity, which stimulate the wireless communication system to develop towards adaptive, high speed, large capacity data transmission. Therefore, corresponding wireless channel measurements for certain goals have to be conducted with specific configurations. There are some potential directions to look forward to, as:

- Higher frequency: for the promising application with wider bandwidth, propagation models at the higher frequencies such as mmWave are still maturing. There are extensive measurements but few analytical models in railway. Possible challenges include i) very high path loss; ii) heavy oxygen absorption and rain attenuation. iii) more severe shadow fading. iv) mmWave applications are limited by the short range, massive MIMO is the possible approach to get a larger range, which leads to the requirements of further knowledge on the mmWave massive MIMO channel matrix.

- Time-variance: wideband measurements for time-varying communication channels in high mobility scenarios, such as UAV systems. Modeling the time delay spread and the "birth" and "death" processes of channel taps; Characterizing the Doppler effect and frequency dispersion in such high mobility scenarios.

- Multi-antenna system: channel measurements for MIMO system in dense multipath environments such as subway station are of interest for establishing the channel models with spatial characteristics.

- New applications: The emerging technology, such as wearable smart devices are increasingly used in medical field. Hence, channel measurements for Body Area Network (BAN) are especially helpful for accelerating new product development. Besides, the so-called device-to-device (D2D) communication between circuit boards has been an explosive growth. Moreover, The promise of Internet of Vehicles (IOV) technology is extraordinary, 
which points the channel measurements within vehicles, such as cars, ships, and UAVs are of importance.

For channel modeling, further measurement results can be applied to the highresolution multipath estimation algorithms. For instance, the classical maximumlikelihood parameter technique SAGE based on the narrowband assumption, and RiMAX framework includes dense MPCs situation. Also, the CLEAN algorithm can conduct the correlation and cancellation on the observed signal. These algorithms provide the estimation of polarimetric path weight matrix, the AoA/AoD, the time delay of arrival, and the Doppler-shift of the specular paths. Moreover, the correlation properties between the channel taps are of interest for Non-WSSUS channel. Additionally, more accurate simulators, for instance, ray tracing-based, graph-based, or hybrid approaches are expected to be refined, developed, and applied for channel model validation and improvement. 


\section{References}

[1] Wikipedia. History of information theory. https://en.wikipedia.org/ wiki/History_of_information_theory. Accessed August 20, 2016. 1

[2] David G Michelson and Saeed S Ghassemzadeh. Measurement and modeling of wireless channels. In New Directions in Wireless Communications Research, pages 1-27. Springer, 2009. 3, 16, 38, 41, 55

[3] Dewan Md Zahurul Islam, Ross Jackson, Thomas Hagen Zunder, and Arnaud Burgess. Assessing the impact of the 2011 eu transport white paper-a rail freight demand forecast up to 2050 for the eu27. European Transport Research Review, 7(3):1-9, 2015. 5

[4] E. Masson, Y. Cocheril, P. Combeau, L. Aveneau, M. Berbineau, R. Vauzelle, and E. Fayt. Radio wave propagation in curved rectangular tunnels at $5.8 \mathrm{GHz}$ for metro applications. In Pro. 11th International Conference on ITS Telecommunications (ITST), pages 81-85, St. Petersburg, Russia, 2011. 5, 61, 64

[5] Bernard Sklar. Digital Communications: Fundamentals and Applications (2nd Edition). Prentice Hall, 2 edition, 2001. 9

[6] Abhijit Mitra. Lecture notes on mobile communication. 2009. 10, 12

[7] Nathan Blaunstein and Christos Christodoulou. Radio propagation and adaptive antennas for wireless communication links: terrestrial, atmospheric and ionospheric, volume 193. John Wiley \& Sons, 2007. 12

[8] Jerry D. Gibson (Editor). Mobile Communications Handbook (Electrical Engineering Handbook). CRC Press, third edition edition, 2012. 12 
[9] Tait Communications. Channel concepts every system designer needs to understand. http://blog.taitradio.com/2015/09/23/ 9-channel-concepts-every-system-designer-needs-to-understand/. Accessed May 12, 2016. 12

[10] Junyi Li, Xinzhou Wu, and Rajiv Laroia. OFDMA mobile broadband communications: A systems approach. Cambridge University Press, 2013. 13

[11] Kaveh Pahlavan and Allen H Levesque. Wireless information networks, volume 93. John Wiley \& Sons, 2005. 14, 23

[12] Gerardo Agni Medina-Acosta, José Antonio Delgado-Penín, and Katsuyuki Haneda. An opportunistic cognitive radio communication through the exploitation of the small-scale fading mechanisms of the lte mobile channel. EURASIP Journal on Wireless Communications and Networking, 2013(1):1-16, 2013. 14

[13] Vikrant Vij. Wireless Communication. Laxmi Publications, Ltd., 2010. 14, 15

[14] Wim Vereecken and Michiel Steyaert. Ultra-Wideband Pulse-based Radio: Reliable Communication over a Wideband Channel. Springer Science \& Business Media, 2009. 15

[15] Yasamin Mostofi, Alejandro Gonzalez-Ruiz, Alireza Gaffarkhah, and Ding Li. Characterization and modeling of wireless channels for networked robotic and control systems-a comprehensive overview. In Intelligent Robots and Systems, 2009. IROS 2009. IEEE/RSJ International Conference on, pages 4849-4854. IEEE, 2009. 16

[16] MA Alim, MM Rahman, MM Hossain, and A Al Nahid. Analysis of large scale propagation models for mobile communications in urban area. arXiv preprint arXiv:1002.2187, 2010. 17

[17] Serguei Primak and Valeri Kontorovich. Wireless multi-antenna channels: modeling and simulation, volume 28. John Wiley \& Sons, 2011. 17 
[18] Sana Salous. Radio Propagation Measurement and Channel Modelling. John Wiley \& Sons, 2013. 17, 32

[19] John J Egli. Radio propagation above $40 \mathrm{mc}$ over irregular terrain. Proceedings of the IRE, 45(10):1383-1391, 1957. 18

[20] Aleksandar Neskovic, Natasa Neskovic, and George Paunovic. Modern approaches in modeling of mobile radio systems propagation environment. IEEE Communications Surveys and Tutorials, 3(3):2-12, 2000. 20

[21] Hong-Chuan Yang and Mohamed-Slim Alouini. Order statistics in wireless communications: diversity, adaptation, and scheduling in MIMO and OFDM systems. Cambridge University Press, 2011. 21

[22] Vijay Kumar Garg and Theodore S Rappaport. Wireless network evolution: 2G to 3G. Prentice Hall PTR, 2001. 23

[23] Minoru Nakagami. The m-distribution-a general formula of intensity distribution of rapid fading. Statistical Method of Radio Propagation, 1960. 23

[24] John David Parsons and Prof J David Parsons. The mobile radio propagation channel. 1992. 23

[25] Li Tang Zhu Hongbo. Analysis and simulation of nakagami fading channel with matlab. In Asia Pacific Conference on Environmental Electromagnetics, CEEM, volume 3, pages 490-494. Citeseer, 2003. 23

[26] Ke-Lin Du and Madisetti NS Swamy. Wireless communication systems: from $R F$ subsystems to $4 G$ enabling technologies. Cambridge University Press, 2010. 23

[27] Fabio Belloni. Fading models. S-88 Signal Processing Laboratory, HUT, 2004. 24

[28] Matthias Pätzold. Mobile radio channels. John Wiley \& Sons, 2011. 24 
[29] V Jeyasri Arokiamary. Mobile Communications. Technical Publications, 2009. 24, 29

[30] Tapan K Sarkar, Michael C Wicks, Magdalena Salazar-Palma, and Robert J Bonneau. Smart antennas, volume 170. John Wiley \& Sons, 2005. 24, 29

[31] Andreas F Molisch. Ultra-wide-band propagation channels. Proceedings of the IEEE, 97(2):353-371, 2009. 25, 27, 87

[32] Adel AM Saleh and Reinaldo Valenzuela. A statistical model for indoor multipath propagation. IEEE Journal on selected areas in communications, $5(2): 128-137,1987.26,44$

[33] Gonzalo Llano, Andres Navarro, and Juan C Cuellar. Frequency UWB channel. INTECH Open Access Publisher, 2011. 26, 27

[34] Andreas F Molisch, Kannan Balakrishnan, Chia-Chin Chong, Shahriar Emami, Andrew Fort, Johan Karedal, Juergen Kunisch, Hans Schantz, Ulrich Schuster, and Kai Siwiak. Ieee 802.15. 4a channel model-final report. IEEE P802, 15(04):0662, 2004. 27

[35] Liu Liu, Cheng Tao, Jiahui Qiu, Houjin Chen, Li Yu, Weihui Dong, and Yao Yuan. Position-based modeling for wireless channel on high-speed railway under a viaduct at $2.35 \mathrm{ghz}$. IEEE Journal on Selected Areas in Communications, 30(4):834-845, 2012. 28, 105, 111

[36] Jaroslaw Sadowski. Measurement of coherence bandwidth in uhf radio channels for narrowband networks. International Journal of Antennas and Propagation, 2015, 2015. 29

[37] Mathuranathan. Power delay profile. http://www.gaussianwaves.com/ 2014/07/power-delay-profile/. Accessed May 17, 2016. 29

[38] Nelson Costa and Simon Haykin. Multiple-input multiple-output channel models: theory and practice, volume 65. John Wiley \& Sons, 2010. 30, 43

[39] Harry R Anderson. Fixed broadband wireless system design. John Wiley \& Sons, 2003. 30, 32 
[40] Xiaohui Wang, Kevin Borries, Eric Anderson, and Peter Steenkiste. Network-scale emulation of general wireless channels. In Vehicular Technology Conference (VTC Fall), 2011 IEEE, pages 1-5. IEEE, 2011. 30

[41] Qiong Wu. Non-stationary vehicle-to-vehicle channel characterization. $\mathrm{PhD}$ thesis, Ohio University, 2012. 31, 32

[42] David Falconer, Sirikiat Lek Ariyavisitakul, Anader Benyamin-Seeyar, and Brian Eidson. Frequency domain equalization for single-carrier broadband wireless systems. Communications Magazine, IEEE, 40(4):58-66, 2002. 31

[43] Arvind Chandrasekaran. Intra-Vehicle Channel Characterization in the 5 GHz Band. PhD thesis, Ohio University, 2011. 32

[44] Shweta Sagari, Wade Trappe, and Larry Greenstein. Equivalent tapped delay line channel responses with reduced taps. In Vehicular Technology Conference (VTC Fall), 2013 IEEE 78th, pages 1-5. IEEE, 2013. 32

[45] Upamanyu Madhow. Fundamentals of digital communication. Cambridge University Press, 2008. 32

[46] David W Matolak. Channel modeling for vehicle-to-vehicle communications. IEEE Communications Magazine, 46(5):76-83, 2008. 32, 104

[47] Arunabha Ghosh, Jun Zhang, Jeffrey G Andrews, and Rias Muhamed. Fundamentals of LTE. Pearson Education, 2010. 32

[48] Theodore Rappaport. Wireless Communications: Principles and Practice. Prentice Hall PTR, 2001. 33, 34, 58

[49] Thomas Kurner, Dieter J. Cichon, and Werner Wiesbeck. Concepts and results for 3d digital terrain-based wave propagation models: An overview. IEEE Journal on selected areas in communications, 11(7):1002-1012, 1993. 34

[50] GARYA Thiele and T Newhouse. A hybrid technique for combining moment methods with the geometrical theory of diffraction. IEEE Transactions on Antennas and Propagation, 23(1):62-69, 1975. 34 
[51] Kane S Yee et al. Numerical solution of initial boundary value problems involving maxwells equations in isotropic media. IEEE Trans. Antennas Propag, 14(3):302-307, 1966. 34

[52] Zhengqing Yun and Magdy F Iskander. Ray tracing for radio propagation modeling: principles and applications. IEEE Access, 3:1089-1100, 2015. 34, 35

[53] Nicolai Czink, Alexis Paolo Garcia Ariza, Katsuyuki Haneda, Martin Jacob, Johan Kåredal, Martin K`aske, Jonas Medbo, Juho Poutanen, Jussi Salmi, Gerhard Steinb`ock, et al. Channel measurements. In Pervasive Mobile and Ambient Wireless Communications, pages 5-65. Springer, 2012. 38

[54] JD Parsons, DA Demery, and AMD Turkmani. Sounding techniques for wideband mobile radio channels: a review. In Communications, Speech and Vision, IEE Proceedings I, volume 138, pages 437-446. IET, 1991. 39

[55] Qi Chen. Wideband channel sounding techniques for dynamic spectrum access networks. 2009. 40, 41, 57

[56] Tom Alexander. Optimizing and Testing WLANs: Proven techniques for maximum performance. Elsevier, 2011. 40

[57] Andreas F Molisch. Wireless communications. John Wiley \& Sons, 2007. 42,44

[58] Dr Philip Nobles and Lt Richard Scott. Wideband propagation measurements onboard hms bristol. In Military Communications Conference, 2003. MILCOM'03. 2003 IEEE, volume 2, pages 1412-1415. IEEE, 2003. 43

[59] Chahé Nerguizian, Charles L Despins, Sofiène Affès, and Mourad Djadel. Radio-channel characterization of an underground mine at 2.4 ghz. Wireless Communications, IEEE Transactions on, 4(5):2441-2453, 2005. 43

[60] Dalwinder Singh, Zhen Hu, and Robert Qiu. Uwb channel sounding and channel characteristics in rectangular metal cavity. In Southeastcon, 2008. IEEE, pages 323-328. IEEE, 2008. 43 
[61] WR Young and LY Lacy. Echoes in transmission at 450 megacycles from land-to-car radio units. Proceedings of the IRE, 38(3):255-258, 1950. 43

[62] George L Turin, Fred D Clapp, Tom L Johnston, Stephen B Fine, and Dan Lavry. A statistical model of urban multipath propagation. IEEE Transactions on Vehicular Technology, 21(1):1-9, 1972. 44

[63] Jan Van Rees. Measurements of the wide-band radio channel characteristics for rural, residential, and suburban areas. IEEE Transactions on Vehicular Technology, 36(1):2-6, 1987. 44

[64] K Pahlavan, R Ganesh, and T Hotaling. Multipath propagation measurements on manufacturing floors at 910 mhz. Electronics Letters, 3(25):225227, 1989. 44

[65] Theodore S Rappaport. Characterization of uhf multipath radio channels in factory buildings. IEEE Transactions on Antennas and Propagation, 37(8):1058-1069, 1989. 44

[66] D Cox. Delay doppler characteristics of multipath propagation at $910 \mathrm{mhz}$ in a suburban mobile radio environment. IEEE Transactions on Antennas and Propagation, 20(5):625-635, 1972. 44

[67] DMJ Devasirvatham, MJ Krain, DA Rappaport, and C Banerjee. Radio propagation measurements at $850 \mathrm{mhz}, 1.7 \mathrm{ghz}$ and $4 \mathrm{ghz}$ inside two dissimilar office buildings. Electronics Letters, 26(7):445-447, 1990. 44

[68] William G Newhall, Kevin J Saldanha, and Theodore S Rappaport. Propagation time delay spread measurements at $915 \mathrm{mhz}$ in a large train yard. In Vehicular Technology Conference, 1996. Mobile Technology for the Human Race., IEEE 46th, volume 2, pages 864-868. IEEE, 1996. 44

[69] Jarmo Kivinen, Timo O Korhonen, Pauli Aikio, Ralf Gruber, Pertti Vainikainen, and S-G Haggman. Wideband radio channel measurement system at 2 ghz. IEEE transactions on Instrumentation and Measurement, 48(1):39-44, 1999. 44 
[70] JO Nielsen, Jørgen Bach Andersen, Patrick Claus F Eggers, Gert Frølund Pedersen, Kim Olesen, and H Suda. Measurements of indoor $16 \times 32$ wideband mimo channels at 5.8 ghz. In Spread Spectrum Techniques and Applications, 2004 IEEE Eighth International Symposium on, pages 864-868. IEEE, 2004. 44

[71] S Salous, P Filippidis, R Lewenz, I Hawkins, N Razavi-Ghods, and M Abdallah. Parallel receiver channel sounder for spatial and mimo characterisation of the mobile radio channel. IEE Proceedings-Communications, 152(6):912-918, 2005. 44

[72] Sylvain Ranvier, Mikko Kyr`o, Katsuyuki Haneda, Tuomas Mustonen, Clemens Icheln, and Pertti Vainikainen. Vna-based wideband 60 ghz mimo channel sounder with 3-d arrays. In Radio and Wireless Symposium, 2009. RWS'09. IEEE, pages 308-311. IEEE, 2009. 44

[73] Tobias Felhauer, Paul W Baier, Winfried K`ONIG, and MOHR Werner. Optimized wideband system for unbiased mobile radio channel sounding with periodic spread spectrum signals. IEICE Transactions on Communications, 76(8):1016-1029, 1993. 44

[74] Ralf Heddergott, Pascal Truffer, Martin Tschudin, and Michael Nold. Comparison of high resolution channel parameter measurements with ray tracing simulations in a multipath environment. In $E P M C C$, volume 99, pages 9-11. Citeseer, 1999. 44

[75] MEDAV GmbH. Rusk channel sounder. url=http://www.channelsounder.de/. Accessed June 20, 2016. 44

[76] Uwe Trautwein, Christian Schneider, Gerd Sommerkorn, Dirk Hampicke, $\mathrm{R}$ Thoma, and Walter Wirnitzer. Measurement data for propagation modeling and wireless system evaluation. COST273, MCM, TD-03-021, Barcelona, 2003. 44

[77] L Hentil`a, P Ky`osti, Juha Ylitalo, X Zhao, J Meinil’a, and JP Nuutinen. Experimental characterization of multi-dimensional parameters at 2.45 and $5.25 \mathrm{ghz}$ indoor channels. In WPMC, volume 5, pages 18-22, 2005. 44 
[78] Lei Zhang, C Briso-Rodriguez, Jean Rapha'el Olivier Fernandez, and Ke Guan. Channel sounder and broadband measurements for railway systems. In IEICE Info. Comm. Technol. Forum (ICTF), 2014. 46, 80

[79] Muhammad Nazmul Islam, Byoung-Jo J Kim, Paul Henry, and Eric Rozner. A wireless channel sounding system for rapid propagation measurements. In 2013 IEEE International Conference on Communications (ICC), pages 5720-5725. IEEE, 2013. 53

[80] Mutsawashe Gahadza, Minseok Kim, and J ichi Takada. Implementation of a channel sounder using gnu radio opensource sdr platform. The Institute of Electronics, Information and Communication Engineers (IEICE), Japan, Technical Report, 2009. 53

[81] Adrien LE NAOUR, Olivier GOUBET, Christophe MOY, and Pierre LERAY. Spread spectrum channel sounder implementation with usrp platforms. SUPELEC/IETR, Cesson-Sévigné Cedex, France, 2013. 53

[82] Leonard Novosel and Gordan Šišul. Comparison of pseudo noise sequence lengths for a correlator channel sounder. In Proceedings ELMAR-2014, pages $1-4$. IEEE, 2014. 53

[83] Hector Reyes, Naima Kaabouch, and Wen-Chen Hu. Spectrum channel characterization using delay and doppler spread parameters. Journal of Communications, 9(3), 2014. 53

[84] Xuefeng Yin, Meng Tian, Luxia Ouyang, Xiang Cheng, Xuesong Cai, Li Tian, Jiajing Chen, and Pinglu Yang. Modelling city-canyon pedestrian radio channels based on passive sounding in in-service networks. 2015. 53

[85] Xuesong Cai, Xuefeng Yin, Xiang Cheng, and Antonio Perez-Yuste. An empirical random-cluster model for subway channels based on passive measurements in umts. 2016. 53

[86] Ian F Akyildiz, Zhi Sun, and Mehmet C Vuran. Signal propagation techniques for wireless underground communication networks. Physical Communication, 2(3):167-183, 2009. 59 
[87] Steven Kisseleff, Ian F Akyildiz, and Wolfgang H Gerstacker. Digital signal transmission in magnetic induction based wireless underground sensor networks. IEEE Transactions on Communications, 63(6):2300-2311, 2015. 60

[88] Yue Ping Zhang, Guo Xin Zheng, and JH Sheng. Radio propagation at $900 \mathrm{mhz}$ in underground coal mines. IEEE transactions on antennas and propagation, 49(5):757-762, 2001. 60

[89] Suk-Un Yoon, Liang Cheng, Ehsan Ghazanfari, Sibel Pamukcu, and Muhannad T Suleiman. A radio propagation model for wireless underground sensor networks. In Global Telecommunications Conference (GLOBECOM 2011), 2011 IEEE, pages 1-5. IEEE, 2011. 60

[90] Gilbert Siy Ching, Mir Ghoraishi, Navarat Lertsirisopon, Jun-ichi Takada, Itoji Sameda, Tetsuro Imai, and Hironori Sakamoto. Wideband directional radio propagation channel analysis inside an arched tunnel. In 2006 IEEE 17th International Symposium on Personal, Indoor and Mobile Radio Communications, pages 1-5. IEEE, 2006. 60

[91] C. Briso-Rodriguez, J.M. Cruz, and J.I. Alonso. Measurements and modeling of distributed antenna systems in railway tunnels. IEEE Transactions on Vehicular Technology, 56(5):2870-2879, Sep. 2007. 60, 61

[92] K. Guan, Zh. Zhong, J. I. Alonso, and C. Briso. Measurement of distributed antenna systems at $2.4 \mathrm{GHz}$ in a realistic subway tunnel environment. IEEE Transactions on Vehicular Technology, 61(2):834-837, Feb. 2012. 60, 61, 73

[93] Martine Liénard and Pierre Degauque. Natural wave propagation in mine environments. IEEE Transactions on Antennas and Propagation, 48(9):1326-1339, 2000. 60

[94] Matti Hamalainen, Jaakko Talvitie, Veikko Hovinen, and P Leppanen. Wideband radio channel measurement in a mine. In Spread Spectrum Techniques and Applications, 1998. Proceedings., 1998 IEEE 5th International Symposium on, volume 2, pages 522-526. IEEE, 1998. 60 
[95] Ahmed Benzakour, Sofiene Affes, Charles Despins, and P-M Tardif. Wideband measurements of channel characteristics at 2.4 and 5.8 ghz in underground mining environments. In Vehicular Technology Conference, 2004. VTC2004-Fall. 2004 IEEE 60th, volume 5, pages 3595-3599. IEEE, 2004. 60

[96] Abdellah Chehri, Paul Fortier, and Pierre Martin Tardif. Characterization of the ultra-wideband channel in confined environments with diffracting rough surfaces. Wireless Personal Communications, 62(4):859-877, 2012. 60

[97] K. Dar, M. Bakhouya, J. Gaber, M. Wack, and P. Lorenz. Wireless communication technologies for ITS applications [topics in automotive networking]. IEEE Communications Magazine, 48(5):156-162, May 2010. 60

[98] H. Wang, F.R. Yu, L. Zhu, T. Tang, and B. Ning. Finite-state Markov modeling for wireless channels in tunnel communication-based train control systems. IEEE Transactions on Intelligent Transportation Systems, 15(3):1083-1090, Jun. 2014. 60

[99] X. Cheng, X. Hu, L. Yang, I. Husain, K. Inoue, P. Krein, R. Lefevre, Y. Li, H. Nishi, J. Taiber, F. Wang, Y. Cha, W. Gao, and Z. Li. Electrified vehicles and the smart grid: the its perspective. IEEE Transactions on Intelligent Transportation Systems, 15(4):1388-1404, Aug. 2014. 60

[100] X. Cheng, C.-X. Wang, H. Wang, X. Gao, X.-H. You, D. Yuan, B. Ai, Q. Huo, L. Song, and B. Jiao. Cooperative mimo channel modeling and multi-link spatial correlation properties. IEEE Journal on Selected Areas in Communications, 30(2):388-396, Feb. 2012. 60

[101] D.G. Dudley, M. Lienard, S.F. Mahmoud, and P. Degauque. Wireless propagation in tunnels. IEEE Antennas and Propagation Magazine, 49(2):11-26, April 2007. 61

[102] A. Hrovat, G. Kandus, and T. Javornik. Four-slope channel model for path loss prediction in tunnels at $400 \mathrm{MHz}$. IET Microwaves, Antennas and Propagation, 4(5):571-582, May 2010. 61 
[103] X. Cheng, C.-X. Wang, D. Laurenson, S. Salous, and A. Vasilakos. An adaptive geometry-based stochastic model for non-isotropic mimo mobileto-mobile channels. IEEE Trans. Wireless Comm., 8(9):4824-4835, Sept. 2009. 61

[104] Y. Zhang and Y. Hwang. Characterization of UHF radio propagation channels in tunnel environments for microcellular and personal communications. IEEE Transactions on Vehicular Technology, 47(1):283-296, Feb. 1998. 61

[105] Y. Zhang. Novel model for propagation loss prediction in tunnels. IEEE Transactions on Vehicular Technology, 52(5):1308-1314, Sep. 2003. 61

[106] Ke Guan, Zhangdui Zhong, Bo Ai, Ruisi He, Yuanxuan Li, and Cesar Briso. Propagation mechanism modeling in the near-region of arbitrary cross-sectional tunnels. International Journal of Antennas and Propagation, 2012:11, 2012. 61

[107] A. Hrovat and T. Javornik. Analysis of radio propagation models for smart city applications. International Journal of Communications, 7(4):83-92, 2013. 61

[108] K. Guan, Z. Zhong, B. Ai, R. He, B. Chen, Y. Li, and C. Briso. Complete propagation model in tunnels. IEEE Antennas and Wireless Propagation Letters, 12:741-744, Jul. 2013. 61

[109] K. Guan, Z. Zhong, B. Ai, R. S. He, and C. Briso. Five-zone propagation model for large-size vehicles inside tunnels. Progress In Electromagnetics Research, 138:389-405, Jul. 2013. 61

[110] K. Guan, Z. Zhong, B. Ai, R. S. He, B. H. Chen, Y. X. Li, and C. Briso. Complete propagation model structure inside tunnels. Progress In Electromagnetics Research, 141:711-726, Aug. 2013. 61

[111] K. Guan, Zh. Zhong, B. Ai, and C. Briso. Propagation mechanism analysis before the break point inside tunnels. In IEEE 74th Veh. Technol. Conf., pages 1-5, San Francisco, CA, USA, 2011. 61 
[112] D. Didascalou, T.M. Schafer, F. Weinmann, and W. Wiesbeck. Raydensity normalization for ray-optical wave propagation modeling in arbitrarily shaped tunnels. IEEE Transactions on Antennas and Propagation, 48(9):1316-1325, Sep. 2000. 61

[113] F.M. Pallares, F.J.P. Juan, and L. Juan-Llacer. Analysis of path loss and delay spread at $900 \mathrm{MHz}$ and $2.1 \mathrm{GHz}$ while entering tunnels. IEEE Transactions on Vehicular Technology, 50(3):767-776, May 2001. 61

[114] Alexei V. Popov and Ning Yan Zhu. Modeling radio wave propagation in tunnels with a vectorial parabolic equation. IEEE Transactions on Antennas and Propagation, 48(9):1403-1412, Sep. 2000. 61

[115] Y. Zhang, Y. Hwang, and P. Ching. Characterization of UHF radio propagation channel in curved tunnels. In Proc. Seventh IEEE International Symposium on Personal, Indoor and Mobile Radio Communications, PIMRC'96., volume 3, pages 798-802, Taipei, 1996. 61, 71

[116] D. Didascalou, M. Dottling, T. Zwick, and W. Wiesbeck. A novel rayoptical approach to model wave propagation in curved tunnels. In IEEE VTS 50th Vehicular Technology Conference, volume 4, pages 2313-2317, Amsterdam, the Netherlands, 1999. 61

[117] J. S. Lamminmaki and J. J. A. Lempiainen. Radio propagation characteristics in curved tunnels. IEE Proceedings Microwaves, Antennas and Propagation, 145(4):327-331, Aug. 1998. 61

[118] M. Lienard, S. Betrencourt, and P. Degauque. Theoretical and experimental approach of the propagation at $2.5 \mathrm{GHz}$ and $10 \mathrm{GHz}$ in straight and curved tunnels. In Vehicular Technology Conference, 1999. VTC 1999 - Fall. IEEE VTS 50th, volume 4, pages 2268-2271 vol.4. 61, 64

[119] X. Cheng, Q. Yao, C.-X. Wang, B. Ai, G. L. Stuber, D. Yuan, and B. Jiao. An improved parameter computation method for a mimo v2v rayleigh fading channel simulator under non-isotropic scattering environments. IEEE Commun. Letters, 17(2):265-268, Feb. 2013. 61 
[120] N. Sood, Liang Liang, S. V. Hum, and C. D. Sarris. Ray-tracing based modeling of ultra-wideband pulse propagation in railway tunnels. In Proc. 2011 IEEE International Symposium on Antennas and Propagation (APSURSI), pages 2383-2386, Spokane, WA, USA, 2011. 61

[121] Wang Te-Shun and Yang Chang-Fa. Simulations and measurements of wave propagations in curved road tunnels for signals from GSM base stations. IEEE Transactions on Antennas and Propagation, 54(9):2577-2584, Sep. 2006. 61,64

[122] X. Cheng, C.-X. Wang, D. Laurenson, S. Salous, and A. Vasilakos. New deterministic and stochastic simulation models for non-isotropic scattering mobile-to-mobile rayleigh fading channels. Wireless Communications and Mobile Computing, John Wiley \& Sons, 11(7):829-842, Jul. 2011. 61

[123] R. Martelly and R. Janaswamy. Modeling radio transmission loss in curved, branched and rough-walled tunnels with the ADI-PE method. IEEE Transactions on Antennas and Propagation, 58(6):2037-2045, 2010. 61, 64

[124] P. Bernardi, D. Caratelli, R. Cicchetti, V. Schena, and O. Testa. A numerical scheme for the solution of the vector parabolic equation governing the radio wave propagation in straight and curved rectangular tunnels. IEEE Transactions on Antennas and Propagation, 57(10):3249-3257, 2009. 61

[125] M. Nilsson, J. Slettenmark, and C. Beckman. Wave propagation in curved road tunnels. In Antennas and Propagation Society International Symposium, 1998. IEEE, volume 4, pages 1876-1879 vol.4. 61

[126] S. F. Mahmoud. On modal propagation of high frequency electromagnetic waves in straight and curved tunnels. In Antennas and Propagation Society International Symposium, 2004. IEEE, volume 3, pages 2963-2966 Vol.3. 61

[127] W. C Y Lee. Estimate of local average power of a mobile radio signal. IEEE Transactions on Vehicular Technology, 34(1):22-27, Feb. 1985. 64 
[128] M. Gudmundson. Correlation model for shadow fading in mobile radio systems. Electronics Letters, 27, Nov. 1991. 67, 68

[129] Guidelines for evaluation of radio transmission technologies for IMT-2000. (Rec.ITU-R M.1225), 1997. 67

[130] WINNER II channel models. (Deliverable 1.1.2 v.1.2), 2007. 67, 72, 74

[131] 3rd generation partnership project; technical specification group radio access networks; spatial channel model for MIMO simulations (release 6). (V6.1.0). 68

[132] Multi-hop relay systemevaluation methodology (channel model and performance metrics). (IEEE802.16j-06/013r3), 2007. 68

[133] Guidelines for evaluation of radio interface technologies for IMT-Advanced. (Tech. Rep. ITU-R M.2135), 2008. 68

[134] S.S. Szyszkowicz, H. Yanikomeroglu, and J.S. Thompson. On the feasibility of wireless shadowing correlation models. IEEE Transactions on Vehicular Technology, 59(9):4222-4236, Nov. 2010. 68

[135] Jiping Sun and Chuanlei Zhang. Analysis of transmission characteristics of curved tunnel with arbitrary cross section using hybrid mode matching/finite elements approach. In Proc. IEEE International Symposium on Communications and Information Technology, volume 1, pages 63-66, 2005. 71

[136] Ningde Municipal Science and Technology Bureau. Ningde high-speed railway tunnel covering research report. Technical report, 2008. 73

[137] J. M. Molina-Garcia-Pardo, M. Lienard, and P. Degauque. Propagation in tunnels: Experimental investigations and channel modeling in a wide frequency band for MIMO applications. EURASIP Journal on Wireless Communications and Networking, 2009, 2009. 73

[138] Nan Wang. A uniform path-loss model by power balance theory (PBT) and its application on tunnels. In Antennas Propagation and EM Theory 
(ISAPE), 2010 9th International Symposium on, pages 481-484, Nov 2010. 73

[139] D. Didascalou, J. Maurer, and W. Wiesbeck. Subway tunnel guided electromagnetic wave propagation at mobile communications frequencies. IEEE Transactions on Antennas and Propagation, 49(11):1590-1596, Nov 2001. 73

[140] S. Shinozaki, M. Wada, A Teranishi, H. Furukawa, and Y. Akaiwa. Radio propagation characteristics in subway platform and tunnel in $2.5 \mathrm{ghz}$ band. In Sixth IEEE International Symposium on Personal, Indoor and Mobile Radio Communications, 1995. PIMRC'95. Wireless: Merging onto the Information Superhighway, volume 3, pages 1175-, Sept 1995. 73

[141] Myung-Sun Choi, Do-Youn Kim, Han-Shin Jo, Jong-Gwan Yook, and HanKyu Park. Path-loss characteristics in subway tunnels at 2.65 ghz. Microwave and Optical Technology Letters, 48(2):383-386, 2006. 73

[142] K. Guan, Z. Zhong, B. Ai, and T. Kuerner. Propagation measurements and analysis for train stations of high-speed railway at $930 \mathrm{MHz}$. IEEE Transactions on Vehicular Technology, 63(8):3499-3516, 2014. 73

[143] K. Guan, Z. Zhong, B. Ai, and T. Kuerner. Empirical models for extra propagation loss of train stations on high-speed railway. IEEE Transactions on Antennas and Propagation, 62(3):1395-1408, Mar. 2014. 73

[144] K. Guan, Z. Zhong, B. Ai, and T. Kuerner. Propagation measurements and modeling of crossing bridges on high-speed railway at $930 \mathrm{MHz}$. IEEE Transactions on Vehicular Technology, 63(2):502-517, Feb. 2014. 73

[145] K. Guan, Z. Zhong, B. Ai, and T. Kuerner. Propagation prediction for composite scenarios of dense semi-closed obstacles in high-speed railway. In 31th URSI General Assembly and Scientific Symposium (31th URSI GASS), pages 1-4, Aug. 2014. 73 
[146] R. He, Z. Zhong, B. Ai, and J. Ding. Propagation measurements and analysis for high-speed railway cutting scenario. Electronics Letters, 47(21):11671168, Oct. 2011. 73

[147] Ruisi He, Zhangdui Zhong, Bo Ai, and Jianwen Ding. Measurements and analysis of short-term fading behavior for high-speed rail viaduct scenario. In Communications (ICC), 2012 IEEE International Conference on, pages 4563-4567, Jun. 2012. 73

[148] Liu Liu, Cheng Tao, Jiahui Qiu, Houjin Chen, Li Yu, Weihui Dong, and Yao Yuan. Position-based modeling for wireless channel on high-speed railway under a viaduct at $2.35 \mathrm{GHz}$. IEEE Journal on Selected Areas in Communications, 30(4):834-845, May 2012. 73

[149] Y. Zhang, J. Zhang, D. Dong, X. Nie, G. Liu, and P. Zhang. A novel spatial autocorrelation model of shadow fading in urban macro environments. In Proc. IEEE Global Telecommunications Conference, pages 1-5, New Orleans, LO, USA, Nov. 2008. 74

[150] Fengyu Luan, Yan Zhang, Limin Xiao, Chunhui Zhou, and Shidong Zhou. Fading characteristics of wireless channel on high-speed railway in hilly terrain scenario. International Journal of Antennas and Propagation, 2013:9, 2013. 74

[151] Hong Wei, Zhangdui Zhong, Lei Xiong, Bo Ai, and Ruisi He. Study on the shadow fading characteristic in viaduct scenario of the high-speed railway. In Proc. 6th International ICST Conference on Communications and Networking in China (CHINACOM), pages 1216-1220, Harbin, China, Aug. 2011. 74

[152] Andreas F Molisch. Statistical properties of the rms delay-spread of mobile radio channels with independent rayleigh-fading paths. IEEE transactions on vehicular technology, 45(1):201-204, 1996. 84

[153] Hristo Karagiozov. Lecture 6 reverberation. http://karagioza.com/?p= 676. Accessed May 24, 2016. 86 
[154] J Bach Andersen, J Ø Nielsen, GF Pedersen, G Bauch, and M Herdin. Room electromagnetics. Antennas and Propagation Magazine, IEEE, 49(2):27-33, 2007. 87, 88, 89

[155] Gerhard Steinbock, Troels Pedersen, Bernard H Fleury, Wei Wang, Thomas Jost, and Ronald Raulefs. Model for the path loss of in-room reverberant channels. In Vehicular Technology Conference (VTC Spring), 2011 IEEE 73rd, pages 1-5. IEEE, 2011. 87

[156] Gerhard Steinbock, Troels Pedersen, Bernard Henri Fleury, Wei Wang, and Ronald Raulefs. Experimental validation of the reverberation effect in room electromagnetics. Antennas and Propagation, IEEE Transactions on, 63(5):2041-2053, 2015. 88, 89

[157] Carl F Eyring. Reverberation time in dead rooms. The Journal of the Acoustical Society of America, 1(2A):168-168, 1930. 90

[158] Aliou Bamba, Wout Joseph, Jørgen Bach Andersen, Emmeric Tanghe, Günter Vermeeren, David Plets, Jesper Ødum Nielsen, and Luc Martens. Experimental assessment of specific absorption rate using room electromagnetics. IEEE Transactions on Electromagnetic Compatibility, 54(4):747757, 2012. 90

[159] Aliou Bamba, Maria-Teresa Martinez-Ingles, Davy P Gaillot, Emmeric Tanghe, Brecht Hanssens, Jose-Maria Molina-Garcia-Pardo, Martine Lienard, Luc Martens, and Wout Joseph. Experimental investigation of electromagnetic reverberation characteristics as a function of uwb frequencies. IEEE Antennas and Wireless Propagation Letters, 14:859-862, 2015. 90, 95

[160] Mohsen KavehradZhou ZhouM. I. Sakib Chowdhury. Short Range Optical Wireless: Theory and Applications. John Wiley \& Sons, United Kingdom, 2016. 92

[161] Su-Khiong Yong, Pengfei Xia, and Alberto Valdes-Garcia. 60GHz Technology for Gbps WLAN and WPAN: from Theory to Practice. John Wiley \& Sons, 2011. 92 
[162] Ke Guan, Zhangdui Zhong, Bo Ai, Ruisi He, Binghao Chen, Yuanxuan Li, and Cesar Briso-Rodriguez. Complete propagation model in tunnels. IEEE Antennas and Wireless Propagation Letters, 12:741-744, 2013. 94

[163] David A Hill. Electromagnetic fields in cavities: deterministic and statistical theories, volume 35. John Wiley \& Sons, 2009. 95

[164] Vegnesh Rajamani, Charles F Bunting, and James C West. Differences in quality factor estimation in frequency and time domain. In 2012 AsiaPacific Symposium on Electromagnetic Compatibility, pages 505-508. IEEE, 2012. 95

[165] Jingxian Wu and Pingzhi Fan. A survey on high mobility wireless communications: Challenges, opportunities and solutions. IEEE Access, 4:450-476, 2016. 103,128

[166] Bo Ai, Xiang Cheng, Thomas K’urner, Zhang-Dui Zhong, Ke Guan, Rui-Si He, Lei Xiong, David W Matolak, David G Michelson, and Cesar BrisoRodriguez. Challenges toward wireless communications for high-speed railway. IEEE transactions on intelligent transportation systems, 15(5):21432158, 2014. 103

[167] Qiong Wu, David W Matolak, and Indranil Sen. 5-ghz-band vehicle-tovehicle channels: models for multiple values of channel bandwidth. IEEE Transactions on Vehicular Technology, 59(5):2620-2625, 2010. 104

[168] Mate Boban, Joao Barros, and Ozan K Tonguz. Geometry-based vehicleto-vehicle channel modeling for large-scale simulation. IEEE Transactions on Vehicular Technology, 63(9):4146-4164, 2014. 104

[169] David W Matolak. Air-ground channels \& models: Comprehensive review and considerations for unmanned aircraft systems. In Aerospace Conference, 2012 IEEE, pages 1-17. IEEE, 2012. 104

[170] Evşen Yanmaz, Robert Kuschnig, and Christian Bettstetter. Channel measurements over 802.11 a-based uav-to-ground links. In 2011 IEEE GLOBECOM Workshops (GC Wkshps), pages 1280-1284. IEEE, 2011. 104 
[171] David W Matolak and Ruoyu Sun. Ag channel measurement and modeling results for over-water and hilly terrain conditions. 2015. 104

[172] Ruisi He, Zhangdui Zhong, Bo Ai, and Jianwen Ding. An empirical path loss model and fading analysis for high-speed railway viaduct scenarios. Antennas and Wireless Propagation Letters IEEE, 10(10):808-812, 2011. 105

[173] Ruisi He, Zhangdui Zhong, Bo Ai, Gongpu Wang, Jianwen Ding, and A. F. Molisch. Measurements and analysis of propagation channels in highspeed railway viaducts. IEEE Transactions on Wireless Communications, 12(2):794-805, 2013. 105

[174] Fengyu Luan, Yan Zhang, Limin Xiao, Chunhui Zhou, and Shidong Zhou. Fading characteristics of wireless channel on high-speed railway in hilly terrain scenario. International Journal of Antennas and Propagation, 2013, 2013. 105, 111

[175] Yan Zhang, Zunwen He, Wancheng Zhang, Limin Xiao, and Shidong Zhou. Measurement-based delay and doppler characterizations for high-speed railway hilly scenario. International Journal of Antennas and Propagation, 2014, 2014. 105, 111

[176] R. He, Z. Zhong, B. Ai, and J. Ding. Propagation measurements and analysis for high-speed railway cutting scenario. Electronics Letters, 47(21):1167 - 1168, 2011. 105

[177] Ruisi He, Zhangdui Zhong, Bo Ai, Jianwen Ding, Yaoqing Yang, and A. F. Molisch. Short-term fading behavior in high-speed railway cutting scenario: Measurements, analysis, and statistical models. IEEE Transactions on Antennas and Propagation, 61(4):2209-2222, 2013. 105

[178] Binghao Chen, Zhangdui Zhong, Bo Ai, and David G. Michelson. A geometry-based stochastic channel model for high-speed railway cutting scenarios. IEEE Antennas and Wireless Propagation Letters, 14:851-854, 2015. 105 
[179] Ke Guan, Zhangdui Zhong, Bo Ai, and Thomas Kurner. Propagation measurements and analysis for train stations of high-speed railway at $930 \mathrm{mhz}$. IEEE Transactions on Vehicular Technology, 63(8):3499-3516, 2014. 105, 107

[180] Ke Guan, Zhangdui Zhong, and Bo Ai. Empirical models for extra propagation loss of train stations on high-speed railway. IEEE Transactions on Antennas and Propagation, 62(3):1395-1408, 2014. 105, 107

[181] Cesar Briso-Rodrguez, Javier M. Cruz, and Jose /I/. Alonso. Measurements and modeling of distributed antenna systems in railway tunnels. IEEE Transactions on Vehicular Technology, 56(5):2870-2879, 2007. 105

[182] J. Alonso, S. Capdevila, B. Izquierdo, and J. Romeu. Propagation measurements and simulations in tunnel environment at $5.8 \mathrm{ghz}$. In Antennas and Propagation Society International Symposium, IEEE, pages 1-4, 2008. 105

[183] Qian Wang, Chunxiu Xu, Min Zhao, and Siyu Zhang. Measurement-based tapped-delay-line (tdl) models for wireless channels under high speed railway scenarios at $2.6 \mathrm{ghz}$. In 21st International Conference on Telecommunications (ICT), pages 353-357, May 2014. 105

[184] Yaoqing Yang and Pingyi Fan. Doppler frequency offset estimation and diversity reception scheme of high-speed railway with multiple antennas on separated carriage. Journal of Modern Transportation, 20(4):227-233, 2012. 105

[185] Shi Pu, Junhong Wang, and Zheng Li. Integrative modeling and analyses of the wireless link for communication system in railway environment. International Symposium on Antennas Propagation \& Em Theory, pages 1322-1325, 2008. 105

[186] Ke Guan, Zhangdui Zhong, Bo Ai, and T. Kürner. Deterministic propagation modeling for the realistic high-speed railway environment. In 2013 IEEE 7rth Vehicular Technology Conference (VTC Spring), pages 1 - 5, 2013. 105,117 
[187] Pengyu Liu, Bo Ai, David Matolak, Ruoyu Sun, and Yan Li. Shadow fading analysis for high-speed railway station channels. 107

[188] Tao Zhou, Cheng Tao, Liu Liu, Jiahui Qiu, and Rongchen Sun. High-speed railway channel measurements and characterizations: a review. Journal of Modern Transportation, 20(4):199-205, 2012. 107

[189] Indranil Sen and David W Matolak. Vehicle-vehicle channel models for the 5-ghz band. IEEE Transactions on Intelligent Transportation Systems, 9(2):235-245, 2008. 116

[190] Franz Hlawatsch Gerald Matz. Wireless Communications over Rapidly Time-Varying Channels. Academic Press, 2011. 119

[191] A. Ghazal, Cheng-Xiang Wang, Bo Ai, Dongfeng Yuan, and H. Haas. A nonstationary wideband mimo channel model for high-mobility intelligent transportation systems. IEEE Transactions on Intelligent Transportation Systems, 16(2):885-897, April 2015. 119

[192] Chen Binghao, Zhangdui Zhong, and Bo Ai. Stationarity intervals of timevariant channel in high speed railway scenario. China Communications, 9(8):64-70, 2012. 119

[193] www.powertruck.com. Communications for the transport sector. http://www.powertrunk. com/wp-content/uploads/2014/02/ transport_en.pdf. Accessed August 16, 2016. 127

[194] Alain Bertout and E Bernard. Next generation of railways and metros wireless communication systems. ASPECT, Institution of Railways Signal Engineers, 2012. 127

[195] 3GPP. Technical specification group radio access network; mobile relay for E-UTRA. Technical Report 3GPP TR 36.836, 2012. 129

[196] César Briso-Rodríguez, Carlos F. López, Jean R. Fernández, Sergio Pérez, Drasko Draskovic, Jaime Calle-Sánchez, Mariano Molina, José I. Alomso, Carlos Rodríguez, Carlos Hernández, Juan Moreno, José Rodríguez-Piñeiro, 
José A. García-Naya, and Luis Castedo. Broadband Access in Complex Environment: LTE on Railway. IEICE Transactions on Communications, 97(8):1514-1527, 2014. Special Section on EU's FP7 R\&D Project Activities on Future Broadband Access Technologies. Online access: http://dx.doi.org/10.1587/transcom.E97.B.1514. 129

[197] Keith Chetcuti, Carl J Debono, Reuben A Farrugia, and Serge Bruillot. Wireless propagation modelling inside a business jet. In EUROCON 2009, EUROCON'09. IEEE, pages 1644-1649. IEEE, 2009. 129

[198] Nektarios Moraitis and Philip Constantinou. Radio channel measurements and characterization inside aircrafts for in-cabin wireless networks. In $\mathrm{Ve}$ hicular Technology Conference, 2008. VTC 2008-Fall. IEEE 68th, pages 1-5. IEEE, 2008. 129, 147

[199] Christos G Spiliotopoulos and Athanasios G Kanatas. Path-loss and timedispersion parameters of uwb signals in a military airplane. IEEE Antennas and Wireless Propagation Letters, 8:790-793, 2009. 129, 147

[200] Raffaele D'Errico and Lionel Rudant. Uhf radio channel characterization for wireless sensor networks within an aircraft. In Proceedings of the 5th European Conference on Antennas and Propagation (EUCAP), 2011. 129

[201] N Riera Diaz and Juan E Jiménez Esquitino. Wideband channel characterization for wireless communications inside a short haul aircraft. In $V e$ hicular Technology Conference, 2004. VTC 2004-Spring. 2004 IEEE 59th, volume 1, pages 223-228. IEEE, 2004. 129

[202] David W Matolak and Arvind Chandrasekaran. Aircraft intra-vehicular channel characterization in the $5 \mathrm{ghz}$ band. In 2008 Integrated Communications, Navigation and Surveillance Conference, pages 1-6. IEEE, 2008. 129

[203] Martin Jacob, Kin Lien Chee, Ingo Schmidt, Jens Schuur, Wolfgang Fischer, Martin Schirrmacher, and Thomas Kurner. Influence of passengers on the uwb propagation channel within a large wide-bodied aircraft. In 2009 3rd 
European Conference on Antennas and Propagation, pages 882-886. IEEE, 2009. 129

[204] Simon Chiu, James Chuang, and David G Michelson. Characterization of uwb channel impulse responses within the passenger cabin of a boeing 737200 aircraft. IEEE Transactions on antennas and Propagation, 58(3):935945, 2010. 129

[205] David W. Matolak and Arvind Chandrasekaran. 5 ghz intra-vehicle channel characterization. In Vehicular Technology Conference (VTC Fall), 2012 IEEE, pages 1-5. IEEE, 2012. 129

[206] David W. Matolak and Ruoyu Sun. Channel characteristics for elevator shafts at $5 \mathrm{GHz}$. In Proc. of IEEE Global Communications Conference (GLOBECOM 2013), pages 3931-3935. IEEE, 2013. 129

[207] Fredrik Harrysson, Jonas Medbo, Torbjorn Hult, and Fredrik Tufvesson. Experimental investigation of the directional outdoor-to-in-car propagation channel. IEEE Transactions on Vehicular Technology, 62(6):2532-2543, 2013. 129

[208] Pedro Suárez-Casal, José Rodríguez-Piñeiro, José A. García-Naya, and Luis Castedo. Experimental evaluation of the WiMAX downlink physical layer in high-mobility scenarios. EURASIP Journal on Wireless Communications and Networking, 2015(109), Decemeber 2014. Special Issue on Experimental Evaluation in Wireless Communications. Online access: http://dx.doi. org/10.1186/s13638-015-0339-9. 129

[209] J Meinilä, P Kyösti, L Hentilä, T Jämsä, E Suikkanen, E Kunnari, and M Narandzic. D5. 3: Winner+ final channel models. Wireless World Initiative New Radio WINNER, 2010. 129, 147

[210] Myung-Sun Choi, Do-Youn Kim, Han-Shin Jo, Jong-Gwan Yook, and HanKyu Park. Path-loss characteristics in subway tunnels at 2.65 ghz. Microwave and optical technology letters, 48(2):383-386, 2006. 129, 147 
[211] K. Guan, B. Ai, Z. Zhong, C. F. López, L. Zhang, C. Briso-Rodríguez, A. Hrovat, B. Zhang, R. He, and T. Tang. Measurements and analysis of large-scale fading characteristics in curved subway tunnels at $920 \mathrm{MHz}$, $2400 \mathrm{MHz}$, and $5705 \mathrm{MHz}$. IEEE Transactions on Intelligent Transportation Systems, 16(5):2393-2405, Oct 2015. 129, 141, 146, 147

[212] Lei Zhang, Pedro Suárez-Casal, Jean Fernández, José RodríguezPiñeiro, Jaime Calle-Sánchez, José Antonio García-Naya, Luis Castedo, C. Rodríguez-Sánchez, J. Moreno, , César Briso-Rodríguez, and J. I. Alonso-Montes. Experimental evaluation of $4 \mathrm{G}$ technologies in metro tunnel scenarios. In 10th European Conference on Antennas and Propagation (EuCAP 2016), Davos, Switzerland, April 2016. Accepted for publication. 129

[213] N. Kita, T. Ito, S. Yokoyama, M. C. Tseng, Y. Sagawa, M. Ogasawara, and M. Nakatsugawa. Experimental study of propagation characteristics for wireless communications in high-speed train cars. In 2009 3rd European Conference on Antennas and Propagation, pages 897-901, March 2009. 129, $142,145,147$

[214] Andrea Mariscotti, Attilio Marrese, Nicola Pasquino, and Rosario Schiano Lo Moriello. Characterization of the radio propagation channel aboard trains for optimal coverage at $2.45 \mathrm{GHz}$. In Proc. of IEEE International Workshop on Measurements and Networking Proceedings (MESN 2013), pages 195-199. IEEE, 2013. 129, 145, 147

[215] Weihui Dong, Guangyi Liu, Li Yu, Haiyu Ding, and Lianhua Zhang. Channel properties of indoor part for high-speed train based on wideband channel measurement. In Proc. of 5th International ICST Conference on Communications and Networking in China (CHINACOM 2010), pages 1-4. IEEE, 2010. 129

[216] Masami Shirafune, Takashi Hikage, Toshio Nojima, Motoharu Sasaki, Wataru Yamada, and Takatoshi Sugiyama. Propagation characteristic estimations of $2 \mathrm{GHz}$ inter-car wireless links in high-speed train cars in a railway 
tunnel. In Proc. of International Symposium on Antennas and Propagation (ISAP 2014), pages 215-216. IEEE, 2014. 129

[217] Toshio Ito, Naoki Kita, Wataru Yamada, Ming-Chien Tseng, Yuichi Sagawa, Mamoru Ogasawara, Masashi Nakatsugawa, and Takatoshi Sugiyama. Study of propagation model and fading characteristics for wireless relay system between long-haul train cars. In Proc. of the 5th European Conference on Antennas and Propagation (EUCAP 2011), pages 2047-2051. IEEE, 2011. 129, 148

[218] José Rodríguez-Piñeiro, Martin Lerch, José A. García-Naya, Sebastian Caban, Markus Rupp, and Luis Castedo. Emulating extreme velocities of mobile LTE receivers in the downlink. EURASIP Journal on Wireless Communications and Networking, 2015(106), April 2015. Special Issue on Experimental Evaluation in Wireless Communications. Online access: http://dx.doi.org/10.1186/s13638-015-0343-0. 129

[219] José Rodríguez-Piñeiro, Martin Lerch, Pedro Suárez-Casal, José A. GarcíaNaya, Sebastian Caban, Markus Rupp, and Luis Castedo. LTE downlink performance in high speed trains. In 2015 IEEE 81st Vehicular Technology Conference (VTC2015-Spring), Glasgow, United Kingdom, May 2015. 129

[220] José Rodríguez-Piñeiro, José A. García-Naya, Ángel Carro-Lagoa, and Luis Castedo. A Testbed for Evaluating LTE in High-Speed Trains. In Proc. of 16th Euromicro Conference on Digital System Design (DSD 2013), pages 175-182, September 2013. Online access: http://dx.doi.org/10.1109/ DSD.2013.27. 134

[221] R. He, Z. Zhong, B. Ai, J. Ding, Y. Yang, and A. F. Molisch. Shortterm fading behavior in high-speed railway cutting scenario: Measurements, analysis, and statistical models. IEEE Transactions on Antennas and Propagation, 61(4):2209-2222, April 2013. 146

[222] Eric-Jan Wagenmakers and Simon Farrell. Aic model selection using akaike weights. Psychonomic bulletin \& review, 11(1):192-196, 2004. 146 
[223] Hirofumi Suzuki. A statistical model for urban radio propogation. IEEE Transactions on Communications, 25(7):673-680, 1977. 148

[224] I. Sen, D. W. Matolak, and W. Xiong. Wireless channels that exhibit "worse than rayleigh" fading: Analytical and measurement results. In MILCOM 2006 - 2006 IEEE Military Communications conference, pages 1-7, Oct 2006. 148

[225] P Kyösti, J Meinilä, L Hentilä, X Zhao, T Jämsä, C Schneider, M Narandžić, M Milojević, A Hong, J Ylitalo, et al. Ist-4-027756 winner ii deliverable 1.1. 2. v. 1.2, winner ii channel models. IST-WINNER2, Tech. Rep, 2007. 149 


\section{Appendix: Antenna Patterns}

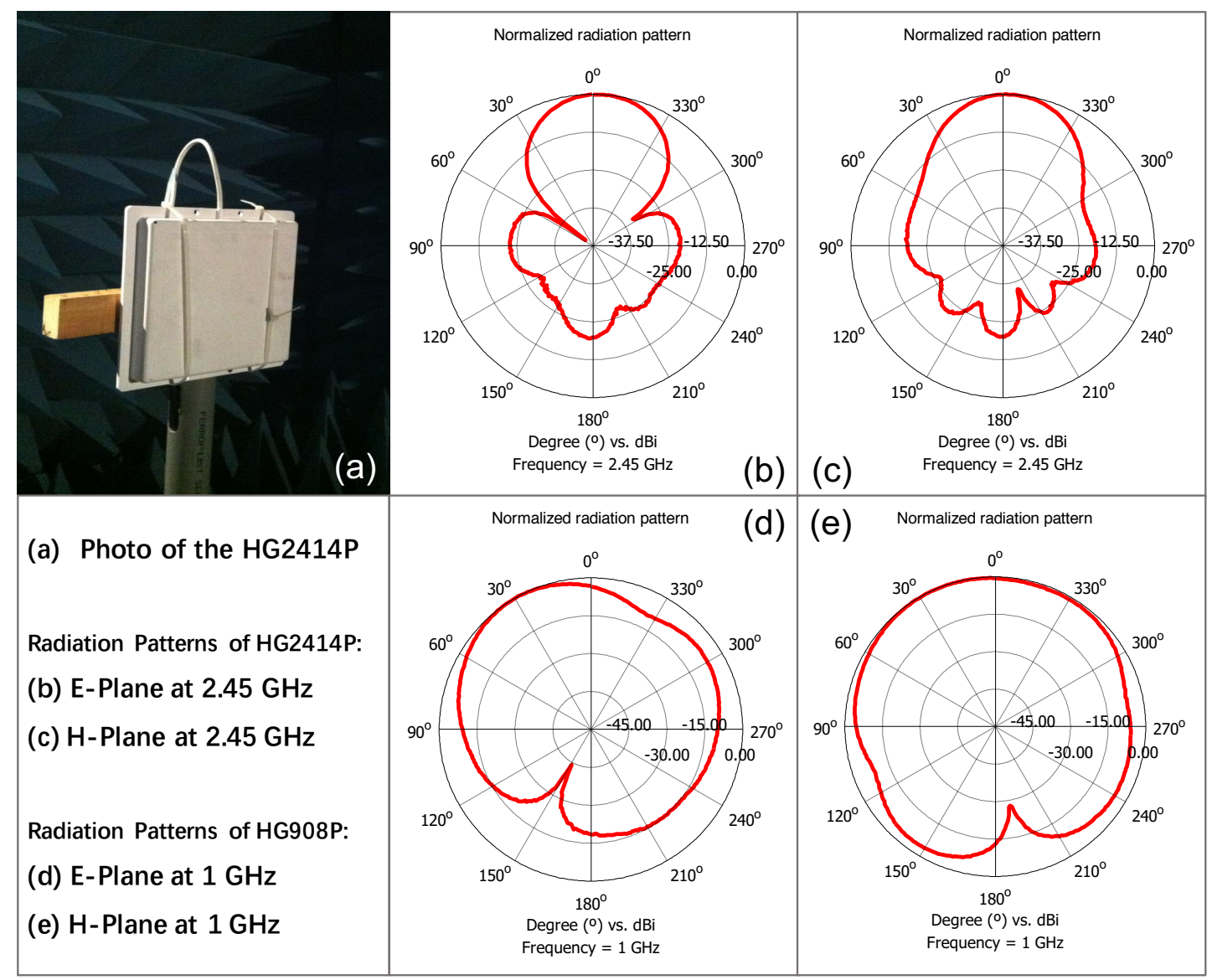

Figure 1. The photo of the HG2414P flat panel antennas and the radiation patterns at two frequencies. 


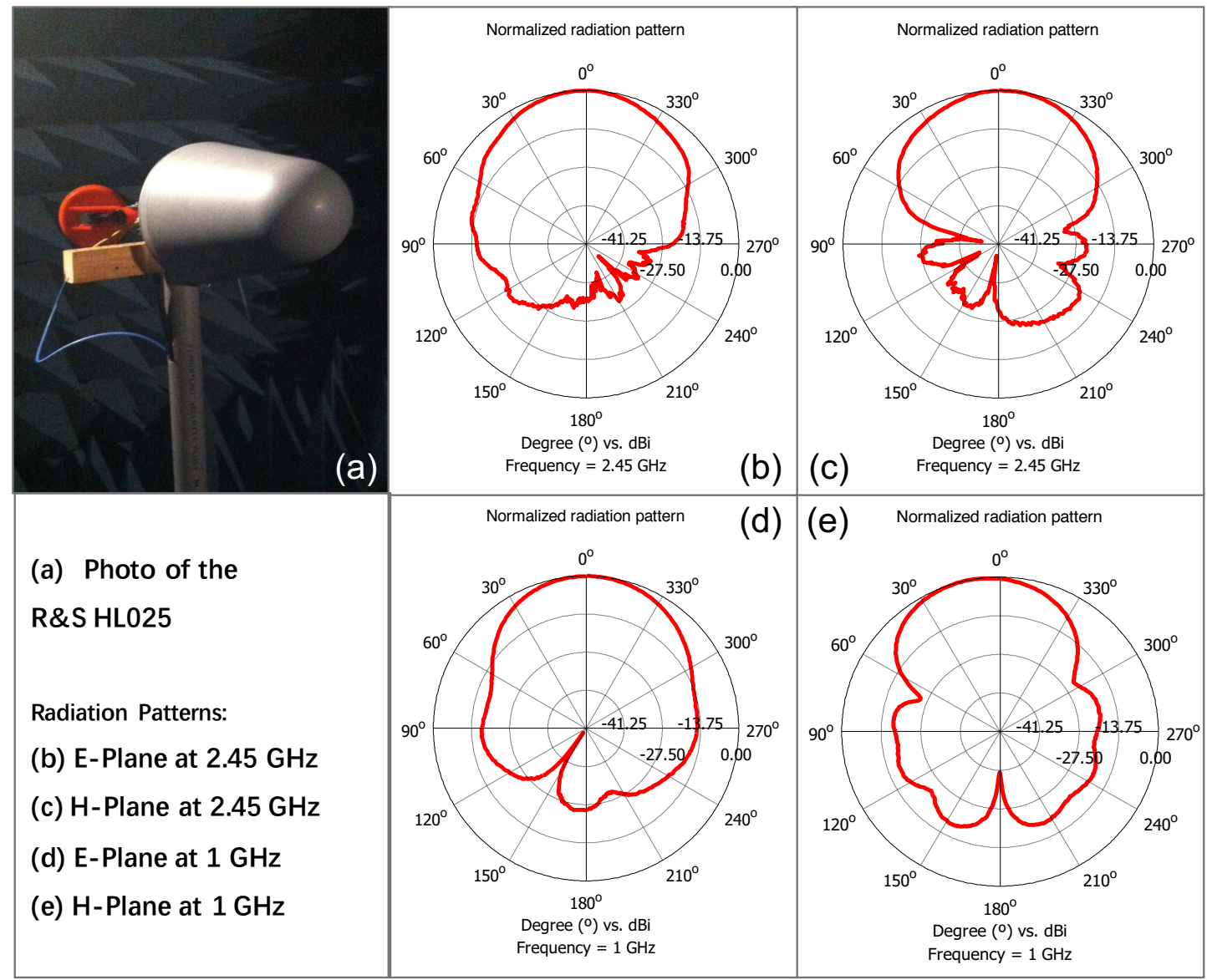

Figure 2. The photo of the R\&S HL025 log-periodic antenna and the radiation patterns at two frequencies. 


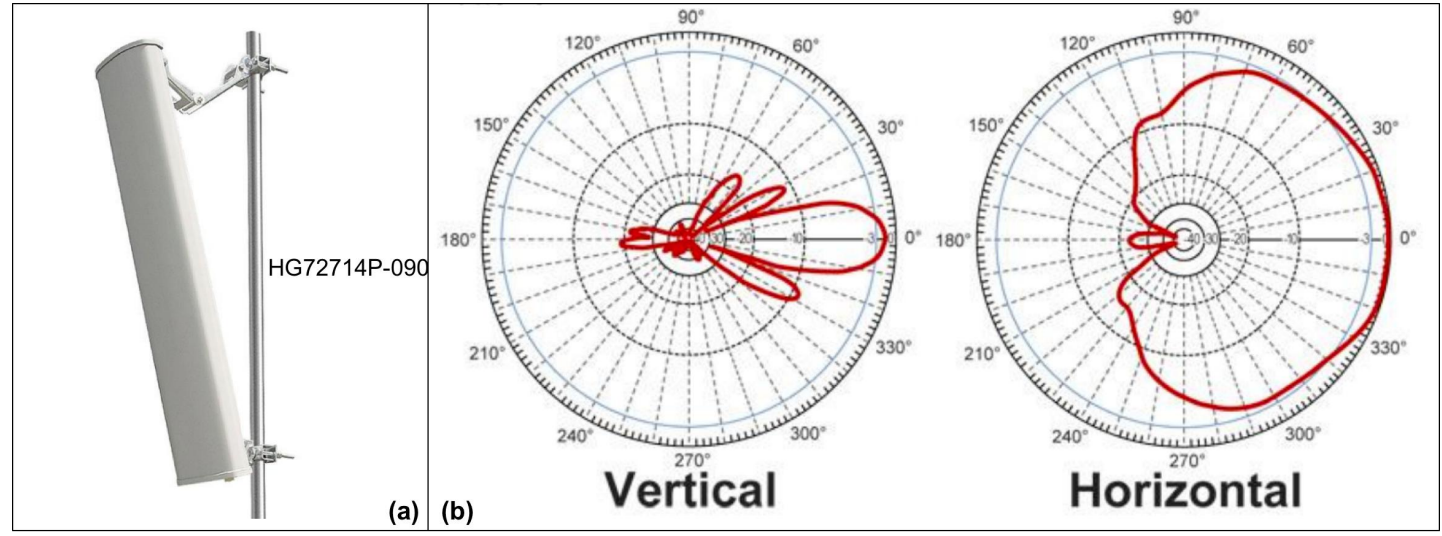

(a) HG72714P-090 panel antenna photo and radiation patterns.

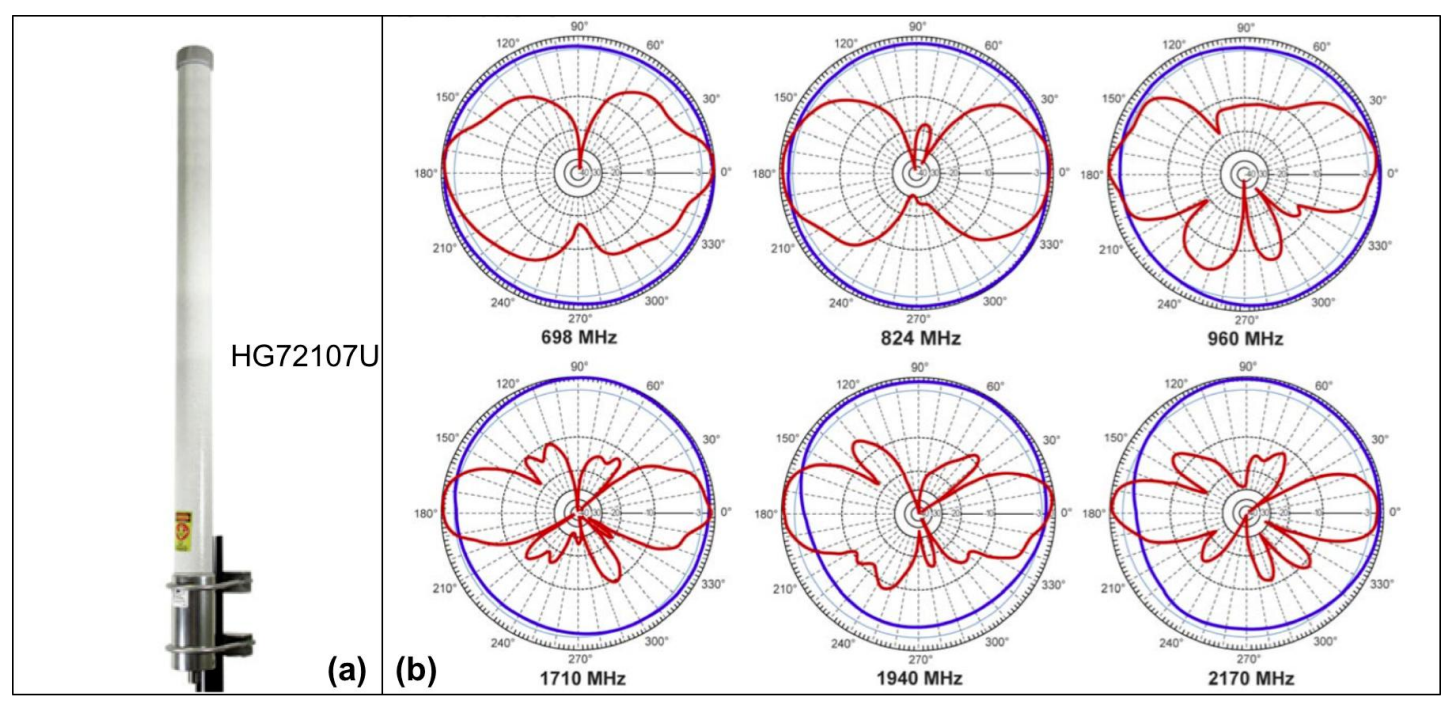

(b) HG72107U-PRO omni-directional antenna photo and radiation patterns.

Figure 3. Antennas applied in the measurements in HSR, via: www.L-com.com. 